\title{
Interim Sanitary Landfill Groundwater Monitoring Report, 1996 Annual Report
}

by

L. A. Bagwell

Westinghouse Savannah River Company

Savannah River Site

Aiken, South Carolina 29808

Exploration Rosources

This paper was prepared in connection with work done under the abovelcontract number with the U. S.

Department of Energy. By acceptance of this paper, the publisher and/or recipient acknowledges the U.S.

Government's right to retain a nonexclusive, royalty-free license in and to any copyright covering this paper, along with the right to reproduce and to authorize others to reproduce all or part of the copyrighted paper. 
WSRC-TR-96-0186-4

Unclassified

\title{
INTERIM SANITARY LANDFILL GROUNDWATER MONITORING REPORT (U)
}

\author{
1996 ANNUAL REPORT
}

Publication Date: January 1997

Key Words

barium

LFW wells

1,1,1-trichloroethane trichlorofluoromethane

zinc

Westinghouse Savannah River Company

Savannah River Site

Aiken, SC 29808 
Prepared by

\section{Environmental Protection Department \\ Environmental Geochemistry Group \\ Westinghouse Savannah River Company \\ Aiken, SC}

and

Exploration Resources, Inc.

Athens, GA 


\section{DISCLAIMER}

This report was prepared as an account of work sponsored by an agency of the United States Government. Neither the United States Government nor any agency thereof, nor any of their employees, makes any warranty, express or implied, or assumes any legal liability or -responsibility for the accuracy, completeness, or usefulness of any information, apparatus, product, or process disclosed, or represents that its use would not infringe privately owned rights. Reference herein to any specific commercial product, process, or service by trade name, trademark, manufacturer, or otherwise does not necessarily constitute or imply its endorsement, recommendation, or favoring by the United States Government or any agency thereof. The views and opinions of authors expressed herein do not necessarily state or reflect those of the United States Government or any agency thereof.

This report has been reproduced directly from the best available copy.

Available to DOE and DOE contractors from the Office of Scientific and Technical Information, P.O. Box 62, Oak Ridge, TN 37831; prices available from (615) 576-8401.

Available to the public from the National Technical Information Service, U.S. Department of Commerce, 5285 Port Royal Road, Springfield, VA 22161. 


\section{DISCLAMIER}

Portions of this document may be illegible in electronic image products. Images are produced from the best available original document. 


\section{Abstract}

Eight wells of the LFW series monitor groundwater quality in the Steed Pond Aquifer (Water Table) beneath the Interim Sanitary Landfill at the Savannah River Site (SRS). These wells are sampled semiannually to comply with the South Carolina Department of Health and Environmental Control Modified Municipal Solid Waste Permit 025500-1120 (formerly DWP-087A) and as part of the SRS Groundwater Monitoring Program.

Trichlorofluoromethane and 1,1,1-trichloroethane were elevated in one sidegradient well and one downgradient well during 1996. Zinc was elevated in three downgradient wells and also was detected in the associated laboratory blanks for two of those wells. Specific conductance was elevated in one background well and one sidegradient well. Barium and copper exceeded standards in one sidegradient well, and dichloromethane (a common laboratory contaminant) was elevated in another sidegradient well. Barium, copper, and dichloromethane were detected in the associated blanks for these wells, also.

The groundwater flow direction in the Steed Pond Aquifer (Water Table) beneath the Interim Sanitary Landfill was to the southeast (universal transverse Mercator coordinates). The flow rate in this unit was approximately $210 \mathrm{ft} /$ year during first quarter 1996 and $180 \mathrm{ft} / \mathrm{yr}$ during third quarter 1996. 
WSRC-TR-96-0186-4 Unclassified

THIS PAGE LEFT BLANK INTENTIONALLY. 


\section{Contents}

Page

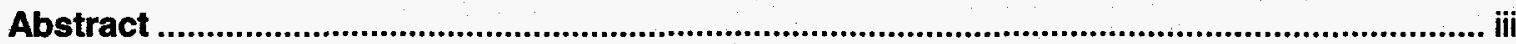

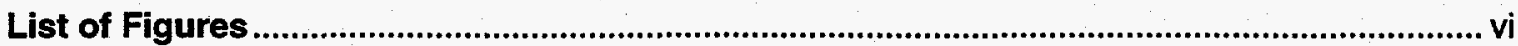

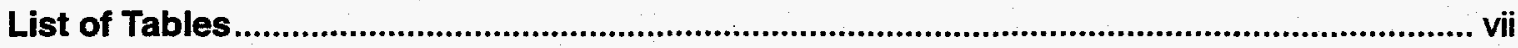

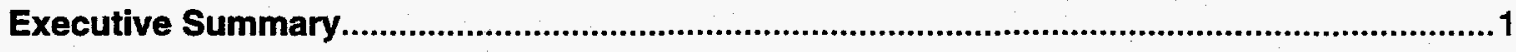

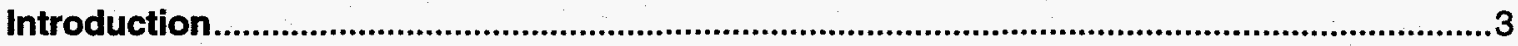

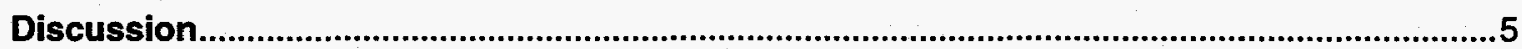

Groundwater Monitoring Data....................................................................................

Integrity of the Monitoring Well Network..........................................................................5

Analytical Results Exceeding Standards ..................................................................5

Water Levels..................................................................................................................6

Groundwater Flow Directions and Rates .......................................................................

Upgradient versus Downgradient Results ..................................................................... 7

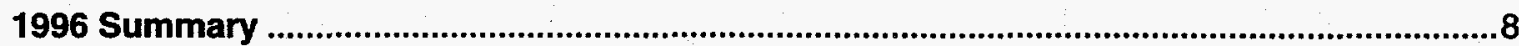

Appendix A_Standards ............................................................................................

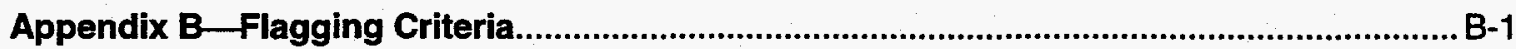

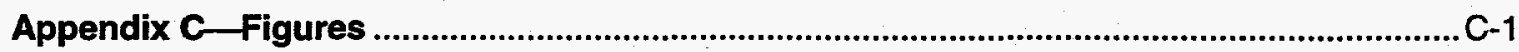

Appendix D_Groundwater Monitoring Results Tables....................................................... D-1

Appendix E-Data Quality/Usability Assessment ...............................................................

Appendix F-Groundwater Monitoring Results for Individual Wells for Special Study...... F-1 


\section{List of Figures}

Page

1. Location of the Interim Sanitary Landfill at the Savannah River Site.................................3

2. Location of Monitoring Wells at the Interim Sanitary Landfill ...........................................

3. Piezometric Surface Map of Steed Pond Aquifer (Water Table) at the Interim Sanitary Landfill, First Quarter 1996. C-5

4. Piezometric Surface Map of Steed Pond Aquifer (Water Table) at the Interim Sanitary Landfill, Third Quarter 1996 .........................................................................6

5. Regional Correlation of Hydrostratigraphic and Lithostratigraphic Nomenclature............ C-7 


\section{List of Tables}

Page

B-1. Flagging Criteria

B-3

D-1. Maximum Levels of Constituents Exceeding the Practical Quantitation Limits or Tolerance Limits

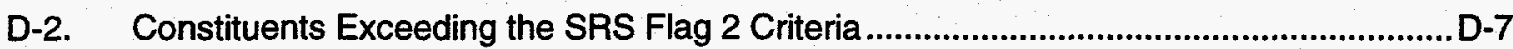

D-3. Groundwater Monitoring Results for Individual Wells D-8

F-1. Groundwater Monitoring Results for Individual Wells for the Special Study, First Quarter 1996 $F-4$

F-2 Groundwater Monitoring Results for Individual Wells for the Special Study, Second Quarter 1996 F-8 
WSRC-TR-96-0186-4

Unclassified

THIS PAGE LEFT BLANK INTENTIONALLY. 
The Sanitary Landfill at the Savannah River Site is composed of the original 32-acre landfill, plus expansion areas to the north and south that add 16 and 22 acres, respectively, to the facility. Eight LFW wells surround the area originally set aside for the Northern Expansion, now known as the Interim Sanitary Landfill. These wells monitor the groundwater quality in the Steed Pond Aquifer (Water Table) beneath the Interim Sanitary Landfill. They are sampled semiannually for selected indicator parameters, metals, and organics to comply with the South Carolina Department of Health and Environmental Control (SCDHEC) Modified Municipal Solid Waste Permit 025500-1120 (formerly DWP-087A) (SCDHEC, 1995), as designated in the Interim Sanitary Landfill Groundwater Monitoring Program (WSRC, 1994).

During first quarter 1996, 6 ( 3 downgradient, 1 background, and 2 sidegradient) of the 8 monitoring wells ( 75 percent) at the Interim Sanitary Landfill contained levels of barium, copper, specific conductance, 1,1,1-trichloroethane, trichlorofluoromethane, or zinc that exceeded the standards. Barium and copper in sidegradient well LFW 28, dichloromethane (a common laboratory contaminant) in sidegradient well LFW 32, and zinc in downgradient wells LFW 75C and $75 \mathrm{D}$ had results with $V$ modifiers, indicating that the elevated analytes also were detected in their respective associated laboratory blanks. The value for 1,1,1-trichloroethane was estimated, indicated by the $J$ modifier, because quantitation in the sample or in associated quality control samples did not meet specifications.

During third quarter 1996, 3 of the monitoring wells ( 38 percent) at the Interim Sanitary Landfill contained elevated constituents. Trichlorofluoromethane and 1,1,1-trichloroethane were elevated in well LFW 32 and downgradient well LFW 74D, dichloromethane was elevated in well LFW 32, and barium and specific conductance were elevated in well LFW 28. Dichloromethane was detected in the associated blank for well LFW 32 (modifier $V$. The value for trichlorofluoromethane in well LFW 74D was estimated because quantitation in the sample or in associated quality control samples did not meet specifications; the result may be an underestimation of the true value due to analytical bias (modifier $J 1$ ).

In addition to regularly scheduled first quarter and third quarter sampling events, several wells in the LFW series were sampled for special investigation during 1996. The results of these special sampling events are summarized in Appendix $F$.

The groundwater flow direction in the Steed Pond Aquifer (Water Table) beneath the Interim Sanitary Landfill during first and third quarters 1996 was to the southeast (universal transverse Mercator coordinates) toward Upper Three Runs Creek. The estimated flow rate in this aquifer was $210 \mathrm{ft} /$ year during first quarter 1996 and $180 \mathrm{ft} /$ year during third quarter 1996 . These directions and flow rates are similar to directions and flow rates during 1995. 
THIS PAGE LEFT BLANK INTENTIONALLY. 


\section{Introduction}

The Sanitary Landfill (Figures 1 and 2, Appendix C) is located southwest of Road C, about midway down the slope from the Aiken Plateau to Upper Three Runs Creek. The following description outlines important events in the history of the landfill:

- The original 32-acre Sanitary Landfill began receiving sanitary waste, including paper and paper products, plastics, scrap metal, rubber, food waste, glass, packing material, miscellaneous construction debris, empty paint cans, and other nonradioactive waste materials in 1974 (Heffner and Exploration Resources, 1991).

- On June 12, 1978, the South Carolina Department of Health and Environmental Control (SCDHEC) issued permit DWP-087 to the U.S. Department of Energy (DOE) for the operation of the Sanitary Landfill for inert and domestic solid waste disposal.

- On September 9, 1986, SCDHEC issued permit DWP-087A, which allowed expansion areas to the north and south of the original 32-acre landfill, adding 16 and 22 acres, respectively (WSRC, 1993). These areas were known as the Northern Expansion and the Southern Expansion. Later, the Northern Expansion was renamed the Interim Sanitary Landfill.

- The original landfill was filled to capacity in early 1987 (WSRC, 1990).

- In 1988, the Sanitary Landfill was placed on the Resource Conservation and Recovery Act (RCRA) Facility Investigation (RFI) list because elevated levels of hazardous constituents were detected in the groundwater beneath the facility (WSRC, 1990).

- A groundwater quality assessment plan for the Sanitary Landfill was submitted to SCDHEC in 1990 (WSRC, 1990).

- $\quad$ SCDHEC renewed permit DWP-087A on July 24, 1991 (SCDHEC, 1991).

- As a result of Settlement Agreement 91-51-SW, effective August 24, 1991, the Sanitary Landfill became subject to RCRA requirements because wipes and rags contaminated with RCRAlisted solvents were disposed of there.

- As a result of the RCRA permit investigation, the U.S. Environmental Protection Agency (EPA) removed the Sanitary Landfill from the RFI/Remedial Investigation unit list in August 1991.

- The Interim Sanitary Landfill (Northern Expansion) began operations in mid-1992 when the Southern Expansion was filled (WSRC, 1993).

- The Savannah River Site submitted a closure plan for the Sanitary Landfill to SCDHEC on February 28, 1993.

- A RCRA Part B post-closure care permit application was submitted to SCDHEC on March 31, 1993.

- A modification to the Sanitary Landfill groundwater quality assessment plan was approved by SCDHEC in November 1993. The modified plan included further characterization studies and proposed the installation of additional monitoring wells. Thirty-two new monitoring wells were sampled for the first time during second quarter 1994.

- The Interim Sanitary Landfill Groundwater Monitoring Program was submitted to SCDHEC in July 1994 in support of the renewal application for permit DWP-087A (WSRC, 1994).

- The Interim Sanitary Landfill, the last open portion of the Sanitary Landfill, curtailed operations in November 1994. 
- Two new well clusters were installed in the Interim Sanitary Landfill in November 1994 based on recommendations made in the groundwater monitoring program (WSRC, 1994) to provide downgradient wells for the Interim Sanitary Landfill. These wells were sampled for the first time during first quarter 1995.

- The modification to municipal solid waste permit DWP-087A was approved on February 2, 1995, and included information regarding the monitoring of the eight designated Interim Sanitary Landfill wells. The permit, which became effective on February 22, 1995, was renumbered as 025500-1120 because of SCDHEC's new landfill tracking system.

The SRS Environmental Protection Department/Environmental Monitoring Section (EPD/EMS) samples the eight monitoring wells at the Interim Sanitary Landfill semiannually for selected parameters. The Solid Waste Management Department provides semiannual reports of monitoring results to SCDHEC to meet regulatory requirements. 


\section{Discussion}

\section{Groundwater Monitoring Data}

Groundwater samples were collected from the LFW wells at the Interim Sanitary Landfill during March for first quarter 1996 and during September for third quarter 1996. In addition to the first quarter and third quarter sampling events, several wells in the LFW series were sampled for special investigation during 1996. The results of these special sampling events are summarized in Appendix F.

The sampling procedure (WSRC, 1992) requires evacuation of a minimum of two well volumes and stabilization of $\mathrm{pH}$, specific conductance, and turbidity prior to sample collection. Stability is established when a minimum of three successive measurements, taken for each stability parameter within a given time period, are within a specified tolerance range. If a well pumps dry before two well volumes are purged or before stabilization is achieved, it must be revisited within 24 hours for the data to be considered from a single sampling event. On the second visit within 24 hours, samples are taken without purging or stability measurements; thus, these samples may not be representative of groundwater quality.

Samples from LFW wells at the Interim Sanitary Landfill were analyzed for selected indicator parameters, metals, and organics. For simplicity, results that equaled or exceeded standards are described as exceeding standards, above standards, or as elevated. The following standards were used in this report:

- practical quantitation limits (PQLs) as established in the Interim Sanitary Landfill Groundwater Monitoring Program (WSRC, 1994). Bromochloromethane and cis-1,2-dichloroethylene were not assigned PQLs; therefore, no standards were established for these constituents (see Appendix A).

- tolerance limits calculated for copper, lead, pH, specific conductance, and zinc provided in the Interim Sanitary Landfill Groundwater Monitoring Program (WSRC, 1994) (see Appendix A)

- SRS flagging criteria based on Primary Drinking Water Standards, Secondary Drinking Water Standards, or method detection limits (see Appendix B)

\section{Integrity of the Monitoring Well Network}

Wells LFW 28, 32, 43C, 43D, 74C, 74D, 75C, and 75D presently monitor groundwater quality in the Steed Pond Aquifer (Water Table) beneath the Interim Sanitary Landfill (Figure 2, Appendix C). The designated background wells at the Interim Sanitary Landfill are wells LFW 43C and 43D.

SRS rehabilitates or replaces wells that do not produce representative groundwater samples. A complete record of well installations, replacements, and abandonments at the Sanitary Landfill is provided in the EPD/EMS well inventory (EPD/EMS, 1996). During 1996, no Interim Sanitary Landfill wells were abandoned, and no new wells were installed.

Table D-3 (Appendix D) lists the number of well volumes purged from each of the LFW wells during first and third quarters 1996 and provides sampling codes $(I, P, X$, etc. [see Appendix $D$ front matter]) that describe unusual sampling events. Well LFW 28 went dry during purging (sampling code $X$ ) during first quarter 1996.

\section{Analytical Results Exceeding Standards}

Results for analytes that exceeded standards (Appendix A) during first or third quarters 1996 are summarized in Table D-1 (Appendix D) and described in the following text: 
During first quarter 1996, 6 of the 8 LFW wells at the Interim Sanitary Landfill contained elevated constituents.

- Barium, copper, and specific conductance were elevated in well LFW 28 with values of $31 \mu \mathrm{g} / \mathrm{L}, 37 \mu \mathrm{g} / \mathrm{L}$, and $50 \mu \mathrm{S} / \mathrm{cm}$, respectively. These results may be due to high turbidity ( 8 NTU) in the well. Barium and copper also were detected in the associated laboratory blank.

- 1,1,1-Trichloroethane and trichlorofluoromethane were elevated in well LFW 32 with values of $7.9 \mu \mathrm{g} / \mathrm{L}$ and $96 \mu \mathrm{g} / \mathrm{L}$, respectively.

- Trichlorofluoromethane and zinc were elevated in well LFW 74D with values of $15 \mu \mathrm{g} / \mathrm{L}$ and $54 \mu \mathrm{g} / \mathrm{L}$, respectively.

- Specific conductance was elevated in well LFW $43 C$ at $170 \mu \mathrm{S} / \mathrm{cm}$.

- Zinc was elevated in wells LFW $75 \mathrm{C}$ and 75D with values of $32 \mu \mathrm{g} / \mathrm{L}$ and $43 \mu \mathrm{g} / \mathrm{L}$, respectively, and was detected in the associated laboratory blanks as well.

During third quarter 1996, 3 of the 8 LFW wells contained elevated constituents.

- Trichlorofluoromethane was elevated in wells LFW 32 and 74D at $170 \mu \mathrm{g} / \mathrm{L}$ and $39 \mu \mathrm{g} / \mathrm{L}$, respectively.

- 1,1,1-Trichloroethane was elevated in wells LFW 32 and $74 D$ with values of $13 \mu \mathrm{g} / \mathrm{L}$ and $6.4 \mu \mathrm{g} / \mathrm{L}$, respectively.

- Barium and specific conductance were elevated in well LFW 28 with values of $26 \mu \mathrm{g} / \mathrm{L}$ and $1.9 \mu \mathrm{S} / \mathrm{cm}$, respectively.

- Dichloromethane was elevated in well LFW 32 at $37 \mu \mathrm{g} / \mathrm{L}$ and also was detected in the associated laboratory blank.

Results for analytes that exceeded SRS Flag 2 criteria (Appendix B) during first or third quarters 1996 are summarized in Table D-2 (Appendix D).

Table D-3 (Appendix D) shows the results during first and third quarters 1996 for all of the constituents and indicates the analytical laboratories that conducted the analyses, the dilution factors used in the analyses, and the analyses that received modifiers (which help define laboratory accuracy and precision) or that exceeded the EPA-approved holding times. Constituent results in Table D-3 that appear to equal the final standards but are not marked in the ST column (exceeded standard) are below the standard in the database. Database results, the results that are compared to the standards, contain more significant digits than the results given in this report. Apparent discrepancies are due to the rounding of reported results.

In addition to the results tables, Appendix $D$ provides definitions of the abbreviations and modifiers used in the results tables as well as descriptions of holding times, data rounding, and data qualification practices. Appendix $E$ provides a general assessment of the quality and usability of EPD/EMS data.

\section{Water Levels}

Average Water Elevations at the Interim Sanitary Landfill

\begin{tabular}{|lll|}
\hline & 1995 Average & 1996 Average \\
Quarter & Water Elevation $(\mathrm{ft} \mathrm{msl})$ & Water Elevation $(\mathrm{ft} \mathrm{msl})$ \\
\hline First & 164.6 & 163.4 \\
Third & 163.4 & 167.8 \\
\hline
\end{tabular}




\section{Groundwater Flow Directions and Rates}

Historically, the groundwater flow direction in the Steed Pond Aquifer (Water Table) hydrostratigraphic unit beneath the Sanitary Landfill has been to the southeast (universal transverse Mercator [UTM] coordinates) toward Upper Three Runs Creek (WSRC, 1995). During first and third quarters 1996, the flow direction at the Interim Sanitary Landfill was to the southeast (Figures 3 and 4, Appendix C). Figure 5 presents the regional correlation of hydrostratigraphic and lithostratigraphic nomenclature.

Horizontal flow rate calculations provide estimates of the transport rate of constituents originating from the Interim Sanitary Landfill. Flow rates are estimated using the following equation:

$$
\text { Flow }\left(\mathrm{ft} / \text { day) }=\frac{\text { Hydraulic Conductivity (ft/day) }}{\text { Porosity (unitless) }} \times \frac{d h(\mathrm{ft})}{d /(\mathrm{ft})}\right.
$$

where the hydraulic conductivity constant is $16 \mathrm{ft} /$ day (WSRC, 1994), the effective porosity value is 20 percent, the change in head is $d h$, and the horizontal distance along each flow direction arrow is dl.

Flow path length is calculated to the nearest $10 \mathrm{ft}$. Flow rate per day is calculated to two significant figures, then multiplied by 365 and rounded to two significant figures for the flow rate per year. Flow rate estimates vary depending upon the vertical gradient between wells, the size of the area under consideration, the number of data points, and the length and location of the flow path. Because these are inferred or estimated parameters, flow rate estimates should be considered accurate to an order of magnitude only.

The approximate groundwater flow rate in the Steed Pond Aquifer (Water Table) during first quarter 1996 was estimated as follows (Figure 3, Appendix C):

$$
\frac{16}{0.20} \times \frac{6}{840} \approx 0.57 \mathrm{ft} / \mathrm{day}
$$

$0.57 \mathrm{ft} /$ day $\times 365$ days $\approx 210 \mathrm{ft} /$ year

The approximate groundwater flow rate in the Steed Pond Aquifer (Water Table) during third quarter 1996 is estimated as follows (Figure 4, Appendix C):

$$
\frac{16}{0.20} \times \frac{6}{960} \approx 0.5 \mathrm{ft} / \mathrm{day}
$$

$0.5 \mathrm{ft} /$ day $\times 365$ days $\approx 180 \mathrm{ft} /$ year

\section{Upgradient versus Downgradient Results}

Wells LFW 43C and 43D in the Interim Sanitary Landfill are background wells for the Steed Pond Aquifer (Water Table). Wells LFW 74C, 74D, 75C, and 75D are downgradient, and the remaining wells are sidegradient (Figures 2, 3, and 4, Appendix C).

Specific conductance was elevated in background well LFW 43C during first quarter 1996. Downgradient well LFW 74D contained elevated levels of 1,1,1-trichloroethane during third quarter 1996 and elevated levels of trichlorofluoromethane during first and third quarters 1996. Zinc was elevated in downgradient wells LFW 74D, 75C, and 75D during first quarter 1996. 
During first quarter 1996, 6 of the 8 LFW wells ( 3 downgradient, 1 background, and 2 sidegradient) at the Interim Sanitary Landfill contained levels of barium, copper, specific conductance, 1,1,1-trichloroethane, trichlorofluoromethane, or zinc that exceeded the standards. Barium and copper in well LFW 28, dichloromethane (a common laboratory contaminant) in well LFW 32, and zinc in wells LFW 75C and 75D had results with $V$ modifiers, indicating that the elevated analytes also were detected in their respective associated laboratory blanks. The value for 1,1,1-trichloroethane was estimated, indicated by the $J$ modifier, because quantitation in the sample or in associated quality control samples did not meet specifications.

During third quarter 1996, 3 of the LFW wells at the Interim Sanitary Landfill contained elevated constituents. Trichlorofluoromethane and 1,1,1-trichloroethane were elevated in one downgradient well and one sidegradient well, dichloromethane was elevated in one sidegradient well, and barium and specific conductance were elevated in one sidegradient well. Dichloromethane was detected in the associated blank for well LFW 32 (modifier $\eta$. The value for trichlorofluoromethane in well LFW 74D was estimated because quantitation in the sample or in associated quality control samples did not meet specifications; the result may be an underestimation of the true value due to analytical bias (modifier J1).

In addition to regularly scheduled first quarter and third quarter sampling events, several wells in the LFW series were sampled for special investigation during 1996. The results of these special sampling events are summarized in Appendix F.

The groundwater flow direction in the Steed Pond Aquifer (Water Table) beneath the Interim Sanitary Landfill is to the southeast (UTM coordinates) toward Upper Three Runs Creek. The flow rate in this aquifer was estimated at approximately $210 \mathrm{ft} / \mathrm{yr}$ during first quarter 1996 and at approximately $180 \mathrm{ft} /$ year during third quarter 1996. Flow directions and rates during 1996 were similar to 1995 findings. 
WSRC-TR-96-0186-4

Unclassified

\section{Appendix A}

\section{Standards}


THIS PAGE LEFT BLANK INTENTIONALLY. 


\section{Standards}

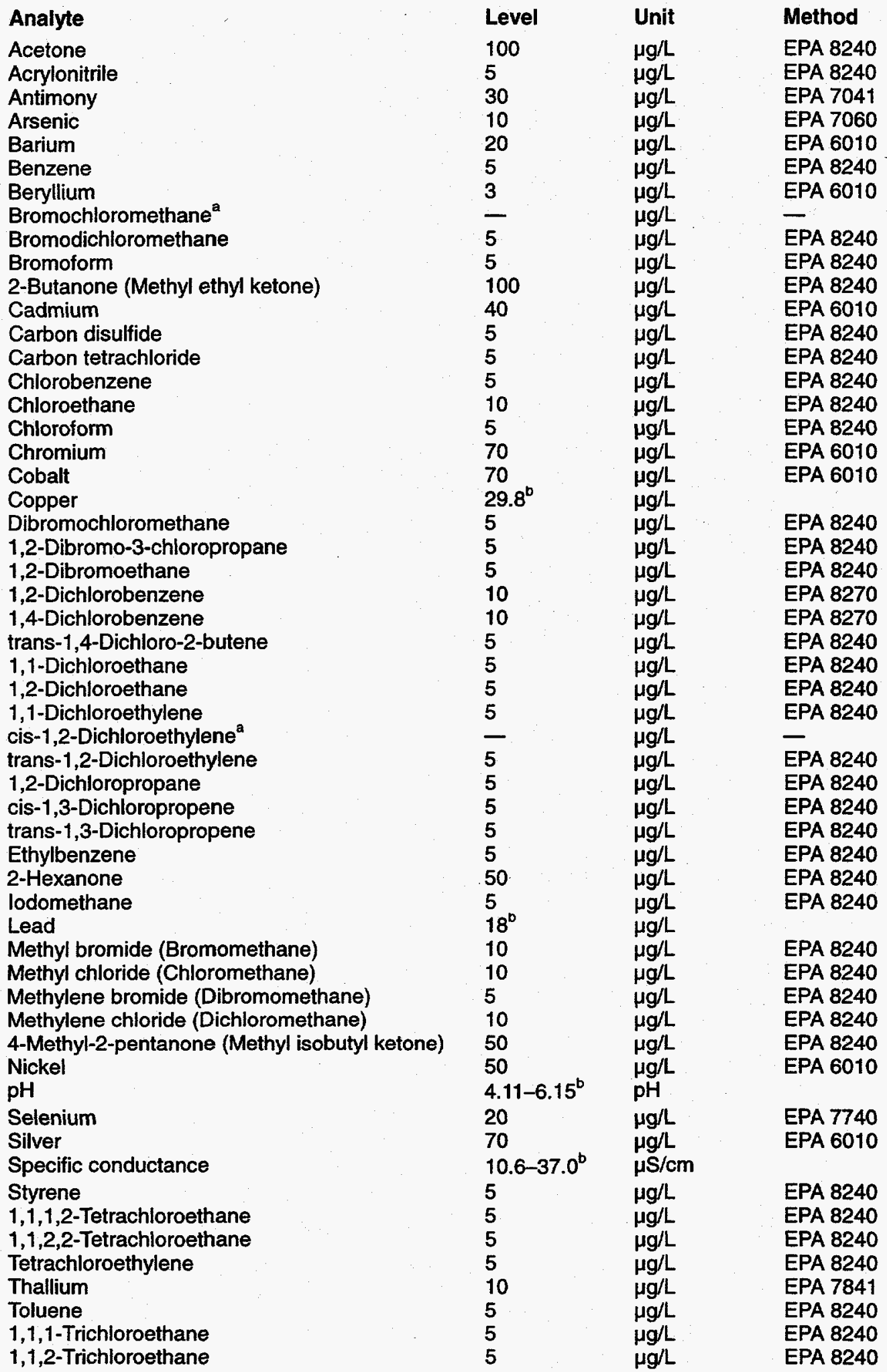


Analyte

Trichloroethylene

Trichlorofluoromethane

1,2,3-Trichloropropane

Vanadium

Vinyl acetate

Vinyl chloride (Chloroethene)

Xylene

Zinc

$\begin{array}{ll}\text { Level } & \text { Unit } \\ 5 & \mu g / L \\ 5 & \mu g / L \\ 5 & \mu g / L \\ 80 & \mu g / L \\ 5 & \mu g / L \\ 10 & \mu g / L \\ 5 & \mu g / L \\ 29.3^{\circ} & \mu g / L\end{array}$

Method

EPA 8240

EPA 8240

EPA 8240

EPA 6010

EPA 8240

EPA 8240

EPA 8240

a No PQL is available for this constituent.

b Tolerance limits for copper, lead, $\mathrm{pH}$, specific conductance, and zinc are set according to the Interim Sanitary Landfill Groundwater Monitoring Program, Table 2-4. 
WSRC-TR-96-0186-4

Unclassified

\section{Appendix B}

\section{Flagging Criteria}


THIS PAGE LEFT BLANK INTENTIONALLY. 


\section{Flagging Criteria}

Beginning first quarter 1992, flagging criteria were established for all constituents analyzed as part of the EPD/EMS Groundwater Monitoring Program. Only the flagging criteria for environmental screening analyses are used to trigger scheduling for designated welis in the environmental screening program, while all flagging criteria in Table B-1 are used to flag constituents in the analytical results tables.

The following rules are used in determining flagging criteria and scheduling sampling:

- Flag 0: Analysis for a constituent below Flag 1 is classified as Flag 0 . Constituents classified as Flag 0 in each well series will be scheduled for analyses only by custodian request or as part of the triennial environmental screening program for wells designated as environmental screening wells.

- Flag 1: The Flag 1 criterion for a constituent equals half of the EPA primary drinking water standard (PDWS), the EPA proposed PDWS, or the EPA secondary drinking water standard (SDWS) for that constituent. If a constituent does not have an EPA drinking water standard, the Flag 1 criterion equals 5 times a recently published 90 th percentile detection limit obtained by one of the primary laboratories. If a constituent exceeds Flag 1 in a monitoring well series, environmental screening wells in that series are sampled and analyzed for that constituent once a year. If a constituent falls below Flag 1 for three consecutive sampling episodes, the well's flag is reduced from Flag 1 status to Flag 0 status and follows the rules for Flag 0.

- Flag 2: The Flag 2 criterion for a constituent equals the EPA PDWS, the EPA proposed PDWS, or the EPA SDWS for that constituent. If a constituent does not have a drinking water standard, the Flag 2 criterion equals 10 times a recently published 90th percentile detection limit obtained by one of the primary laboratories. If a constituent exceeds Flag 2 in a monitoring well series, environmental screening wells in that series are sampled and analyzed for that constituent once a year. If a constituent falls below Flag 2 for three consecutive sampling episodes, the well's flag is reduced from Flag 2 status to Flag 1 or Flag 0 status and follows the rules for the lower flag.

The following constituents are exceptions to the flagging rules:

- Specific conductance and $\mathrm{pH}$, which are indicator parameters, have flagging criteria but do not trigger the scheduling mechanism. No flags are set for alkalinity, 5-day biochemical oxygen demand, calcium, carbonate, chemical oxygen demand, magnesium, potassium, silica, sodium, total dissolved solids, total phosphates (as P), and total phosphorous because they are indicator parameters or major cations. Analyses for these constituents are conducted as part of the triennial environmental screening program or by special request.

- Aesthetic analyses such as color, odor, corrosivity, Eh, turbidity, and surfactants are not assigned flagging criteria but are analyzed by special request.

- Common laboratory contaminants and cleaners including phthalates, dichloromethane, ketones, and toluene are not assigned flagging criteria unless they have PDWS. These constituents are analyzed by special request.

Table B-1. Flagging Criteria

Analyte

Acenaphthene

Acenaphthylene

Acetone

Acetonitrile (Methyl cyanide)

Acetophenone

$\begin{array}{lll}\text { Unit } & \text { Flag 1 } & \text { Flag 2 } \\ \mu \mathrm{g} / \mathrm{L} & 5.1 & 10.2 \\ \mu \mathrm{g} / \mathrm{L} & 5.1 & 10.2 \\ \mu \mathrm{g} / \mathrm{L} & 500 & 1,000 \\ \mu \mathrm{g} / \mathrm{L} & 50 & 100 \\ \mu \mathrm{g} / \mathrm{L} & 85 & 170\end{array}$

\author{
Source \\ EPA Method 8270 \\ EPA Method 8270 \\ EPA Method 8240 (Set by EPD/EMS) \\ EPA Method 8240 \\ EPA Method 8270
}




Analyte
2-Acetylaminofluorene
Acrolein
Acrylonitrile
Actinium-228
Alachlor
Aldicarb
Aldicarb sulfone
Aldicarb sulfoxide
Aldrin
Alkalinity (as CaCO ${ }_{3}$ )
Allyl chloride
Aluminum
Aluminum, dissolved
Aluminum, total recoverable
Americium-241
Americium-243
4-Aminobiphenyl
Ammonia
Ammonia nitrogen
Aniline
Anthracene
Antimony
Antimony, dissolved
Antimony, total recoverable
Antimony-124
Antimony-125
Aramite
Arsenic
Arsenic, dissolved
Arsenic, total recoverable
Asbestos
Atrazine
Azobenzene
Barium
Barium, dissolved
Barium, total recoverable
Barium-133
Barium-140
Benzene
alpha-Benzene hexachloride
beta-Benzene hexachloride
delta-Benzene hexachloride
Benzidine
Benzo[a]anthracene
Benzo[b]fluoranthene
Benzo[kfluoranthene
Benzoic acid
Benzo[g, h,jperylene
Benzo[a]pyrene
1,4-Benzoquinone
Benzyl alcohol
Beryllium

Interim Sanitary Landfill

\begin{tabular}{|c|c|c|c|}
\hline Unit & Flag 1 & Flag 2 & Source \\
\hline$\mu g / L$ & 81 & 162 & EPA Method 8270 \\
\hline$\mu g / L$ & 166.5 & 333 & EPA Method 8240 \\
\hline$\mu g / L$ & 250 & 500 & EPA Method 8240 \\
\hline$\mu \mathrm{Ci} / \mathrm{mL}$ & $1.64 \mathrm{E}-06$ & $3.27 E--06$ & Proposed DWS (EPA, 1991a) \\
\hline$\mu g / L$ & 1 & 2 & Final DWS (CFR, 1995a) \\
\hline$\mu \mathrm{g} / \mathrm{L}$ & 1.5 & 3 & Final DWS (CFR, 1995a) \\
\hline$\mu g / L$ & 1 & 2 & Final DWS (CFR, 1995a) \\
\hline$\mu \mathrm{g} / \mathrm{L}$ & 2 & 4 & Final DWS (CFR, 1995a) \\
\hline$\mu g / L$ & 0.4 & 0.8 & EPA Method 8080 \\
\hline$\mu g / L$ & $\begin{array}{l}\text { No flag } \\
416.5\end{array}$ & $\begin{array}{l}\text { No flag } \\
833\end{array}$ & $\begin{array}{l}\text { Set by EPD/EMS } \\
\text { EPA Method } 8240\end{array}$ \\
\hline$\mu g / L$ & 25 & 50 & Secondary DWS (CFR, 1995b) \\
\hline$\mu g / L$ & 25 & 50 & Secondary DWS (CFR, 1995b) \\
\hline$\mu \mathrm{g} / \mathrm{L}$ & 25 & 50 & Secondary DWS (CFR, 1995b) \\
\hline$\mu \mathrm{Ci} / \mathrm{mL}$ & 3.17E-09 & $6.34 \mathrm{E}-09$ & Proposed DWS (EPA, 1991a) \\
\hline$\mu \mathrm{Ci} / \mathrm{mL}$ & $3.19 E-09$ & 6.37E-09 & Proposed DWS (EPA, 1991a) \\
\hline$\mu g / L$ & 81 & 162 & EPA Method 8270 \\
\hline$\mu g / L$ & 250 & 500 & APHA Method 417B \\
\hline$\mu \mathrm{g} / \mathrm{L}$ & 500 & 1,000 & EPA Method 350.1 \\
\hline$\mu g / L$ & 81 & 162 & EPA Method 8270 \\
\hline$\mu \mathrm{g} / \mathrm{L}$ & 5.1 & 10.2 & EPA Method 8270 \\
\hline$\mu \mathrm{g} / \mathrm{L}$ & 3 & 6 & Final DWS (CFR, 1995a) \\
\hline$\mu g / L$ & 3 & 6 & Final DWS (CFR, 1995a) \\
\hline$\mu g / L$ & 3 & 6 & Final DWS (CFR, 1995a) \\
\hline$\mu \mathrm{Ci} / \mathrm{mL}$ & $3 E-08$ & $6 E-08$ & Interim Final DWS (EPA, 1977) \\
\hline$\mu \mathrm{Ci} / \mathrm{mL}$ & $1.5 E-07$ & $3 E-07$ & Interim Final DWS (EPA, 1977) \\
\hline$\mu \mathrm{g} / \mathrm{L}$ & 81 & 162 & EPA Method 8270 \\
\hline$\mu g / L$ & 25 & 50 & Final DWS (CFR, 1995a) \\
\hline$\mu g / L$ & 25 & 50 & Final DWS (CFR, 1995a) \\
\hline$\mu g / L$ & 25 & 50 & Final DWS (CFR, 1995a) \\
\hline $\begin{array}{l}\text { Fibers/L } \\
\mu g / L\end{array}$ & $\begin{array}{l}3,500,000 \\
1.5\end{array}$ & $\begin{array}{l}7,000,000 \\
3\end{array}$ & $\begin{array}{l}\text { Final DWS (CFR, 1995a) } \\
\text { Final DWS (CFR, 1995a) }\end{array}$ \\
\hline$\mu g / L$ & 50 & 100 & EPA Method 625 \\
\hline$\mu g / L$ & 1000 & 2,000 & Final DWS (CFR, 1995a) \\
\hline$\mu g / L$ & 1000 & 2,000 & Final DWS (CFR, 1995a) \\
\hline$\mu g / L$ & 1000 & 2,000 & Final DWS (CFR, 1995a) \\
\hline$\mu \mathrm{Ci} / \mathrm{mL}$ & $7.6 E-07$ & $1.52 \mathrm{E}-06$ & Proposed DWS (EPA, 1991a) \\
\hline$\mu \mathrm{Ci} / \mathrm{mL}$ & $4.5 E-08$ & $9 E-08$ & Interim Final DWS (EPA, 1977) \\
\hline$\mu g / L$ & 2.5 & 5 & Final DWS (CFR, 1995a) \\
\hline$\mu g / L$ & 0.15 & 0.3 & EPA Method 8080 \\
\hline$\mu g / L$ & 0.25 & 0.5 & EPA Method 8080 \\
\hline$\mu \mathrm{g} / \mathrm{L}$ & 0.25 & 0.5 & EPA Method 8080 \\
\hline$\mu g / L$ & 83.5 & 167 & EPA Method 8270 \\
\hline$\mu g / L$ & 0.05 & 0.1 & Proposed DWS (EPA, 1990) \\
\hline$\mu g / L$ & 0.1 & 0.2 & Proposed DWS (EPA, 1990) \\
\hline$\mu g / L$ & 0.1 & 0.2 & Proposed DWS (EPA, 1990) \\
\hline$\mu g / L$ & 5 & 10 & EPA Method 8270 \\
\hline$\mu \mathrm{g} / \mathrm{L}$ & 5.1 & 10.2 & EPA Method 8270 \\
\hline$\mu g / L$ & 0.1 & 0.2 & Final DWS (CFR, 1995a) \\
\hline$\mu \mathrm{g} / \mathrm{L}$ & 50 & 100 & EPA Method 8270 \\
\hline$\mu g / L$ & 5 & 10 & EPA Method 8270 \\
\hline$\mu g / L$ & 2 & 4 & Final DWS (CFR, 1995a) \\
\hline
\end{tabular}


Analyte

Beryllium, dissolved

Beryllium, total recoverable

Beryllium-7

5-day Biochemical oxygen demand

Bis(2-chloroethoxy) methane

Bis(2-chloroethyl) ether

Bis(2-chloroisopropyl) ether

Bis(chloromethyl) ether

Bis(2-ethylhexyl) phthalate

Bismuth-214

Boron

Boron, dissolved

Boron, total recoverable

Bromide

Bromobenzene

Bromochloromethane

Bromodichloromethane

Bromoform

Bromomethane (Methyl bromide)

4-Bromophenyl phenyl ether

n-Butylbenzene

sec-Butylbenzene

tert-Butylbenzene

Butylbenzyl phthalate

2-sec-Butyl-4,6-dinitrophenol

Cadmium

Cadmium, dissolved

Cadmium, total recoverable

Calcium

Calcium, dissolved

Calcium, total recoverable

Carbofuran

Carbon disulfide

Carbon tetrachloride

Carbon-14

Carbonate

Cerium-141 ${ }^{\mathrm{a}}$

Cerium-144

Cesium-134 ${ }^{\circ}$

Cesium-137

Chemical oxygen demand

Chlordane

alpha-Chlordane

gamma-Chlordane

Chloride

4-Chloroaniline

Chlorobenzene

Chlorobenzilate

4-Chloro-m-cresol

Chloroethane

Chloroethene (Vinyl chloride)

Chloroethyl vinyl ether

\begin{tabular}{|c|c|c|}
\hline Unit & Flag 1 & Flag 2 \\
\hline$\mu g / L$ & 2 & 4 \\
\hline$\mu g / L$ & 2 & 4 \\
\hline$\mu \mathrm{Ci} / \mathrm{mL}$ & $3 E-06$ & $6 E-06$ \\
\hline$\mu g / L$ & $\begin{array}{l}\text { No flag } \\
5.1\end{array}$ & $\begin{array}{l}\text { No flag } \\
10.2\end{array}$ \\
\hline$\mu g / L$ & 5.1 & 10.2 \\
\hline$\mu g / L$ & 100 & 200 \\
\hline$\mu \mathrm{g} / \mathrm{L}$ & 50 & 100 \\
\hline$\mu g / L$ & 3 & 6 \\
\hline$\mu \mathrm{Ci} / \mathrm{mL}$ & $9.4 E-06$ & $1.89 \mathrm{E}-05$ \\
\hline$\mu g / L$ & 2,500 & 5,000 \\
\hline$\mu \mathrm{g} / \mathrm{L}$ & 2,500 & 5,000 \\
\hline$\mu g / L$ & 2,500 & 5,000 \\
\hline$\mu g / L$ & 5,000 & 10,000 \\
\hline$\mu \mathrm{g} / \mathrm{L}$ & 25 & 50 \\
\hline$\mu \mathrm{g} / \mathrm{L}$ & 5 & 10 \\
\hline$\mu \mathrm{g} / \mathrm{L}$ & 50 & 100 \\
\hline$\mu g / L$ & 50 & 100 \\
\hline$\mu \mathrm{g} / \mathrm{L}$ & 10 & 20 \\
\hline$\mu \mathrm{g} / \mathrm{L}$ & 5.1 & 10.2 \\
\hline$\mu \mathrm{g} / \mathrm{L}$ & 5 & 10 \\
\hline$\mu g / L$ & 5 & 10 \\
\hline$\mu g / L$ & 5 & 10 \\
\hline$\mu \mathrm{g} / \mathrm{L}$ & $\begin{array}{l}\text { No flag } \\
3.5\end{array}$ & $\begin{array}{l}\text { No flag } \\
7\end{array}$ \\
\hline$\mu \mathrm{g} / \mathrm{L}$ & 2.5 & 5 \\
\hline$\mu \mathrm{g} / \mathrm{L}$ & 2.5 & 5 \\
\hline$\mu g / L$ & 2.5 & 5 \\
\hline$\mu \mathrm{g} / \mathrm{L}$ & $\begin{array}{l}\text { No flag } \\
\text { No flag } \\
\text { No flag } \\
20\end{array}$ & $\begin{array}{l}\text { No flag } \\
\text { No flag } \\
\text { No flag } \\
40\end{array}$ \\
\hline$\mu \mathrm{g} / \mathrm{L}$ & 25 & 50 \\
\hline$\mu \mathrm{g} / \mathrm{L}$ & 2.5 & 5 \\
\hline$\mu \mathrm{Ci} / \mathrm{mL}$ & $1 E-06$ & $2 E-06$ \\
\hline$\mu \mathrm{Ci} / \mathrm{mL}$ & $\begin{array}{l}\text { No flag } \\
1.5 \mathrm{E}-07\end{array}$ & $\begin{array}{l}\text { No flag } \\
\text { 3E-07 }\end{array}$ \\
\hline$\mu \mathrm{Ci} / \mathrm{mL}$ & $1.31 \mathrm{E}-07$ & $2.61 E-07$ \\
\hline$\mu \mathrm{Ci} / \mathrm{mL}$ & $4.07 E-08$ & $8.13 E-08$ \\
\hline$\mu \mathrm{Ci} / \mathrm{mL}$ & $\begin{array}{l}1 E-07 \\
\text { No flag }\end{array}$ & $\begin{array}{l}2 E-07 \\
\text { No flag }\end{array}$ \\
\hline$\mu \mathrm{g} / \mathrm{L}$ & 1 & \\
\hline$\mu \mathrm{g} / \mathrm{L}$ & 0.25 & 0.5 \\
\hline$\mu \mathrm{g} / \mathrm{L}$ & 0.25 & 0.5 \\
\hline$\mu g^{\prime} L$ & 125,000 & 250,000 \\
\hline$\mu \mathrm{g} / \mathrm{L}$ & 5 & 10 \\
\hline$\mu \mathrm{g} / \mathrm{L}$ & 50 & 100 \\
\hline$\mu \mathrm{g} / \mathrm{L}$ & 81 & 162 \\
\hline$\mu \mathrm{g} / \mathrm{L}$ & 5.1 & 10.2 \\
\hline$\mu \mathrm{g} / \mathrm{L}$ & 10 & 20 \\
\hline$\mu g / L$ & 1 & 2 \\
\hline$\mu g / L$ & 5 & 10 \\
\hline
\end{tabular}

Source

Final DWS (CFR, 1995a)

Final DWS (CFR, 1995a)

Interim Final DWS (EPA, 1977)

Set by EPD/EMS

EPA Method 8270

EPA Method 8270

EPA Method 8270

EPA Method 8270

Final DWS (CFR, 1995a)

Proposed DWS (EPA, 1991a)

EPA Method 6010

EPA Method 6010

EPA Method 6010

EPA Method 300.0

EPA Method 8260

EPA Method 8260

Final DWS (CFR, 1995a)

Final DWS (CFR, 1995a)

EPA Method 8240

EPA Method 8270

EPA Method 8260

EPA Method 8260

EPA Method 8260

Set by EPD/EMS

Final DWS (CFR, 1995a)

Final DWS (CFR, 1995a)

Final DWS (CFR, 1995a)

Final DWS (CFR, 1995a)

Set by EPD/EMS

Set by EPD/EMS

Set by EPD/EMS

Final DWS (CFR, 1995a)

EPA Method 8240

Final DWS (CFR, 1995a)

Interim Final DWS (EPA, 1977)

Set by EPD/EMS

Interim Final DWS (EPA, 1977)

Proposed DWS (EPA, 1991a)

Proposed DWS (EPA, 1991a)

Interim Final DWS (EPA, 1977)

Set by EPD/EMS

Final DWS (CFR, 1995a)

EPA Method 8080

EPA Method 8080

Secondary DWS (CFR, 1995b)

EPA Method 8270

Final DWS (CFR, 1995a)

EPA Method 8270

EPA Method 8270

EPA Method 8240

Final DWS (CFR, 1995a)

EPA Method 8240 


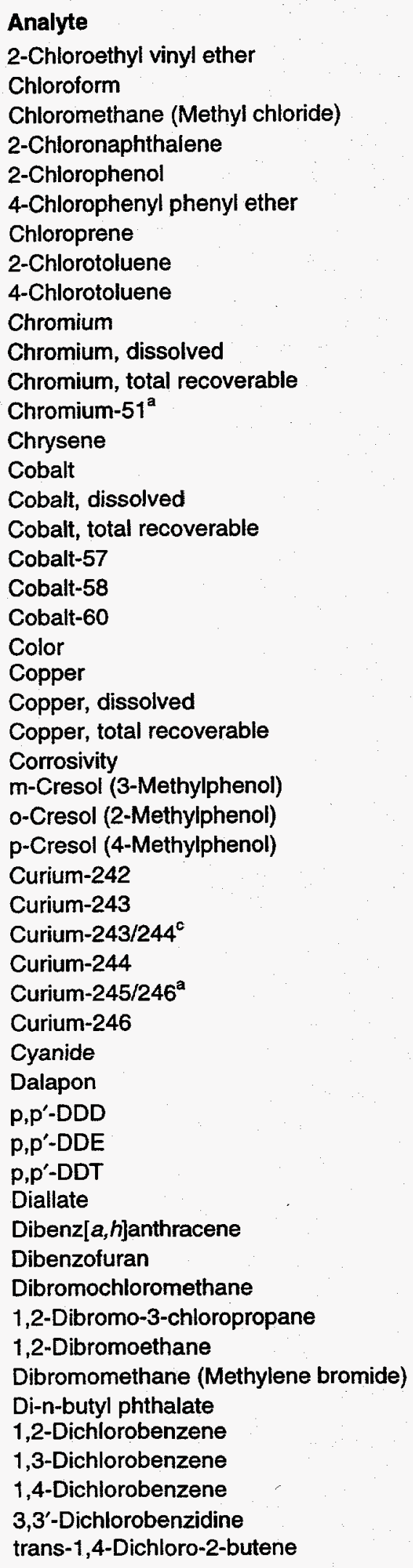

\begin{tabular}{|c|c|c|c|}
\hline Unit & Flag 1 & Flag 2 & Source \\
\hline$\mu g / L$ & 50 & 100 & EPA Method 8240 \\
\hline$\dot{\mu} g / L$ & 50 & 100 & Final DWS (CFR, 1995a) \\
\hline$\mu g / L$ & 10 & 20 & EPA Method 8240 \\
\hline$\mu g / L$ & 5.1 & 10.2 & EPA Method 8240 \\
\hline$\mu \mathrm{g} / \mathrm{L}$ & 5.1 & 10.2 & EPA Method 8270 \\
\hline$\mu g / L$ & 5.1 & 10.2 & EPA Method 8270 \\
\hline$\mu g / L$ & 1,665 & 3,330 & EPA Method 8240 \\
\hline$\mu g / L$ & 25 & 50 & EPA Method 8260 \\
\hline$\mu g / L$ & 5 & 10 & EPA Method 8260 \\
\hline$\mu g / L$ & 50 & 100 & Final DWS (CFR, 1995a) \\
\hline$\mu g / L$ & 50 & 100 & Final DWS (CFR, 1995a) \\
\hline$\mu \mathrm{g} / \mathrm{L}$ & 50 & 100 & Final DWS (CFR, 1995a) \\
\hline$\mu \mathrm{Ci} / \mathrm{mL}$ & $3 E-06$ & $6 E-06$ & Interim Final DWS (EPA, 1977) \\
\hline$\mu g / L$ & 0.1 & 0.2 & Proposed DWS (EPA, 1990) \\
\hline$\mu g / L$ & 50 & 100 & EPA Method 6010 \\
\hline$\mu g / L$ & 50 & 100 & EPA Method 6010 \\
\hline$\mu g / L$ & 50 & 100 & EPA Method 6010 \\
\hline$\mu \mathrm{Ci} / \mathrm{mL}$ & $5 E-07$ & $1 E-06$ & Interim Final DWS (EPA, 1977) \\
\hline$\mu \mathrm{Ci} / \mathrm{mL}$ & $4.5 E-06$ & $9 E-06$ & Interim Final DWS (EPA, 1977) \\
\hline$\mu \mathrm{Ci} / \mathrm{mL}$ & $5 E-08$ & $1 E-07$ & Interim Final DWS (EPA, 1977) \\
\hline$\mu g / L$ & $\begin{array}{l}\text { No flag } \\
500\end{array}$ & $\begin{array}{l}\text { No flag } \\
1,000\end{array}$ & $\begin{array}{l}\text { Set by EPD/EMS } \\
\text { Primary DWS (SCDHEC, 1981) }\end{array}$ \\
\hline$\mu g / L$ & 500 & 1,000 & Primary DWS (SCDHEC, 1981) \\
\hline$\mu g / L$ & 500 & 1,000 & Primary DWS (SCDHEC, 1981) \\
\hline$\mu g / L$ & $\begin{array}{l}\text { No flag } \\
50\end{array}$ & $\begin{array}{l}\text { No flag } \\
100\end{array}$ & $\begin{array}{l}\text { Set by EPD/EMS } \\
\text { EPA Method } 8270\end{array}$ \\
\hline$\mu g / L$ & 5 & 10 & EPA Method 8270 \\
\hline$\mu g / L$ & 60 & 120 & EPA Method 8270 \\
\hline$\mu \mathrm{Ci} / \mathrm{mL}$ & $6.65 E-08$ & $1.33 E-07$ & Proposed DWS (EPA, 1991a) \\
\hline$\mu \mathrm{Ci} / \mathrm{mL}$ & 4.15E-09 & 8.3E-09 & Proposed DWS (EPA, 1991a) \\
\hline$\mu \mathrm{Ci} / \mathrm{mL}$ & 4.15E-09 & 8.3E-09 & Proposed DWS (EPA, 1991a) \\
\hline$\mu \mathrm{Ci} / \mathrm{mL}$ & $4.92 E-09$ & $9.84 E-09$ & Proposed DWS (EPA, 1991a) \\
\hline$\mu \mathrm{Ci} / \mathrm{mL}$ & $3.12 E-09$ & $6.23 E-09$ & Proposed DWS (EPA, 1991a) \\
\hline$\mu \mathrm{Ci} / \mathrm{mL}$ & $3.14 \mathrm{E}-09$ & $6.27 E-09$ & Proposed DWS (EPA, 1991a) \\
\hline$\mu g / L$ & 100 & 200 & Final DWS (CFR, 1995a) \\
\hline$\mu g / L$ & 100 & 200 & Final DWS (CFR, 1995a) \\
\hline$\mu g / L$ & 0.55 & 1.1 & EPA Method 8080 \\
\hline$\mu g / L$ & 0.25 & 0.5 & EPA Method 8080 \\
\hline$\mu \mathrm{g} / \mathrm{L}$ & 0.85 & 1.7 & EPA Method 8080 \\
\hline$\mu g / L$ & 81 & 162 & EPA Method 8270 \\
\hline$\mu g / L$ & 0.15 & 0.3 & Proposed DWS (EPA, 1990) \\
\hline$\mu g / L$ & 5 & 10 & EPA Method 8270 \\
\hline$\mu g / L$ & 50 & 100 & Final DWS (CFR, 1995a) \\
\hline$\mu g / L$ & 0.1 & 0.2 & Final DWS (CFR, 1995a) \\
\hline$\mu g / L$ & 0.025 & 0.05 & Final DWS (CFR, 1995a) \\
\hline$\mu g / L$ & 10 & 20 & EPA Method 8240 \\
\hline$\mu g / L$ & $\begin{array}{l}\text { No flag } \\
300\end{array}$ & $\begin{array}{l}\text { No flag } \\
600\end{array}$ & $\begin{array}{l}\text { Set by EPD/EMS } \\
\text { Final DWS (CFR, 1995a) }\end{array}$ \\
\hline$\mu g / L$ & 81 & 162 & EPA Method 8270 \\
\hline$\mu g / L$ & 37.5 & 75 & Final DWS (CFR, 1995a) \\
\hline$\mu g / L$ & 5.1 & 10.2 & EPA Method 8270 \\
\hline$\mu g / L$ & 250 & 500 & EPA Method 8240 \\
\hline
\end{tabular}




\author{
Analyte \\ Dichlorodifluoromethane \\ 1,1-Dichloroethane \\ 1,2-Dichloroethane \\ cis-1,2-Dichloroethylene \\ 1,1-Dichloroethylene \\ 1,2-Dichloroethylene \\ trans-1,2-Dichloroethylene \\ Dichloromethane (Methylene chloride) \\ 2,4-Dichlorophenol \\ 2,6-Dichlorophenol \\ 2,4-Dichlorophenoxyacetic acid \\ 1,2-Dichloropropane \\ 2,2-Dichloropropane \\ cis-1,3-Dichloropropene \\ trans-1,3-Dichloropropene \\ Dieldrin \\ Diethyl phthalate \\ Di(2-ethylhexyl) adipate \\ Dimethoate \\ 2,4-Dimethyl phenol \\ Dimethyl phthalate \\ p-Dimethylaminoazobenzene \\ p-(Dimethylamino)ethylbenzene \\ 7,12-Dimethylbenz [a]anthracene \\ 3,3'-Dimethylbenzidine \\ a,a-Dimethylphenethylamine \\ 1,3-Dinitrobenzene \\ 2,4-Dinitrophenol \\ 2,4-Dinitrotoluene \\ 2,6-Dinitrotoluene \\ Di-n-octyl phthalate \\ 1,4-Dioxane \\ Diphenylamine \\ 1,2-Diphenylhydrazine \\ Diquat dibromide \\ Dissolved organic carbon \\ Disulfoton \\ Eh \\ Endosulfan I \\ Endosulfan II \\ Endosulfan sulfate \\ Endothall \\ Endrin \\ Endrin aldehyde \\ Endrin ketone \\ Ethyl ether \\ Ethyl methacrylate \\ Ethyl methanesulfonate \\ Ethylbenzene \\ Europium-152 \\ Europium-154 \\ Europium-155
}

\begin{tabular}{|c|c|c|}
\hline Unit & Flag 1 & Flag 2 \\
\hline$\mu g / L$ & 10 & 20 \\
\hline$\mu g / L$ & 10 & 20 \\
\hline$\mu g / L$ & 2.5 & 5 \\
\hline$\mu g / L$ & 35 & 70 \\
\hline$\mu g / L$ & 3.5 & 7 \\
\hline$\mu g / L$ & 25 & 50 \\
\hline$\mu g / L$ & 50 & 100 \\
\hline$\mu g / L$ & 2.5 & 5 \\
\hline$\mu g / L$ & 5.1 & 10.2 \\
\hline$\mu g / L$ & 83.5 & 167 \\
\hline$\mu \mathrm{g} / \mathrm{L}$ & 35 & 70 \\
\hline$\mu g / L$ & 2.5 & 5 \\
\hline$\mu \mathrm{g} / \mathrm{L}$ & 5 & 10 \\
\hline$\mu g / L$ & 10 & 20 \\
\hline$\mu \mathrm{g} / \mathrm{L}$ & 10 & 20 \\
\hline$\mu \mathrm{g} / \mathrm{L}$ & 4.15 & 8.3 \\
\hline$\mu \mathrm{g} / \mathrm{L}$ & $\begin{array}{l}\text { No flag } \\
200\end{array}$ & $\begin{array}{l}\text { No flag } \\
400\end{array}$ \\
\hline$\mu g / L$ & 81 & 162 \\
\hline$\mu g / L$ & 5.1 & 10.2 \\
\hline$\mu g / L$ & $\begin{array}{l}\text { No flag } \\
81\end{array}$ & $\begin{array}{l}\text { No flag } \\
162\end{array}$ \\
\hline$\mu g / L$ & 50 & 100 \\
\hline$\mu g / L$ & 81 & 162 \\
\hline$\mu g / L$ & 81 & 162 \\
\hline$\mu g / L$ & 81 & 162 \\
\hline$\mu g / L$ & 81 & 162 \\
\hline$\mu g / L$ & 51 & 102 \\
\hline$\mu g / L$ & 0.5 & 1 \\
\hline$\mu g / L$ & 0.5 & 1 \\
\hline$\mu g / L$ & $\begin{array}{l}\text { No flag } \\
500\end{array}$ & $\begin{array}{l}\text { No flag } \\
1,000\end{array}$ \\
\hline$\mu g / L$ & 81 & 162 \\
\hline$\mu g / L$ & 83.5 & 167 \\
\hline$\mu g / L$ & 10 & 20 \\
\hline$\mu g / L$ & $10,500,000$ & $21,000,000$ \\
\hline$\mu g / L$ & 81 & 162 \\
\hline$\mu g / L$ & $\begin{array}{l}\text { No flag } \\
0.25\end{array}$ & $\begin{array}{l}\text { No flag } \\
0.5\end{array}$ \\
\hline$\mu g / L$ & 0.55 & 1.1 \\
\hline$\mu g / L$ & 0.55 & 1.1 \\
\hline$\mu g / L$ & 50 & 100 \\
\hline$\mu g / L$ & 1 & 2 \\
\hline$\mu g / L$ & 0.85 & 1.7 \\
\hline$\mu g / L$ & $\begin{array}{l}\text { No flag } \\
50\end{array}$ & $\begin{array}{l}\text { No flag } \\
100\end{array}$ \\
\hline$\mu \mathrm{g} / \mathrm{L}$ & 2.5 & 5 \\
\hline$\mu \mathrm{g} / \mathrm{L}$ & 81 & 162 \\
\hline$\mu \mathrm{g} / \mathrm{L}$ & 350 & 700 \\
\hline$\mu \mathrm{Ci} / \mathrm{mL}$ & $3 E-08$ & $6 E-08$ \\
\hline$\mu \mathrm{Ci} / \mathrm{mL}$ & $1 E-07$ & $2 E-07$ \\
\hline$\mu \mathrm{Ci} / \mathrm{mL}$ & $3 E-07$ & $6 E-07$ \\
\hline
\end{tabular}

Source

EPA Method 8240

EPA Method 8240

Final DWS (CFR, 1995a)

Final DWS (CFR, 1995a)

Final DWS (CFR, 1995a)

EPA Method 8240

Final DWS (CFR, 1995a)

Final DWS (CFR, 1995a)

EPA Method 8270

EPA Method 8270

Final DWS (CFR, 1995a)

Final DWS (CFR, 1995a)

EPA Method 8260

EPA Method 8240

EPA Method 8240

EPA Method 8080

Set by EPD/EMS

Final DWS (CFR, 1995a)

EPA Method 8270

EPA Method 8270

Set by EPD/EMS

EPA Method 8270

EPA Method 8270

EPA Method 8270

EPA Method 8270

EPA Method 8270

EPA Method 8270

EPA Method 8270

EPA Method 8270

EPA Method 8270

Set by EPD/EMS

EPA Method 8270

EPA Method 8270

EPA Method 8270

Final DWS (CFR, 1995a)

EPA Method 9060

EPA Method 8270

Set by EPD/EMS

EPA Method 8080

EPA Method 8080

EPA Method 8080

Final DWS (CFR, 1995a)

Final DWS (CFR, 1995a)

EPA Method 8080

Set by EPD/EMS

EPA Method 8260

EPA Method 8270

EPA Method 8270

Final DWS (CFR, 1995a)

Interim Final DWS (EPA, 1977)

Interim Final DWS (EPA, 1977)

Interim Final DWS (EPA, 1977) 


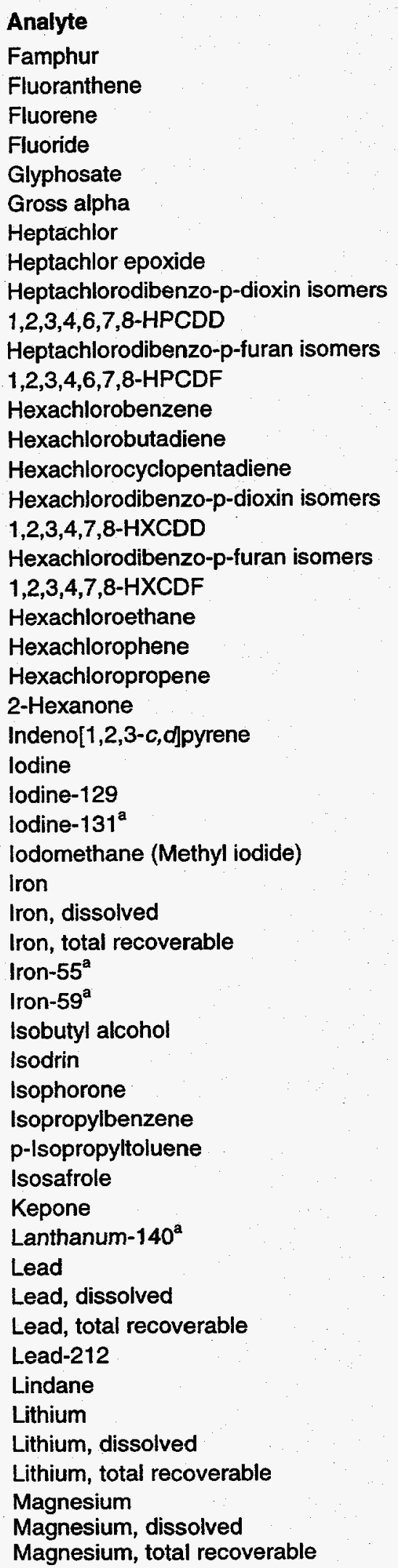

Interim Sanitary Landfill

\begin{tabular}{|c|c|c|c|}
\hline Unit & Flag 1 & Flag 2 & Source \\
\hline$\mu g / L$ & 81 & 162 & EPA Method 8270 \\
\hline$\mu g / L$ & 5.1 & 10.2 & EPA Method 8270 \\
\hline$\mu g / L$ & 5.1 & 10.2 & EPA Method 8270 \\
\hline$\mu \mathrm{g} / \mathrm{L}$ & 2,000 & 4,000 & Final DWS (CFR, 1995a) \\
\hline$\mu g / L$ & 350 & 700 & Final DWS (CFR, 1995a) \\
\hline$\mu \mathrm{Ci} / \mathrm{mL}$ & $7.5 \mathrm{E}-09$ & $1.5 E-08$ & Final DWS (CFR, 1995a) \\
\hline$\mu \mathrm{g} / \mathrm{L}$ & 0.2 & 0.4 & Final DWS (CFR, 1995a) \\
\hline$\mu g / L$ & 0.1 & 0.2 & Final DWS (CFR, 1995a) \\
\hline$\mu g / L$ & 0.007 & 0.014 & EPA Method 8280 \\
\hline$\mu g / L$ & 0.007 & 0.014 & EPA Method 8280 \\
\hline$\mu g / L$ & 0.008 & 0.016 & EPA Method 8280 \\
\hline$\mu g / L$ & 0.008 & 0.016 & EPA Method 8280 \\
\hline$\mu \mathrm{g} / \mathrm{L}$ & 0.5 & 1 & Final DWS (CFR, 1995a) \\
\hline$\mu g / L$ & 5 & 10 & EPA Method 8270 \\
\hline$\mu g / L$ & 25 & 50 & Final DWS (CFR, 1995a) \\
\hline$\mu g / L$ & 0.008 & 0.016 & EPA Method 8280 \\
\hline$\mu g / L$ & 0.0105 & 0.021 & EPA Method 8280 \\
\hline$\mu g / L$ & 0.006 & 0.012 & EPA Method 8280 \\
\hline$\mu g / L$ & 0.0085 & 0.017 & EPA Method 8280 \\
\hline$\mu g / L$ & 0.5 & 1 & EPA Method 8270 \\
\hline$\mu \mathrm{g} / \mathrm{L}$ & 83.5 & 167 & EPA Method 8270 \\
\hline$\mu \mathrm{g} / \mathrm{L}$ & 81 & 162 & EPA Method 8270 \\
\hline$\mu g / L$ & 50 & 100 & EPA Method 8240 \\
\hline$\mu g / L$ & 0.5 & 1 & EPA Method 8270 \\
\hline$\mu g / L$ & 250 & 500 & APHA Method 415A \\
\hline$\mu \mathrm{Ci} / \mathrm{mL}$ & $5 E-10$ & $1 E-09$ & Interim Final DWS (EPA, 1977) \\
\hline$\mu \mathrm{Ci} / \mathrm{mL}$ & $1.5 \mathrm{E}-09$ & $3 E-09$ & Interim Final DWS (EPA, 1977) \\
\hline$\mu \mathrm{g} / \mathrm{L}$ & 125 & 250 & EPA Method 8240 \\
\hline$\mu \mathrm{g} / \mathrm{L}$ & 150 & 300 & Secondary DWS (CFR, 1995b) \\
\hline$\mu g / L$ & 150 & 300 & Secondary DWS (CFR, 1995b) \\
\hline$\mu g / L$ & 150 & 300 & Secondary DWS (CFR, 1995b) \\
\hline$\mu \mathrm{Ci} / \mathrm{mL}$ & $1 E-06$ & $2 E-06$ & Interim Final DWS (EPA, 1977) \\
\hline$\mu \mathrm{Ci} / \mathrm{mL}$ & $1 E-07$ & $2 E-07$ & Interim Final DWS (EPA, 1977) \\
\hline$\mu \mathrm{g} / \mathrm{L}$ & 834.5 & 1,669 & EPA Method 8240 \\
\hline$\mu g / L$ & 81 & 162 & EPA Method 8270 \\
\hline$\mu g / L$ & 5.1 & 10.2 & EPA Method 8270 \\
\hline$\mu g / L$ & 5 & 10 & EPA Method 8260 \\
\hline$\mu g / L$ & 5 & 10 & EPA Method 8260 \\
\hline$\mu g / L$ & 81 & 162 & EPA Method 8270 \\
\hline$\mu g / L$ & 81 & 162 & EPA Method 8270 \\
\hline$\mu \mathrm{Ci} / \mathrm{mL}$ & $3 E-08$ & $6 E-08$ & Interim Final DWS (EPA, 1977) \\
\hline$\mu g / L$ & 25 & 50 & Final DWS (SCDHEC, 1981) \\
\hline$\mu g / L$ & 25 & 50 & Final DWS (SCDHEC, 1981) \\
\hline$\mu g / L$ & 25 & 50 & Final DWS (SCDHEC, 1981) \\
\hline$\mu \mathrm{Ci} / \mathrm{mL}$ & $6.2 E-08$ & $1.23 \mathrm{E}-07$ & Proposed DWS (EPA, 1991a) \\
\hline$\mu \mathrm{g} / \mathrm{L}$ & 0.1 & 0.2 & Final DWS (CFR, 1995a) \\
\hline$\mu g / L$ & 125 & 250 & EPA Method 6010 \\
\hline$\mu \mathrm{g} / \mathrm{L}$ & 125 & 250 & EPA Method 6010 \\
\hline \multirow[t]{2}{*}{$\mu \mathrm{g} / \mathrm{L}$} & 125 & 250 & EPA Method 6010 \\
\hline & $\begin{array}{l}\text { No flag } \\
\text { No flag } \\
\text { No flag }\end{array}$ & $\begin{array}{l}\text { No flag } \\
\text { No flag } \\
\text { No flag }\end{array}$ & $\begin{array}{l}\text { Set by EPD/EMS } \\
\text { Set by EPD/EMS } \\
\text { Set by EPD/EMS }\end{array}$ \\
\hline
\end{tabular}


Analyte

Manganese

Manganese, dissolved

Manganese, total recoverable

Manganese-54

Mercury

Mercury, dissolved

Mercury, total recoverable

Methacrylonitrile

Methapyrilene

Methoxychlor

Methyl tert-butyl ether

Methyl ethyl ketone

Methyl isobutyl ketone

Methyl methacrylate

Methyl methanesulfonate

3-Methylcholanthrene

2-Methyl-4,6-dinitrophenol

2-Methylnaphthalene

Molybdenum

Molybdenum, dissolved

Molybdenum, total recoverable

Naphthalene

1,4-Naphthoquinone

1-Naphthylamine

2-Naphthylamine

Neptunium-237

Neptunium-239

Nickel

Nickel, dissolved

Nickel, total recoverable

Nickel-59

Nickel-63

Niobium-95

Nitrate as nitrogen

Nitrate-nitrite as nitrogen

Nitrite as nitrogen

m-Nitroaniline

o-Nitroaniline

p-Nitroaniline

Nitrobenzene

Nitrogen by Kjeldahl method

2-Nitrophenol

4-Nitrophenol

4-Nitroquinoline-1-oxide

N-Nitrosodi-n-butylamine

$\mathrm{N}$-Nitrosodiethylamine

$\mathrm{N}$-Nitrosodimethylamine

$\mathrm{N}$-Nitrosodiphenylamine

$\mathrm{N}$-Nitrosodipropylamine

$\mathrm{N}$-Nitrosomethylethylamine

$\mathrm{N}$-Nitrosomorpholine

$\mathrm{N}$-Nitrosopiperidine

Interim Sanitary Landfill

\begin{tabular}{|c|c|c|c|}
\hline Unit & Flag 1 & Flag 2 & Source \\
\hline$\mu g / L$ & 25 & 50 & Secondary DWS (CFR, 1995b) \\
\hline$\mu g / L$ & 25 & 50 & Secondary DWS (CFR, 1995b) \\
\hline$\mu g / L$ & 25 & 50 & Secondary DWS (CFR, 1995b) \\
\hline$\mu \mathrm{Ci} / \mathrm{mL}$ & $1.5 E-07$ & $3 E-07$ & Interim Final DWS (EPA, 1977) \\
\hline$\mu g / L$ & 1 & 2 & Final DWS (CFR, 1995a) \\
\hline$\mu g / L$ & 1 & 2 & Final DWS (CFR, 1995a) \\
\hline$\mu g / L$ & 1 & 2 & Final DWS (CFR, 1995a) \\
\hline$\mu \mathrm{g} / \mathrm{L}$ & 416.5 & 833 & EPA Method 8240 \\
\hline$\mu g / L$ & 81 & 162 & EPA Method 8270 \\
\hline$\mu \mathrm{g} / \mathrm{L}$ & 20 & 40 & Final DWS (CFR, 1995a) \\
\hline$\mu g / L$ & 5 & 10 & EPA Method 8260 \\
\hline & $\begin{array}{l}\text { No flag } \\
\text { No flag }\end{array}$ & $\begin{array}{l}\text { No flag } \\
\text { No flag }\end{array}$ & $\begin{array}{l}\text { Set by EPD/EMS } \\
\text { Set by EPD/EMS }\end{array}$ \\
\hline$\mu \mathrm{g} / \mathrm{L}$ & 50 & 100 & EPA Method 8270 \\
\hline$\mu \mathrm{g} / \mathrm{L}$ & 81 & 162 & EPA Method 8270 \\
\hline$\mu \mathrm{g} / \mathrm{L}$ & 81 & 162 & EPA Method 8270 \\
\hline$\mu \mathrm{g} / \mathrm{L}$ & 51 & 102 & EPA Method 8270 \\
\hline$\mu g / L$ & 5 & 10 & EPA Method 8270 \\
\hline$\mu g / L$ & 250 & 500 & EPA Method 6010 \\
\hline$\mu g / L$ & 250 & 500 & EPA Method 6010 \\
\hline$\mu g / \mathrm{L}$ & 250 & 500 & EPA Method 6010 \\
\hline$\mu g / L$ & 83.5 & 167 & EPA Method 8270 \\
\hline$\mu \mathrm{g} / \mathrm{L}$ & 81 & 162 & EPA Method 8270 \\
\hline$\mu g / L$ & 81 & 162 & EPA Method 8270 \\
\hline$\mu g / L$ & 81 & 162 & EPA Method 8270 \\
\hline$\mu \mathrm{Ci} / \mathrm{mL}$ & $3.53 E-09$ & 7.06E-09 & Proposed DWS (EPA, 1991a) \\
\hline$\mu \mathrm{Ci} / \mathrm{mL}$ & $8.4 E-07$ & $1.68 E-06$ & Proposed DWS (EPA, 1991a) \\
\hline$\mu g / L$ & 50 & 100 & Final DWS (CFR, 1995a) \\
\hline$\mu g / L$ & 50 & 100 & Final DWS (CFR, 1995a) \\
\hline$\mu g / L$ & 50 & 100 & Final DWS (CFR, 1995a) \\
\hline$\mu \mathrm{Ci} / \mathrm{mL}$ & $1.5 \mathrm{E}-07$ & $3 E-07$ & Interim Final DWS (EPA, 1977) \\
\hline$\mu \mathrm{Ci} / \mathrm{mL}$ & $2.5 E-08$ & $5 E-08$ & Interim Final DWS (EPA, 1977) \\
\hline$\mu \mathrm{Ci} / \mathrm{mL}$ & $1.5 \mathrm{E}-07$ & $3 E-07$ & Interim Final DWS (EPA, 1977) \\
\hline$\mu g / L$ & 5,000 & 10,000 & Final DWS (CFR, 1995a) \\
\hline$\mu \mathrm{g} / \mathrm{L}$ & 5,000 & 10,000 & Final DWS (CFR, 1995a) \\
\hline$\mu g / L$ & 500 & 1,000 & Final DWS (CFR, 1995a) \\
\hline$\mu g / L$ & 5 & 10 & EPA Method 8270 \\
\hline$\mu g / L$ & 5 & 10 & EPA Method 8270 \\
\hline$\mu \mathrm{g} / \mathrm{L}$ & 5 & 10 & EPA Method 8270 \\
\hline$\mu g / L$ & 5.1 & 10.2 & EPA Method 8270 \\
\hline$\mu g / L$ & 500 & 1,000 & EPA Method 351.2 \\
\hline$\mu g / L$ & 5.1 & 10.2 & EPA Method 8270 \\
\hline$\mu g / L$ & 5.1 & 10.2 & EPA Method 8270 \\
\hline$\mu g / L$ & 81 & 162 & EPA Method 8270 \\
\hline$\mu g / L$ & 81 & 162 & EPA Method 8270 \\
\hline$\mu g / L$ & 81 & 162 & EPA Method 8270 \\
\hline$\mu g / L$ & 83.5 & 167 & EPA Method 8270 \\
\hline$\mu g / L$ & 5.1 & 10.2 & EPA Method 8270 \\
\hline$\mu g / L$ & 5.1 & 10.2 & EPA Method 8270 \\
\hline$\mu g / L$ & 81 & 162 & EPA Method 8270 \\
\hline$\mu g / L$ & 81 & 162 & EPA Method 8270 \\
\hline$\mu g / L$ & 81 & 162 & EPA Method 8270 \\
\hline
\end{tabular}

1996 Annual Report 
Analyte

N-Nitrosopyrrolidine

5-Nitro-0-toluidine

Nonvolatile beta

Octachlorodibenzo-p-dioxin isomers

Octachlorodibenzo-p-furan isomers

Odor

Oil and grease

Oxamyl

Parathion

Parathion methyl

PCB 1016

PCB 1221

PCB 1232

PCB 1242

PCB 1248

PCB 1254

PCB 1260

PCB 1262

Pentachlorobenzene

Pentachlorodibenzo-p-dioxin isomers

1,2,3,7,8-PCDD

Pentachlorodibenzo-p-furan isomers

1,2,3,7,8-PCDF

Pentachloroethane

Pentachloronitrobenzene

Pentachlorophenol

$\mathrm{pH}^{\mathrm{o}}$

$\mathrm{pH}^{\mathrm{d}}$

Phenacetin

Phenanthrene

Phenol

Phenols

p-Phenylenediamine

Phorate

Picloram

2-Picoline

Plutonium-238

Plutonium-239

Plutonium-239/240

Plutonium-240

Plutonium-241 ${ }^{a}$

Plutonium-242

Potassium

Potassium, dissolved

Potassium, total recoverable

Potassium -40

Promethium-144

Promethium-146

Promethium-147

Pronamid

Propionitrile

n-Propylbenzene

\begin{tabular}{|c|c|c|}
\hline Unit & Flag 1 & Flag 2 \\
\hline$\mu g / L$ & 81 & 162 \\
\hline$\mu g / L$ & 81 & 162 \\
\hline$\mu \mathrm{Ci} / \mathrm{mL}$ & $2.5 E-08$ & $5 E-08$ \\
\hline$\mu g / L$ & 0.0085 & 0.017 \\
\hline$\mu g / L$ & 0.0065 & 0.013 \\
\hline$\mu g / L$ & $\begin{array}{l}\text { No flag } \\
8,350\end{array}$ & $\begin{array}{l}\text { No flag } \\
16,700\end{array}$ \\
\hline$\mu g / L$ & 100 & 200 \\
\hline$\mu g / L$ & 0.4 & 0.8 \\
\hline$\mu g / L$ & 0.4 & 0.8 \\
\hline$\mu \mathrm{g} / \mathrm{L}$ & 0.25 & 0.5 \\
\hline$\mu g / L$ & 0.25 & 0.5 \\
\hline$\mu g / L$ & 0.25 & 0.5 \\
\hline$\mu g / L$ & 0.25 & 0.5 \\
\hline$\mu g / L$ & 0.25 & 0.5 \\
\hline$\mu g / L$ & 0.25 & 0.5 \\
\hline$\mu \mathrm{g} / \mathrm{L}$ & 0.25 & 0.5 \\
\hline$\mu g / L$ & 0.25 & 0.5 \\
\hline$\mu g / L$ & 81 & 162 \\
\hline$\mu g / L$ & 0.008 & 0.016 \\
\hline$\mu g / L$ & 0.0075 & 0.015 \\
\hline$\mu g / L$ & 0.0085 & 0.017 \\
\hline$\mu g / L$ & 0.0085 & 0.017 \\
\hline$\mu g / L$ & 81 & 162 \\
\hline$\mu g / L$ & 81 & 162 \\
\hline$\mu g / L$ & 0.5 & 1 \\
\hline $\begin{array}{l}\mathrm{pH} \\
\mathrm{pH}\end{array}$ & $\begin{array}{l}8 \\
4\end{array}$ & $\begin{array}{l}10 \\
3\end{array}$ \\
\hline$\mu \mathrm{g} / \mathrm{L}$ & 81 & 162 \\
\hline$\mu g / L$ & 5.1 & 10.2 \\
\hline$\mu \mathrm{g} / \mathrm{L}$ & 83.5 & 167 \\
\hline$\mu g / L$ & 50 & 100 \\
\hline$\mu \mathrm{g} / \mathrm{L}$ & 81 & 162 \\
\hline$\mu g / L$ & 0.85 & 1.7 \\
\hline$\mu g / L$ & 250 & 500 \\
\hline$\mu g / L$ & 81 & 162 \\
\hline$\mu \mathrm{Ci} / \mathrm{mL}$ & $3.51 E-09$ & $7.02 E-09$ \\
\hline$\mu \mathrm{Ci} / \mathrm{mL}$ & $3.11 E-08$ & $6.21 E-08$ \\
\hline$\mu \mathrm{Ci} / \mathrm{mL}$ & $3.11 E-08$ & $6.21 E-08$ \\
\hline$\mu \mathrm{Ci} / \mathrm{mL}$ & $3.11 E-08$ & $6.22 E-08$ \\
\hline$\mu \mathrm{Ci} / \mathrm{mL}$ & $3.13 E-08$ & $6.26 \mathrm{E}-08$ \\
\hline$\mu \mathrm{Ci} / \mathrm{mL}$ & $3.27 E-08$ & $6.54 E-08$ \\
\hline$\mu \mathrm{Ci} / \mathrm{mL}$ & $\begin{array}{l}\text { No flag } \\
\text { No flag } \\
\text { No flag } \\
1.5 E-07\end{array}$ & $\begin{array}{l}\text { No flag } \\
\text { No flag } \\
\text { No flag } \\
3 E-07\end{array}$ \\
\hline$\mu \mathrm{Ci} / \mathrm{mL}$ & $5 E-08$ & $1 E-07$ \\
\hline$\mu \mathrm{Ci} / \mathrm{mL}$ & $5 E-08$ & $1 E-07$ \\
\hline$\mu \mathrm{Ci} / \mathrm{mL}$ & $2.62 E-06$ & $5.24 \mathrm{E}-06$ \\
\hline$\mu g / L$ & 81 & 162 \\
\hline$\mu g / L$ & 1,665 & 3,330 \\
\hline$\mu g / L$ & 5 & 10 \\
\hline
\end{tabular}

Source

EPA Method 8270

EPA Method 8270

Interim Final DWS (EPA, 1977)

EPA Method 8280

EPA Method 8280

Set by EPD/EMS

EPA Method 413.1

Final DWS (CFR, 1995a)

EPA Method 8080

EPA Method 8080

Final DWS (CFR, 1995a)

Final DWS (CFR, 1995a)

Final DWS (CFR, 1995a)

Final DWS (CFR, 1995a)

Final DWS (CFR, 1995a)

Final DWS (CFR, 1995a)

Final DWS (CFR, 1995a)

Final DWS (CFR, 1995a)

EPA Method 8270

EPA Method 8280

EPA Method 8280

EPA Method 8280

EPA Method 8280

EPA Method 8270

EPA Method 8270

Final DWS (CFR, 1995a)

Set by EPD/EMS

Set by EPD/EMS

EPA Method 8270

EPA Method 8270

EPA Method 8270

EPA Method 420.1

EPA Method 8270

EPA Method 8080

Final DWS (CFR, 1995a)

EPA Method 8270

Proposed DWS (EPA, 1991a)

Proposed DWS (EPA, 1991a)

Proposed DWS (EPA, 1991a)

Proposed DWS (EPA, 1991a)

Proposed DWS (EPA, 1991a)

Proposed DWS (EPA, 1991a)

Set by EPD/EMS

Set by EPD/EMS

Set by EPD/EMS

Proposed DWS (EPA, 1986a)

EPA Method 901.1

EPA Method 901.1

Proposed DWS (EPA, 1991a)

EPA Method 8270

EPA Method 8240

EPA Method 8260 


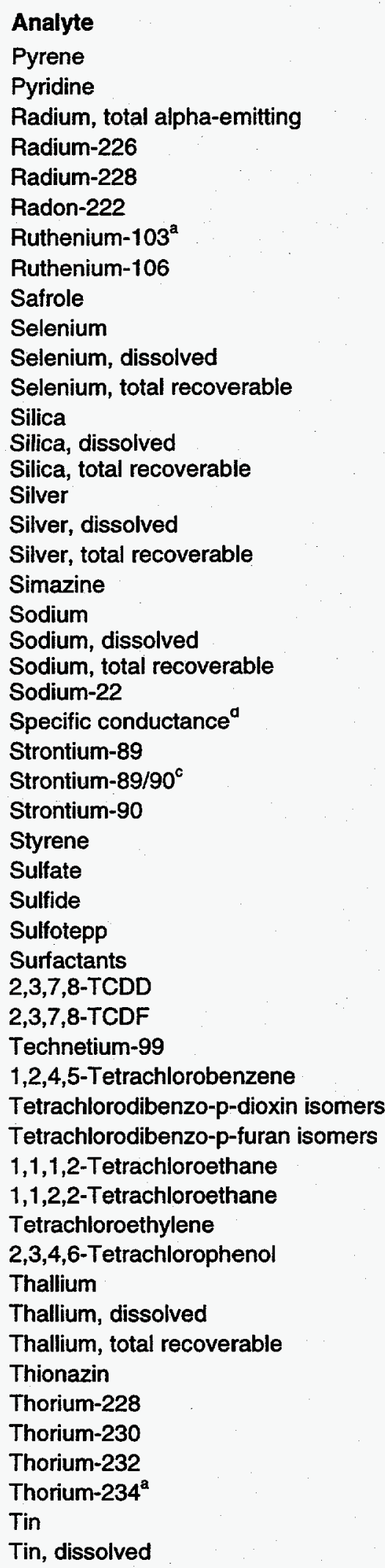

\begin{tabular}{|c|c|c|}
\hline Unit & Flag 1 & Flag 2 \\
\hline$\mu \mathrm{g} / \mathrm{L}$ & 5.1 & 10.2 \\
\hline$\mu g / L$ & 81 & 162 \\
\hline$\mu \mathrm{Ci} / \mathrm{mL}$ & $1 E-08$ & $2 E-08$ \\
\hline$\mu \mathrm{Ci} / \mathrm{mL}$ & $1 E-08$ & $2 E-08$ \\
\hline$\mu \mathrm{Ci} / \mathrm{mL}$ & $1 E-08$ & $2 E-08$ \\
\hline$\mu \mathrm{Ci} / \mathrm{mL}$ & $1.5 E-07$ & $3 E-07$ \\
\hline$\mu \mathrm{Ci} / \mathrm{mL}$ & $1 E-07$ & $2 E-07$ \\
\hline$\mu \mathrm{Ci} / \mathrm{mL}$ & $1.5 \mathrm{E}-08$ & $3 E-08$ \\
\hline$\mu g / L$ & 81 & 162 \\
\hline$\mu \mathrm{g} / \mathrm{L}$ & 25 & 50 \\
\hline$\mu g / L$ & 25 & 50 \\
\hline$\mu g / L$ & 25 & 50 \\
\hline$\mu g / L$ & $\begin{array}{l}\text { No flag } \\
\text { No flag } \\
\text { No flag } \\
50\end{array}$ & $\begin{array}{l}\text { No flag } \\
\text { No flag } \\
\text { No flag } \\
100\end{array}$ \\
\hline$\mu g / L$ & 50 & 100 \\
\hline$\mu g^{\prime} L$ & 50 & 100 \\
\hline$\mu g / L$ & 2 & 4 \\
\hline$\mu \mathrm{Ci} / \mathrm{mL}$ & $\begin{array}{l}\text { No flag } \\
\text { No flag } \\
\text { No flag } \\
2.33 E-07\end{array}$ & $\begin{array}{l}\text { No flag } \\
\text { No flag } \\
\text { No flag } \\
4.66 E-07\end{array}$ \\
\hline$\mu \mathrm{S} / \mathrm{cm}$ & 250 & 500 \\
\hline$\mu \mathrm{Ci} / \mathrm{mL}$ & $1 E-08$ & $2 E-08$ \\
\hline$\mu \mathrm{Ci} / \mathrm{mL}$ & $4 \mathrm{E}-09$ & $8 E-09$ \\
\hline$\mu \mathrm{Ci} / \mathrm{mL}$ & $4 E-09$ & $8 E-09$ \\
\hline$\mu g / L$ & 50 & 100 \\
\hline$\mu \mathrm{g} / \mathrm{L}$ & 200,000 & 400,000 \\
\hline$\mu g / L$ & 8,350 & 16,700 \\
\hline$\mu g / L$ & 81 & 162 \\
\hline$\mu g / L$ & $\begin{array}{l}\text { No flag } \\
0.007\end{array}$ & $\begin{array}{l}\text { No flag } \\
0.014\end{array}$ \\
\hline$\mu g / L$ & 0.00425 & 0.0085 \\
\hline$\mu \mathrm{Ci} / \mathrm{mL}$ & $4.5 E-07$ & $9 E-07$ \\
\hline$\mu g / L$ & 81 & 162 \\
\hline$\mu g / L$ & 0.007 & 0.014 \\
\hline$\mu g / L$ & 0.0055 & 0.011 \\
\hline$\mu g / L$ & 10 & 20 \\
\hline$\mu g / L$ & 50 & 100 \\
\hline$\mu g / L$ & 2.5 & 5 \\
\hline$\mu g / L$ & 83.5 & 167 \\
\hline$\mu g / L$ & 1 & 2 \\
\hline$\mu g / L$ & 1 & 2 \\
\hline$\mu g / L$ & 1 & 2 \\
\hline$\mu g / L$ & 81 & 162 \\
\hline$\mu \mathrm{Ci} / \mathrm{mL}$ & $6.25 \mathrm{E}-08$ & $1.25 E-07$ \\
\hline$\mu \mathrm{Ci} / \mathrm{mL}$ & $3.96 E-08$ & $7.92 E-08$ \\
\hline$\mu \mathrm{Ci} / \mathrm{mL}$ & 4.4E-08 & $8.8 E-08$ \\
\hline$\mu \mathrm{Ci} / \mathrm{mL}$ & $2 E-07$ & $4.01 E-07$ \\
\hline$\mu g / L$ & 250 & 500 \\
\hline$\mu g / L$ & 250 & 500 \\
\hline
\end{tabular}

Source

EPA Method 8270

EPA Method 8270

Proposed DWS (EPA, 1991a)

Proposed DWS (EPA, 1991a)

Proposed DWS (EPA, 1991a)

Proposed DWS (EPA, 1991a)

Interim Final DWS (EPA, 1977)

Interim Final DWS (EPA, 1977)

EPA Method 8270

Final DWS (CFR, 1995a)

Final DWS (CFR, 1995a)

Final DWS (CFR, 1995a)

Set by EPD/EMS

Set by EPD/EMS

Set by EPD/EMS

Secondary DWS (CFR, 1995b)

Secondary DWS (CFR, 1995b)

Secondary DWS (CFR, 1995b)

Final DWS (CFR, 1995a)

Set by EPD/EMS

Set by EPD/EMS

Set by EPD/EMS

Proposed DWS (EPA, 1991a)

Set by EPD/EMS

Interim Final DWS (EPA, 1977)

Final DWS (CFR, 1995a)

Final DWS (CFR, 1995a)

Final DWS (CFR, 1995a)

Proposed DWS (EPA, 1990)

EPA Method 9030

EPA Method 8270

Set by EPD/EMS

Final DWS (CFR, 1995a)

EPA Method 8280

Interim Final DWS (EPA, 1977)

EPA Method 8270

EPA Method 8280

EPA Method 8280

EPA Method 8240

EPA Method 8240

Final DWS (CFR, 1995a)

EPA Method 8270

Final DWS (CFR, 1995a)

Final DWS (CFR, 1995a)

Final DWS (CFR, 1995a)

EPA Method 8270

Proposed DWS (EPA, 1991a)

Proposed DWS (EPA, 1991a)

Proposed DWS (EPA, 1991a)

Proposed DWS (EPA, 1991a)

EPA Method 282.2

EPA Method 282.2 
Analyte

Tin, total recoverable

Tin-113

Toluene

o-Toluidine

Total carbon

Total coliform

Total dissolved solids

Total hydrocarbons

Total inorganic carbon

Total organic carbon

Total organic halogens

Total organic nitrogen

Total petroleum hydrocarbons

Total phosphates (as P)

Total phosphorus

Toxaphene

2,4,5-TP (Silvex)

Tributyl phosphate

1,2,3-Trichlorobenzene

1,2,4-Trichlorobenzene

1,1,1-Trichloroethane

1,1,2-Trichloroethane

Trichloroethylene

Trichlorofluoromethane

2,4,5-Trichlorophenol

2,4,6-Trichlorophenol

2,4,5-Trichlorophenoxyacetic acid

1,2,3-Trichloropropane

Trichlorotrifluoroethane

$0,0,0$-Triethyl phosphorothioate

1,2,4-Trimethylbenzene

1,3,5-Trimethylbenzene

1,3,5-Trinitrobenzene

Tritium

Turbidity ${ }^{e}$

Uranium

Uranium alpha activity

Uranium, dissolved

Uranium, total recoverable

Uranium-233/234 ${ }^{\circ}$

Uranium-234

Uranium-235

Uranium-238

Vanadium

Vanadium, dissolved

Vanadium, total recoverable

Vinyl acetate

m/p-Xylene

o-Xylene

Xylenes

Yttrium-88

Zine

Interim Sanitary Landfill

\begin{tabular}{|c|c|c|}
\hline Unit & Flag 1 & Flag 2 \\
\hline$\mu g / L$ & 250 & 500 \\
\hline$\mu \mathrm{Ci} / \mathrm{mL}$ & $1.5 E-07$ & $3 E-07$ \\
\hline$\mu g / L$ & 500 & 1,000 \\
\hline$\mu g / L$ & 81 & 162 \\
\hline$\mu g / L$ & 5,000 & 10,000 \\
\hline $\begin{array}{l}\text { N/A } \\
\mu g / L\end{array}$ & $\begin{array}{l}0 \\
\text { No flag } \\
5,000\end{array}$ & $\begin{array}{l}0 \\
\text { No flag } \\
10,000\end{array}$ \\
\hline$\mu g / L$ & 8,350 & 16,700 \\
\hline$\mu g / L$ & 500,000 & $1,000,000$ \\
\hline$\mu g^{\prime} L$ & 50 & 100 \\
\hline$\mu g / L$ & 500 & 1,000 \\
\hline$\mu g / L$ & 8,350 & 16,700 \\
\hline$\mu g / L$ & $\begin{array}{l}\text { No flag } \\
\text { No flag } \\
1.5\end{array}$ & $\begin{array}{l}\text { No flag } \\
\text { No flag } \\
3\end{array}$ \\
\hline$\mu g / L$ & 25 & 50 \\
\hline$\mu g / L$ & 86 & 172 \\
\hline$\mu g / L$ & 5 & 10 \\
\hline$\mu g / L$ & 35 & 70 \\
\hline$\mu g / L$ & 100 & 200 \\
\hline$\mu g / L$ & 2.5 & 5 \\
\hline$\mu g / L$ & 2.5 & 5 \\
\hline$\mu g / L$ & 10 & 20 \\
\hline$\mu g / L$ & 5 & 10 \\
\hline$\mu g / L$ & 0.5 & 1 \\
\hline$\mu g / L$ & 0.25 & 0.5 \\
\hline$\mu g / L$ & 10 & 20 \\
\hline$\mu g / L$ & 50 & 100 \\
\hline$\mu g / L$ & 81 & 162 \\
\hline$\mu \mathrm{g} / \mathrm{L}$ & 5 & 10 \\
\hline$\mu g / L$ & 5 & 10 \\
\hline$\mu g / L$ & 81 & 162 \\
\hline$\mu \mathrm{Ci} / \mathrm{mL}$ & $1 E-05$ & $2 E-05$ \\
\hline$\mu g / L$ & $\begin{array}{l}\text { No flag } \\
10\end{array}$ & $\begin{array}{l}\text { No flag } \\
20\end{array}$ \\
\hline$\mu \mathrm{Ci} / \mathrm{mL}$ & $1.5 E-08$ & $3 E-013$ \\
\hline$\mu g / L$ & 10 & 20 \\
\hline$\mu g / L$ & 10 & 20 \\
\hline$\mu \mathrm{Ci} / \mathrm{mL}$ & $6.9 \mathrm{E}-09$ & $1.38 E-08$ \\
\hline$\mu \mathrm{Ci} / \mathrm{mL}$ & $6.95 \mathrm{E}-09$ & $1.39 \mathrm{E}-08$ \\
\hline$\mu \mathrm{Ci} / \mathrm{mL}$ & $7.25 \mathrm{E}-09$ & $1.45 \mathrm{E}-08$ \\
\hline$\mu \mathrm{Ci} / \mathrm{mL}$ & 7.3E-09 & $1.46 \mathrm{E}-08$ \\
\hline$\mu g / L$ & 66.5 & 133 \\
\hline$\mu g / L$ & 66.5 & 133 \\
\hline$\mu g / L$ & 66.5 & 133 \\
\hline$\mu g / L$ & 50 & 100 \\
\hline$\mu g / L$ & 81 & 162 \\
\hline$\mu g / L$ & 5 & 10 \\
\hline $\begin{array}{l}\mu \mathrm{g} / \mathrm{L} \\
\mu \mathrm{Ci} / \mathrm{mL}\end{array}$ & $\begin{array}{l}5,000 \\
5 E-08\end{array}$ & $\begin{array}{l}10,000 \\
1 E-07\end{array}$ \\
\hline$\mu g / L$ & 2,500 & 5,000 \\
\hline
\end{tabular}

Source

EPA Method 282.2

Interim Final DWS (EPA, 1977)

Final DWS (CFR, 1995a)

EPA Method 8270

EPA Method 9060

Final DWS (CFR, 1995a)

Set by EPD/EMS

EPA Method 418.1

EPA Method 9060

EPA Method 9060

EPA Method 9020

APHA Method 420

EPA Method 418.1

Set by EPD/EMS

Set by EPD/EMS

Final DWS (CFR, 1995a)

Final DWS (CFR, 1995a)

EPA Method 8270

EPA Method 8260

Final DWS (CFR, 1995a)

Final DWS (CFR, 1995a)

Final DWS (CFR, 1995a)

Final DWS (CFR, 1995a)

EPA Method 8240

EPA Method 8270

EPA Method 8270

EPA Method 8150

EPA Method 8240

EPA Method 8260

EPA Method 8270

EPA Method 8260

EPA Method 8260

EPA Method 8270

Final DWS (CFR, 1995a)

Set by EPD/EMS

Proposed DWS (EPA, 1991a)

Proposed DWS (EPA, 1991a)

Proposed DWS (EPA, 1991a)

Proposed DWS (EPA, 1991a)

Proposed DWS (EPA, 1991a)

Proposed DWS (EPA, 1991a)

Proposed DWS (EPA, 1991a)

Proposed DWS (EPA, 1991a)

EPA Method 6010

EPA Method 6010

EPA Method 6010

EPA Method 8240

EPA Method 8260

EPA Method 8260

Final DWS (CFR, 1995a)

EPA Method 901.1

Secondary DWS (CFR, 1995b) 
Analyte

Zinc, dissolved

Zinc, total recoverable

Zinc-65

Zirconium-95

Zirconium/Niobium-95

$\begin{array}{llll}\text { Unit } & \text { Flag 1 } & \text { Flag 2 } & \text { Source } \\ \mu g / L & 2,500 & 5,000 & \text { Secondary DWS (CFR, 1995b) } \\ \mu g / L & 2,500 & 5,000 & \text { Secondary DWS (CFR, 1995b) } \\ \mu \mathrm{Ci} / \mathrm{mL} & 1.5 \mathrm{E}-07 & 3 \mathrm{E}-07 & \text { Interim Final DWS (EPA, 1977) } \\ \mu \mathrm{Ci} / \mathrm{mL} & 1 \mathrm{E}-07 & 2 \mathrm{E}-07 & \text { Interim Final DWS (EPA, 1977) } \\ \mu \mathrm{Ci} / \mathrm{mL} & 1 \mathrm{E}-07 & 2 \mathrm{E}-07 & \text { Interim Final DWS (EPA, 1977) }\end{array}$

Unit Flag 1

Flag 2

a EMS discontinued monitoring this radionuclide because it is inappropriate for the SRS groundwater monitoring program.

b EPD/EMS set this flagging criterion using the 1991 proposed DWS because the final DWS in 1977 may have been in error.

c When radionuclide analyses are combined, the lowest DWS of the two isotopes is used for flagging.

d Will not trigger scheduling.

a The primary maximum contaminant level range for turbidity is 1-5 NTU, which is inappropriate for the SRS groundwater monitoring program. 
WSRC-TR-96-0186-4

Unclassified

THIS PAGE LEFT BLANK INTENTIONALLY. 
WSRC-TR-96-0186-4

Unclassified

\section{Appendix C}

Figures 
WSRC-TR-96-0186-4

Unclassified

THIS PAGE LEFT BLANK INTENTIONALLY. 


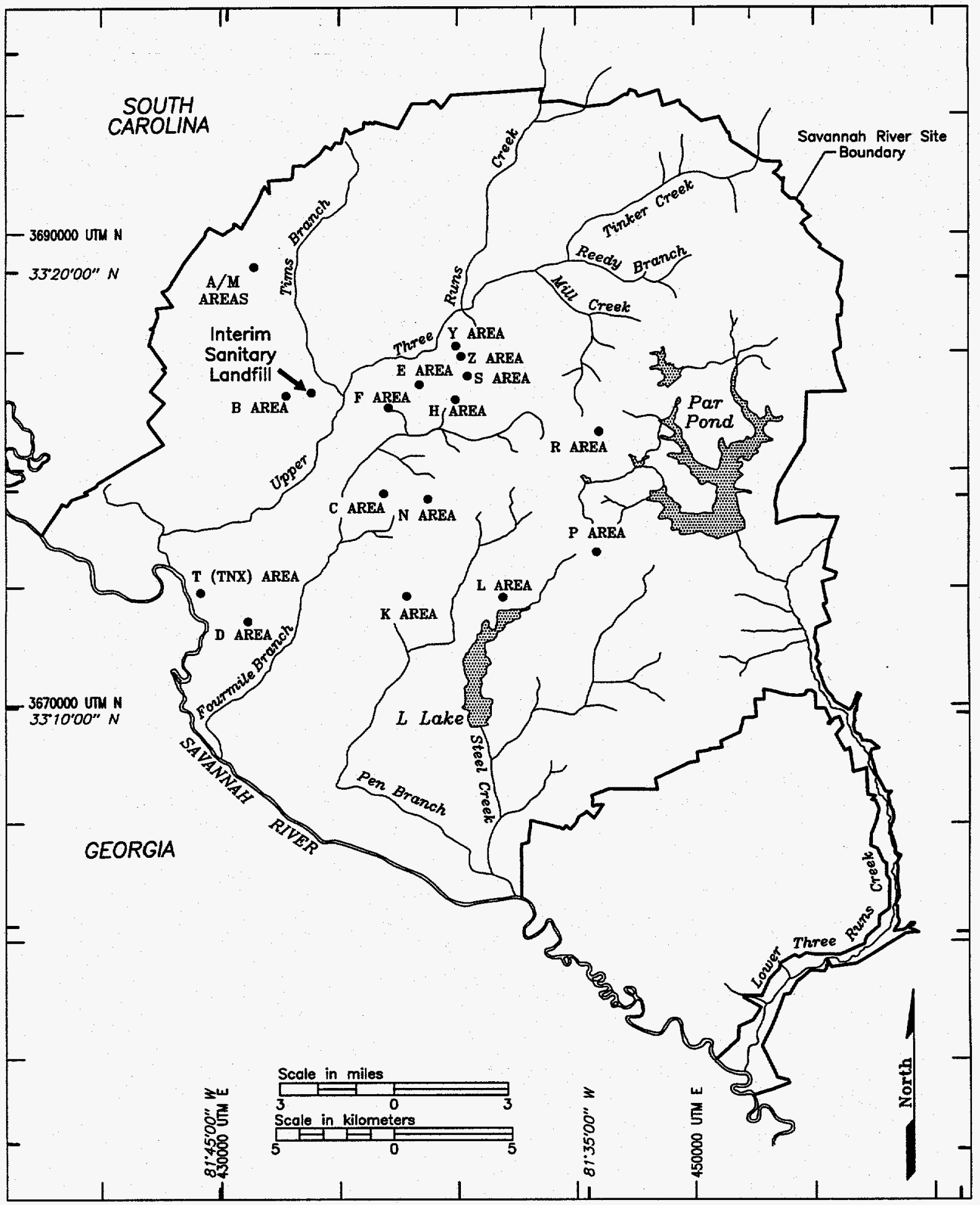

Figure 1. Location of the Interim Sanitary Landfill at the Savannah River Site 


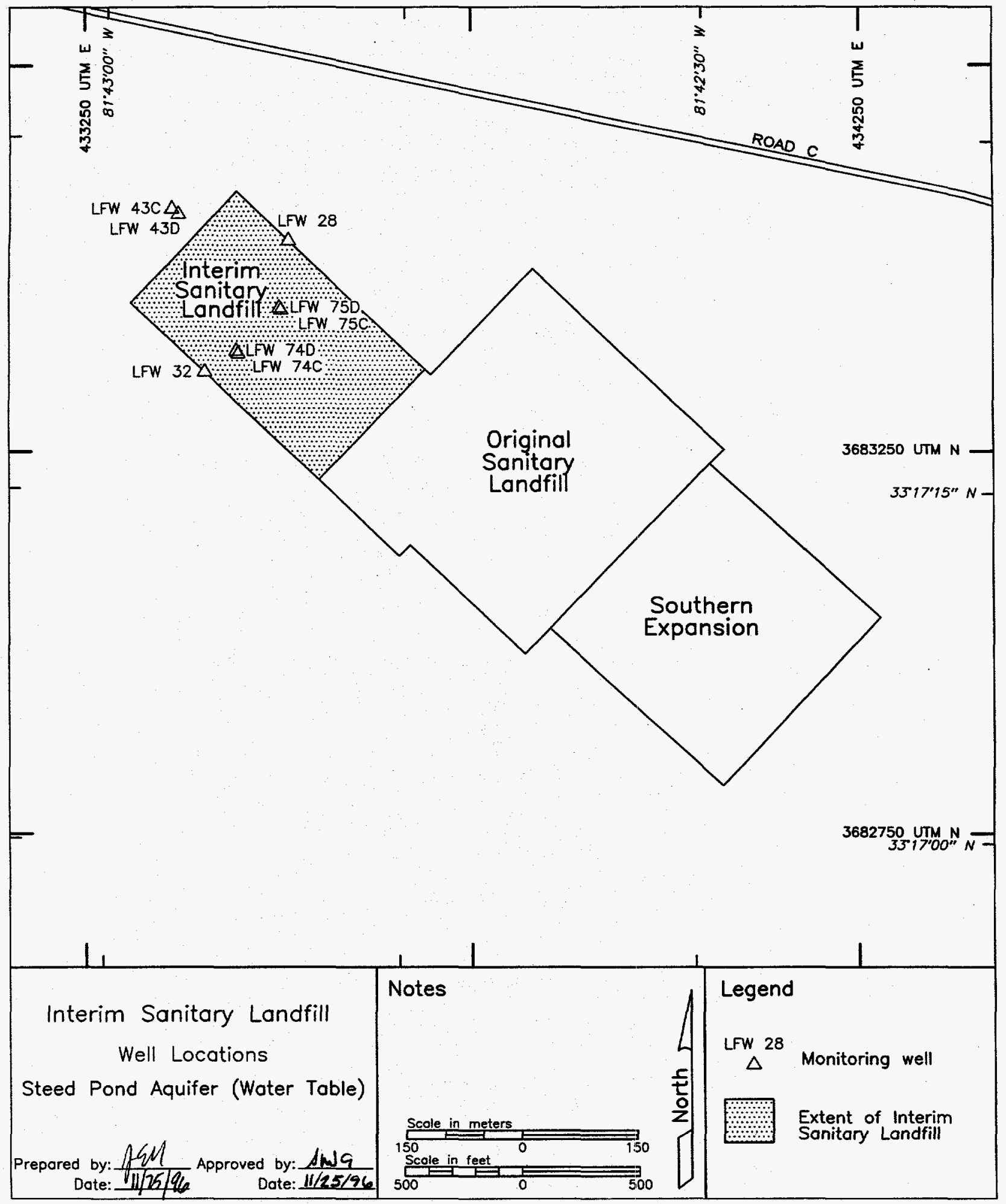

Figure 2. Location of Monitoring Wells at the Interim Sanitary Landfill 


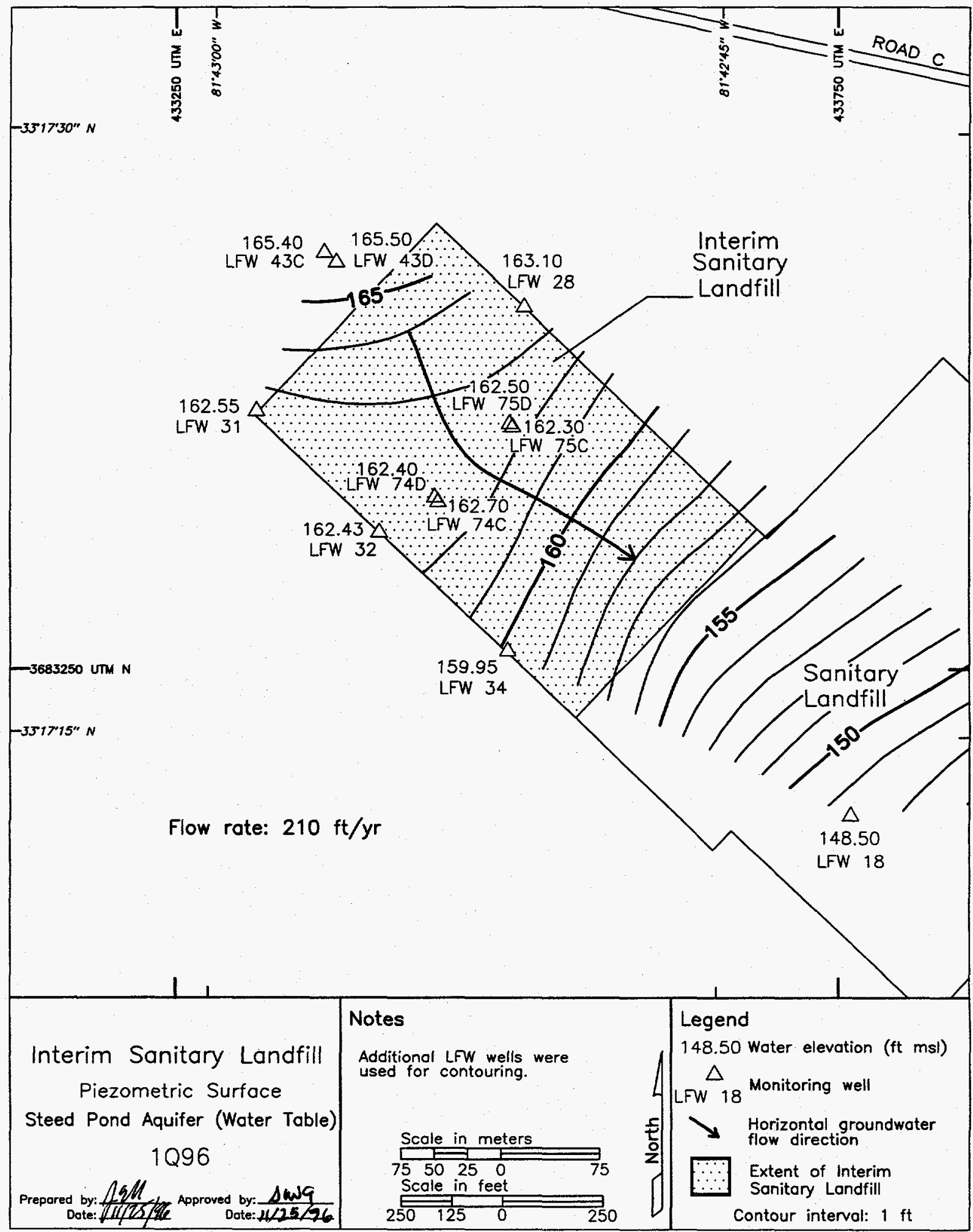

Figure 3. Piezometric Surface Map of Steed Pond Aquifer (Water Table) at the Interim Sanitary Landfill, First Quarter 1996 


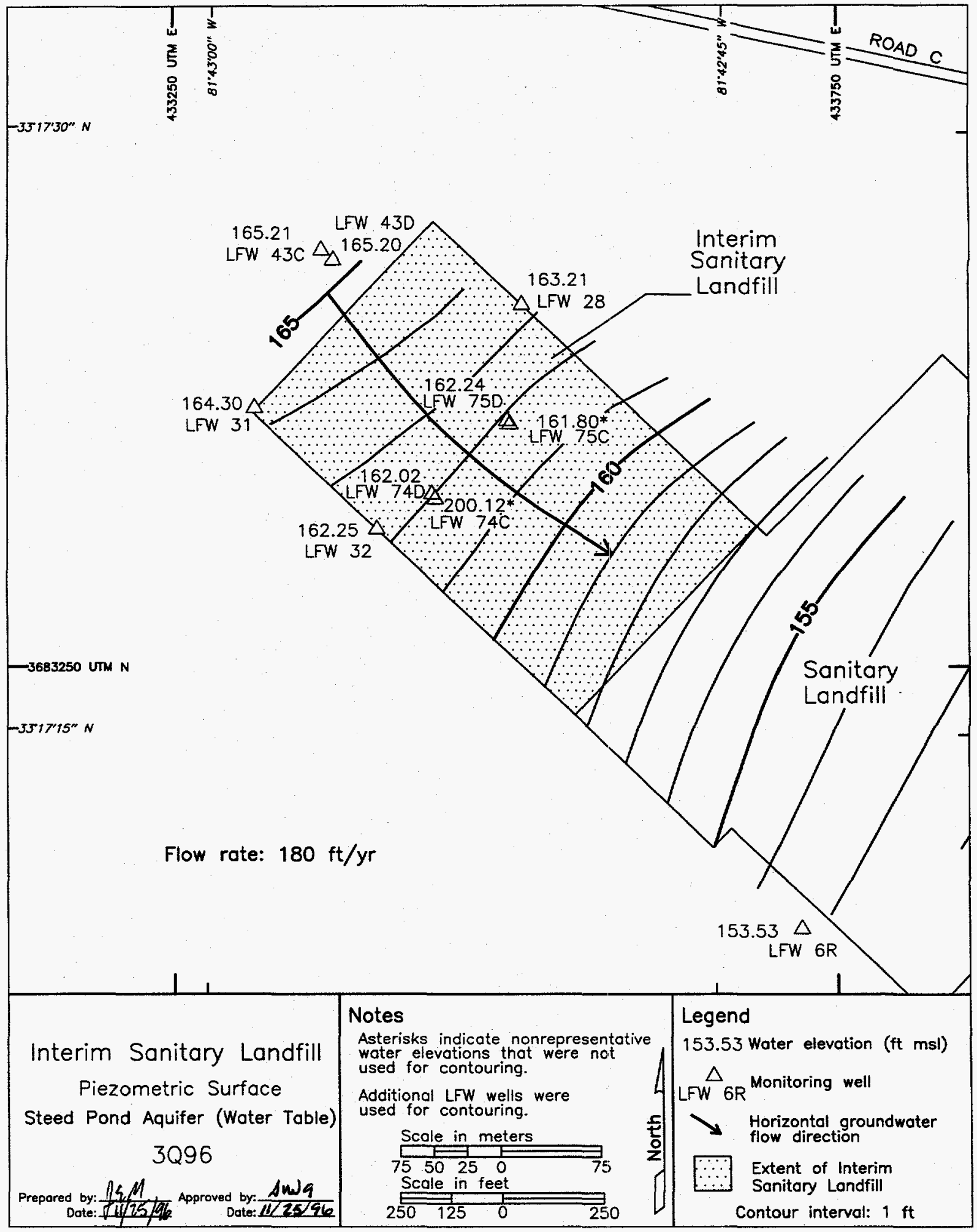

Figure 4. Piezometric Surface Map of Steed Pond Aquifer (Water Table) at the Interim Sanitary Landfill, Third Quarter 1996 


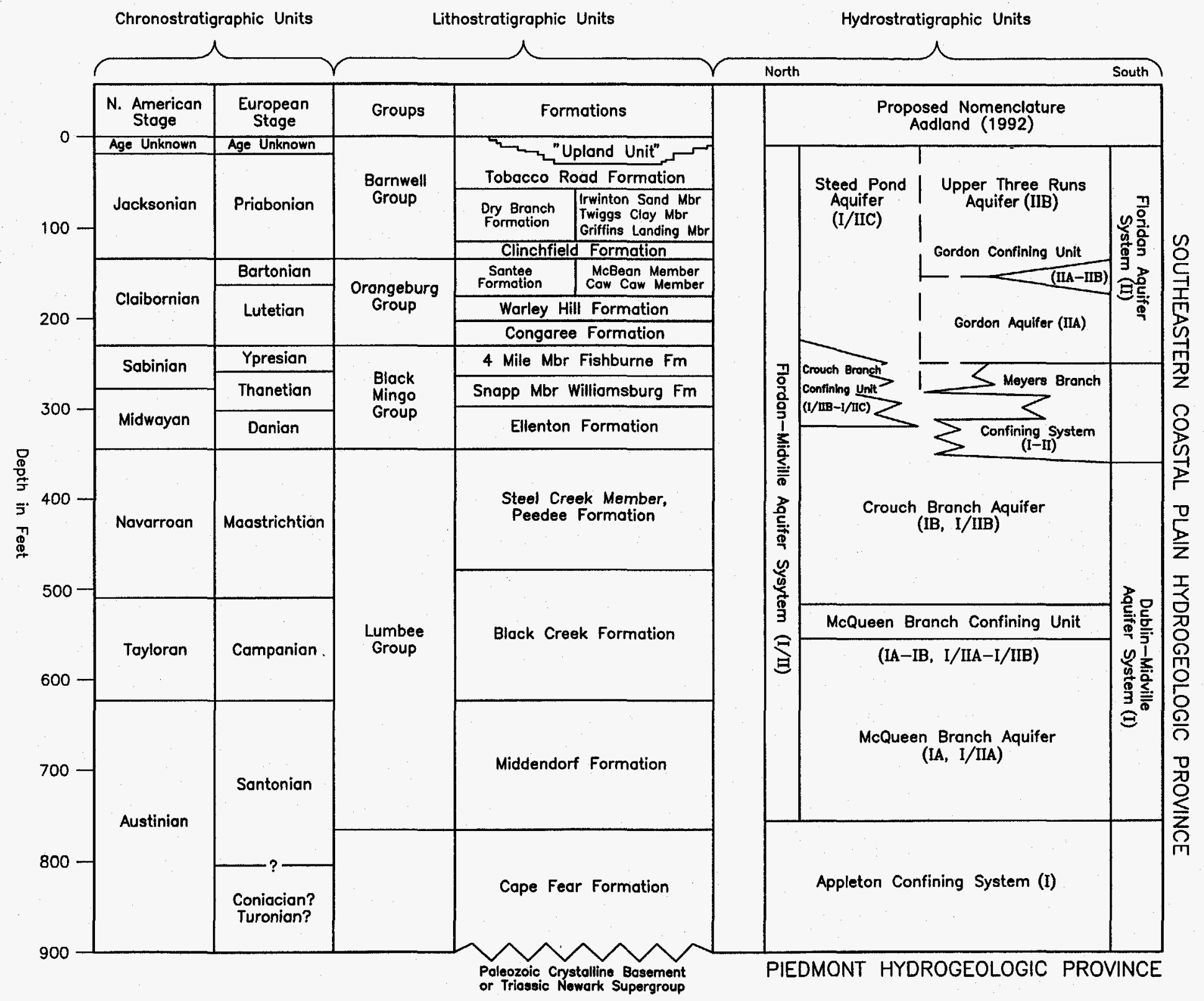

Figure 5. Regional Correlation of Hydrostratigraphic and Llthostratigraphic Nomenclature 
THIS PAGE LEFT BLANK INTENTIONALLY. 


\section{Appendix D}

Groundwater Monitoring Results Tables 
WSRC-TR-96-0186-4 Unclassified

THIS PAGE LEFT BLANK INTENTIONALLY. 


\section{Key to Reading the Tables}

The following abbreviations may appear in the data tables:

\section{Constituents}

Sp. conductance

specific conductance

\section{Laboratories}

CN

EM

GE or GP

SC

SP

TM

WA orWS

\section{Sampling Codes}

B

C

D

E

I

L

N

$P$

S

W

$x$

\section{Sampling Methods}

B

O

$P$

$S$

V

\section{Units}

$\mathrm{mg} / \mathrm{L}$

$\mathrm{msl}$

MSL

NTU

pCir
Clemson Technical Center, Inc.

Environmental Protection Department/Environmental Monitoring Section (EPD/EMS) Laboratory

General Engineering Laboratories, Inc.

Savannah River Technology Center

Spencer Testing Services, Inc.

Thermo NUtech

Roy F. Weston, Inc.

blank sample was collected

well was pumping continuously

well was dry

equipment blank was collected

well went dry during sampling; insufficient water to collect all samples

well went dry before sampling began; only depth to water can be determined

well was not stabilized before sampling began

inaccessibility or mechanical failure prevented sample collection and field analysis of the water

no water in standpipe; for water-level events only

purged water must be containerized; only water-level measurements were obtained

well went dry during purging; samples collected after well recovered

sample collected using an open-bucket bailer

sample collected by method other than bailer or pump

sample collected using a bladder pump

sample collected using a single-speed centrifugal downhole pump

sample collected using a variable-speed pump

milligrams per liter

mean sea level

million structures per liter

nephelometric turbidity unit

picocuries per liter 


\begin{tabular}{|c|c|}
\hline $\begin{array}{l}\mathrm{pCi} / \mathrm{mL} \\
\mathrm{pH} \\
\mu \mathrm{g} / \mathrm{L} \\
\mu \mathrm{S} / \mathrm{cm}\end{array}$ & $\begin{array}{l}\text { picocuries per milliliter } \\
\text { pH unit } \\
\text { micrograms per liter } \\
\text { microsiemens per centimeter }\end{array}$ \\
\hline \multicolumn{2}{|l|}{ Other } \\
\hline CS & carbon steel \\
\hline DF & dilution factor column in data tables \\
\hline$E$ & exponential notation (e.g., $1.1 \mathrm{E}-09=1.1 \times 10^{-9}=0.0000000011$ ) \\
\hline $\mathrm{H}$ & holding time column in data tables \\
\hline Mod & modifier column in data tables \\
\hline PDWS & Primary Drinking Water Standard \\
\hline PVC & polyvinyl chloride \\
\hline ST & exceeded standard column in data tables \\
\hline TOC & top of casing \\
\hline
\end{tabular}

\section{Results below Detection}

For radiological analyses, the analytical result field contains the result recorded on the analytical instrument and reported by the laboratory, even if it is negative. For nonradiological analyses, if the analyte is not detected, the sample-specific estimated quantitation limit (EQL) is entered into the result field and is reported with a less than [ $<]$ sign. The EQL is defined as the lowest concentration that can be achieved reliably within specified limits of precision and accuracy during routine laboratory operating conditions. The sample-specific EQL is modified for sample concentration or dilution or unusual aliquot size that affects analytical sensitivity.

\section{Holding Times}

Standard analytical methods include a limit, called holding time, on the maximum elapsed time between sample collection and extraction or analysis by the laboratory. In the data tables, a large bullet $(\bullet)$ in the $H$ (holding time) column indicates that holding time was exceeded. Analyses performed beyond holding times may not yield valid results.

The South Carolina Department of Health and Environmental Control (SCDHEC) allows only 15 minutes to elapse between sampling and analysis for $\mathrm{pH}$. Thus, only field $\mathrm{pH}$ measurements can meet the holding time criterion; laboratory $\mathrm{pH}$ analyses always will exceed it.

The laboratory procedure used for the determination of specific conductance allows one day to elapse between sampling and analysis. Thus, laboratory specific conductance measurements may exceed the holding time criterion.

\section{Data Rounding}

A constituent result in the analytical results tables that is not marked in the ST column but appears to equal an Appendix A standard is below the standard in the database. Values stored in the database contain more significant digits than the reported results. Apparent discrepancies in the tables are due to the rounding of reported results.

\section{Data Qualification}

The contract laboratories submit sample- or batch-specific quality assurance/quality control information either at the same time as analytical results or in a quarterly summary. Properly defined and used data modifiers (also referred to as qualifiers) can be a key component in 
assessing data usability. Modifiers designed by EPD/EMS and provided to the primary laboratories are defined below. These modifiers appear in the data tables under the column Mod. All lettered modifiers except $E$ are based on EPA's STORET codes.

\section{Modifiers}

(Blank)

$\mathrm{E}$

1

J

L

M

$\mathbf{R}$

$\mathrm{T}$

$U$

Y

Y

1

2

3
Data are not qualified. Numbers should be interpreted exactly as reported.

The detected result is between the sample-specific EQL and the method detection limit.

The value in the result field is the instrument reading, not the sample quantitation limit. Always used with the result qualifier $U$.

Value is estimated because quantitation in the sample or in associated quality control samples did not meet specifications.

Value is off-scale high. The actual value is not known but is known to be greater than the value shown.

Presence of the analyte is verified but not quantified.

Result was rejected because performance requirements in the sample analysis or associated quality control analyses were not met.

Analyte was not detected; if present, it was below the criteria for detection.

Material analyzed for but not detected. Analytical result reported is less than the sample quantitation limit.

Analyte was detected in an associated method blank.

Result was obtained from an unpreserved or improperly preserved sample. Data may not be accurate.

Result may be an underestimation of the true value due to analytical bias.

Result may be an overestimation of the true value due to analytical bias.

The associated result may be of poor precision (high variability) due to analytical bias.

Note: These are only some of the qualifiers present in the database. All modifiers associated with the data are published in the result tables of EPD/EMS' quarterly groundwater monitoring reports, the official repository of the data. 
Table D-1. Maximum Levels of Constituents Exceeding the Practical Quantitation Limits or Tolerance Limits

\begin{tabular}{|c|c|c|c|c|c|c|}
\hline Well & Constituent & Unit & 1096 & Mod & 3096 & Mod \\
\hline \multicolumn{7}{|c|}{ Upper Steed Pond } \\
\hline LFW 28 & $\begin{array}{l}\text { Barium } \\
\text { Copper } \\
\text { Specific conductance }\end{array}$ & $\begin{array}{l}\mu g / L \\
\mu g / L \\
\mu S / c m\end{array}$ & $\begin{array}{l}31 \\
37 \\
50\end{array}$ & $\begin{array}{l}V \\
V\end{array}$ & $\begin{array}{l}26 \\
\overline{1.9}\end{array}$ & \\
\hline LFW 32 & $\begin{array}{l}\text { Dichloromethane } \\
1,1,1 \text {-Trichloroethane } \\
\text { Trichlorofluoromethane }\end{array}$ & $\begin{array}{l}\mu g / L \\
\mu g / L \\
\mu g / L\end{array}$ & $\begin{array}{l}- \\
7.9 \\
96\end{array}$ & V & $\begin{array}{l}37 \\
13 \\
170\end{array}$ & V \\
\hline LFW 74D & $\begin{array}{l}\text { 1,1,1-Trichloroethane } \\
\text { Trichlorofluoromethane } \\
\text { Zinc }\end{array}$ & $\begin{array}{l}\mu g / L \\
\mu g / L \\
\mu g / L\end{array}$ & $\begin{array}{l}- \\
15 \\
54\end{array}$ & $J$ & $\begin{array}{l}6.4 \\
39 \\
-\end{array}$ & J1 \\
\hline LFW 75D & Zinc & $\mu g / L$ & 43 & v & - & \\
\hline \multicolumn{7}{|c|}{ Middle Steed Pond } \\
\hline LFW 43C & Specific conductance & $\mu \mathrm{S} / \mathrm{cm}$ & 170 & & - & \\
\hline LFW 75C & Zinc & $\mu \mathrm{g} / \mathrm{L}$ & 32 & v & - & \\
\hline
\end{tabular}

$-=$ analyzed but not above standards.

Notes: This table presents the highest values for duplicate/replicate results. The groundwater samples are unfiltered; therefore, the results for metals are for total recoverable metals. 
Table D-2. Constituents Exceeding the SRS Flag 2 Criteria

\begin{tabular}{|c|c|c|c|c|c|c|}
\hline Well & Constituent & Unit & 1096 & Mod & 3096 & Mod \\
\hline \multicolumn{7}{|c|}{ Upper Steed Pond } \\
\hline LFW 28 & Dichloromethane & $\mu g / L$ & - & & 5.1 & V \\
\hline LFW 32 & $\begin{array}{l}\text { Dichloromethane } \\
\text { Thallium } \\
\text { Trichlorofluoromethane }\end{array}$ & $\begin{array}{l}\mu g / L \\
\mu g / L \\
\mu g / L\end{array}$ & $\begin{array}{l}\overline{-} \\
96\end{array}$ & $v$ & $\begin{array}{l}37 \\
2.4 \\
170\end{array}$ & $\begin{array}{l}V \\
J\end{array}$ \\
\hline LFW 74D & Trichlorofluoromethane & $\mu g / L$ & 15 & & 39 & J1 \\
\hline LFW 75D & Antimony & $\mu g / L$ & - & & 6.1 & \\
\hline \multicolumn{7}{|c|}{ Middle Steed Pond } \\
\hline LFW $75 \mathrm{C}$ & Thallium & $\mu g / L$ & 2.9 & $\mathrm{~J}$ & $=$ & $v$ \\
\hline
\end{tabular}

$-=$ analyzed but not above the Flag 2 criterion.

Notes: This table presents the highest values for duplicate/replicate results. The groundwater samples are unfiltered; therefore, the results for metals are for total recoverable metals. Flags are established by EPD/EMS and are based on final PDWS, Secondary Drinking Water Standards, or method detection limits (see Appendix B). 
Table D-3. Groundwater Monitoring Results for Individual Wells

WELL LFW 28

\begin{tabular}{|c|c|c|c|c|c|c|}
\hline SRS Coord. & Lat/Longitude & Screen Zone Elevation & Top of Casing & Casing & Pump & Screen Zone \\
\hline $\begin{array}{l}\text { N86079.6 } \\
\text { E45555.3 }\end{array}$ & $\begin{array}{l}33.290475^{\circ} \mathrm{N} \\
81.714056^{\circ} \mathrm{W}\end{array}$ & $162.1-141.1 \mathrm{ft} \mathrm{msl}$ & $192.4 \mathrm{ft} \mathrm{msl}$ & 4" PVC & $s$ & Upper Steed Pond \\
\hline SAMPLE DATE & & & & & & \\
\hline
\end{tabular}

FIELD DATA

\begin{tabular}{ll} 
Parameter & 1096 \\
\hline Water elevation & 163.1 \\
pH & 5.2 \\
Sp. conductance & 46 \\
Water temperature & 24.0 \\
Alkalinity as $\mathrm{CaCO}_{3}$ & 6 \\
Turbidity & 8 \\
Volume purged & 0.069 \\
Sampling code & $\mathrm{XN}$
\end{tabular}

3096
163.2
5.2
38
21.6
5
1
2.1

Unit

tt msl $\mathrm{pH}$ $\mu \mathrm{S} / \mathrm{cm}$ ${ }^{\circ} \mathrm{C}$ $\mathrm{mgh}$ NTU well vol

ANALYTICAL DATA

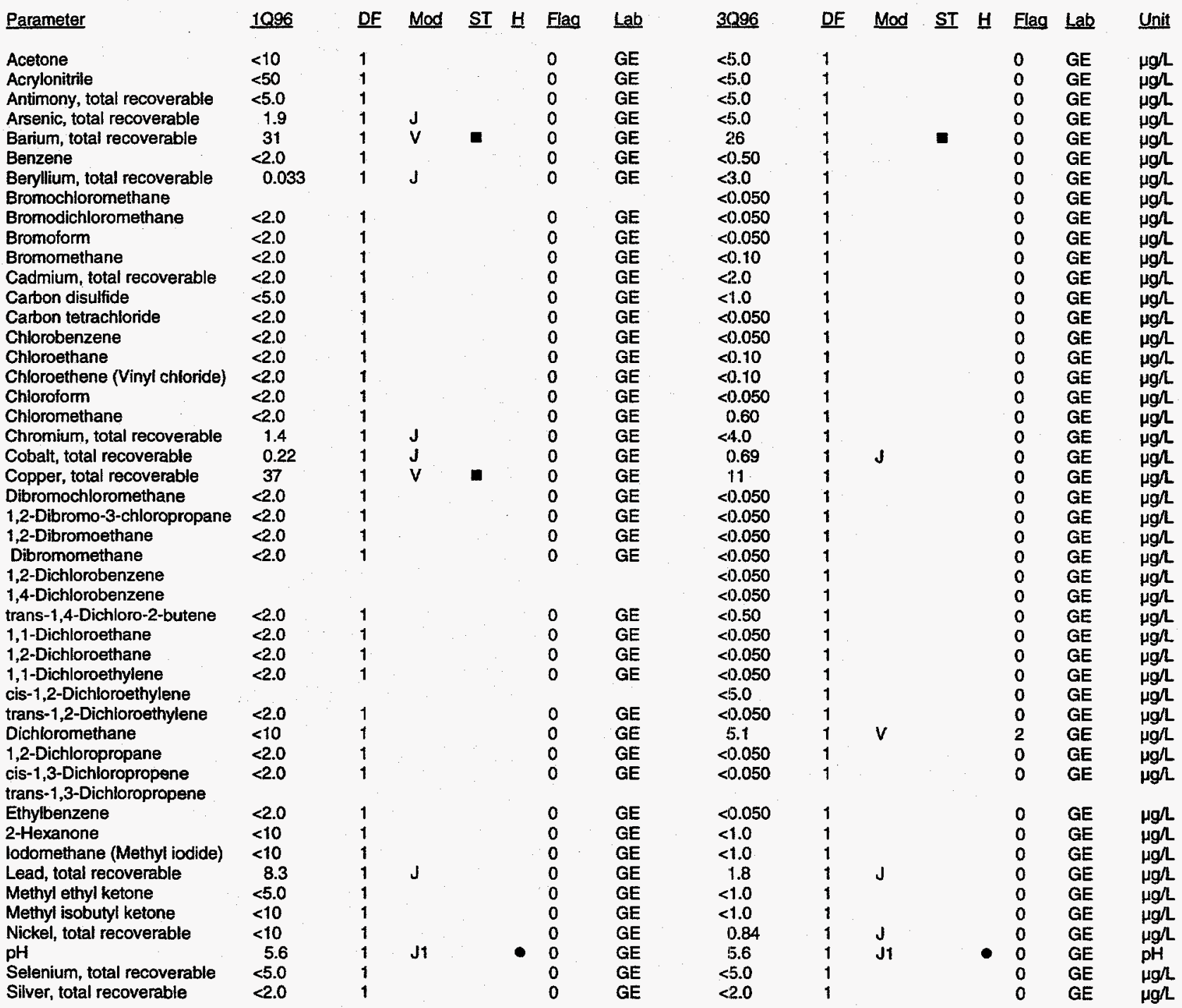

- = exceeded holding time.

$\square=$ exceeded standard. 
WELL LFW 28 (cont.)

Parameter

Specific conductance

Styrene

$1,1,1,2$-Tetrachloroethane

1,1,2,2-Tetrachloroethane

Tetrachloroethylene

Thallium, total recoverable

Toluene

1,1,1-Trichloroethane

1,1,2-Trichloroethane

Trichloroethylene

Trichlorofluoromethane

1,2,3-Trichloropropane

Vanadium, total recoverable

Vinyl acetate

Xylenes

Zinc, total recoverable
브 Flag Lab

n

GE
GE
$G E$
$G E$
$G E$
$G E$
$G E$
$G E$
$G E$
$G E$
$G E$
$G E$
$G E$
$G E$
$G E$
$G E$

3Q96

1.9

$<0.050$

$<0.050$

$<0.050$

$<0.050$

$<5.0$

0.18

$<0.050$

$<0.050$

$<0.050$

$<0.50$

$<0.050$

0.52

$<1.0$

$<0.15$

13
DF Mad

ST 브 Flag Lab

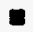

$\begin{array}{ll}0 & \mathrm{GE} \\ 0 & \mathrm{GE} \\ 0 & \mathrm{GE} \\ 0 & \mathrm{GE} \\ 0 & \mathrm{GE} \\ 0 & \mathrm{GE} \\ 0 & \mathrm{GE} \\ 0 & \mathrm{GE} \\ 0 & \mathrm{GE} \\ 0 & \mathrm{GE} \\ 0 & \mathrm{GE} \\ 0 & \mathrm{GE} \\ 0 & \mathrm{GE} \\ 0 & \mathrm{GE} \\ 0 & \mathrm{GE} \\ 0 & \mathrm{GE}\end{array}$

Unit

$\mu S / \mathrm{cm}$

$\mu g /$

$\mu \mathrm{g} / \mathrm{L}$

$\mu g /$ L

$\mu \mathrm{gh}$

$\mu g /$ L

$\mu$ gh

$\mu \mathrm{g} / \mathrm{L}$

$\mu g$ L

$\mu g h$

$\mu g /$

$\mu g /$

$\mathrm{ug} / \mathrm{L}$

$\mu g / L$

$\mu g / L$

- exceeded holding time.

- exceeded standard. 
WELL LFW 32

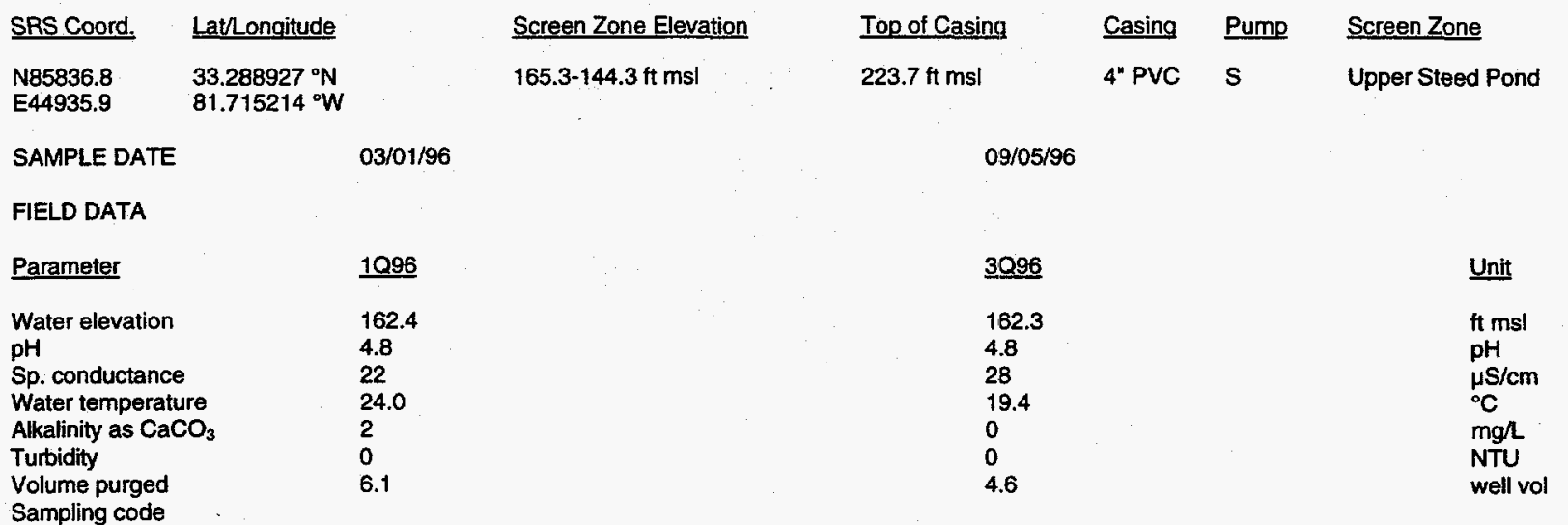

ANALYTICAL DATA

\begin{tabular}{|c|c|c|c|c|c|c|c|c|c|c|c|c|c|c|c|}
\hline Parameter & 1096 & DF & $\underline{\text { Mod }}$ & $\underline{\mathbf{S T}}$ & H & Flag & $\underline{\text { Lab }}$ & $\underline{3096}$ & $\underline{\mathrm{DF}}$ & Mod & $\underline{\text { ST }}$ & 브 & Flag & $\underline{\text { Lab }}$ & Unit \\
\hline Acetone & $<10$ & 1 & & & & 0 & GE & $<10$ & 1 & & & & 0 & WA & $\mu g /$ \\
\hline Acrylonitrile & $<50$ & 1 & & & & 0 & GE & $<5.0$ & 1 & & & & 0 & GE & $\mu g h$ \\
\hline Antimony, total recoverable & 1.7 & 1 & $\mathbf{J}$ & & & 0 & GE & $<5.0$ & 1 & & & & 0 & GE & $\mu g / L$ \\
\hline Arsenic, total recoverable & $<5.0$ & 1 & & & & 0 & GE & $<5.0$ & 1 & & & & 0 & GE & $\mu g h$ \\
\hline Barium, total recoverable & 7.8 & 1 & $\mathrm{v}$ & & & 0 & GE & 12 & 1 & & & & 0 & WA & $\mu g / L$ \\
\hline Benzene & $<2.0$ & 1 & & & & 0 & GE & 0.060 & 1 & $\mathrm{~J}$ & & & 0 & GE & $\mu g / L$ \\
\hline $\begin{array}{l}\text { Beryllium, total recoverable } \\
\text { Bromochloromethane }\end{array}$ & $<3.0$ & 1 & & & & 0 & GE & $\begin{array}{l}<1.6 \\
<0.050\end{array}$ & 1 & & & & 0 & WA & $\mu g / 2$ \\
\hline Bromodichloromethane & $<2.0$ & 1 & & & & 0 & GE & $<0.050$ & $i$ & & & & $\begin{array}{l}0 \\
0\end{array}$ & $\begin{array}{l}\text { GE } \\
\text { GE }\end{array}$ & $\begin{array}{l}\mu g / L \\
\mu g / L\end{array}$ \\
\hline Bromoform & $<2.0$ & 1 & & & & 0 & $\mathrm{GE}$ & $<0.050$ & 1 & & & & 0 & $\mathrm{GE}$ & $\mu g / L$ \\
\hline Bromomethane & $<2.0$ & 1 & & & & 0 & GE & $<0.10$ & 1 & & & & 0 & GE & $\mu g / L$ \\
\hline Cadmium, total recoverable & $<2.0$ & 1 & & & & 0 & GE & $<2.0$ & 1 & & & & 0 & GE & $\mu g / 2$ \\
\hline Carbon disulfide & $<5.0$ & 1 & & & & 0 & GE & $<1.0$ & 1 & & & & 0 & $\mathrm{GE}$ & $\mu g \Omega$ \\
\hline Carbon tetrachloride & $<2.0$ & 1 & & & & 0 & $\mathrm{GE}$ & $<0.050$ & 1 & & & & 0 & GE & $\mu g /$ \\
\hline Chlorobenzene & $<2.0$ & 1 & & & & 0 & GE & $<0.050$ & 1 & & & & 0 & $\overline{G E}$ & $\mu g / 2$ \\
\hline Chloroethane & $<2.0$ & 1 & & & & 0 & GE & $<0.10$ & 1 & & & & 0 & GE & $\mu g h$ \\
\hline Chloroethene (Vinyl chloride) & $<2.0$ & 1 & & & & 0 & GE & $<0.10$ & 1 & & & & 0 & GE & $\mu g / 2$ \\
\hline Chloroform & $<2.0$ & 1 & & & & 0 & GE & 0.17 & 1 & & & & 0 & GE & $\mu g \Omega$ \\
\hline Chloromethane & $<2.0$ & 1 & & & & 0 & GE & $<0.10$ & 1 & & & & 0 & GE & $\mu g / L$ \\
\hline Chromium, total recoverabie & 0.63 & 1 & $J$ & & & 0 & GE & 0.67 & 1 & $J$ & & & 0 & GE & $\mu g / \mathrm{L}$ \\
\hline Cobalt, total recoverable & $<4.0$ & 1 & & & & 0 & GE & 0.86 & 1 & $J$ & & & 0 & GE & $\mu g \mathrm{~L}$ \\
\hline Copper, total recoverable & $<4.7$ & 1 & v & & & 0 & GE & 13 & 1 & $\mathbf{J}$ & & & $\mathbf{0}$ & WA & $\mu g /$ \\
\hline Dibromochloromethane & $<2.0$ & 1 & & & & 0 & GE & $<0.050$ & 1 & & & & 0 & GE & $\mu g \Omega$ \\
\hline 1,2-Dibromo-3-chloropropane & $<2.0$ & 1 & & & & 0 & GE & $<0.050$ & 1 & & & & 0 & $\overline{G E}$ & $\mu g / \mathrm{L}$ \\
\hline 1,2-Dibromoethane & $<2.0$ & 1 & & & & 0 & GE & $<0.050$ & 1 & & & & 0 & GE & $\mu g /$. \\
\hline Dibromomethane & $<2.0$ & 1 & & & & 0 & GE & $<0.050$ & 1 & & & & 0 & GE & $\mu g /$ \\
\hline 1,2-Dichlorobenzene & & & & & & & & $<0.050$ & 1 & & & & 0 & GE & $\mu g \Omega$ \\
\hline 1,4-Dichlorobenzene & & & & & & & & $<0.050$ & 1 & & & & 0 & GE & $\mu g /$ \\
\hline trans-1,4-Dichloro-2-butene & $<2.0$ & 1 & & & & 0 & GE & $<0.50$ & 1 & & & & 0 & GE & $\mu g / L$ \\
\hline 1,1-Dichloroethane & $<2.0$ & 1 & & & & 0 & GE & 1.6 & 1 & & & & 0 & GE & $\mu g / L$ \\
\hline 1,2-Dichloroethane & $<2.0$ & 1 & & & & 0 & GE & $<0.050$ & 1 & & & & 0 & GE & $\mu g / L$ \\
\hline 1,1-Dichloroethylene & $<2.0$ & 1 & & & & 0 & GE & 0.65 & 1 & & & & 0 & GE & $\mu g / L$ \\
\hline cis-1,2-Dichloroethylene & & & & & & & & $<5.0$ & 1 & & & & 0 & WA & $\mu g /$ \\
\hline trans-1,2-Dichloroethylene & $<2.0$ & 1 & & & & 0 & GE & $<0.050$ & 1 & & & & 0 & GE & $\mu g / L$ \\
\hline Dichloromethane & $<10$ & 1 & & & & 0 & GE & 37 & 10 & $v$ & = & & 2 & $\mathrm{GE}$ & $\mu g h$ \\
\hline 1,2-Dichloropropane & $<2.0$ & 1 & & & & 0 & GE & $<0.050$ & 1 & & & & 0 & GE & $\mu g / L$ \\
\hline cis-1,3-Dichloropropene & $<2.0$ & $i$ & & & & 0 & GE & $<0.050$ & $i$ & & & & 0 & $\mathrm{GE}$ & $\mu g / L$ \\
\hline trans-1,3-Dichloropropene & $<2.0$ & 1 & & & & 0 & GE & & & & & & & & $\mu g / L$ \\
\hline Ethylbenzene & $<2.0$ & 1 & & & & 0 & GE & $<0.050$ & 1 & & & & 0 & GE & $\mu g$ L \\
\hline 2-Hexanone & $<10$ & 1 & & & & 0 & GE & $\ll 1.0$ & 1 & & & & 0 & GE & $\mu g / L$ \\
\hline lodomethane (Methyl iodide) & $<10$ & 1 & & & & 0 & GE & $<1.0$ & 1 & & & & 0 & GE & $\mu g /$ \\
\hline Lead, total recoverable & $<5.0$ & 1 & $\mathbf{J}$ & & & 0 & GE & $<5.0$ & 1 & & & & 0 & GE & $\mu g /$ \\
\hline Methyl ethyl ketone & $<5.0$ & 1 & & & & 0 & GE & $<0.38$ & 1 & $v$ & & & 0 & GE & $\mu g / L$ \\
\hline Methyl isobutyl ketone & $<10$ & 1 & & & & 0 & GE & $<1.0$ & 1 & & & & 0 & GE & $\mu g / L$ \\
\hline Nickel, total recoverable & $<10$ & 1 & & & & 0 & GE & $<10$ & 1 & & & & 0 & GE & $\mu g / \mathrm{L}$ \\
\hline & 5.3 & 1 & J1 & & - & 0 & GE & 5.1 & 1 & J1 & & - & 0 & $\mathrm{GE}$ & pH \\
\hline Selenium, total recoverable & $<5.0$ & 1 & & & & 0 & GE & $<5.0$ & 1 & & & & 0 & $\mathrm{GE}$ & $\mu g / L$ \\
\hline Silver, total recoverable & $<2.0$ & 1 & & & & 0 & GE & $<0.90$ & 1 & v & & & 0 & WA & $\mu g \Omega$ \\
\hline Specific conductance & 24 & 1 & & & & 0 & GE & 31 & 1 & & & & 0 & GE & $\mu \mathrm{S} / \mathrm{cm}$ \\
\hline Styrene & $<2.0$ & 1 & & & & 0 & GE & $<0.050$ & 1 & & & & 0 & GE & $\mu g / L$ \\
\hline $1,1,1,2-$ Tetrachloroethane & $<2.0$ & $t$ & & & & 0 & GE & $<0.050$ & 1 & & & & 0 & GE & $\mu g h$ \\
\hline
\end{tabular}

- = exceeded holding time.

= exceeded standard. 
WELL LFW 32 (cont.)

Parameter

1,1,2,2-Tetrachloroethane Tetrachloroethylene

Thallium, total recoverable

Toluene

1,1,1-Trichloroethane

1,1,2-Trichloroethane

Trichloroethylene

Trichlorofluoromethane

1,2,3-Trichloropropane

Vanadium, total recoverable

Vinyl acetate

Xylenies

Zinc, total recoverable
1096

$<2.0$

$<2.0$

$<5.0$

$<2.0$

$<2.0$

20

$<2.0$

$<2.0$
55

$<2.0$

$<10$

$<10$
$<10$

$<2.0$

$<6.0$
DF Mod ST $\underline{H}$ Flag Lab

GE $\quad<0.050$

GE $\quad 0.36$

GE

GE

GE

GE

GE

GE

GE

GE

V $\underline{3096}$

2.4

1.6

13

$<0.050$

0.63

170

$<0.050$

0.86

$<1.0$

$<0.15$

23
DF Mod ST $\underline{\text { H Flag Lab Unit }}$

GE $\mu g h$

$\mu \mathrm{g} / \mathrm{L}$

$\mu g / 2$

$\mu g /$.

$\mu g h$

$\mu g / L$

$\mu g / 2$

$\mu \mathrm{g} / \mathrm{L}$

$\mu g h$.

$\mu g / L$

$\mu g /$

$\mu g h$

$\mu g / L$

- exceeded holding time.

a exceeded standard. 
WELL LFW 43C

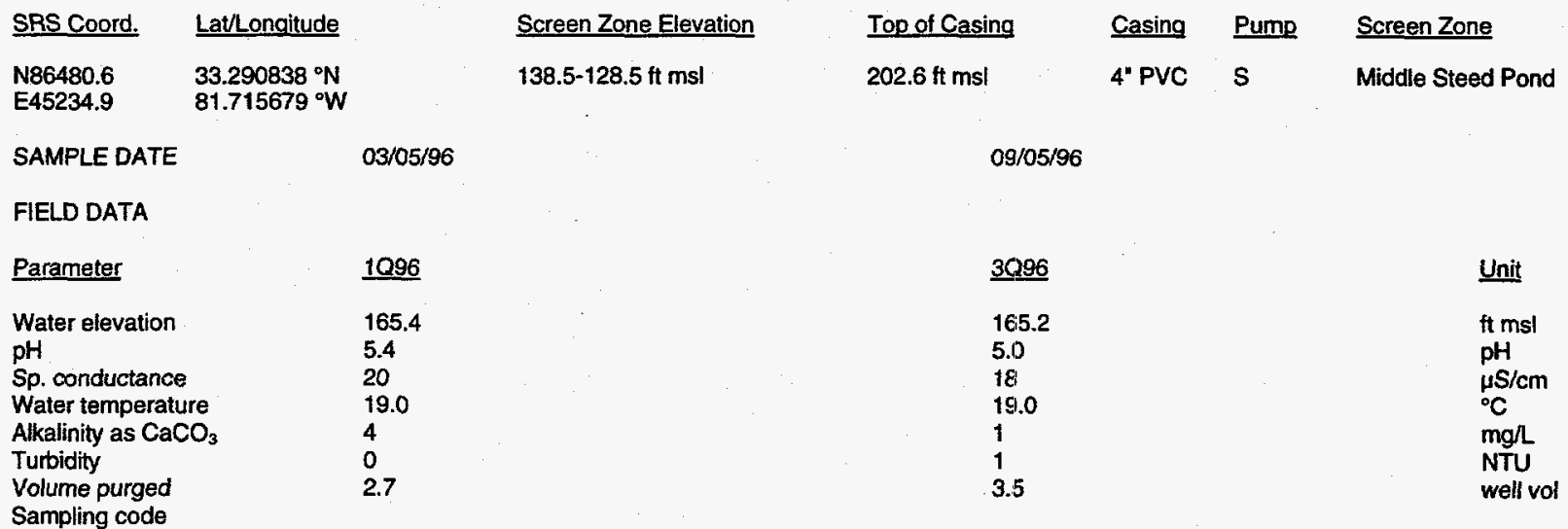

ANALYTICAL DATA

\begin{tabular}{|c|c|c|c|c|c|c|c|c|c|c|c|c|c|c|c|}
\hline Parameter & 1096 & DF & Mod & ST & $\underline{\text { H}}$ & Flag & $\underline{\underline{L a b}}$ & $\underline{3096}$ & DF & Mod & $\underline{S T}$ & H & Flag & Lab & Unit \\
\hline Acetone & $<2.7$ & 1 & V & & & 0 & WA & $<3.7$ & 1 & JV1 & & - & 0 & GE & $\mu g /$ \\
\hline Acrylonitrile & $<10$ & 1 & & & & 0 & WA & $<5.0$ & 1 & $\$ 1$ & & $\cdot$ & 0 & GE & $\mu g^{\prime} L$ \\
\hline Antimony, total recoverable & $<0.20$ & 1 & & & & 0 & GE & $<5.0$ & 1 & & & & 0 & GE & $\mu g / L$ \\
\hline Arsenic, total recoverable & $<3.0$ & 1 & $J$ & & & 0 & GE & $<5.0$ & 1 & & & & 0 & GE & $\mu g / L$ \\
\hline Barium, total recoverable & 4.8 & 1 & JV & & & 0 & $\overline{G E}$ & 5.8 & 1 & & & & 0 & GE & $\mu g / L$ \\
\hline Benzene & $<2.0$ & 1 & $\mathbf{J}$ & & & 0 & GE & $<0.50$ & 1 & J1 & & $\bullet$ & 0 & $\overline{G E}$ & $\mu g /$ \\
\hline Berylifium, total recoverable & 0.090 & 1 & $J$ & & & 0 & GE & $<3.0$ & 1 & & & & 0 & GE & $\mu g / L$ \\
\hline Bromochloromethane & $<5.0$ & 1 & & & & 0 & WA & $<0.050$ & 1 & J1 & & - & 0 & GE & $\mu g / L$ \\
\hline Bromodichloromethane & $<2.0$ & 1 & & & & 0 & GE & $<0.050$ & 1 & J1 & & 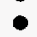 & 0 & $\mathrm{GE}$ & $\mu g / L$ \\
\hline Bromoform & $<2.0$ & 1 & & & & 0 & GE & $<0.050$ & 1 & J1 & & 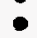 & 0 & GE & $\mu g h$. \\
\hline Bromomethane & $<2.0$ & 1 & & & & 0 & GE & $<0.10$ & $i$ & Ji & & 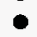 & 0 & GE & $\mu g / L$ \\
\hline Cadmium, total recoverable & $<0.40$ & 1 & & & & 0 & $\mathrm{GE}$ & $<2.0$ & 1 & & & & 0 & GE & $\mu g / L$ \\
\hline Carbon disulfide & 1.1 & 1 & $J$ & & & 0 & WA & $<1.0$ & 1 & J1 & & $\bullet$ & 0 & $\mathrm{GE}$ & $\mu g / \mathrm{L}$ \\
\hline Carbon tetrachloride & $<2.0$ & 1 & & & & 0 & $\mathrm{GE}$ & $<0.050$ & 1 & j1 & & $e$ & 0 & GE & $\mu g / L$ \\
\hline Chlorobenzene & $<2.0$ & 1 & & & & 0 & GE & $<0.050$ & 1 & J1 & & $\bullet$ & 0 & $\overline{\mathrm{GE}}$ & $\mu g / L$ \\
\hline Chloroethane & $<2.0$ & 1 & & & & 0 & $\overline{\mathrm{GE}}$ & $<0.10$ & 1 & J1 & & $\bullet$ & 0 & GE & $\mu g /$ \\
\hline Chloroethene (Vinyl chloride) & $<2.0$ & 1 & & & & 0 & GE & $<0.10$ & 1 & J1 & & 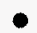 & 0 & $\overline{\mathrm{GE}}$ & $\mu g / L$ \\
\hline Chloroform & $<2.0$ & 1 & & & & 0 & GE & $<0.050$ & 1 & J1 & & 0 & 0 & GE & $\mu g /$ \\
\hline Chloromethane & $<2.0$ & 1 & & & & 0 & GE & $<0.10$ & 1 & ji & & $\bullet$ & 0 & GE & $\mu g /$ \\
\hline Chromium, total recoverable & 0.79 & 1 & J & & & 0 & $\mathrm{GE}$ & 0.60 & 1 & $j$ & & & 0 & GE & $\mu g / L$ \\
\hline Cobalt, total recoverable & $<4.5$ & 1 & & & & 0 & WA & 0.47 & 1 & $\mathrm{~J}$ & & & 0 & GE & $\mu g /$ \\
\hline Copper, total recoverable & 4.5 & 1 & & & & 0 & GE & 1.7 & 1 & $\mathbf{J}$ & & & 0 & $\mathrm{GE}$ & $\mu g /$ \\
\hline Dibromochloromethane & $<2.0$ & 1 & & & & 0 & GE & $<0.050$ & 1 & $J 1$ & & $\bullet$ & 0 & $\mathrm{GE}$ & $\mu g / \mathrm{L}$ \\
\hline 1,2-Dibromo-3-chloropropane & $<2.0$ & 1 & & & & 0 & $\overline{\mathrm{GE}}$ & $<0.050$ & 1 & J1 & & 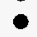 & 0 & GE & $\mu g / 2$ \\
\hline 1,2 -Dibromoethane & $<2.0$ & 1 & & & & 0 & GE & $<0.050$ & 1 & J1 & & - & 0 & GE & $\mu g / L$ \\
\hline Dibromomethane & $<2.0$ & 1 & & & & 0 & GE & $<0.050$ & 1 & J1 & & 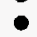 & 0 & GE & $\mu g / L$ \\
\hline 1,2-Dichlorobenzene & $<5.0$ & 1. & & & & 0 & WA & $<0.050$ & 1 & J1 & & - & 0 & GE & $\mu g / L$ \\
\hline 1,4-Dichlorobenzene & $<5.0$ & 1 & & & & 0 & WA & $<0.050$ & 1 & J1 & & 6 & 0 & GE & $\mu g / 2$ \\
\hline trans-1,4-Dichloro-2-butene & $<2.0$ & 1 & & & & 0 & GE & $<0.50$ & 1 & J1 & & - & 0 & $\overline{G E}$ & $\mu \mathrm{g} / \mathrm{L}$ \\
\hline 1,1-Dichloroethane & $<2.0$ & 1 & & & & 0 & GE & $<0.050$ & 1 & Jt & & - & 0 & GE & $\mu g / L$ \\
\hline 1,2-Dichloroethane & $<2.0$ & 1 & & & & 0 & GE & $<0.050$ & 1 & J1 & & 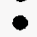 & 0 & GE & $\mu g / L$ \\
\hline 1,1-Dichloroethylene & $<2.0$ & 1 & & & & 0 & GE & $<0.050$ & 1 & J1 & & 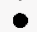 & 0 & GE & $\mu g / L$ \\
\hline cis-1,2-Dichloroethylene & $<5.0$ & 1 & & & & 0 & WA & $<5.0$ & $i$ & J1 & & $\bullet$ & 0 & GE & $\mu g / L$ \\
\hline trans-1,2-Dichloroethylene & $<2.0$ & 1 & & & & 0 & GE & $<0.050$ & 1 & J1 & & - & 0 & $\overline{G E}$ & $\mu \mathrm{g} / \mathrm{L}$ \\
\hline Dichloromethane & $<1.0$ & 1 & V & & & 0 & $\overline{G E}$ & $<4.0$ & 1 & JV1 & & - & 0 & GE & $\mu g / L$ \\
\hline 1,2-Dichloropropane & $<2.0$ & 1 & & & & 0 & $\mathrm{GE}$ & $<0.050$ & $i$ & J1 & & - & 0 & GE & $\mu g / L$ \\
\hline $\begin{array}{l}\text { cis-1,3-Dichloropropene } \\
\text { trans-1,3-Dichloropropene }\end{array}$ & $<2.0$ & 1 & & & & 0 & $\mathrm{GE}$ & $<0.050$ & $i$ & J1 & & $\bullet$ & 0 & $\mathrm{GE}$ & $\mu g /$ \\
\hline Ethylbenzene & $<2.0$ & 1 & & & & 0 & GE & $<0.050$ & 1 & J1 & & - & 0 & GE & $\mu g / L$ \\
\hline 2-Hexanone & $<10$ & 1 & & & & 0 & $\overline{G E}$ & $<1.0$ & 1 & Ji & & 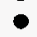 & 0 & GE & $\mu g / L$ \\
\hline lodomethane (Methyl iodide) & $<5.0$ & 1 & & & & 0 & WA & $<1.0$ & 1 & Ji & & - & 0 & GE & $\mu g / L$ \\
\hline Lead, total recoverable & 2.5 & 1 & & & & 0 & GE & 2.8 & $i$ & $J$ & & & 0 & GE & $\mu g / L$ \\
\hline Methyl ethyl ketone & $<5.0$ & 1 & & & & 0 & GE & $<1.0$ & $i$ & J1 & & $\bullet$ & 0 & GE & $\mu g / L$ \\
\hline Methyl isobutyl ketone & $<10$ & 1 & & & & 0 & GE & $<1.0$ & 1 & $J 1$ & & 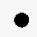 & 0 & $\overline{G E}$ & $\mu g / L$ \\
\hline Nickel, total recoverable & 0.36 & 1 & $\mathbf{J}$ & & & 0 & $\mathrm{GE}$ & $<10$ & 1 & & & & 0 & GE & $\mu g / L$ \\
\hline $\mathrm{pH}$ & 5.4 & 1 & J1 & & $\bullet$ & 0 & GE & 5.4 & 1 & J1 & & $\bullet$ & 0 & $\overline{\mathrm{GE}}$ & $\mathrm{pH}$ \\
\hline Selenium, total recoverable & $<2.0$ & 1 & & & & 0 & GE & $<5.0$ & 1 & & & & 0 & $\overline{G E}$ & $\mu g / L$ \\
\hline Silver, total recoverable & 0.076 & 1 & $J$ & & & 0 & $\overrightarrow{G E}$ & $<2.0$ & 1 & & & & 0 & GE & $\mu g /$ \\
\hline Specific conductance & 170 & 1 & & $\mathbf{\square}$ & & 0 & WA & 18 & 1 & & & & 0 & GE & $\mu S / \mathrm{cn}$ \\
\hline Styrene & $<2.0$ & 1 & & & & 0 & GE & $<0.050$ & 1 & J1 & & ? & 0 & GE & $\mu g /$ \\
\hline $1,1,1,2-$ Tetrachloroethane & $<2.0$ & 1 & & & & 0 & $\mathrm{GE}$ & $<0.050$ & 1 & Jt & & 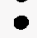 & 0 & GE & $\mu g / L$ \\
\hline
\end{tabular}

= exceeded holding time.

- = exceeded standard. 
WELL LFW 43C (cont.)

\section{Parameter}

1,1,2,2-Tetrachloroethane

Tetrachloroethylene

Thallium, total recoverable

Toluene

1,1,1-Trichloroethane

1,1,2-Trichloroethane

Trichloroethylene

Trichlorofluoromethane

1,2,3-Trichloropropane

vanadium, total recoverable

Vinyl acetate

Xylenes

Zinc, total recoverable
1096

$<2.0$

$<2.0$

0.029

$<2.0$

$<2.0$

$<2.0$

$<2.0$

$<2.0$

$<2.0$

$<6.9$

$<10$

$<2.0$

6.6
DF Mod ST H Flag Lab

$\mathrm{GE}$
$\mathrm{GE}$
$\mathrm{GE}$
$\mathrm{GE}$
$\mathrm{GE}$
$\mathrm{GE}$
$\mathrm{GE}$
$\mathrm{GE}$
$\mathrm{GE}$
$\mathrm{WA}$
$\mathrm{GE}$
$\mathrm{GE}$
$\mathrm{GE}$
3096

$<0.050$

$<0.050$

$<5.0$

$<0.19$

$<0.050$

$<0.050$

$<0.050$

$<0.50$

$<0.050$

0.71

$<1.0$

$<0.15$

3.8
DF Mod

$J 1$
$J 1$
$J V 1$
$J 1$
$J 1$
$J 1$
$J 1$
$J 1$
$J$
$J 1$
$J 1$
$J$

ST $\underline{H}$ Flag Lab

- 0

- 0

- 0

- 0

- 0

- 0

- 0

- 0

0
$-\quad 0$

- 0
$\mathrm{GE}$
$\mathrm{GE}$
$\mathrm{GE}$
$\mathrm{GE}$
$\mathrm{GE}$
$\mathrm{GE}$
$\mathrm{GE}$
$\mathrm{GE}$
$\mathrm{GE}$
$\mathrm{GE}$
$\mathrm{GE}$
$\mathrm{GE}$
$\mathrm{GE}$

Unit

$\mu g h$

$\mu 9$ L

$\mu g / L$

$\mu g / L$

$\mu g h$

$\mu g / L$

$\mu \mathrm{g} / \mathrm{L}$

$\mu g^{2}$

$\mu g / \mathrm{L}$

$\mu g / L$
$\mu g / L$

$\mu \mathrm{g} /$
$\mu \mathrm{g} / \mathrm{L}$

- = exceeded holding time.

- = exceeded standard. 
WELL LFW 43D

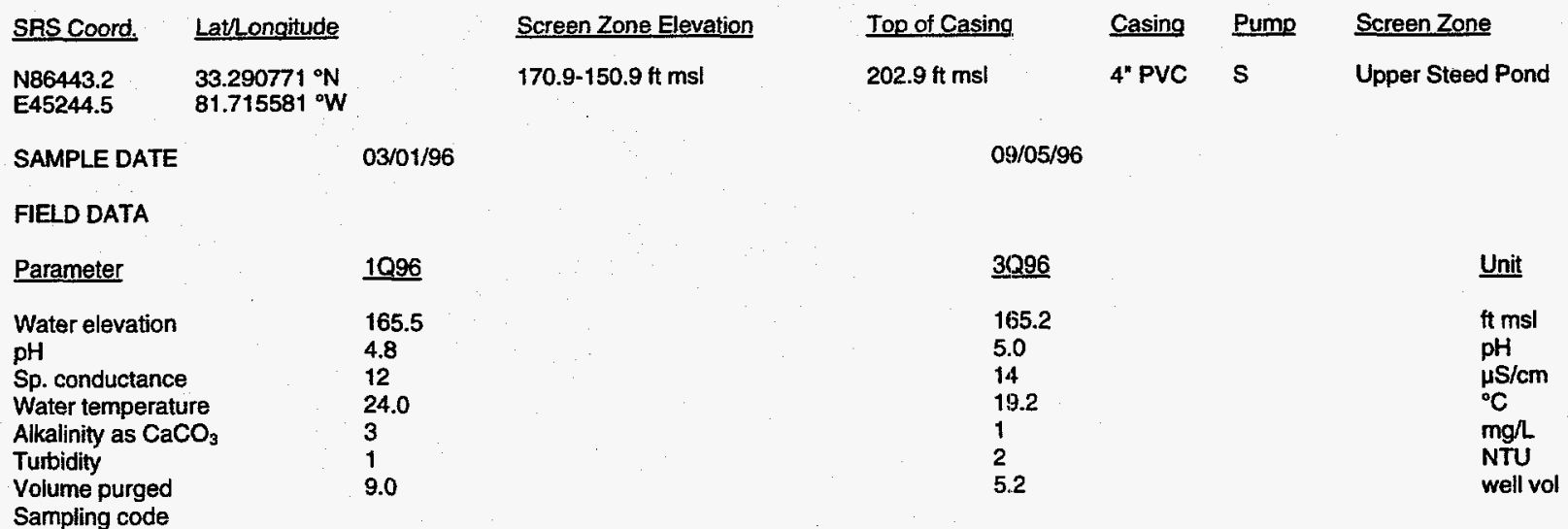

ANALYTICAL DATA

\begin{tabular}{|c|c|c|c|c|c|c|c|c|c|c|c|c|c|c|}
\hline Parameter & 1096 & DF & Mod & $\underline{\text { ST }} \underline{H}$ & Flag & Lab & 3096 & DF & Mod & ST & $\underline{H}$ & Flag & Lab & Unit \\
\hline Acetone & $<10$ & 1 & & & 0 & GE & $<3.9$ & 1 & JV1 & & - & 0 & GE & $\mu g / L$ \\
\hline Acrylonitrile & $<50$ & 1 & & & 0 & GE & $<5.0$ & 1 & J1 & & - & 0 & GE & $\mu g / L$ \\
\hline Antimony, total recoverable & $<0.098$ & 1 & JV & & 0 & GE & $<5.0$ & 1 & & & & 0 & GE & $\mu g /$ \\
\hline Arsenic, total recoverable & $<3.0$ & 1 & J & & 0 & GE & $<5.0$ & 1 & & & & 0 & GE & $\mu g / L$ \\
\hline Barium, total recoverable & 7.3 & 1 & JV & & 0 & GE & 15.8 & 1 & & & & 0 & GE & $\mu g /$ \\
\hline Benzene & $<2.0$ & 1 & & & 0 & GE & $<0.50$ & 1 & J1 & & $\bullet$ & 0 & GE & $\mu g / L$ \\
\hline Beryllium, total recoverable & $<0.30$ & 1 & & & 0 & GE & $<3.0$ & 1 & & & & 0 & GE & $\mu g / L$ \\
\hline Bromochloromethane & & & & & & & $<0.050$ & 1 & J1 & & - & 0 & GE & $\mu g / L$ \\
\hline Bromodichloromethane & $<2.0$ & 1 & & & 0 & GE & $<0.050$ & 1 & Ji & & - & 0 & GE & $\mu g h$ \\
\hline Bromoform & $<2.0$ & 1 & & & 0 & GE & $<0.050$ & 1 & J1 & & $\bullet$ & 0 & GE & $\mu g / L$ \\
\hline Bromomethane & $<2.0$ & 1 & & & 0 & $\mathrm{GE}$ & $<0.10$ & 1 & J1 & & - & 0 & GE & $\mu g / L$ \\
\hline Cadmium, total recoverable & $<0.40$ & 1 & & & 0 & GE & $<2.0$ & 1 & & & & 0 & GE & $\mu g / L$ \\
\hline Carbon disulfide & $<5.0$ & 1 & & & 0 & GE & $<1.0$ & 1 & J1 & & - & 0 & GE & $\mu g h$ \\
\hline Carbon tetrachloride & $<2.0$ & 1 & & & 0 & GE & $<0.050$ & 1 & Jt & & - & 0 & GE & $\mu g h$ \\
\hline Chlorobenzene & $<2.0$ & 1 & & & 0 & GE & $<0.050$ & 1 & J1 & & $\bullet$ & 0 & GE & jgl \\
\hline Chioroethane & $<2.0$ & 1 & & & 0 & GE & $<0.10$ & 1 & J1 & & $\bullet$ & 0 & GE & $\mu g /$ \\
\hline Chloroethene (Vinyl chloride) & $<2.0$ & 1 & & & 0 & GE & $<0.10$ & 1 & J1 & & - & 0 & GE & $\mu g /$ \\
\hline Chloroform & $<2.0$ & 1 & & & 0 & GE & $<0.050$ & 1 & J1 & & $\bullet$ & 0 & GE & $\mu g /$ \\
\hline Chloromethane & $<2.0$ & 1 & & & 0 & GE & $<0.10$ & 1 & J1 & & $\bullet$ & 0 & GE & $\mu g / L$ \\
\hline Chromium, total recoverable & 0.74 & 1 & $\mathbf{J}$ & & 0 & GE & $<4.0$ & 1 & & & & 0 & GE & $\mu g / L$ \\
\hline Cobalt, total recoverable & & & & & & & $<4.0$ & 1 & & & & $\mathbf{0}$ & GE & $\mu g / L$ \\
\hline Copper, total recoverable & 4.9 & 1 & & & 0 & GE & 3.9 & 1 & $\mathrm{~J}$ & & & 0 & GE & $\mu g / L$ \\
\hline Dibromochloromethane & $<2.0$ & 1 & & & 0 & GE & $<0.050$ & 1 & J1 & & - & $\mathbf{0}$ & GE & $\mu g /$ \\
\hline 1,2-Dibromo-3-chioropropane & $<2.0$ & 1 & & & 0 & GE & $<0.050$ & 1 & J1 & & $\bullet$ & 0 & GE & $\mu g / L$ \\
\hline 1,2-Dibromoethane & $<2.0$ & 1 & & & 0 & GE & $<0.050$ & 1 & J1 & & $\bullet$ & 0 & GE & $\mu g / L$ \\
\hline Dibromomethane & $<2.0$ & 1 & & . & 0 & GE & $<0.050$ & 1 & J1 & & $\bullet$ & 0 & GE & $\mu g / L$ \\
\hline 1,2-Dichlorobenzene & & & & & & & $<0.050$ & 1 & J1 & & - & 0 & GE & $\mu g$ \\
\hline 1,4-Dichlorobenzene & & & & & & & $<0.13$ & 1 & JV1 & & $\bullet$ & 0 & GE & $\mu g / 2$ \\
\hline trans-1,4-Dichloro-2-butene & $<2.0$ & 1 & & & 0 & GE & $<0.50$ & 1 & J1 & & $\bullet$ & 0 & GE & $\mu g / 2$ \\
\hline 1,1-Dichioroethane & $<2.0$ & 1 & & & 0 & GE & $<0.050$ & 1 & J1 & & - & 0 & GE & $\mu g / 2$ \\
\hline 1,2-Dichloroethane & $<2.0$ & 1 & & & 0 & GE & $<0.050$ & 1 & J1 & & - & $\mathbf{0}$ & GE & $\mu g / L$ \\
\hline 1,1-Dichloroethylene & $<2.0$ & 1 & & & 0 & GE & $<0.050$ & 1 & J1 & & - & 0 & GE & $\mu g / L$ \\
\hline cis-1,2-Dichloroethylene & & & & & & & $<5.0$ & 1 & J1 & & - & 0 & GE & $\mu g / L$ \\
\hline trans-1,2-Dichloroethylene & $<2.0$ & 1 & & & 0 & GE & $<0.050$ & 1 & J1 & & - & $\mathbf{0}$ & GE & $\mu g /$ \\
\hline Dichloromethane & 1.4 & 1 & J & & 0 & GE & $<1.1$ & 1 & JV1 & & - & 0 & GE & $\mu g / L$ \\
\hline 1,2-Dichloropropane & $<2.0$ & 1 & & & 0 & GE & $<0.050$ & 1 & $\sqrt{1}$ & & - & 0 & - GE & $\mu g / L$ \\
\hline $\begin{array}{l}\text { cis-1,3-Dichioropropene } \\
\text { trans-1,3-Dichioropropene }\end{array}$ & $<2.0$ & 1 & & & 0 & GE & $<0.050$ & 1 & J1 & & - & 0 & GE & \\
\hline Ethylbenzene & $<2.0$ & 1 & & & 0 & GE & $<0.050$ & 1 & J1 & & $\bullet$ & 0 & GE & $\mu g /$ \\
\hline 2-Hexanone & $<10$ & 1 & & & 0 & GE & $<1.0$ & 1 & $\mathbf{J 1}$ & & - & 0 & GE & $\mu g /$. \\
\hline lodomethane (Methyl iodide) & $<10$ & 1 & & & 0 & GE & $<1.0$ & 1 & J1 & & $\bullet$ & 0 & GE & $\mu g h$ \\
\hline Lead, total recoverable & 0.89 & 1 & V & & 0 & GE & 1.3 & 1 & J & & & 0 & GE & $\mu g /$ \\
\hline Methyl ethyl ketone & $<5.0$ & 1 & & & 0 & GE & $<0.25$ & 1 & JV1 & & - & 0 & GE & $\mu g / L$ \\
\hline Methyl isobutyl ketone & $<10$ & 1 & & & 0 & GE & $<1.0$ & 1 & J1 & & $\bullet$ & 0 & GE & $\mu g / L$ \\
\hline Nickel, total recoverable & 0.37 & 1 & JV & & 0 & GE & $<10$ & 1 & & & & 0 & GE & $\mu g / L$ \\
\hline $\mathrm{pH}$ & 5.3 & 1 & J1 & $\bullet$ & 0 & $\mathrm{GE}$ & 5.3 & 1 & J1 & & - & 0 & GE & pH \\
\hline Selenium, total recoverable & $<2.0$ & 1 & & & 0 & GE & $<5.0$ & 1 & & & & 0 & GE & ig/ \\
\hline Silver, total recoverable & $<0.042$ & 1 & V & & 0 & GE & $<2.0$ & 1 & & & & 0 & GE & $\mu g / L$ \\
\hline Specific conductance & 14 & 1 & & & 0 & GE & 17 & 1 & & & & 0 & GE & $\mu S / c n$ \\
\hline Styrene & $<2.0$ & 1 & & & 0 & GE & $<0.050$ & 1 & J1 & & $\bullet$ & 0 & GE & $\mu g / L$ \\
\hline $1,1,1,2-$ Tetrachloroethane & $<2.0$ & 1 & & & 0 & GE & $<0.050$ & 1 & J1 & & $\bullet$ & 0 & GE & $\mu g / L$ \\
\hline
\end{tabular}

$-=$ exceeded holding time.

- = exceeded standard. 
WELL LFW 43D (cont.)

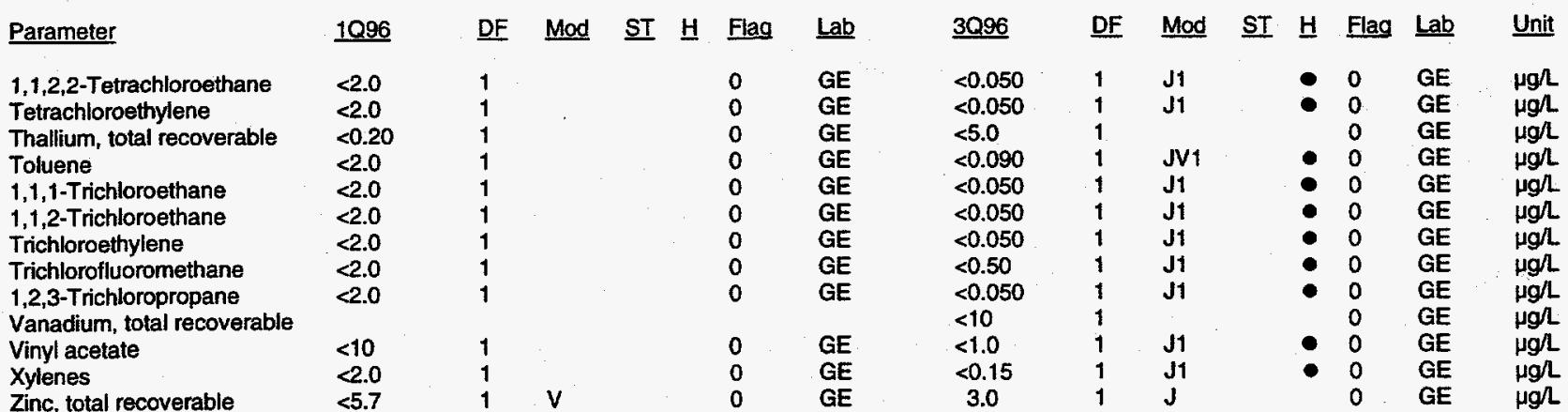

$\checkmark=$ exceeded holding time

= exceeded standard. 
WELL LFW 74C

\begin{tabular}{|c|c|c|c|c|c|c|c|}
\hline SRS Coord. & LatLongitude & & Screen Zone Elevation & Top of Casing & Casing & Pump & Screen Zone \\
\hline $\begin{array}{l}\text { N85813.8 } \\
\text { E45097.8 }\end{array}$ & $\begin{array}{l}33.289140^{\circ} \mathrm{N} \\
81.714743^{\circ} \mathrm{W}\end{array}$ & & $116.0-101.0 \mathrm{ft} \mathrm{msl}$ & $213.6 \mathrm{ft} \mathrm{msl}$ & 4" PVC & $s$ & Middle Steed Pond \\
\hline SAMPLE DATE & & $03 / 05 / 96$ & & $09 / 09 / 96$ & & & \\
\hline \multicolumn{8}{|l|}{ FIELD DATA } \\
\hline Parameter & & 1096 & & 3096 & & & $\underline{\text { Unit }}$ \\
\hline $\begin{array}{l}\text { Water elevation } \\
\text { pH } \\
\text { Sp. conductance } \\
\text { Water temperature } \\
\text { Alkalinity as CaCO } \\
\text { Turbidity } \\
\text { Volume purged } \\
\text { Sampling code }\end{array}$ & & $\begin{array}{l}162.7 \\
5.4 \\
34 \\
20.0 \\
2 \\
1 \\
2.8\end{array}$ & & $\begin{array}{l}200.1 \\
4.8 \\
32 \\
24.0 \\
1 \\
1 \\
3.0\end{array}$ & & & $\begin{array}{l}\text { tt ms! } \\
\mathrm{pH} \\
\mu \mathrm{S} / \mathrm{cm} \\
{ }^{\circ} \mathrm{C} \\
\mathrm{mg} \\
\text { NTU } \\
\text { well vol }\end{array}$ \\
\hline
\end{tabular}

\section{ANALYTICAL DATA}

\begin{tabular}{|c|c|c|c|c|c|c|c|c|c|c|c|c|c|c|c|}
\hline Parameter & 1096 & DF & Mod & $\underline{\mathrm{ST}}$ & 브 & Flag & $\underline{\text { Lab }}$ & $\underline{3096}$ & DF & Mod & $\underline{\text { ST }}$ & H & Flag & $\underline{\text { Lab }}$ & Unit \\
\hline Acetone & $<10$ & 1 & & & & 0 & GE & $<4.0$ & 1 & v & & & 0 & GE & $\mu g / L$ \\
\hline Acrylonitrile & $<50$ & 1 & & & & 0 & $\mathrm{GE}$ & $<5.0$ & 1 & & & & 0 & $\mathrm{GE}$ & $\mu g /$ \\
\hline Antimony, total recoverable & $<5.0$ & 1 & & & & 0 & GE & $<5.0$ & 1 & & & & 0 & GE & $\mu g \Omega$ \\
\hline Arsenic, total recoverable & $<4.1$ & 1 & $v$ & & & 0 & GE & $<5.0$ & 1 & & & & 0 & GE & $\mu g /$ \\
\hline Barium, total recoverable & 5.2 & 1 & & & & 0 & GE & 5.7 & 1 & v & & & 0 & GE & $\mu g /$. \\
\hline Benzene & $<2.0$ & 1 & $\mathrm{~J}$ & & & 0 & GE & $<0.50$ & 1 & & & & 0 & GE & $\mu g /$ \\
\hline Beryllium, total recoverable & 0.060 & 1 & J & & & 0 & GE & $\begin{array}{l}0.26 \\
<0.050\end{array}$ & 1 & J & & & 0 & WA & $\mu g h$ \\
\hline & $<2.0$ & 1 & & & & 0 & & $\begin{array}{l}<0.050 \\
<0.050\end{array}$ & 1 & & & & 0 & GE & $\mu g / 2$ \\
\hline $\begin{array}{l}\text { Bromodichloromethane } \\
\text { Bromoform }\end{array}$ & $<2.0$ & $\begin{array}{l}1 \\
1\end{array}$ & & & & $\begin{array}{l}0 \\
0\end{array}$ & $\begin{array}{l}\mathrm{GE} \\
\mathrm{GE}\end{array}$ & $<0.050$ & $\begin{array}{l}1 \\
1\end{array}$ & & & & $\begin{array}{l}0 \\
0\end{array}$ & $\begin{array}{l}\mathrm{GE} \\
\mathrm{GE}\end{array}$ & $\begin{array}{l}\mu g h L \\
\mu g / L\end{array}$ \\
\hline Bromomethane & $<2.0$ & 1 & & & & 0 & $\mathrm{GE}$ & $=0.10$ & $i$ & & & & 0 & GE & $\mu g /$ \\
\hline Cadmium, total recoverable & $<0.12$ & 1 & v & & & 0 & GE & $<2.0$ & 1 & & & & 0 & $\overline{G E}$ & $\mu g / L$ \\
\hline Carbon disulfide & $<5.0$ & 1 & & & & 0 & $\mathrm{GE}$ & $\leqslant 1.0$ & 1 & & & & 0 & GE & $\mu \mathrm{g} / \mathrm{L}$ \\
\hline Carbon tetrachloride & $<2.0$ & 1 & & & & 0 & GE & $=0.050$ & 1 & & & & 0 & GE & $\mu g / L$ \\
\hline Chlorobenzene & $<2.0$ & 1 & & & & 0 & $\mathrm{GE}$ & $<0.050$ & $i$ & & & & 0 & GE & $\mu \mathrm{g} / \mathrm{L}$ \\
\hline Chloroethane & $<2.0$ & 1 & & & & 0 & GE & $<0.10$ & 1 & & & & 0 & GE & $\mu g / L$ \\
\hline Chloroethene (Vinyl chloride) & $<2.0$ & 1 & & & & 0 & $\overline{G E}$ & $<0.10$ & 1 & & & & 0 & GE & $\mu g h$ \\
\hline Chloroform & $<2.0$ & 1 & & & & 0 & GE & $<0.050$ & 1 & & & & 0 & GE & $\mu g /$ \\
\hline Chloromethane & $<2.0$ & 1 & & & & 0 & GE & 1.1 & 1 & $\mathrm{~J}$ & & & 0 & GE & $\mu g h$ \\
\hline Chromium, total recoverable & $<4.0$ & 1 & & & & 0 & GE & $<1.0$ & 1 & v & & & 0 & WA & $\mu g / L$ \\
\hline Cobalt, total recoverable & $<0.82$ & 1 & $\mathrm{v}$ & & & 0 & $\mathrm{GE}$ & $<0.30$ & 1 & $v$ & & & 0 & $\mathrm{GE}$ & $\mu g / L$ \\
\hline Copper, total recoverable & 5.2 & 1 & & & & 0 & GE & 17 & 1 & & & & 0 & WA & $\mu g h \mathrm{~L}$ \\
\hline Dibromochloromethane & $<2.0$ & 1 & & & & 0 & GE & $<0.050$ & 1 & & & & 0 & GE & $\mu g h$ \\
\hline 1,2-Dibromo-3-chloropropane & $<2.0$ & 1 & & & & 0 & $\mathrm{GE}$ & $<0.050$ & $i$ & & & & 0 & $\mathrm{GE}$ & $\mu g h$ \\
\hline 1,2-Dibromoethane & $<2.0$ & $i$ & & & & 0 & $\mathrm{GE}$ & $<0.050$ & 1 & & & & 0 & $G E$ & $\mu g h$ \\
\hline Dibromomethane & $<2.0$ & 1 & & & & 0 & GE & $<0.050$ & 1 & & & & 0 & GE & $\mu g / L$ \\
\hline 1,2-Dichlorobenzene & & & & & & & & $<0.050$ & 1 & & & & 0 & GE & $\mu g h$ \\
\hline 1,4-Dichlorobenzene & & & & & & & & $<0.050$ & 1 & & & & 0 & $\mathrm{GE}$ & $\mu g h$ \\
\hline trans-1,4-Dichloro-2-butene & $<2.0$ & 1 & & & & 0 & $\mathrm{GE}$ & $<0.50$ & 1 & & & & 0 & GE & $\mu g / L$ \\
\hline 1,1-Dichloroethane & $<2.0$ & 1 & & & & 0 & $\mathrm{GE}$ & $<0.050$ & 1 & & & & 0 & $\mathrm{GE}$ & $\mu g / L$ \\
\hline 1,2-Dichloroethane & $<2.0$ & 1 & & & & 0 & GE & $<0.050$ & 1 & & & & 0 & GE & $\mu g / L$ \\
\hline 1,1 -Dichloroethylene & $<2.0$ & 1 & & & & 0 & GE & $<0.050$ & 1 & & & & 0 & $\mathrm{GE}$ & $\mu g / L$ \\
\hline cis-1,2-Dichloroethylene & & & & & & & & $<2.0$ & 1 & & & & 0 & GE & $\mu g / L$ \\
\hline trans-1,2-Dichloroethylene & $<2.0$ & 1 & & & & 0 & GE & $<0.050$ & 1 & & & & 0 & $\mathrm{GE}$ & $\mu g / L$ \\
\hline Dichloromethane & $<10$ & 1 & & & & 0 & $\mathrm{GE}$ & $<1.2$ & 1 & v & & & 0 & WA & $\mu g / L$ \\
\hline 1,2-Dichloropropane & $<2.0$ & 1 & & & & 0 & GE & $<0.050$ & 1 & & & & 0 & GE & $\mu g / L$ \\
\hline $\begin{array}{l}\text { cis-1,3-Dichloropropene } \\
\text { trans-1,3-Dichloropropene }\end{array}$ & $<2.0$ & 1 & & & & 0 & GE & $<0.050$ & $i$ & & & & 0 & GE & $\mu g / L$ \\
\hline $\begin{array}{l}\text { Ethylbenzene } \\
\text { Ello }\end{array}$ & $<2.0$ & 1 & & & & 0 & GE & $<0.050$ & 1 & & & & 0 & GE & $\mu g / L$ \\
\hline 2-Hexanone & $<10$ & 1 & & & & 0 & GE & $<1.0$ & 1 & & & & 0 & $\mathrm{GE}$ & $\mu g / 2$ \\
\hline lodomethane (Methyl iodide) & $<10$ & 1 & & & & 0 & GE & $<0.22$ & 1 & v & & & 0 & $\mathrm{GE}$ & $\mu g /$ \\
\hline Lead, total recoverable & $<2.9$ & $i$ & $v$ & & & 0 & $\mathrm{GE}$ & 3.4 & 1 & J & & & 0 & GE & $\mu g / \mathrm{h}$ \\
\hline Methyl ethyl ketone & $<5.0$ & 1 & & & & 0 & GE & $<1.0$ & 1 & & & & 0 & GE & $\mu g \mathrm{~L}$ \\
\hline Methyl isobutyl ketone & $<10$ & 1 & & & & 0 & $\mathrm{GE}$ & $<1.0$ & 1 & & & & 0 & $\mathrm{GE}$ & $\mu \mathrm{g} / \mathrm{L}$ \\
\hline Nickel, total recoverable & $<10$ & 1 & & & & 0 & GE & $<10$ & 1 & & & & 0 & GE & \\
\hline $\mathrm{pH}$ & 5.2 & 1 & $\mathrm{~J} 1$ & & - & 0 & GE & 5.4 & 1 & $\mathrm{~J}$ & & - & 0 & WA & $\mathrm{pH}$ \\
\hline Selenium, total recoverable & $<5.0$ & 1 & & & & 0 & GE & $<5.0$ & 1 & & & & 0 & GE & $\mu \mathrm{g} / \mathrm{L}$ \\
\hline Silver, total recoverable & $<2.0$ & 1 & & & & 0 & GE & $<0.67$ & 1 & $v$ & & & 0 & WA & $\mu g / L$ \\
\hline Specific conductance & 35 & 1 & & & & 0 & GE & 35 & 1 & & & & 0 & GE & \\
\hline Styrene & $<2.0$ & 1 & & & & 0 & $\mathrm{GE}$ & $<0.050$ & 1 & & & & 0 & GE & $\mu g / L$ \\
\hline 1,1,1,2-Tetrachloroethane & $<2.0$ & 1 & & & & 0 & GE & $<0.050$ & 1 & & & & 0 & GE & $\mu g / L$ \\
\hline
\end{tabular}

$\overline{0}=$ exceeded holding time.

= exceeded standard. 


\section{WELL LFW 74C (cont.)}

Parameter

1,1,2,2-Tetrachloroethane Tetrachloroethylene

Thallium, total recoverabie

Toluene

1,1,1-Trichloroethane

1,1,2-Trichloroethane

Trichloroethylene

Trichlorofluoromethane

1,2,3-Trichloropropane

Vanadium, total recoverabie

Vinyl acetate

Xylenes

Zinc, total recoverable
1096

$<2.0$

$<2.0$

$<5.0$

$<2.0$

$<2.0$

20

$<20$

$<2.0$

$<2.0$

$<10$

$<10$

$<2.0$
DF Mod ST 브 Flag Lab

GE $\quad<0.050$

$<0.050$

$<5.0$

$<0.27$

$<0.050$

$<0.050$

$<0.050$

$<0.50$

$<0.050$

$<6.9$

$<1.0$

$<0.15$

15
DF

Mod

H Flag Lab

GE $\mu g /$

GE $\mu g /$

V

GE

GE

GE

GE

GE

WA

GE

WA
Unit

$\mu g / L$

$\mu g / L$

$\mu g / L$

ug/L

$\mu g /$

$\mu \mathrm{g} / \mathrm{L}$

$\mu g /$

$\mu g /$

$\mu g / L$

$\mu g / L$

- = exceeded holding time.

u = exceeded standard. 
WELL LFW 74D

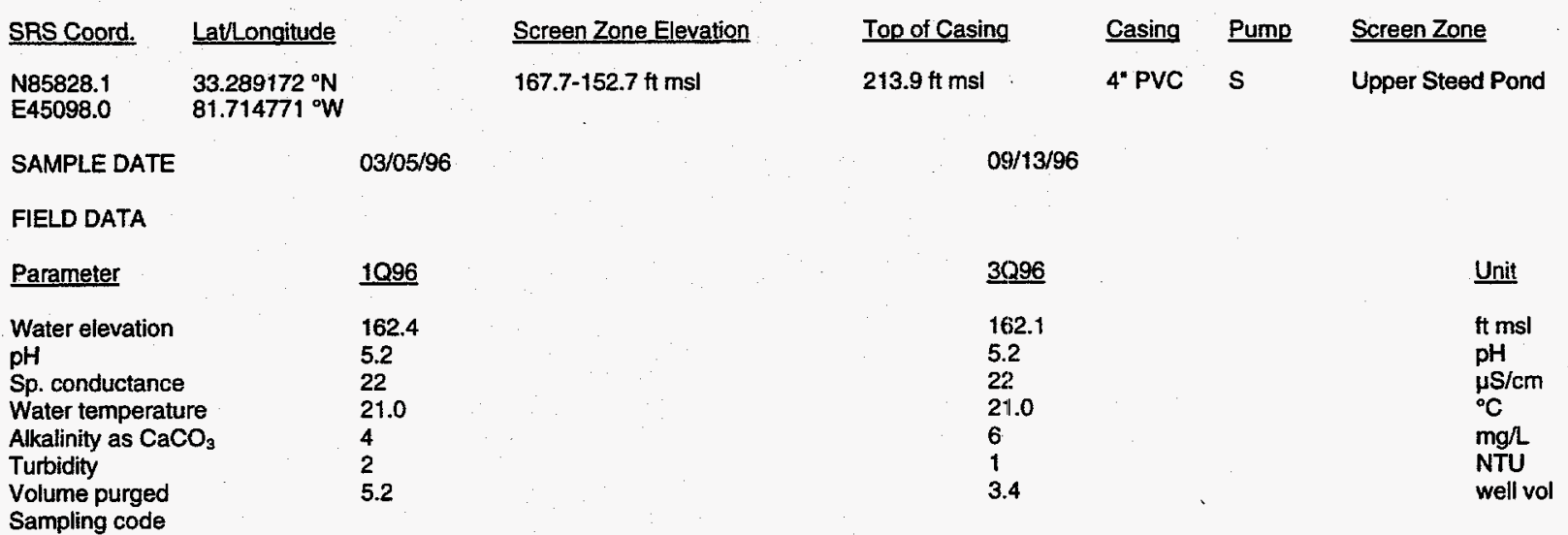

ANALYTICAL DATA

\begin{tabular}{|c|c|c|c|c|c|c|c|c|c|c|c|c|c|c|}
\hline Parameter & $\underline{1096}$ & DF & Mod & ST & $\underline{\mathbf{H}}$ & Flag & Lab & $\underline{3096}$ & $\underline{\mathrm{DF}}$ & Mod & ST $\underline{H}$ & Flag & Lab & Unit \\
\hline Acetone & $<3 . t$ & 1 & v & & & 0 & WA & 26 & 1 & $v$ & & 0 & GE & $\mu g h$ \\
\hline Acrylonitrile & $<10$ & 1 & & & & 0 & WA & $<5.0$ & 1 & & & 0 & GE & $\mu g / L$ \\
\hline Antimony, total recoverable & $<2.3$ & 1 & v & & & 0 & GE & $<5.0$ & 1 & & & 0 & GE & $\mu g h$ \\
\hline Arsenic, total recoverable & $<4.5$ & 1 & $v$ & & & 0 & GE & $<5.0$ & 1 & & & 0 & GE & $\mu g / L$ \\
\hline Barium, total recoverable & 6.1 & 1 & & & & 0 & WA & 6.2 & 1 & $v$ & & 0 & GE & $\mu g / L$ \\
\hline Benzene & $<2.0$ & 1 & & & & 0 & GE & $<0.50$ & 1 & & & 0 & GE & $\mu \mathrm{g} / \mathrm{L}$ \\
\hline Beryllium, total recoverable & $<1.6$ & 1 & & & & 0 & WA & $<3.0$ & 1 & & & 0 & GE & $\mu g /$ \\
\hline Bromochloromethane & $<5.0$ & 1 & & & & 0 & WA & $<0.050$ & 1 & & & 0 & GE & $\mu g / L$ \\
\hline Bromodichloromethane & $<2.0$ & 1 & & & & 0 & GE & $<0.050$ & 1 & & & 0 & GE & $\mu g / L$ \\
\hline Bromoform & $<2.0$ & 1 & & & & 0 & GE & $<0.050$ & 1 & & & 0 & GE & $\mu g \Omega$ \\
\hline Bromomethane & $<2.0$ & 1 & & & & 0 & GE & $<0.10$ & 1 & & & 0 & GE & $\mu g h$ \\
\hline Cadmium, total recoverable & $<0.13$ & 1 & $v$ & & & 0 & GE & $<2.0$ & 1 & & & 0 & GE & $\mu g /$ \\
\hline Carbon disulfide & $<5.0$ & 1 & & & & 0 & GE & 0.19 & 1 & $\mathrm{~J}$ & & 0 & GE & $\mu g /$ \\
\hline Carbon tetrachloride & $<2.0$ & 1 & & & & $\mathbf{0}$ & GE & $<0.050$ & 1 & & & 0 & GE & $\mu g /$ \\
\hline Chlorobenzene & $<2.0$ & 1 & & & & 0 & GE & $<0.050$ & 1 & & & 0 & GE & $\mu g h$ \\
\hline Chloroethane & $<2.0$ & 1 & & & & 0 & GE & $<0.10$ & 1 & & & 0 & GE & $\mu g \Omega$ \\
\hline Chloroethene (Vinyl chloride) & $<2.0$ & 1 & & & & 0 & GE & $<0.10$ & 1 & & & 0 & GE & $\mu g / L$ \\
\hline Chloroform & $<2.0$ & 1 & & & & 0 & GE & 0.080 & 1 & & & 0 & GE & $\mu g /$ \\
\hline Chloromethane & $<2.0$ & 1 & & & & 0 & GE & $<0.10$ & 1 & & & 0 & GE & $\mu g / L$ \\
\hline Chromium, total recoverable & 2.0 & 1 & $\mathrm{~J}$ & & & 0 & WA & 0.94 & 1 & $J$ & & 0 & GE & $\mu g / L$ \\
\hline Cobalt, total recoverable & $<0.54$ & 1 & v & & & 0 & GE & $<0.40$ & 1 & v & & 0 & GE & $\mu g / L$ \\
\hline Copper, total recoverable & 4.5 & 1 & & & & 0 & GE & 1.4 & 1 & $\mathbf{J}$ & & 0 & GE & $\mu g / L$ \\
\hline Dibromochloromethane & $<2.0$ & 1 & & & & 0 & GE & $<0.050$ & 1 & & & 0 & GE & $\mu g / L$ \\
\hline 1,2-Dibromo-3-chloropropane & $<2.0$ & 1 & & & & 0 & GE & $<0.050$ & 1 & & & 0 & GE & $\mu g / L$ \\
\hline 1,2-Dibromoethane & $<2.0$ & 1 & & & & 0 & GE & $<0.050$ & 1 & & & 0 & GE & $\mu g / L$ \\
\hline Dibromomethane & $<2.0$ & 1 & & & & 0 & GE & $<0.050$ & 1 & & & 0 & GE & $\mu g / L$ \\
\hline 1,2-Dichlorobenzene & $<5.0$ & 1 & & & & 0 & WA & $<0.050$ & 1 & & & 0 & GE & $\mu g / L$ \\
\hline 1,4-Dichlorobenzene & $<5.0$ & 1 & & & & 0 & WA & 0.070 & 1 & & & 0 & GE & $\mu g / L$ \\
\hline trans-1,4-Dichloro-2-butene & $<2.0$ & 1 & & & & 0 & GE & $<0.50$ & 1 & & & 0 & GE & $\mu g / L$ \\
\hline 1,1-Dichloroethane & $<2.0$ & 1 & & & & 0 & GE & 0.13 & 1 & & & 0 & GE & $\mu g / L$ \\
\hline 1,2-Dichloroethane & $<2.0$ & 1 & & & & 0 & GE & $<0.050$ & 1 & & & 0 & GE & $\mu g / L$ \\
\hline 1,1-Dichloroethylene & $<2.0$ & 1 & & & & 0 & GE & 0.43 & 1 & & & 0 & GE & $\mu g / L$ \\
\hline cis-1,2-Dichloroethylene & $<5.0$ & 1 & & & & 0 & WA & $<5.0$ & 1 & & & 0 & GE & $\mu g / L$ \\
\hline trans-1,2-Dichloroethylene & $<2.0$ & 1 & & & & 0 & GE & $<0.050$ & 1 & & & 0 & GE & $\mu g / L$ \\
\hline Dichloromethane & $<5.0$ & 1 & & & & 0 & WA & $<3.0$ & 1 & v & & 0 & GE & $\mu g / L$ \\
\hline 1,2-Dichloropropane & $<2.0$ & 1 & & & & 0 & GE & $<0.050$ & 1 & & & 0 & GE & $\mu g / L$ \\
\hline cis-1,3-Dichloropropene & $<2.0$ & 1 & & & & 0 & $G E$ & $<0.050$ & 1 & & & 0 & GE & $\mu g / L$ \\
\hline trans-1,3-Dichloropropene & $<2.0$ & 1 & & & & 0 & GE & & & & & & & $\mu g / L$ \\
\hline Ethylbenzene & $<2.0$ & 1 & & & & 0 & GE & $<13.050$ & 1 & & & $\mathbf{0}$ & GE & $\mu g / L$ \\
\hline 2-Hexanone & $<10$ & 1 & & & & 0 & GE & $<1.0$ & 1 & & & 0 & GE & $\mu g /$ \\
\hline lodomethane (Methyl iodide) & $<5.0$ & 1 & & & & 0 & WA & $<0.24$ & 1 & v & & 0 & GE & $\mu g / L$ \\
\hline Lead, total recoverable & 8.6 & 1 & v & & & 0 & GE & 5.3 & 1 & & & 0 & GE & $\mu g / L$ \\
\hline Methyl ethyl ketone & $<5.0$ & 1 & & & & 0 & GE & $<1.0$ & 1 & & & 0 & GE & $\mu g h$ \\
\hline Methyl isobutyl ketone & $<10$ & 1 & & & & 0 & GE & 0.32 & 1 & $\mathbf{J}$ & & & GE & $\mu g / L$ \\
\hline Nickel, total recoverable & 4.1 & 1 & $\mathrm{~J}$ & & & 0 & WA & $<10$ & 1 & & & 0 & GE & $\mu g / 2$ \\
\hline $\mathrm{pH}$ & 5.7 & 1 & $J$ & & - & 0 & WA & 5.0 & 1 & J1 & - & 0 & GE & $\mathrm{pH}$ \\
\hline Selenium, total recoverable & $<5.0$ & 1 & & & & 0 & GE & 1.8 & 1 & $\mathbf{J}$ & & 0 & GE & $\mu g /$ \\
\hline Silver, total recoverable & 1.1 & 1 & $\mathbf{J}$ & & & 0 & WA & $<2.0$ & 1 & & & 0 & GE & $\mu g / L$ \\
\hline Specific conductance & 33 & 1 & & & & 0 & GE & 24 & 1 & & & 0 & GE & $\mu \mathrm{S} / \mathrm{cm}$ \\
\hline Styrene & $<2.0$ & 1 & & & & 0 & GE & $<0.050$ & 1 & & & 0 & GE & $\mu g /$ L \\
\hline 1,1,1,2-Tetrachloroethane & $<2.0$ & 1 & & & & 0 & GE & $<0.050$ & 1 & & & 0 & GE & $\mu g / L$ \\
\hline
\end{tabular}

= exceeded holding time.

- = exceeded standard. 
WELL LFW 74D (cont.)

Parameter

1,1,2,2-Tetrachloroethane Tetrachloroethylene

Thallium, total recoverable

Toluene

1,1,1-Trichloroethane

1,1,2-Trichloroethane

Trichloroethylene

Trichlorolluoromethane

1,2,3-Trichloropropane

Vanadium, total recoverable

Vinyl acetate

Xylenes

Zinc, total recoverable
1096

$<2.0$

$<2.0$

$<5.0$

$<20$

2.5

$<2.0$

$<2.0$

15

$<2.0$

$<6.9$

$<10$

$<2.0$

54
DF Mad SI H Flag Lab

GE $\quad<0.050$

GE $\quad 0.21$

GE

$<0.24$

WA 6.4

GE $\quad<0.050$

0.090

39

$<0.050$

$<10$

$<1.0$

0.080

0.080
23
DF Mod ST $\underline{\text { H Flag Lab }}$

GE
GE
GE
GE
GE
GE
GE
$G E$
$G E$
$G E$
$G E$
$G E$

$\mu g /$

$\mu g / L$

$\mu g / L$

$\mu g / L$

$\mu g /$.

$\mu g /$

$\mu g h$

$\mu g /$.

$\mu g / L$

$\mu g h$

$\mu g / L$

$\mu g /$.

$\mu g / L$

$-=$ exceeded holding time.

- = exceeded standard. 
WELL LFW 75C

$\begin{array}{ll}\text { SRS Coord. } & \text { Lat/Longitude } \\ \text { N85856.8 } & \\ \text { E45357.0 } & 83.289658^{\circ} \mathrm{N} \\ & 81.714145^{\circ} \mathrm{W}\end{array}$

SAMPLE DATE

FIELD DATA
Screen Zone Elevation

$115.6-100.6 \mathrm{ft} \mathrm{msl}$

03/08/96

1096

162.3

5.2

34

19.0

1

2.5

$\begin{array}{llll}\text { Top of Casing } & \text { Casing } & \text { Pump } & \text { Screen Zone } \\ 197.8 \mathrm{ft} \mathrm{msl} & 4^{n} \text { PVC } & S & \text { Middle Steed Pond }\end{array}$

$09 / 09 / 96$

3Q996

Unit

161.8

4.8

32

1

1

ft ms!

$\mathrm{pH}$

$\mu$ S/cm

NTS

well vol

Volume purged

ANALYTICAL DATA

\begin{tabular}{|c|c|c|c|c|c|c|c|c|c|c|c|c|c|c|c|}
\hline Parameter & 1096 & DF & Mod & $\underline{\mathrm{ST}}$ & $\underline{\mathrm{H}}$ & Flag & $\underline{\text { Lab }}$ & 3096 & $\underline{D F}$ & Mod & $\underline{\text { ST }}$ & 브 & Flag & Lab & Unit \\
\hline Acetone & $<10$ & 1 & & & & 0 & GE & $<4.4$ & 1 & v & & & 0 & GE & $\mu g / L$ \\
\hline Acrylonitrile & $<50$ & 1 & & & & 0 & GE & $<5.0$ & 1 & & & & 0 & GE & $\mu g / L$ \\
\hline Antimony, total recoverable & $<5.0$ & 1 & & & & 0 & GE & 5.2 & 1 & & & & 1 & GE & $\mu g /$ \\
\hline Arsenic, total recoverable & $<5.0$ & 1 & & & & 0 & GE & $<5.0$ & 1 & & & & 0 & GE & $\mu g h$ \\
\hline Barium, total recoverable & 7.0 & 1 & V & & & 0 & GE & 8.3 & 1 & V & & & 0 & GE & $\mu g / L$ \\
\hline Benzene & $<2.0$ & 1 & & & & 0 & GE & $<0.50$ & 1 & & & & 0 & $\mathrm{GE}$ & $\mu g / L$ \\
\hline $\begin{array}{l}\text { Beryllium, total recoverable } \\
\text { Bromochloromethane }\end{array}$ & $<0.18$ & 1 & JV & & & 0 & GE & $\begin{array}{c}0.77 \\
<0.050\end{array}$ & $\begin{array}{l}1 \\
1\end{array}$ & $\mathbf{J}$ & & & $\begin{array}{l}0 \\
0\end{array}$ & GE & $\mu g / 2$ \\
\hline Bromodichloromethane & $<2.0$ & 1 & & & & 0 & GE & $<0.050$ & 1 & & & & 0 & $\begin{array}{l}\text { GE } \\
\text { GE }\end{array}$ & $\begin{array}{l}\mu g / \\
\mu g / L\end{array}$ \\
\hline Bromotorm & $<2.0$ & 1 & & & & 0 & GE & $<0.050$ & 1. & & & & 0 & GE & $\mu g /$ \\
\hline Bromomethane & $<2.0$ & 1 & & & & 0 & GE & $<0.10$ & 1 & & & & 0 & GE & $\mu g / L$ \\
\hline Cadmium, total recoverable & $<2.0$ & 1 & J & & & 0 & GE & 0.59 & 1 & $J$ & & & 0 & GE & $\mu g / L$ \\
\hline Carbon disulfide & $<5.0$ & 1 & & & & 0 & GE & $<1.0$ & 1 & & & & 0 & GE & $\mu g / L$ \\
\hline Carbon tetrachloride & $<2.0$ & 1 & & & & 0 & GE & $<0.050$ & 1 & & & & 0 & GE & $\mu g / L$ \\
\hline Chlorobenzene & $<2.0$ & 1 & & & & 0 & GE & $<0.050$ & 1 & & & & 0 & GE & $\mu g / L$ \\
\hline Chloroethane & $<2.0$ & 1 & & & & 0 & GE & $<0.10$ & 1 & & & & 0 & GE & $\mu g /$ \\
\hline Chloroethene (Vinyl chloride) & $<2.0$ & 1 & & & & 0 & GE & $<0.10$ & 1 & & & & 0 & GE & $\mu g / L$ \\
\hline Chloroform & $<2.0$ & 1 & & & & 0 & GE & $<0.050$ & 1 & & & & 0 & $\mathbf{G E}$ & $\mu g / L$ \\
\hline Chloromethane & $<2.0$ & 1 & & & & 0 & GE & $<0.10$ & 1 & & & & 0 & GE & $\mu g /$ \\
\hline Chromium, total recoverable & $<4.0$ & 1 & & & & 0 & GE & 0.99 & 1 & $J$ & & & 0 & GE & $\mu g / L$ \\
\hline Cobalt, total recoverable & $<1.1$ & 1 & V & & & 0 & GE & $<2.2$ & 1 & JV & & & 0 & GE & $\mu g / L$ \\
\hline Copper, total recoverable & $<0.71$ & 1 & V & & & 0 & $\mathrm{GE}$ & 1.6 & 1 & J & & & 0 & GE & $\mu g /$ \\
\hline Dibromochloromethane & $<2.0$ & 1 & & & & 0 & GE & $<0.050$ & 1 & & & & 0 & GE & $\mu g \Omega$ \\
\hline 1,2-Dibromo-3-chloropropane & $<2.0$ & 1 & & & & 0 & GE & $<0.050$ & 1 & & & & 0 & GE & $\mu g /$ \\
\hline 1,2-Dibromoethane & $<2.0$ & 1 & & & & 0 & GE & $<0.050$ & 1 & & & & 0 & GE & $\mu g / L$ \\
\hline Dibromomethane & $<2.0$ & 1 & & & & 0 & GE & $<0.050$ & 1 & & & & 0 & GE & $\mu g /$ \\
\hline 1,2-Dichlorobenzene & & & & & & & & $<0.050$ & 1 & & & & 0 & GE & $\mu g / L$ \\
\hline 1,4-Dichlorobenzene & & & & & & & & $<0.050$ & 1 & & & & 0 & GE & $\mu g / L$ \\
\hline trans-1,4-Dichloro-2-butene & $<2.0$ & 1 & & & & 0 & GE & $<0.50$ & 1 & & & & 0 & GE & $\mu g / L$ \\
\hline 1,1-Dichloroethane & $<2.0$ & 1 & & & & 0 & GE & $<0.050$ & 1 & & & & 0 & GE & $\mu g / L$ \\
\hline 1,2-Dichtoroethane & $<2.0$ & 1 & & & & 0 & GE & $<0.050$ & 1 & & & & 0 & GE & $\mu g / L$ \\
\hline 1,1-Dichloroethylene & $<2.0$ & 1 & & & & 0 & GE & $<0.050$ & 1 & & & & 0 & GE & $\mu g /$ \\
\hline cis-1,2-Dichloroethylene & & & & & & & & $<5.0$ & 1 & & & & 0 & GE & $\mu g /$ \\
\hline trans-1,2-Dichloroethylene & $<2.0$ & 1 & & & & 0 & GE & $<0.050$ & 1 & & & & 0 & GE & $\mu g / L$ \\
\hline Dichloromethane & $<10$ & 1 & & & & 0 & $\mathrm{GE}$ & $<3.3$ & 1 & V & & & 0 & GE & $\mu g /$ \\
\hline 1,2-Dichloropropane & $<2.0$ & 1 & & & & 0 & GE & $<0.050$ & 1 & & & & 0 & GE & $\mu g / L$ \\
\hline $\begin{array}{l}\text { cis-1,3-Dichloropropene } \\
\text { trans-1,3-Dichioropropene }\end{array}$ & $<2.0$ & 1 & & & & 0 & GE & $<0.050$ & 1 & & & & 0 & GE & $\mu g /$ \\
\hline Ethylbenzene & $<2.0$ & 1 & & & & 0 & GE & $<0.050$ & 1 & & & & 0 & GE & $\mu g / L$ \\
\hline 2-Hexanone & $<10$ & 1 & & & & 0 & GE & $<1.0$ & 1 & & & & 0 & GE & $\mu g /$ \\
\hline lodomethane (Methyl iodide) & $<10$ & 1 & & & & 0 & GE & $<0.20$ & 1 & V & & & 0 & GE & $\mu g / L$ \\
\hline Lead, total recoverable & $<5.0$ & 1 & & & & 0 & GE & 3.8 & 1 & $\mathbf{J}$ & & & 0 & GE & $\mu g /$ \\
\hline Methyl ethyl ketone & $<5.0$ & 1 & & & & 0 & GE & $<1.0$ & 1 & & & & 0 & GE & $\mu g /$ \\
\hline Methyl isobutyl ketone & $<10$ & 1 & & & & 0 & GE & $<1.0$ & 1 & & & & 0 & GE & $\mu g /$ \\
\hline Nickel, total recoverable & 1.2 & 1 & $J$ & & & 0 & GE & 0.97 & 1 & $J$ & & & 0 & GE & $\mu g /$ \\
\hline pH & 5.3 & 1 & J1 & & - & 0 & $G E$ & 5.2 & 1 & J1 & & - & 0 & GE & $\mathrm{pH}$ \\
\hline Selenium, total recoverable & $<5.0$ & 1 & & & & 0 & GE & $<5.0$ & 1 & & & & 0 & GE & $\mu g / L$ \\
\hline Silver, total recoverable & $<2.0$ & 1 & & & & 0 & GE & $<2.0$ & 1 & & & & 0 & GE & Hgll \\
\hline Specific conductance & 31 & 1 & & & & 0 & GE & 33 & 1 & & & & 0 & GE & $\mu \mathrm{S} / \mathrm{cm}$ \\
\hline Styrene & $<2.0$ & 1 & & & & 0 & GE & $<0.050$ & 1 & & & & 0 & GE & $\mu g / L$ \\
\hline 1,1,1,2-Tetrachloroethane & $<2.0$ & 1 & & & & 0 & GE & $<0.050$ & 1 & & & & 0 & GE & $\mu g /$ \\
\hline
\end{tabular}

- = exceeded holding time.

n = exceeded standard. 
WELL LFW 75C (cont.)

Parameter

1,1,2,2-Tetrachloroethane Tetrachloroethylene

Thallium, total recoverable

Toluene

1,1,1-Trichloroethane

1,1,2-Trichloroethane

Trichloroethylene

Trichlorofluoromethane

1,2,3-Trichloropropane

Vanadium, total recoverable

Vinyl acetate

Xylenes

Zinc, total recoverable
109

$<2.0$

$<2.0$

20

2.0

$<2.0$

$<2.0$

$<2.0$

$<2.0$

$<2.0$

$<0.72$

$<10$

$<2.0$

32
J

V

V
ST H Flag Lab

GE $\quad<0.050$

0.060

$<5.7$

$<0.29$

$<0.050$

$<0.050$

$<0.050$

$<0.50$

$<0.050$

$<0.82$

$<1.0$

$<0.15$

26
DF Mod ST $\underline{H}$ Flag Lab Unit

V

V

V

GE
$G E$
GE
$\mathrm{GE}$
$\mathrm{GE}$
$\mathrm{GE}$
$\mathrm{GE}$
$\mathrm{GE}$
$\mathrm{GE}$
$\mathrm{GE}$
$\mathrm{GE}$
$\mathrm{GE}$
$\mathrm{GE}$

ugh

$\mu g / \mathrm{L}$

$\mu g h$

Hgl

$\mu g h$

$\mu g / L$

$\mu g /$ L

$\mu g / L$

$\mu g / 2$

$\mu g /$.

$\mu g / L$

$\mu g / 2$

$\mu g / 2$

$0=$ exceeded holding time.

u = exceeded standard. 
WELL LFW 75D

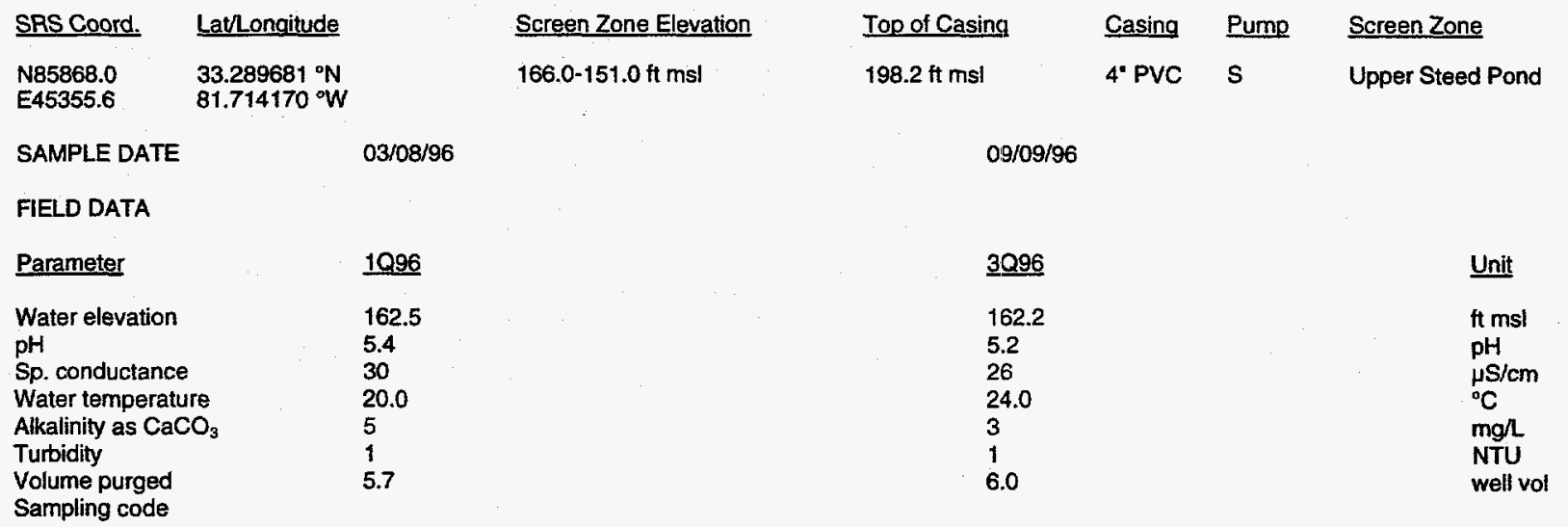

ANALYTICAL DATA

\begin{tabular}{|c|c|c|c|c|c|c|c|c|c|c|c|c|c|c|}
\hline Parameter & 1096 & $\underline{\mathrm{DF}}$. & Mod & $\underline{\mathrm{ST}}$ & $\underline{\mathrm{H}}$ & Flag & $\underline{L a b}$ & 3096 & DF & Mod & $\underline{\text { ST }} \underline{H}$ & Flag & $\underline{\text { Lab }}$ & Unit \\
\hline Acetone & $<10$ & 1 & & & & 0 & GE & $<6.2$ & 1 & $\mathrm{v}$ & & 0 & GE & $\mu g /$ \\
\hline Acrylonitrile & $<50$ & 1 & & & & 0 & GE & $<5.0$ & 1 & & & 0 & GE & $\mu g /$ \\
\hline Antimony, total recoverable & $<5.0$ & 1 & & & & 0 & GE & 6.1 & 1 & & & 2 & GE & $\mu g \Omega$ \\
\hline Arsenic, total recoverable & $<5.0$ & 1 & & & & 0 & GE & $<5.0$ & 1 & & & 0 & $\overline{G E}$ & $\mu g \Omega$ \\
\hline Barium, total recoverable & 7.9 & 1 & v & & & 0 & GE & 8.2 & 1 & v & & 0 & GE & $\mu g /$ \\
\hline Benzene & $<2.0$ & 1 & & & & 0 & $\mathrm{GE}$ & $<0.50$ & 1 & & & 0 & GE & $\mu g /$ \\
\hline Beryllium, total recoverable & $<0.015$ & 1 & JV & & & 0 & $\overline{\mathrm{GE}}$ & 0.42 & $i$ & $\mathrm{~J}$ & & 0 & GE & $\mu g / L$ \\
\hline Bromochloromethane & & & & & & & & $<0.050$ & 1 & & & 0 & GE & $\mu g \Omega$ \\
\hline Bromodichloromethane & $<2.0$ & 1 & & & & 0 & GE & $<0.050$ & 1 & & & 0 & $\mathrm{GE}$ & $\mu g \Omega$ \\
\hline Bromoform & $<2.0$ & $i$ & & & & 0 & GE & $<0.050$ & 1 & & & 0 & GE & $\mu g \Omega$ \\
\hline Bromomethane & $<2.0$ & 1 & & & & 0 & GE & $<0.10$ & 1 & & & 0 & GE & $\mu g / 2$ \\
\hline Cadmium, total recoverable & $<2.0$ & 1 & $\mathbf{J}$ & & & 0 & GE & $<0.44$ & 1 & $\mathrm{v}$ & & 0 & GE & $\mu g /$ \\
\hline Carbon disulfide & $<5.0$ & 1 & & & & 0 & GE & $<1.0$ & 1 & & & 0 & GE & $\mu g / L$ \\
\hline Carbon tetrachloride & $<2.0$ & 1 & & & & 0 & GE & $<0.050$ & 1 & & & 0 & $\overline{\mathrm{GE}}$ & $\mu \mathrm{g} / \mathrm{L}$ \\
\hline Chlorobenzene & $<2.0$ & 1 & & & & 0 & GE & $<0.050$ & 1 & & & 0 & GE & $\mu g / \mathrm{L}$ \\
\hline Chloroethane & $<2.0$ & 9 & & & & 0 & GE & $<0.10$ & 1 & & & 0 & $\overline{G E}$ & $\mu g / L$ \\
\hline Chloroethene (Vinyl chloride) & $<2.0$ & 1. & & & & 0 & GE & $<0.10$ & 1 & & & 0 & $\overrightarrow{G E}$ & $\mu g /$ \\
\hline Chloroform & $<2.0$ & 1 & & & & 0 & GE & $<0.050$ & 1 & & & 0 & GE & $\mu g \Omega$ \\
\hline Chloromethane & $<2.0$ & 1 & & & & 0 & $\mathrm{GE}$ & $<0.10$ & 1 & & & 0 & GE & $\mu g /$ \\
\hline Chromium, total recoverable & $<4.0$ & 1 & & & & 0 & $\mathrm{GE}$ & $<4.0$ & 1 & & & 0 & $\mathrm{GE}$ & $\mu g^{\prime} L$ \\
\hline Cobalt, total recoverable & $<0.55$ & 1 & $v$ & & & 0 & GE & $<0.31$ & 1 & V & & 0 & $\mathrm{GE}$ & $\mu g h$. \\
\hline Copper, total recoverable & $<1.6$ & 1 & v & & & 0 & GE & $<0.98$ & 1 & v & & 0 & $\mathrm{GE}$ & $\mu g / L$ \\
\hline Dibromochloromethane & $<2.0$ & 1 & & & & 0 & GE & $<0.050$ & 1 & & & 0 & $\mathrm{GE}$ & $\mu g / L$ \\
\hline 1,2-Dibromo-3-chloropropane & $<2.0$ & 1 & & & & 0 & GE & $<0.050$ & 1 & & & 0 & $\mathrm{GE}$ & $\mu g / L$ \\
\hline 1,2-Dibromoethane & $<2.0$ & 1 & & & & 0 & $\mathrm{GE}$ & $<0.050$ & 1 & & & 0 & GE & $\mu g / L$ \\
\hline Dibromomethane & $<2.0$ & 1 & & & & 0 & GE & $<0.050$ & 1 & & & 0 & GE & $\mu g / \mathrm{L}$ \\
\hline 1,2-Dichlorobenzene & & & & & & & & $<0.050$ & 1 & & & 0 & GE & $\mu g / L$ \\
\hline 1,4-Dichlorobenzene & & & & & & & & $<0.050$ & 1 & & & 0 & GE & $\mu g / \mathrm{L}$ \\
\hline trans-1,4-Dichloro-2-butene & $<2.0$ & 1 & & & & 0 & GE & $<0.50$ & 1 & & & 0 & $\mathrm{GE}$ & $\mu g / \mathrm{L}$ \\
\hline 1,1-Dichloroethane & $<2.0$ & 1 & & & & 0 & GE & $<0.050$ & $i$ & & & 0 & $\mathrm{GE}$ & $\mu g / L$ \\
\hline 1,2-Dichloroethane & $<2.0$ & 1 & & & & 0 & GE & $<0.050$ & 1 & & & 0 & $\mathrm{GE}$ & Har \\
\hline 1,1-Dichloroethylene & $<2.0$ & 1 & & & & 0 & $\mathrm{GE}$ & $<0.050$ & $i$ & & & 0 & GE & $\mu g / L$ \\
\hline cis-1,2-Dichloroethylene & & & & & & & & $<5.0$ & 1 & & & 0 & GE & $\mu g \Omega$ \\
\hline trans-1,2-Dichloroethylene & $<2.0$ & 1 & & & & 0 & GE & $<0.050$ & 1 & & & 0 & $\mathrm{GE}$ & $\mu g / \mathrm{L}$ \\
\hline Dichloromethane & $<10$ & 1 & & & & 0 & GE & $<3.2$ & 1 & $\mathrm{v}$ & & 0 & GE & $\mu g /$ \\
\hline 1,2-Dichloropropane & $<2.0$ & 1 & & & & 0 & $\mathrm{GE}$ & $<0.050$ & 1 & & & 0 & $\mathrm{GE}$ & $\mu \mathrm{g} / \mathrm{L}$ \\
\hline cis-1,3-Dichloropropene & $<2.0$ & 1. & & & & 0 & GE & $<0.050$ & 1 & & & 0 & GE & $\mu g^{\prime} L$ \\
\hline Ethylbenzene & $<2.0$ & 1 & & & & 0 & GE & $<0.050$ & 1 & & & 0 & GE & $\mu g / 2$ \\
\hline 2-Hexanone & $<10$ & 1 & & & & 0 & GE & $<1.0$ & 1 & & & 0 & GE & $\mu g /$ \\
\hline lodomethane (Methyl lodide) & $<10$ & $i$ & & & & 0 & GE & $<1.0$ & 1 & & & 0 & GE & $\mu g / \mathrm{L}$ \\
\hline Lead, total recoverable & $<5.0$ & 1 & & & & 0 & $\overline{G E}$ & 4.4 & 1 & $\mathbf{J}$ & & 0 & GE & $\mu g^{\prime} \mathrm{L}$ \\
\hline Methyl ethyl ketone & $<5.0$ & 1 & & & & 0 & GE & $<1.0$ & $i$ & & & 0 & GE & $\mu g / \mathrm{L}$ \\
\hline Methyl isobutyl ketone & $<10$ & $i$ & & & & 0 & $\mathrm{GE}$ & $<1.0$ & 1 & & & 0 & GE & Hgl \\
\hline Nickel, total recoverable & $<10$ & 1 & & & & 0 & GE & 1.0 & 1 & $\mathrm{~J}$ & & 0 & GE & $\mu g / L$ \\
\hline & 6.0 & $i$ & $J 1$ & & - & 0 & GE & 5.6 & 1 & J1 & - & 0 & GE & $\mathrm{pH}$ \\
\hline Selenium, total recoverable & $<5.0$ & 1 & & & & 0 & GE & $<5.0$ & 1 & & & 0 & $\mathrm{GE}$ & $\mu g / L$ \\
\hline Silver, total recoverable & $<2.0$ & $i$ & & & & 0 & $\mathrm{GE}$ & $<2.0$ & $i$ & J3 & & 0 & GE & $\mu \mathrm{g} / \mathrm{L}$ \\
\hline Specific conductance & 25 & 1 & & & & 0 & $\mathrm{GE}$ & 29 & 1 & & & 0 & GE & $\mu \mathrm{S} / \mathrm{cm}$ \\
\hline Styrene & $<2.0$ & $i$ & & & & 0 & GE & $<0.050$ & 1 & & & 0 & GE & pgl \\
\hline $1,1,1,2$-Tetrachloroethane & $<2.0$ & 1 & & & & 0 & GE & $<0.050$ & 1 & & & 0 & GE & $\mu g \Omega$ \\
\hline
\end{tabular}

- exceeded holding time.

- = exceeded standard. 
WELL LFW 75D (cont.)

Parameter

1,1,2,2-Tetrachloroethane Tetrachloroethylene

Thallium, total recoverable

Toluene

1,1,1-Trichloroethane

1,1,2-Trichloroethane

Trichloroethylene

Trichlorofluoromethane

1,2,3-Trichloropropane

Vanadium, total recoverable

Vinyl acetate

Xylenes

Zinc, total recoverable
1096

$<2.0$

$<2.0$

$<2.0$

$<2.0$

$<2.0$

$<2.0$

$<2.0$

$<2.0$

$<0.66$

$<10$

$<2.0$

43

\section{D}

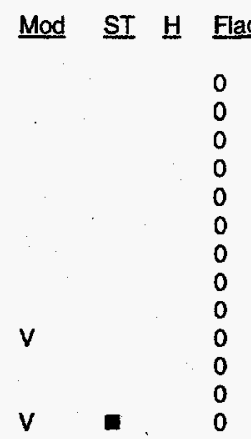

3Q96

$<0.050$

$<0.050$

$<5.0$

$<0.28$

$<0.050$

$<0.050$

$<0.050$

$<0.50$

$<0.050$

$<0.27$

$<1.0$

$<0.15$

28
DF Mod ST H Flag Lab : Unit

V

GE $\mu g h$

$\mu g /$

$\mu g /$

$\mu g h$

$\mu \mathrm{gl}$

ughl

$\mu g / L$

$\mu g / L$

$\mu \mathrm{g} / \mathrm{L}$

$\mu g / L$

$\mu g h$

$\mu g / L$

$\mu g / L$

- = exceeded holding time.

- = exceeded standard. 
THIS PAGE LEFT BLANK INTENTIONALLY. 
WSRC-TR-96-0186-4

Unclassified

\section{Appendix E}

Data Quality/Usability Assessment 
WSRC-TR-96-0186-4

Unclassified

THIS PAGE LEFT BLANK INTENTIONALLY. 


\section{Data Quality/Usability Assessment}

Quality assurance/quality control (QAQC) procedures relating to accuracy and precision of analyses performed on groundwater samples are followed in the field and laboratory, and QC data are reviewed prior to publication of results. The review by the Environmental Protection Department' Environmental Monitoring Section (EPD/EMS) of the volume of analytical data acquired each quarter and presented in various reports is an ongoing process; EPD/EMS' review of the QC data cannot be completed in time to meet the deadlines for the groundwater monitoring reports required by the Resource Conservation and Recovery Act and associated regulations. Beginning first quarter 1996, the Quality Control Samples section of EMS' quarterly groundwater monitoring report contains detailed evaluation of the following indicators of data quality: precision, accuracy, representativeness, comparability, and completeness. Site and regulatory personnel can obtain further information on the data quality and usability in a variety of ways, including those described below.

\section{Data Qualification}

The contract laboratories continually assess their own accuracy and precision according to U.S. Environmental Protection Agency (EPA) guidelines. They submit sample- or batch-specific QC information either at the same time as analytical results or in quarterly summaries. Properly defined and used modifiers (also referred to as qualifiers) can be a key component in assessing data usability. Modifiers designed by EPA or EPD/EMS and used by the primary laboratories or EMS' data validators are presented in Appendix $D$.

\section{Assessment of Accuracy of the Data}

Accuracy, or the nearness of the reported result to the true concentration of a constituent in a sample, can be assessed in several ways.

A laboratory's general accuracy can be judged by evaluation of results obtained from known samples. The contract laboratories analyze commercial reference samples periodically at EPD/EMS' request. The results of these analyses from the nonradionuclide laboratories are presented in the EPD/EMS groundwater monitoring quarterly reports; the results from the radionuclide laboratories should be available on request from the laboratory subcontract representatives. The primary laboratories also seek or maintain state certification by participating periodically in performance studies. Reference samples and evaluation of results are provided by EPA. Results of these studies also are published in the EPD/EMS quarterly reports.

Analysis of blanks provides a tool for assessing the accuracy of both sampling and laboratory analysis. Results for all field blanks for the quarter can be found in the EPD/EMS quarterly reports. Any field or laboratory blanks that exceed established minimums are identified in the same reports, in tables associating them with groundwater samples analyzed in the same batches. In these regulatory reports, the modifier $V$ is assigned to every detected result that is run in the same batch as a laboratory blank with positive results for that analyte. A $U$ qualifier (reported as less than $[<]$ ) also is added if the analyte concentration is less than five times the concentration in the blank (or, for common laboratory contaminants, less than ten times).

Surrogates, organic compounds similar in chemical behavior to the compounds of interest but not normally found in environmental samples, are used to monitor the effect of the matrix on the accuracy of analyses for organic parameters. For example, for analyses of volatile organics by EPA Method 8240, three surrogate compounds are added to all samples and blanks in each analytical batch. In analyses of semivolatile organics by EPA Method 8270 , three surrogates are required and an additional compound is advised for each fraction (acids and base/neutrals). Two surrogates are used in organochlorine pesticides analyses. Percent recoveries for surrogate analyses are calculated by laboratory personnel, reported to EPD/EMS, reviewed, and entered into the database. 
Beginning first quarter 1996, statistical summaries are published in the Quality Control Samples section of the EPD/EMS quarterly groundwater monitoring report. If recoveries are not within specified limits, the laboratory is expected to reanalyze the samples or attach qualifiers to the data identifying the anomalous results.

Sample-specific accuracy for both organic and inorganic parameters can be assessed by examination of matrix spike/matrix spike duplicate results. A portion of the sample is analyzed unspiked to determine a baseline set of values. A second portion of the sample is spiked with known concentrations of compounds appropriate to the analyses being performed, typically five volatile organic compounds for volatile organics analyses, eleven semivolatile compounds for semivolatiles, six pesticide compounds for pesticides, all metals for metals analyses by SW-846 methods (EPA, 1986), and a known quantity of cyanide for cyanide analysis. The percentage of the spike compound that is recovered (i.e., measured in excess of the value obtained for the unspiked sample) is a direct measure of analytical accuracy. EPA requires matrix spike/rnatrix spike duplicates to be run at least once per 20 samples of similar matrix.

Matrix spike/matrix spike duplicate results are reported to EPD/EMS. Beginning first quarter 1996, spike results and a statistical summary are published in the Quality Control Samples section of the EPD/EMS quarterly groundwater monitoring report. For organic compounds, according to EPA guidelines, no action is taken on the basis of matrix spike/matrix spike duplicate data alone (i.e., no result modifiers are assigned solely on the basis of matrix spike results); however, the results can indicate if a laboratory is having a systematic problem in the analysis of one or more analytes.

In the case of inorganic compounds, such as metals, the matrix spike sample analysis provides information about the effect of each sample matrix on the digestion and measurement methodology. Data qualifiers assigned by the laboratories on the basis of the percentage of spike recovery are reported in the EPD/EMS results tables.

\section{Assessment of Precision}

Precision of the analyses, or agreement of a set of replicate results among themselves, is assessed through the use of duplicates initiated by the laboratory and blind replicates provided by EPD/EMS. The results of duplicate and replicate analyses are presented in results tables that report only one quarter of data. Results tables that include more than one quarter of data present only the highest result for each analyte for each quarter of the year.

The laboratories assess precision by calculating the relative percent difference (RPD) for each pair of laboratory-initiated duplicate results. The EMS data validators apply a data qualifier to the results of entire preparation batches of metals analyses when the FPD for laboratory duplicates is greater than 20 percent and either the difference between the two samples is greater than the contractrequired detection limit (CRDL) or both sample results are greater than 5 times the CRDL. This qualifier is published in the EPD/EMS quarterly reports.

Additional statistical comparisons of laboratory duplicate and blind replicate results, both intra- and interlaboratory, are presented in the EPD/EMS quarterly reports. The calculation used for these reports is the mean relative difference (MRD), which is similar to EPA's RPD except that the MRD is the average of all the RPD values from one laboratory for each compound (intralaboratory MRD) or all the RPD values from all laboratories for each compound (interlaboratory MRD) during one quarter. Because detection limits may vary among samples, the MRD requires calculation of a reference detection limit, which is the detection limit at the 90th percentile of the array of limits in the population of all duplicate and replicate analyses for a given analyte during a particular quarter. The MRD is not method-specific.

\section{Method-Specific Accuracy and Precision}

The contract laboratories' EPA-approved laboratory procedures include QAVC requirements as an integral part of the methods. Thus, knowledge of the method used in obtaining data is an important 
component of determining data usability. EPA has conducted extensive research and development on the methods approved for the analysis of water and wastewater. Information on the accuracy and precision of a method is available from EPA publications, as is full information on required QAVQC procedures. A listing of the methods used by the primary laboratories during fourth quarter 1995 is given below, along with the source for the method description. Many, if not all, of these sources include presentations of representative accuracy and precision results. The EPD/EMS quarterly reports provide the methods used by the laboratories each quarter.

\section{Table E-1. Methods Used by the Contract Laboratories}

Method
EICHROMTC
EMLAM01MOD
EMLPU02MOD
EMLSR02MOD
EMLTH01MOD
EMLU02MOD
ENICMOD
EPA120.1
EPA150.1
EPA160.1
EPA160.2
EPA180.1
EPA200.7
EPA245.1
EPA300.0
EPA310.1
EPA335.2
EPA335.3
EPA340.2
EPA350.1
EPA350.3
EPA353.1
EPA353.2
EPA365.2
EPA365.3
EPA365.4
EPA05.1/SM

\section{Used to Analyze}

Technetium isotopes

Americium, curium isotopes

Neptunium, plutonium isotopes

Strontium isotopes

Thorium isotopes

Uranium isotopes

Carbon-14

Specific conductance

$\mathrm{pH}$

Total dissolved solids

Total dissolved solids, total suspended solids

Turbidity

Metals

Mercury

Chloride, nitrite, sulfate, fluoride

Alkalinity (as $\mathrm{CaCO}_{3}$ )

Cyanide

Cyanide

Fluoride

Ammonia nitrogen

Ammonia

Nitrogen, nitrate-nitrite

Nitrogen, nitrate, nitrite, or combined

Phosphorus, all forms (reported as total phosphates)

Phosphorus, all forms (reported as total phosphates)

Phosphorus, all forms (reported as total phosphates)

Five-day biochemical oxygen demand

Five-day biochemical oxygen demand

\section{Source}

EiChrom Industries, Inc.; $\mathrm{NA}^{\mathrm{D}}$

DOE, 1992

DOE, 1992

DOE, 1992

DOE, 1992

DOE, 1992

NA

EPA EMSL, 1983

EPA EMSL, 1983

EPA EMSL, 1983

EPA EMSL, 1983

EPA EMSL, 1983

EPA EMSL, 1983

EPA EMSL, 1983

EPA EMSL, 1983

EPA EMSL, 1983

EPA EMSL, 1983

EPA EMSL, 1983

EPA EMSL, 1983

EPA EMSL, 1983

EPA EMSL, 1983

EPA EMSL, 1983

EPA EMSL, 1983

EPA EMSL, 1983

EPA EMSL, 1983

EPA EMSL, 1983

EPA EMSL, 1983

EPA EMSL, 1983 
Method

EPA410.4

EPA415.1

EPA418.1

EPA420.2

EPA450.1

EPA900.0MOD

EPA901.1MOD

EPA903.0MOD

EPA904.0MOD

EPA906.0MOD

EPA6010

EPA6010A

EPA7470

EPA8080

EPA8150

EPA8240

EPA8260

EPA8270

EPA8280

EPA9020A

EPA9020B

EPA9060

EPIA-001 ${ }^{\mathrm{C}}$

EPIA-002 ${ }^{\circ}$

EPIA- $003^{\circ}$

EPIA-004

EPIA-005 ${ }^{\circ}$

EPIA-006 ${ }^{\circ}$

EPIA-008

EPIA- $009^{\circ}$

EPIA-010

EPIA-011 ${ }^{\circ}$

EPIA-012 ${ }^{\mathrm{C}}$

EPIA-013 ${ }^{\circ}$

EPIA-022 ${ }^{\circ}$

MMES16009MOD
Used to Analyze

Chemical oxygen demand

Dissolved organic carbon, total inorganic carbon, total organic carbon

Total petroleum hydrocarbons

Phenols

Total organic halogens

Gross alpha, nonvolatile beta

Gamma PHA, iodine isotopes

Total alpha-emitting radium, radium isotopes

Radium isotopes

Tritium

Metals

Metals

Mercury

Organochlorine pesticides and PCBs

Chlorinated herbicides

GCMS volatiles

GCMS volatiles

GCMS semivolatiles

Dioxins and furans

Total organic halogens

Total organic halogens

Total inorganic carbon

Gross alpha, nonvolatile beta

Tritium

Carbon-14

Strontium-90

Technetium-99

lodine-129

Radium-226

Radium-228

Radium, total alpha-emitting

Americium, curium, uranium isotopes

Neptunium, plutonium, thorium isotopes

Gamma PHA

Nickel isotopes

Technetium-99
Source

EPA EMSL, 1983

EPA EMSL, 1983

EPA EMSL, 1983

EPA EMSL, 1983

NA

EPA EMSL, 1980

EPA EMSL, 1980

EPA EMSL, 1980

EPA EMSL, 1980

EPA EMSL, 1980

EPA, 1986

EPA, 1992

EPA, 1986

EPA, 1986

EPA, 1986

EPA, 1986

EPA, 1986

EPA, 1986

EPA, 1986

EPA, 1986

EPA, 1994b

EPA, 1986

EP, 1996

EP, 1996

EP, 1996

EP, 1996

EP, 1996

EP, 1996

EP, 1996

EP, 1996

EP, 1996

EP, 1996

EP, 1996

EP, 1996

EP, 1996

NA 
Method

3Q1-6-1420

\section{Used to Analyze}

Total activity, tritium

\section{Source}

NA

${ }^{a} M O D$ indicates modifications of applicable EPA, DOE, or other procedures.

${ }^{\mathrm{b}} \mathrm{NA}=$ not available. Sources for some proprietary methods are not available.

'The methods and detection limits used by GP are in-house methods based on applicable EPA, DOE, or other procedures. Complete method descriptions are available from GP.

Note: One of the labs reports the method for some metals determinations as CLP or CLP-MOD. This is presumed equivalent to Method 6010 or $6010 \mathrm{~A}$.

An example of method-specific QC information is that for EPA Method 8270 (EPA, 1986), which is used by both $G E$ and Weston for analyses of semivolatile organics. The following table gives method-specific accuracy and precision as functions of concentration. Contract laboratories are expected to achieve or at least approach these limits.

Table E-2. Method Accuracy and Precision as Functions of Concentration for EPA Method 8270

\begin{tabular}{|c|c|c|c|}
\hline Parameter & $\begin{array}{l}\text { Accuracy, as } \\
\text { recovery, } x^{\circ}(\mu g / L)\end{array}$ & $\begin{array}{l}\text { Single analyst } \\
\text { precision, } \mathrm{s}_{\mathrm{r}}^{-}(\mu \mathrm{g} / \mathrm{h})\end{array}$ & $\begin{array}{l}\text { Overall prec } \\
S^{\prime}(\mu g / L)\end{array}$ \\
\hline Acenaphthene & $0.96 C+0.19$ & $0.15 x-0.12$ & $0.21 x-0.67$ \\
\hline Acenaphthylene & $0.89 C+0.74$ & $0.24 X-1.06$ & $0.26 x-0.54$ \\
\hline Aldrin & $0.78 C+1.66$ & $0.27 x-1.28$ & $0.43 X+1.13$ \\
\hline Anthracene & $0.80 C+0.68$ & $0.21 x-0.32$ & $0.27 x-0.64$ \\
\hline Benzo[a]anthracene & $0.88 C-0.60$ & $0.15 X+0.93$ & $0.26 x-0.21$ \\
\hline Benzo[b]fluoranthene & $0.93 C-1.80$ & $0.22 X+0.43$ & $0.29 X+0.96$ \\
\hline Benzo[Kfluoranthene & $0.87 C-1.56$ & $0.19 X+1.03$ & $0.35 X+0.40$ \\
\hline Benzo $[g, h, i]$ perylene & $0.98 C-0.86$ & $0.29 X+2.40$ & $0.51 \times-0.44$ \\
\hline Benzo[a]pyrene & $0.90 C-0.13$ & $0.22 X+0.48$ & $0.32 X+1.35$ \\
\hline$\beta-B H C$ & $0.87 C-0.94$ & $0.20 x-0.58$ & $0.30 X+1.94$ \\
\hline$\delta-\mathrm{BHC}$ & $0.29 C-1.09$ & $0.34 X+0.86$ & $0.93 X+0.17$ \\
\hline Bis(2-chloroethoxy) methane & $1.12 C-5.04$ & $0.16 x+1.34$ & $0.26 x+2.01$ \\
\hline Bis(2-chloroethyl) ether & $0.86 C-1.54$ & $0.35 \times-0.99$ & $0.35 X+0.10$ \\
\hline Bis(2-chloroisopropyl) ether & $1.03 c-2.31$ & $0.24 X+0.28$ & $0.25 X+1.04$ \\
\hline Bis(2-ethylhexyl) phthalate & $0.84 C-1.18$ & $0.26 X+0.73$ & $0.36 X+0.67$ \\
\hline 4-Bromophenyl phenyl ether & $0.91 C-1.34$ & $0.13 X+0.66$ & $0.16 X+0.66$ \\
\hline Butylbenzyl phthalate & $0.66 \mathrm{C}-1.68$ & $0.18 X+0.94$ & $0.53 x+0.92$ \\
\hline 4-Chloro-m-cresol & $0.84 C+0.35$ & $0.23 X+0.75$ & $0.29 x+1.31$ \\
\hline Chloroethane & $0.99 c-1.53$ & $0.14 x-0.13$ & $0.17 x-0.28$ \\
\hline 2-Chloronaphthalene & $0.89 C+0.01$ & $0.07 X+0.52$ & $0.13 x+0.34$ \\
\hline 2-Chlorophenol & $0.78 C+0.29$ & $0.18 X+1.46$ & $0.28 X+0.97$ \\
\hline
\end{tabular}




Parameter
4-Chlorophenyl phenyl ethe
Chrysene
p,p'-DDD
p,p'-DDE
p,p'-DDT
Dibenz[a,h]anthracene
Di-n-butyl phthalate
1,2-Dichlorobenzene
1,3-Dichlorobenzene
1,4-Dichlorobenzene
3,3'-Dichlorobenzidine
2,4-Dichlorophenol
Dieldrin
Diethyl phthalate
2,4-Dimethyl phenol
Dimethyl phthalate
2,4-Dinitrophenol
2,4-Dinitrotoluene
2,6-Dinitrotoluene
Di-n-octyl phthalate
Endosulfan sulfate
Endrin aldehyde
Fluoranthene
Fluorene
Heptachlor
Heptachlor epoxide
Hexachlorobenzene
Hexachlorobutadiene
Hexachloroethane
Indeno[1,2,3-c,d]pyrene
Isophorone
2-Methyl-4,6-dinitrophenol
Naphthalene
Nitrobenzene
2-Nitrophenol

\section{Parameter}

4-Chlorophenyl phenyl ether

Chrysene

Accuracy, as recovery, $x^{\prime}(\mu g / L)$

$0.91 \mathrm{C}+0.53$

$0.93 \mathrm{C}-1.00$

$0.56 \mathrm{C}-0.40$

$0.70 \mathrm{C}-0.54$

$0.79 \mathrm{C}-3.28$

$0.88 \mathrm{C}+4.72$

$0.59 \mathrm{C}+0.71$

$0.80 \mathrm{C}+0.28$

$0.86 \mathrm{C}-0.70$

$0.73 \mathrm{C}-1.47$

$1.23 \mathrm{C}-12.65$

$0.87 \mathrm{C}-0.13$

$0.82 \mathrm{C}-0.16$

$0.43 \mathrm{C}+1.00$

$0.71 C+4.41$

$0.20 \mathrm{C}+1.03$

$0.81 \mathrm{C}-18.04$

$0.92 C-4.81$

$1.06 \mathrm{C}-3.60$

$0.76 \mathrm{C}-0.79$

$0.39 \mathrm{C}+0.41$

$0.76 \mathrm{C}-3.86$

$0.81 \mathrm{C}+1.10$

$0.90 \mathrm{C}-0.00$

$0.87 \mathrm{C}-2.97$

$0.92 \mathrm{C}-1.87$

$0.74 \mathrm{C}+0.66$

$0.71 \mathrm{C}-1.01$

$0.73 \mathrm{C}-0.83$

$0.78 \mathrm{C}-3.10$

$1.12 \mathrm{C}+1.41$

1.04C-28.04

$0.76 \mathrm{C}+1.58$

$1.09 \mathrm{C}-3.05$

$0.07 \mathrm{C}-1.15$

$0.61 \mathrm{C}-1.22$
Single analyst Overall precision, precision, $\mathbf{s}_{\mathbf{r}}{ }^{\prime}(\mu g / L) \quad \mathbf{S}^{\prime}(\mu g / L)$

$0.20 x-0.94$

$0.28 x+0.13$

$0.29 \times-0.32$

$0.26 \times-1.17$

$0.42 x+0.19$

$0.30 x+8.51$

$0.13 x+1.16$

$0.20 x+0.47$

$0.25 x+0.68$

$0.24 X+0.23$

$0.28 x+7.33$

$0.15 x+1.25$

$0.20 x-0.16$

$0.28 x+1.44$

$0.16 X+1.21$

$0.54 x+0.19$

$0.38 X+2.36$

$0.12 x+1.06$

$0.14 X+1.26$

$0.21 x+1.19$

$0.12 x+2.47$

$0.18 x+3.91$

$0.22 x-0.73$

$0.12 X+0.26$

$0.24 x-0.56$

$0.33 x-0.46$

$0.18 x-0.10$

$0.19 \chi+0.92$

$0.17 X+0.67$

$0.29 X+1.46$

$0.27 x+0.77$

$0.10 X+42.29$

$0.21 x-0.41$

$0.19 X+0.92$

$0.16 X+1.94$

$0.38 X+2.57$
$0.30 X-0.46$

$0.33 X-0.09$

$0.66 X-0.96$

$0.39 X-1.04$

$0.65 \times-0.58$

$0.59 X+0.25$

$0.39 X+0.60$

$0.24 X+0.39$

$0.41 X+0.11$

$0.29 X+0.36$

$0.47 X+3.45$

$0.21 X+1.28$

$0.26 x-0.07$

$0.52 X+0.22$

$0.22 X+1.31$

$1.05 x-0.92$

$0.42 X+26.29$

$0.21 X+1.50$

$0.19 X+0.35$

$0.37 X+1.19$

$0.63 X-1.03$

$0.73 X-0.62$

$0.28 X-0.60$

$0.13 X+0.61$

$0.50 X-0.23$

$0.28 X+0.64$

$0.43 X-0.52$

$0.26 X+0.49$

$0.17 X+0.80$

$0.50 X-0.44$

$0.33 X+0.26$

$0.26 X+23.10$

$0.30 x-0.68$

$0.27 X+0.21$

$0.27 X+2.60$

$0.44 X+3.24$ 
Parameter

N-Nitrosodi-n-propylamine

PCB 1260

Pentachlorophenol

Phenanthrene

Phenol

Pyrene

1,2,4-Trichlorobenzene

2,4,6-Trichlorophenol
Accuracy, as recovery, $x^{\prime}(\mu g / L)$

Single analyst precision, $s_{r}^{\prime}(\mu g / L) \quad S^{\prime}(\mu g / L)$

$1.12 \mathrm{C}-6.22$

$0.81 \mathrm{C}-10.86$

$0.93 C+1.99$

$0.87 \mathrm{C}+0.06$

$0.43 C+1.26$

$0.84 \mathrm{C}-0.16$

$0.94 \mathrm{C}-0.79$

$0.91 \mathrm{C}-0.18$
$0.27 X+0.68$

$0.35 X+3.61$

$0.24 X+3.03$

$0.12 X+0.57$

$0.26 X+0.73$

$0.16 X+0.06$

$0.15 X+0.85$

$0.16 X+2.22$
Overall precision, $0.44 X+0.47$

$0.43 X+1.82$

$0.30 X+4.33$

$0.15 X+0.25$

$0.35 X+0.58$

$0.15 X+0.31$

$0.21 X+0.39$

$0.22 X+1.81$

$x^{\prime}=$ Expected recovery for one or more measurements of a sample containing a concentration of $C$, in $\mu g / L$.

$s_{r}{ }^{\prime}=$ Expected single analyst standard deviation of measurements at an average concentration of $X$, in $\mu g / L$.

$S^{\prime}=$ Expected interlaboratory standard deviation of measurements at an average concentration of $X$, in $\mu g / L$.

$\mathrm{C}=$ True value for the concentration, in $\mu \mathrm{g} / \mathrm{L}$.

$X=$ Average recovery found for measurements of samples containing a concentration of $\mathrm{C}$, in $\mu \mathrm{g} / \mathrm{L}$. 
WSRC-TR-96-0186-4

Unclassifjed

THIS PAGE LEFT BLANK INTENTIONALLY. 


\section{Appendix F}

Groundwater Monitoring Results for Individual Wells for Special Study 
WSRC-TR-96-0186-4

Unclassified

THIS PAGE LEFT BLANK INTENTIONALLY. 


\section{Groundwater Monitoring Results for Individual Wells for Special Study}

In addition to regularly scheduled first quarter and third quarter sampling events, several wells at the Interim Sanitary Landfill were sampled for special investigation during 1996. In January, wells LFW 32 and 74D were sampled to investigate elevated concentrations of volatile organic constituents noted during third quarter 1995. In April, four wells that monitor groundwater at the Interim Sanitary Landfill, LFW 32, 43C, 43D, and 74D, and four additional wells in the LFW series, LFW 30, 31, 33, and 34, were sampled and analyzed for Appendix II constituents as required by South Carolina Solid and Hazardous Waste Regulation R61-107.258. 
Table F-1. Groundwater Monitoring Results for Individual Wells for the Special Study, First Quarter 1996

WELL LFW 32

\begin{tabular}{|c|c|c|c|c|c|c|}
\hline SRS Coord. & Lathongitude & Screen Zone Elevation & Ton of Casing & Casing & Pump & Screen Zone \\
\hline $\begin{array}{l}N 85836.8 \\
E 44935.9\end{array}$ & $\begin{array}{l}33.288920^{\circ} \mathrm{N} \\
81.715210^{\circ} \mathrm{W}\end{array}$ & $165.3-144.3 \mathrm{ft} \mathrm{msl}$ & $223.7 \mathrm{ft} \mathrm{msl}$ & 4" PVC & $\mathbf{S}$ & Upper Steed Pond \\
\hline \multicolumn{7}{|l|}{ FIELD DATA } \\
\hline Parameter & 1096 & & Unit & & & \\
\hline $\begin{array}{l}\text { Water elevation } \\
\text { pH } \\
\text { Sp. conductance } \\
\text { Water temperature } \\
\text { Alkalinity as } \mathrm{CaCO}_{3} \\
\text { Turbidity } \\
\text { Volume purged }\end{array}$ & $\begin{array}{l}162.7 \\
4.6 \\
22 \\
23.0 \\
3 \\
0 \\
7.5\end{array}$ & & $\begin{array}{l}\text { ft msl } \\
\mathrm{pH} \\
\mu \mathrm{S} / \mathrm{cm} \\
{ }^{\circ} \mathrm{C} \\
\mathrm{mg} / \mathrm{L} \\
\mathrm{NTU} \\
\text { well vol }\end{array}$ & & & \\
\hline
\end{tabular}

ANALYTICAL DATA

Parameter

Acetone

Acetonitrile (Methyl cyanide)

Acrolein

Acrylonitrile

Allyl chloride

Benzene

Bis(2-chloroisopropyl) ether

Bromodichloromethane

Bromoform

Bromomethane

Carbon disulfide

Carbon tetrachloride

Chlorobenzene

Chloroethane

Chloroethene (Vinyl chloride)

Chloroform

Chloromethane

Chloroprene

Dibromochloromethane

1,2-Dibromo-3-chloropropane

1,2-Dibromoethane

Dibromomethane

trans-1,4-Dichloro-2-butene

Dichlorodifluoromethane

1,1-Dichloroethane

1,2-Dichloroethane

1,1-Dichloroethylene

trans-1,2-Dichloroethylene

Dichloromethane

1,2-Dichloropropane

cis-1,3-Dichloropropene

trans-1,3-Dichloropropene

Ethylbenzene

2-Hexanone

lodomethane (Methyl iodide)

Isobutyl alcoho'

Methacrylonitrile

Methyl ethyl ketone

Methyl isobutyl ketone

Methyi methacrylate

Propionitrile

Styrene

$1,1,1,2-$ Tetrachloroethane

1,1,2,2-Tetrachloroethane

Tetrachloroethylene

Toluene

1,1,1-Trichloroethane
$<10.0$

$<10.0$

$<20.0$

$<50.0$

$<10.0$

$<2.00$

$<20.0$

$<2.00$

$<2.00$

$<2.00$

$<5.00$

$<2.00$

$<2.00$

$<2.00$

$<2.00$

$<2.00$

$<2.00$

$<2,00$

$<2.00$

$<2.00$

$<200$

$<2.00$

$<2.00$

1.06

$<2.00$

$<2.00$

$<2.00$

$<2.00$

$<1.36$

$<2.00$

$\infty .00$

$<2.00$

$<2.00$

$<10.0$

$<10.0$

$<20.0$

$<10.0$

$<5.00$

$<10.0$

$<10.0$

80.

$<2.00$

$<2.00$

$<2.00$

$<2.00$

$<2.00$

7.9

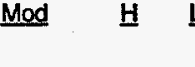

Lab Unit

GE $\mu g h$

GE $\mu g / 2$

GE $\mu g / L$

GE $\mu g /$

GE $\mu g /$ h

GE $\mu g /$

GE $\mu g \Omega$

GE $\mu g /$

GE $\mu g /$

GE $\mu g /$

GE $\mu g /$

GE $\quad \mu g /$.

GE $\mu g /$.

GE $\mu g /$

GE $\mu g /$ L

GE $\mu g / L$

GE $\mu g / L$

JE GE $\mu g /$ h

GE $\mu g / L$

GE $\mu g /$ L

GE $\mu g /$ L

V

$\mu g / L$
$\mu g / L$

$\mu g /$

$\mu g / 2$

$\mu g h$

$\mu g /$.

g/l

$\mu g / L$

$\mu g /$.

$\mu g / 2$

$\mu g h$

$\mu g / 2$

$\mu g / 2$

$\mu g / L$

$\mu g / L$

$\mu g / L$

$\mu \mathrm{g} / \mathrm{L}$

ugl

$\mu g /$

$\mu g / 2$

Note: Modifiers are listed and detailed in Appendix D, Key to Reading the Tables. $\bullet=$ exceeded holding time. 
Well LFW 32 (cont.)

Parameter

1096

DF $\quad$ Mod

1,1,2-Trichloroethane

Trichloroethylene

$<2.00$

1

Trichlorofluoromethane

$<2.00$

95.5

1,2,3-Trichloropropane

$<2.00$

Vinyl acetate

Xylenes

$<2.00$

H Lab Unit

GE $\mu g / L$

GE $\mu g / 2$

GE $\mu g / L$

GE $\mu g / L$

GE

$\mu g / L$

Note: Modifiers are listed and detailed in Appendix D, Key to Reading the Tables. $\bullet=$ exceeded holding time. 
WSRC-TR-96-0186-4

Unclassified

WELL LFW 74D

\begin{tabular}{lc} 
SRS Coord & Lat/Longitude \\
\hline N85828.1 & $33.289172^{\circ} \mathrm{N}$ \\
E45098.0 & $81.714771^{\circ} \mathrm{W}$ \\
SAMPLE DATE & $01 / 15 / 96$
\end{tabular}

Screen Zone Elevation

$167.7 \cdot 152.7 \mathrm{ft} \mathrm{msl}$

$\begin{array}{lll}\text { Top of Casing } & \text { Casing } & \text { Pump } \\ 213.9 \mathrm{ft} \mathrm{ms} & 4^{*} \text { PVC } & \mathrm{S}\end{array}$

Unit

ft msl

pH

$\mu \mathrm{S} / \mathrm{cm}$

mgh

well vol

Turbidity

Volume purged

Sampling code

162.6
5
16
24.0
1
1
6.9

ANALYTICAL DATA

Parameter

Acetone

Acetonitrile (Methyl cyanide)

Acrolein

Acrylonitrile

Allyl chloride

Benzene

Bis(2-chloroisopropyl) ether

Bromodichloromethane

Bromoform

Bromomethane

Carbon disulfide

Carbon tetrachloride

Chlorobenzene

Chloroethane

Chloroethene (Vinyl chloride)

Chloroform

Chloromethane

Chloroprene

Dibromochloromethane

1,2-Dibromo-3-chloropropane

1,2-Dibromoethane

Dibromomethane

trans-1,4-Dichloro-2-butene

Dichlorodifluoromethane

1,1-Dichloroethane

1,2-Dichloroethane

1,1-Dichloroethylene

trans-1,2-Dichloroethylene

Dichloromethane

1,2-Dichloropropane

cis-1,3-Dichloropropene

trans-1,3-Dichloropropene

Ethylbenzene

2-Hexanone

lodomethane (Methyl iodide)

Isobutyl alcohol

Methacrylonitrile

Methyl ethyl ketone

Methyl isobutyl ketone

Methyl methacrylate

Propionitrile

Styrene

$1,1,1,2-$ Tetrachloroethane

1,1,2,2-Tetrachloroethane

Tetrachloroethylene

Toluene

1,1,1-Trichloroethane

1,1,2-Trichloroethane

Trichloroethylene

Trichlorofluoromethane

1,2,3-Trichloropropane

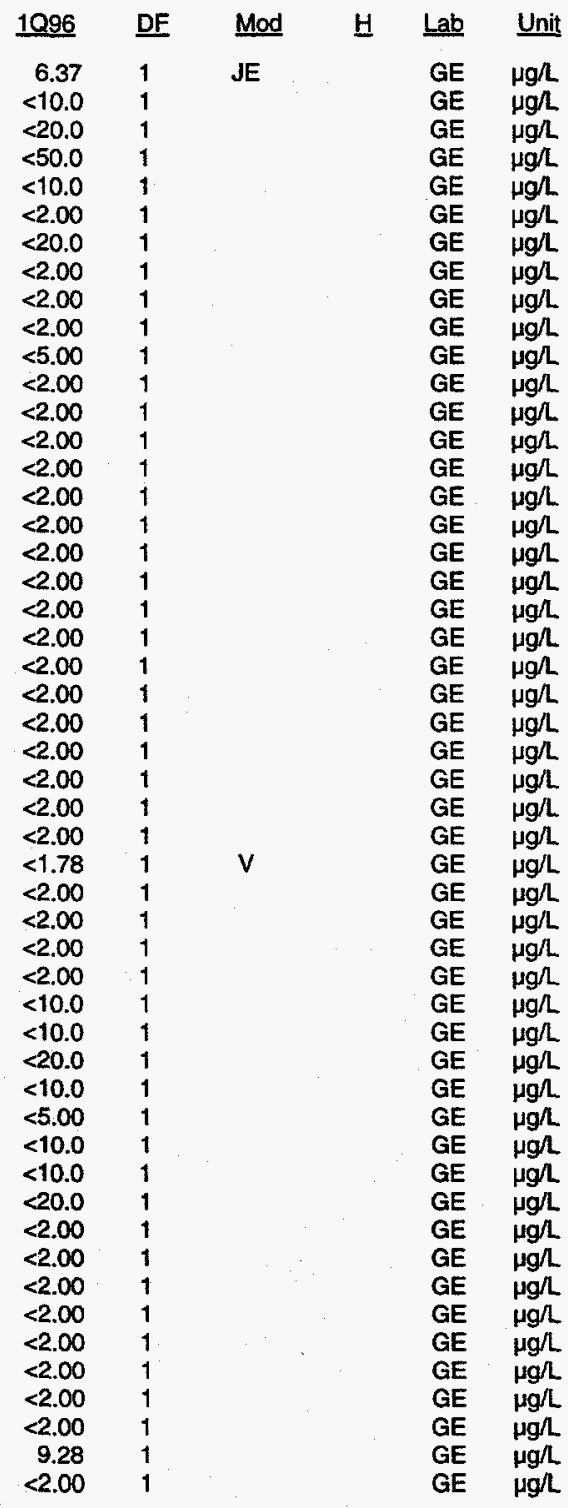

Note: Modifiers are listed and detailed in Appendix D, Key to Reading the Tables.

$\bullet=$ exceeded holding time. 
Well LFW 74D (cont.)

Parameter

Vinyl acetate

Xylenes
1096

$<10.0$ $<2.00$

\begin{tabular}{|c|c|c|}
\hline Mod & $\underline{H}$ & $\underline{\text { Lab }}$ \\
\hline & & $\begin{array}{l}\mathrm{GE} \\
\mathrm{GE}\end{array}$ \\
\hline
\end{tabular}

Note: Modifiers are listed and detailed in Appendix D, Key to Reading the Tables.

- = exceeded holding time. 
Table F-2. Groundwater Monitoring Results for Individual Wells for the Special Study, Second Quarter 1996

\section{WELL LFW 30}

\begin{tabular}{|c|c|c|c|c|c|c|}
\hline SRS Coord. & Lat/Longitude & Screen Zone Elevation & Top of Casing & Casing & Pump & Screen Zone \\
\hline $\begin{array}{l}\text { N86318.4 } \\
\text { E45170.9 }\end{array}$ & $\begin{array}{l}33.290370^{\circ} \mathrm{N} \\
81.715530^{\circ} \mathrm{W}\end{array}$ & $162.7-141.7 \mathrm{ft} \mathrm{msl}$ & $210 \mathrm{ft}$ msl & 4" PVC & $S$ & Upper Steed Pond \\
\hline SAMPLE DATE & $04 / 22 / 96$ & & & & & \\
\hline FIELD DATA & & & & & & \\
\hline Parameter & $\underline{2 Q 96}$ & & $\underline{\text { Unit }}$ & & & \\
\hline $\begin{array}{l}\text { Water elevation } \\
\text { pH } \\
\text { Sp. conductance } \\
\text { Water temperature } \\
\text { Alkalinity as } \mathrm{CaCO}_{3} \\
\text { Turbidity } \\
\text { Volume purged } \\
\text { Sampling code }\end{array}$ & $\begin{array}{l}165.4 \\
5.0 \\
18 \\
25.0 \\
1 \\
0 \\
4.9\end{array}$ & & $\begin{array}{l}\mathrm{ft} \text { msl } \\
\mathrm{pH} \\
\mu \mathrm{S} / \mathrm{cm} \\
{ }^{\circ} \mathrm{C} \\
\mathrm{mg} / \mathrm{L} \\
\mathrm{NTU} \\
\text { well vol }\end{array}$ & & & \\
\hline
\end{tabular}

ANALYTICAL DATA

\begin{tabular}{|c|c|c|c|c|c|}
\hline Parameter & 2096 & $\underline{D F}$ & Mod & $\underline{\mathrm{Lab}}$ & Unit \\
\hline Benzene & $<2.00$ & 1 & & GE & $\mu g / L$ \\
\hline Benzene & $<5.00$ & 1 & & WA & ugh \\
\hline Benzene & $<5.00$ & 1 & & WA & $\mu \mathrm{g} / \mathrm{L}$ \\
\hline Bromodichloromethane & $<2.00$ & 1 & & GE & $\mu g / L$ \\
\hline Bromodichloromethane & $<5.00$ & 1 & & WA & $\mu \mathrm{g} / \mathrm{L}$ \\
\hline Bromodichloromethane & $<5.00$ & 1 & & WA & $\mu g / 2$ \\
\hline Bromodichloromethane & $<5.00$ & 1 & & WA & $\mu g /$ \\
\hline Bromoform & $<2.00$ & 1 & & GE & $\mu g / 2$ \\
\hline Bromoform & $<5.00$ & 1 & & WA & $\mu g /$ \\
\hline Bromoform & $<5.00$ & 1 & & WA & ugh \\
\hline Bromoform & $<5.00$ & 1 & & WA & $\mu g / 2$ \\
\hline Bromomethane & $<2.00$ & 1 & & GE & $\mu g /$ \\
\hline Bromomethane & $<10.0$ & 1 & & WA & $\mu g / L$ \\
\hline Bromomethane & $<10.0$ & 1 & & WA & $\mu g / L$ \\
\hline Bromomethane & $<10.0$ & 1 & & WA & $\mu g / 2$ \\
\hline Carbon tetrachloride & $<2.00$ & 1 & & GE & $\mu g / L$ \\
\hline Carbon tetrachloride & $<5.00$ & 1 & & WA & $\mu g /$ \\
\hline Carbon tetrachloride & $<5.00$ & 1 & & WA & Hg/ \\
\hline Carbon tetrachloride & $<5.00$ & 1 & & WA & $\mu g /$ \\
\hline Chlorobenzene & $<2.00$ & 1 & & GE & $\mu g / h$ \\
\hline Chlorobenzene & $<5.00$ & 1 & & WA & $\mu g / L$ \\
\hline Chlorabenzene & $<5.00$ & 1 & & WA & $\mu g^{\prime} /$ \\
\hline Chloroethane & $<2.00$ & 1 & & GE & $\mu g / L$ \\
\hline Chloroethane & $<10.0$ & 1 & & WA & Ho/L \\
\hline Chloroethane & $<10.0$ & 1 & & WA & $\mu \mathrm{g} / \mathrm{L}$ \\
\hline Chloroethane & $<10.0$ & 1 & & WA & ugl \\
\hline Chloroethene (Vinyl chloride) & $<2.00$ & 1 & & GE & $\mu \mathrm{g} / \mathrm{L}$ \\
\hline Chloroethene (Vinyl chloride) & $<10.0$ & 1 & & WA & $\mu g / 2$ \\
\hline Chloroethene (Vinyl chloride) & $<10.0$ & 1 & & WA & $\mu g / L$ \\
\hline Chloroethene (Vinyl chloride) & $<10.0$ & 1 & & WA & $\mu g / L$ \\
\hline 2-Chloroethyl vinyl ether & $<10.0$ & 1 & & GE & $\mu g h$ \\
\hline 2-Chloroethyl vinyl ether & $<10.0$ & 1 & & WA & $\mu g / L$ \\
\hline 2-Chloroethyl vinyl ether & $<10.0$ & 1 & & WA & $\mu g h$ \\
\hline 2-Chloroethyl vinyl ether & $<10.0$ & 1 & & WA & $\mu \mathrm{g}$ \\
\hline Chloroform & $<2.00$ & 1 & & GE & $\mu g$ \\
\hline Chloroform & $<5.00$ & 1 & & WA & \\
\hline Chloroform & $<5.00$ & 1 & & WA & $\mu g / L$ \\
\hline Chloroform & $<5.00$ & 1 & & WA & $\mu \mathrm{g} / \mathrm{L}$ \\
\hline Chloromethane & $<2.00$ & 1 & & GE & $\mu g / L$ \\
\hline Chloromethane & $<10.0$ & 1 & & WA & \\
\hline Chloromethane & $<10.0$ & 1 & & WA & \\
\hline Chloromethane & $<10.0$ & 1 & & WA & \\
\hline Dibromochloromethane & $<2.00$ & 1 & & GE & \\
\hline Dibromochloromethane & $<5.00$ & 1 & & WA & \\
\hline Dibromochloromethane & $<5.00$ & 1 & & WA & \\
\hline
\end{tabular}

Note: Modifiers are listed and detailed in Appendix D, Key to Reading the Tables.

$\bullet=$ exceeded holding time. 
Well LFW 30 (cont.)

\section{Parameter}

Dibromochloromethane

1,1-Dichloroethane

1,1-Dichloroethane

1,1-Dichloroethane

1,1-Dichloroethane

1,2-Dichloroethane

1,2-Dichloroethane

1,2-Dichloroethane

1,2-Dichloroethane

1,1-Dichloroethylene

1,1-Dichloroethylene

1,1-Dichloroethylene

1,2-Dichloroethylene

1,2-Dichloroethylene

1,2-Dichloroethylene

trans-1,2-Dichloroethylene

Dichloromethane

Dichloromethane

Dichloromethane

Dichloromethane

1,2-Dichloropropane

1,2-Dichloropropane

1,2-Dichloropropane

1.2-Dichloropropane

cis-1,3-Dichloropropene

cis-1,3-Dichloropropene

cis-1,3-Dichloropropene

cis-1,3-Dichloropropene

trans-1,3-Dichloropropene

trans-1,3-Dichloropropene

trans-1,3-Dichloropropene

trans-1,3-Dichloropropene

Ethylbenzene

Ethylbenzene

Ethylbenzene

Ethylbenzene

1,1,2,2-Tetrachloroethane

1,1,2,2-Tetrachloroethane

$1,1,2,2-$ Tetrachloroethane

$1,1,2,2-$ Tetrachloroethane

Tetrachloroethylene

Tetrachloroethylene

Tetrachloroethylene

Tetrachloroethylene

Toluene

Toluene

Toluene

$1,1,1$-Trichloroethane

$1,1,1$-Trichloroethane

1,1,1-Trichloroethane

1,1,1-Trichloroethane

1,1,2-Trichloroethane

1,1,2-Trichloroethane

1,1,2-Trichloroethane

1,1,2-Trichloroethane

Trichloroethylene

Trichloroethylene

Trichloroethylene

Trichlorofluoromethane

Trichlorofluoromethane

Trichlorofluoromethane

Trichlorofluoromethane

Xylenes

Xylenes

Xylenes

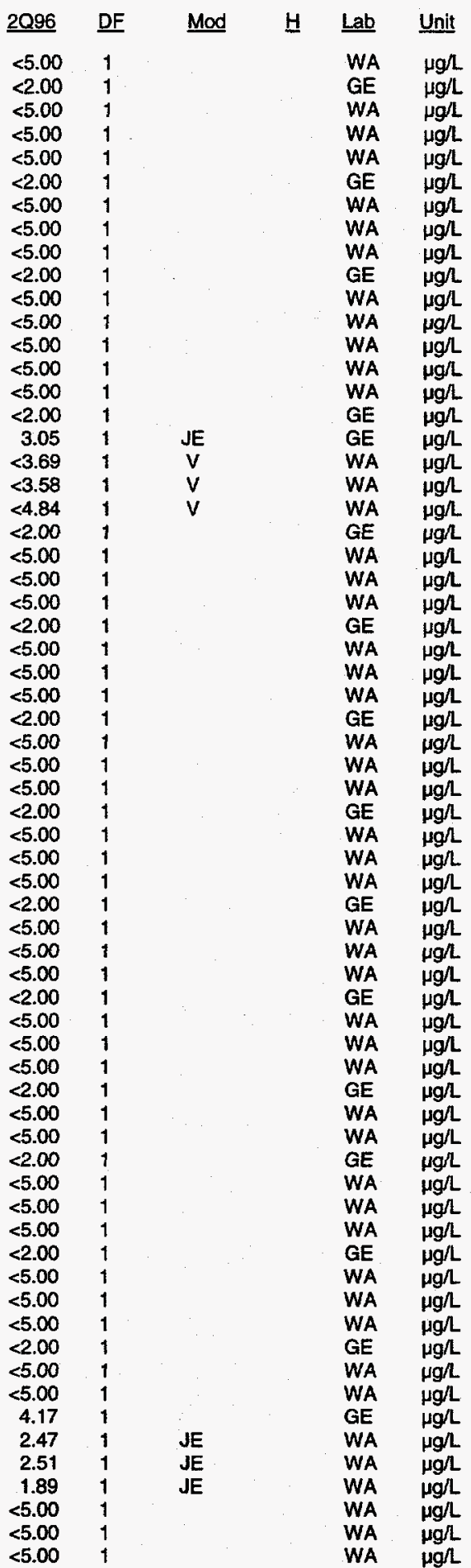

Note: Modifiers are listed and detailed in Appendix D, Key to Reading the Tables.

$\bullet$ = exceeded holding time. 
WELL LFW 30 Replicate

\begin{tabular}{lc} 
SRS Coord. & Lat/Longitude \\
\cline { 2 - 2 } N86318.4 & $33.290370^{\circ} \mathrm{N}$ \\
E45170.9 & $81.715530^{\circ} \mathrm{W}$ \\
SAMPLE DATE & $04 / 229$ \\
FIELD DATA & \\
Parameter & \\
\hline Water elevation & 2096 \\
pH & \\
Sp. conductance & 165.4 \\
Water temperature & 5.0 \\
Alkalinity as CaCO & 18 \\
Turbidity & 25.0 \\
Volume purged & 1 \\
Sampling code & 0 \\
\hline
\end{tabular}

4/22/96

2096

Unit

ft msl

$\mathrm{pH}$

$\mathrm{S} / \mathrm{cm}$

${ }^{\circ} \mathrm{C}$

mgh

well vo

ANALYTICAL DATA

Parameter

2096

$\underline{\mathrm{DF}}$

Mod

Unit

Benzene

Benzene

Bromodichloromethane

Bromoform

Bromoform

Bromomethane

Bromomethane

Carbon tetrachloride

Carbon tetrachloride

Chlorobenzene

Chlorobenzene

Chloroethane

Chloroethane

Chloroethene (Vinyl chloride)

Chloroethene (Vinyl chloride)

2-Chloroethyl vinyl ether

2-Chloroethyl vinyl ether

Chloroform

Chloroform

Chloromethane

Chloromethane

Dibromochloromethane

Dibromochloromethane

1,1-Dichloroethane

1,1-Dichloroethane

1,2-Dichloroethane

1,2-Dichloroethane

1,1-Dichloroethylene

1,1-Dichloroethylene

1,2-Dichloroethylene

trans-1,2-Dichloroethylene

Dichloromethane

Dichloromethane

1,2-Dichloropropane

1,2-Dichloropropane

cis-1,3-Dichloropropene

cis-1,3-Dichloropropene

trans-1,3-Dichloropropene

trans-1,3-Dichloropropene

Ethylbenzene

Ethylbenzene

1,1,2,2-Tetrachloroethane

$1,1,2,2-$ Tetrachloroethane

Tetrachloroethylene

Tetrachloroethylene

Toluene

Toluene

1,1,1-Trichloroethane

1,1,1-Trichloroethane

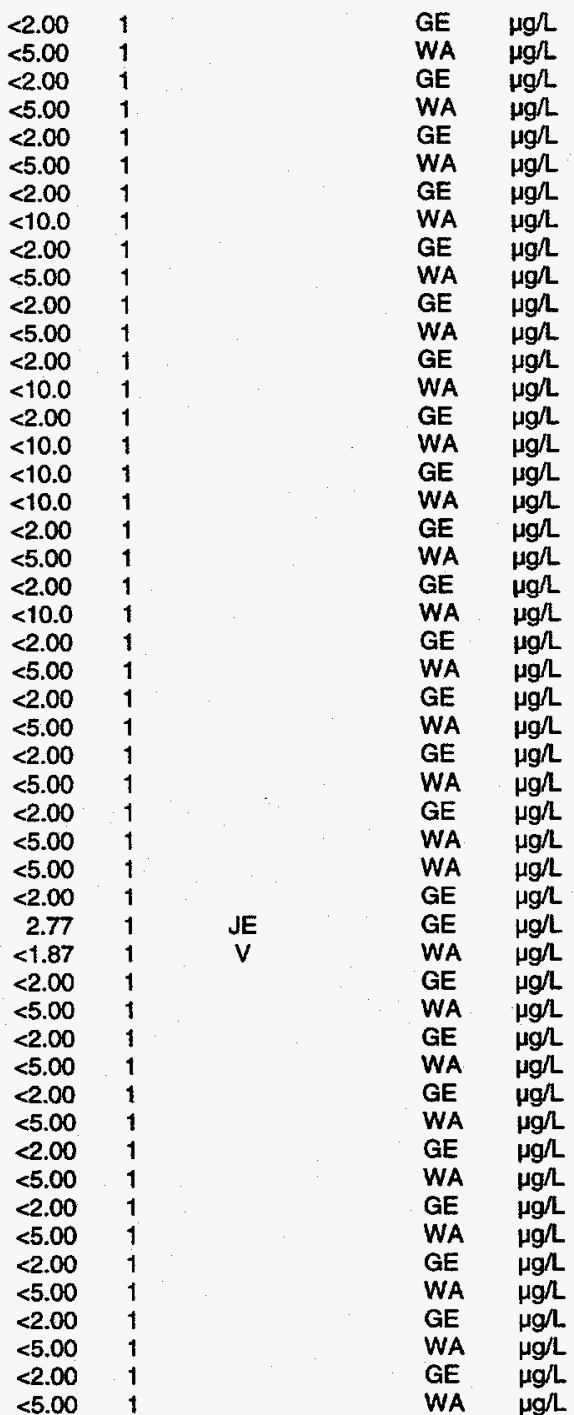

Note: Modifiers are listed and detailed in Appendix D, Key to Reading the Tables. - = exceeded holding time. 
Well LFW 30 Replicate (cont.)

Parameter

1,1,2-Trichloroethane

1,1,2-Trichloroethane

Trichloroethylene

Trichloroethylene

Trichlorofluoromethane

Trichlorofluoromethane

Xylenes

$\begin{array}{cccccc}2096 & \text { DF } & \text { Mod } & \text { 브 } & \text { Lab } & \text { Unit } \\ <2.00 & 1 & & \text { GE } & \mu g / L \\ <5.00 & 1 & & & \text { WA } & \mu g / L \\ <2.00 & 1 & & & \text { GE } & \mu g / L \\ <5.00 & 1 & & & \text { WA } & \mu g / \\ 3.54 & 1 & & \text { GE } & \mu g / L \\ 2.7 & 1 & \text { JE } & & \text { WA } & \mu g / L \\ <5.00 & 1 & & & \text { WA } & \mu g / L\end{array}$

Note: Modifiers are listed and detailed in Appendix D, Key to Reading the Tables.

- = exceeded holding time. 
WELL LFW 31

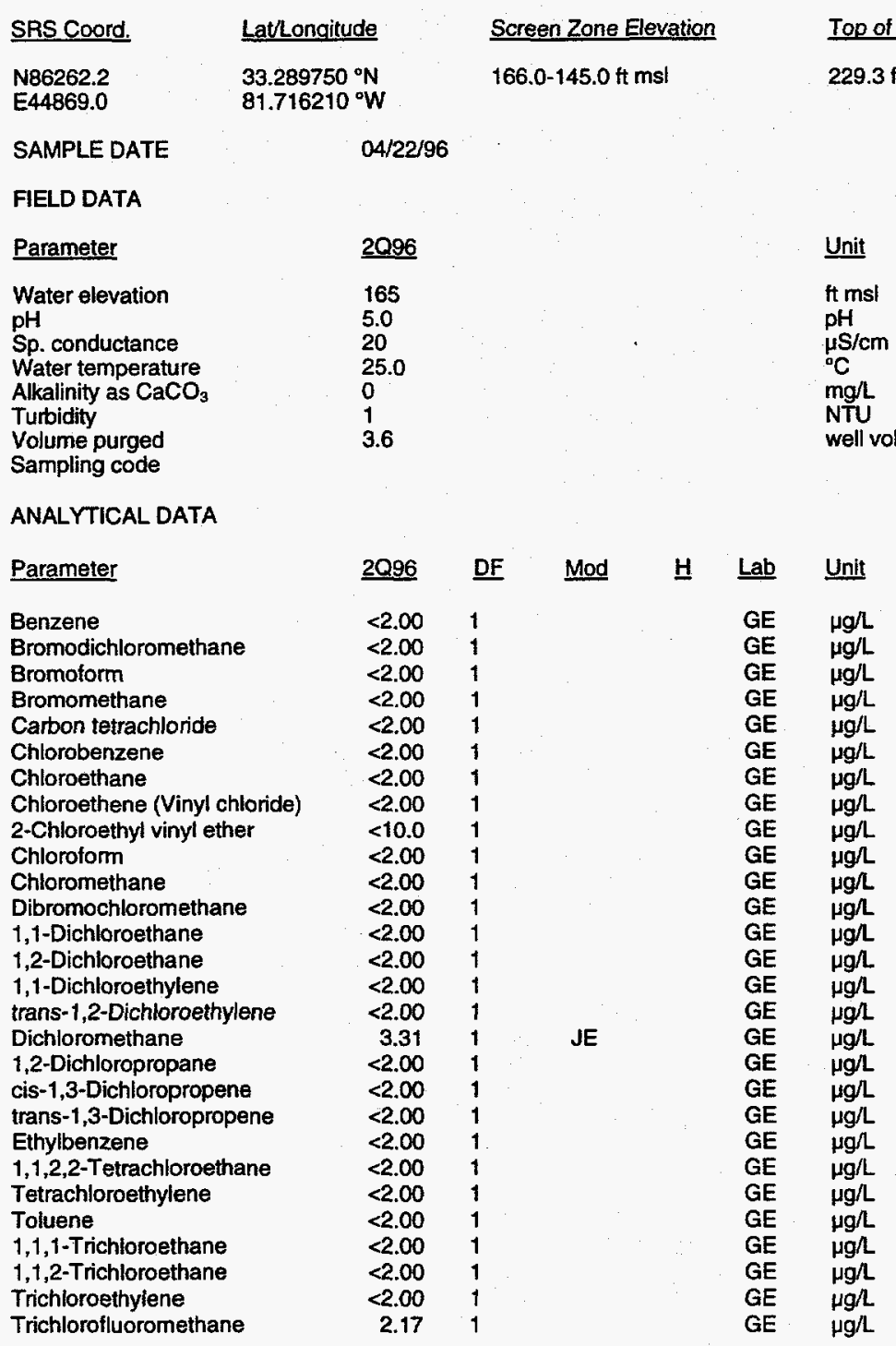

Note: Modifiers are listed and detailed in Appendix D, Key to Reading the Tables. $\bullet=$ exceeded holding time. 
WELLL LFW 32

SAS Coord.
N85836.8
E44935.9
SAMPLE DATE
FIELD DATA
Parameter
Water elevation
pH
Sp. conductance
Water temperature
Alkalinity as CaCO
Turbidity
Volume purged
Sampling code

Lathongitude

$33.288920^{\circ} \mathrm{N}$ $81.715210^{\circ} \mathrm{W}$

$04 / 23 / 96$

ANALYTICAL DATA

\section{Parameter}

Acenaphthylene

Acetone

Acetonitrile (Methyl cyanide)

Acetophenone

2-Acetylaminofluorene

Acrolein

Acrylonitrile

Aldrin

Allyl chloride

4-Aminobiphenyl

Aniline

Anthracene

Antimony, total recoverable

Aramite

Arsenic, total recoverable

Barium, total recoverable

Benzene

alpha-Benzene hexachloride

beta-Benzene hexachloride

delta-Benzene hexachloride

Benzo(a)anthracene

Benzo(b)fluoranthene

Benzo(k)fluoranthene

Benzo(g,h,i)perylene

Benzo(a)pyrene

Benzyl alcohol

Beryllium, total recoverable

Bis(2-chloroethoxy) methane

Bis(2-chloroethyl) ether

Bis(2-chloroisopropyl) ether

Bis(2-ethylhexyl) phthalate

Bromodichloromethane

Bromoform

Bromomethane

4-Bromophenyl phenyl ether

Butyibenzyl phthalate

2-sec-Butyl-4,6-dinitrophenol

Cadmium, total recoverable

Carbon disulfide

Carbon tetrachloride

Chlordane

4-Chloroaniline

Chlorobenzene

Chlorobenzilate

Chloroethane

Chloroethene (Vinyl chloride)

Chloroform

Chloromethane

2-Chloronaphthalene

4-Chiorophenyl phenyl ether
Screen Zone Elevation

165.3-144.3 ft ms
Top of Casing

$223.7 \mathrm{ft} \mathrm{ms}$
Casing

4" PVC
Pump

$\mathrm{S}$
Screen Zone

Upper Steed Pond
Unit

It msi

$\mathrm{pH}$

is/cm

$\mathrm{C}$

mg/l

well vol

\begin{tabular}{|c|c|c|c|}
\hline 2096 & DF & Mod & Lab \\
\hline$<1.00$ & 1 & & GE \\
\hline$<10.0$ & 1 & & GE \\
\hline$<10.0$ & 1 & & GE \\
\hline$<1.00$ & 1 & & GE \\
\hline$<10.0$ & 1 & & $\mathrm{GE}$ \\
\hline$<20.0$ & 1 & & GE \\
\hline$<50.0$ & 1 & & GE \\
\hline$<0.0123$ & 1 & & GE \\
\hline$<10.0$ & 1 & & GE \\
\hline$<10.0$ & 1 & & GE \\
\hline$<1.00$ & 1 & & GE \\
\hline$<1.00$ & 1 & & GE \\
\hline$<5.00$ & 1 & & GE \\
\hline$<10.0$ & 1 & & $\overline{G E}$ \\
\hline$<5.00$ & 1 & & GE \\
\hline 7.6 & 1 & V & GE \\
\hline$<2.00$ & 1 & & GE \\
\hline$<0.0123$ & 1 & & GE \\
\hline$<0.0245$ & 1 & & GE \\
\hline$<0.0245$ & 1 & & $\overline{\mathrm{GE}}$ \\
\hline$<0.100$ & 1 & & GE \\
\hline$<0.100$ & 1 & & GE \\
\hline$<0.100$ & 1 & & GE \\
\hline$<1.00$ & 1 & & GE \\
\hline$<0.100$ & 1 & & GE \\
\hline$<1.00$ & 1 & & GE \\
\hline$<0.0497$ & 1 & V & GE \\
\hline$<1.00$ & 1 & & GE \\
\hline$<1.00$ & 1 & & GE \\
\hline$<20.0$ & 1 & & $\overline{\mathrm{GE}}$ \\
\hline$<1.00$ & 1 & & GE \\
\hline$<2.00$ & 1 & & GE \\
\hline$<2.00$ & 1 & & GE \\
\hline$<2.00$ & 1 & & GE \\
\hline$<1.00$ & 1 & & GE \\
\hline$<1.00$ & 1 & & GE \\
\hline$<10.0$ & 1 & & GE \\
\hline$<2.00$ & $i$ & & 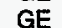 \\
\hline$<5.00$ & 1 & & GE \\
\hline$<2.00$ & 1 & & GE \\
\hline$<0.123$ & 1 & & GE \\
\hline$<1.00$ & 1 & & GE \\
\hline$<2.00$ & 1 & & GE \\
\hline$<10.0$ & 1 & & GE \\
\hline$<2.00$ & 1 & & GE \\
\hline$<2.00$ & 1 & & GE \\
\hline$<2.00$ & 1 & & GE \\
\hline$<2.00$ & 1 & & $\mathrm{GE}$ \\
\hline$<1.00$ & 1 & & GE \\
\hline$<1.00$ & 1 & & GE \\
\hline
\end{tabular}

Note: Modifiers are listed and detailed in Appendix D, Key to Reading the Tables.

- = exceeded holding time. 
Well LFW 32 (cont.)

\section{Parameter}

Chioroprene

Chromium, total recoverable

Chrysene

Cobalt, total recoverable

Copper, total recoverable

$\mathrm{m} / \mathrm{p}$-Cresol

o-Cresol (2-Methylphenol)

Cyanide

$p, p^{\prime}-D D D$

$p, p^{\prime}-D D E$

p,p'-DDT

Diallate

Dibenz(a,h)anthracene

Dibenzofuran

Dibromochloromethane

1,2-Dibromo-3-chloropropane

1,2-Dibromoethane

Dibromomethane

Di-n-butyl phthalate

1,2-Dichlorobenzene

1,3-Dichlorobenzene

3,3'-Dichlorobenzidine

trans-1,4-Dichloro-2-butene

Dichlorodifluoromethane

1,1-Dichloroethane

1,2-Dichloroethane

1,1-Dichloroethylene

trans-1,2-Dichloroethylene

Dichloromethane

24-Dichlorophenol

2,6-Dichlorophenal

2,4-Dichlorophenoxyacetic acid

1,2-Dichloropropane

cis-1,3-Dichloropropene

trans-1,3-Dichloropropene

Dieldrin

Diethyl phthalate

Dimethoate

2,4-Dimethyl phenol

Dimethyl phthalate

p-Dimethylaminoazobenzene

7,12-Dimethylbenz(a)anthracene

3,3'-Dimethylbenzidine

a,a-Dimethyiphenethylamine

1,3-Dinitrobenzene

2,4-Dinitrophenol

2,6-Dinitrotoluene

Di-n-octyl phthalate

1,4-Dioxane

Diphenylamine

Disulfoton

Endosulfan sulfate

Endosulfan I

Endosultan I

Endrin

Endrin aldehyde

Ethyl methacrylate

Ethyl methanesulfonate

Ethylbenzene

Famphur

Fluoranthene

Fluorene

Heptachlor

Heptachlor epoxide

Heptachlorodibenzo-p-dioxins

Heptachlorodibenzo-p-furans

Hexachlorobenzene

Hexachlorobutadiene

Hexachlorocyclopentadiene

Hexachlorodibenzo-p-dioxins

Hexachlorodibenzo-p-furans

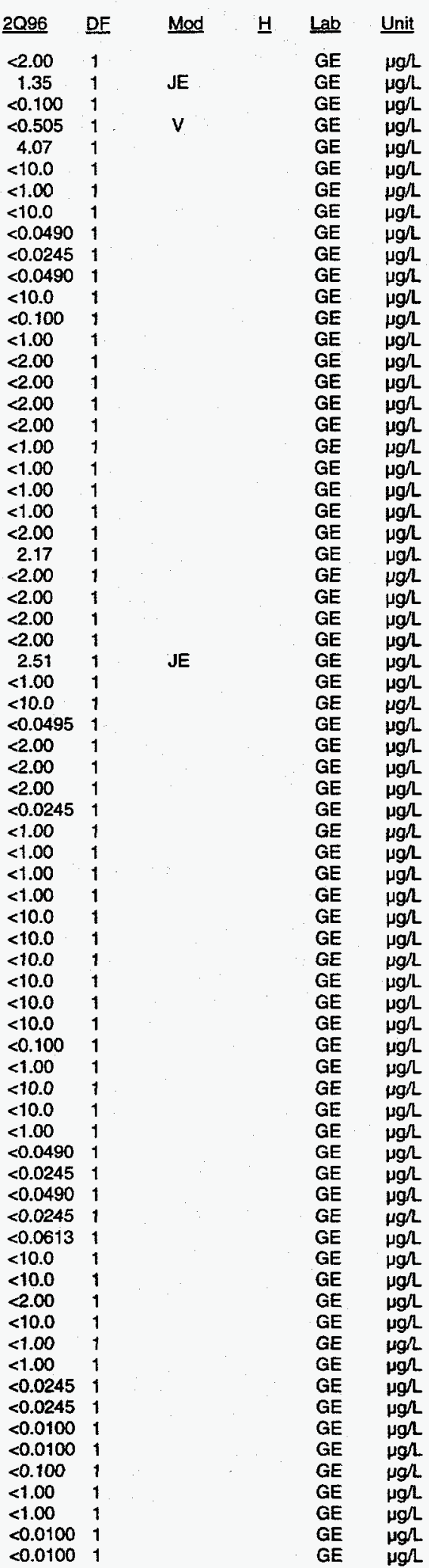

Note: Modifiers are listed and detailed in Appendix D, Key to Reading the Tables.

$\bullet=$ exceeded holding time. 
Well LFW 32 (cont.)

\begin{tabular}{|c|c|c|c|c|c|c|}
\hline Parameter & $2 Q 96$ & $\underline{\text { DF }}$ & Mod & $\underline{\mathbf{H}}$ & $\underline{L a b}$ & Unit \\
\hline Hexachloroethane & $<0.100$ & 1 & & & GE & $\mu g / L$ \\
\hline Hexachlorophene & $<80.0$ & 1 & & & GE & kgh \\
\hline Hexachloropropene & $<10.0$ & 1 & & & GE & jghl \\
\hline 2-Hexanone & $<10.0$ & 1 & & & $\overrightarrow{G E}$ & $\mathrm{pg} / \mathrm{h}$ \\
\hline $1,2,3,4,6,7,8-H P C D D$ & $<0.0100$ & 1 & & & GE & $\mu g / 2$ \\
\hline $1,2,3,4,6,7,8-H P C D F$ & $<0.0100$ & 1 & & & GE & $\mu g /$ \\
\hline $1,2,3,4,7,8-\mathrm{HXCDD}$ & $<0.0100$ & 1 & & & GE & $\mu g / L$ \\
\hline $1,2,3,4,7,8-H \times C D F$ & $<0.0100$ & 1 & & & GE & $\mu g / 2$ \\
\hline Indeno( $1,2,3-c, d)$ pyrene & $<0.100$ & 1 & & & GE & $\mu g /$ \\
\hline lodomethane (Methyl iodide) & $<10.0$ & 1 & & & GE & $\mu g / L$ \\
\hline Isobutyl alcohol & $<20.0$ & 1 & & & GE & $\mu g /$ \\
\hline Isodrin & $<10.0$ & 1 & & & GE & $\mu g /$ \\
\hline Isophorone & $<1.00$ & 1 & & & GE & $\mu g / L$ \\
\hline Isosafrole & $<10.0$ & 1 & & & GE & $\mu g / h$ \\
\hline Kepone & $<10.0$ & 1 & . & & GE & $\mu g /$ \\
\hline Lead, total recoverable & $<5.00$ & 1 & & & GE & $\mu g /$ \\
\hline Lindane & $<0.0123$ & 1 & & & GE & $g /$ \\
\hline Mercury, total recoverable & 0.047 & 1 & JE & & $\overrightarrow{G E}$ & $\mu g / L$ \\
\hline Methacrylonitrile & $<10.0$ & 1 & & & $\mathrm{GE}$ & $\mu g /$ \\
\hline Methapyrilene & $<10.0$ & 1 & & & GE & $\mu g /$ \\
\hline Methoxychlor & $<0.245$ & 1 & & & GE & $\mu g / 2$ \\
\hline 2-Methyl-4,6-dinitrophenol & $<10.0$ & 1 & & & GE & $\mu g / L$ \\
\hline Methyl ethyl ketone & $<5.00$ & 1 & & & GE & $\mu g /$ \\
\hline Methyl isobutyl ketone & $<10.0$ & 1 & & & GE & $\mu g / L$ \\
\hline Methyl methacrylate & $<10.0$ & 1 & & & GE & $\mu g / L$ \\
\hline Methyl methanesulfonate & $<10.0$ & 1 & & & GE & $\mu g / L$ \\
\hline 3-Methylcholanthrene & $<10.0$ & 1 & & & GE & $\mu g / L$ \\
\hline 2-Methyinaphthalene & $<1.00$ & 1 & & & GE & $\mu g / L$ \\
\hline Naphthalene & $<1.00$ & 1 & & & GE & $\mu g / L$ \\
\hline 1,4-Naphthoquinone & $<10.0$ & 1 & & & GE & 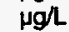 \\
\hline 1-Naphthylamine & $<10.0$ & 1 & & & GE & Hgl \\
\hline 2-Naphthylamine & $<10.0$ & 1 & & & GE & $\mu g /$ \\
\hline Nickel, total recoverable & $<10.0$ & 1 & & & GE & Hgh \\
\hline m-Nitroaniline & $<1.00$ & 1 & & & GE & $\mu g / L$ \\
\hline o-Nitroaniline & $<1.00$ & 1 & & & GE & $\mu g /$ \\
\hline p-Nitroaniline & $<1.00$ & 1 & & & GE & $\mu g /$ \\
\hline Nitrobenzene & $<1.00$ & 1 & & & GE & $\mu g /$ \\
\hline 2-Nitrophenal & $<1.00$ & 1 & & & GE & $\mu g / 2$ \\
\hline 4-Nitrophenol & $<1.00$ & 1 & 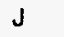 & & GE & jg/ \\
\hline 4-Nitroquinoline-1-oxide & $<10.0$ & 1 & & & GE & $\mu g /$ \\
\hline N-Nitrosodi-n-butylamine & $<10.0$ & 1 & & & GE & $\mu g /$ \\
\hline N-Nitrosodiethylamine & $<10.0$ & 1 & & & GE & $\mu g / L$ \\
\hline N-Nitrosodimethylamine & $<1.00$ & 1 & & & GE & $\mu \mathrm{g} / \mathrm{L}$ \\
\hline N-Nitrosodiphenylamine & $<1.00$ & 1 & & & GE & $\mu g / L$ \\
\hline N-Nitrosomethylethylamine & $<10.0$ & 1 & & & GE & $\mu g / L$. \\
\hline N-Nitrosomorpholine & $<10.0$ & 1 & & & GE & $\mu g /$ \\
\hline N-Nitrosopiperidine & $<10.0$ & 1 & & & GE & $\mu g / L$ \\
\hline N-Nitrosopymolidine & $<10.0$ & 1 & & & GE & $\mu g / 2$ \\
\hline 5-Nitro-o-toluidine & $<10.0$ & 1 & & & GE & $\mu g /$ \\
\hline Octachlorodibenzo-p-dioxin & $<0.0100$ & 1 & & & GE & $\mu g / L$ \\
\hline Octachlorodibenzo-p-furan & $<0.0100$ & 1 & & & GE & $\mu g /$. \\
\hline Parathion & $<0.0245$ & 1 & & & GE & $\mu g / L$ \\
\hline Parathion metthyl & $<0.0245$ & 1 & & & GE & $\mu g / L$ \\
\hline PCB 1016 & $<0.125$ & 1 & $\mathrm{~J}$ & - & GE & joh \\
\hline PCB 1016 & $<0.0613$ & 1 & & & GE & $\mu \mathrm{g} / \mathrm{L}$ \\
\hline РCB 1016 & $<0.125$ & $i$ & & & GE & $\mu g / L$ \\
\hline PCB 1221 & $<0.125$ & 1 & $\mathbf{J}$ & - & GE & $\mu g /$ \\
\hline PCB 1221 & $<0.0613$ & 1 & & & GE & $\mu g / L$ \\
\hline PCB 1221 & $<0.125$ & 1 & & & GE & $\mu g /$. \\
\hline PCB 1232 & $<0.125$ & 1 & $J$ & 0 & GE & $\mu g /$ \\
\hline PCB 1232 & $<0.0613$ & 1 & & & GE & $\mu g / L$ \\
\hline PCB 1232 & $<0.125$ & 1 & & & GE & $\mu g h$ \\
\hline PCB 1242 & $<0.125$ & 1 & $J$ & $\bullet$ & GE & jol \\
\hline PCB 1242 & $<0.0613$ & 1 & & & $\mathrm{GE}$ & $\mu g /$ \\
\hline PCB 1242 & $<0.125$ & 1 & & & GE & uol. \\
\hline PCB 1248 & $<0.125$ & 1 & $J$ & $\bullet$ & GE & $\mu g / L$ \\
\hline PCB 1248 & $<0.0613$ & 1 & & & & \\
\hline PCB 1248 & $<0.125$ & 1 & & & GE & $\mu \mathrm{g} / \mathrm{L}$ \\
\hline PCB 1254 & $<0.125$ & 1 & $\mathbf{J}$ & - & GE & $\mu g / L$ \\
\hline PCB 1254 & $<0.0613$ & 1 & & & $\mathrm{GE}$ & $\mu \mathrm{g} / \mathrm{L}$ \\
\hline PCB 1254 & $<0.125$ & 1 & & & GE & $\mu g / 2$ \\
\hline PCB 1260 & $<0.125$ & 1 & $\mathrm{~J}$ & & $\mathrm{GE}$ & $\mu \mathrm{g} /$ \\
\hline PCB 1260 & $<0.125$ & 1 & $J$ & - & GE & \\
\hline
\end{tabular}

Note: Modifiers are listed and detailed in Appendix D, Key to Reading the Tables.

- = exceeded holding time. 
Well LFW 32 (cont.)

\section{Parameter}

PCB 1260

$1,2,3,7,8-P C D D$

$1,2,3,7,8$-PCDF

Pentachlorobenzene

Pentachlorodibenzo-p-dioxins

Pentachlorodibenzo- $p$-furans

Pentachloroethane

Pentachloronitrobenzene

Phenacetin

Phenanthrene

p-Phenylenediamine

Phorate

2-Picoline

Pronamid

Propionitrile

Pyridine

Safrole

Selenium, total recoverable

Silver, total recoverable

Styrene

Sulfide

Sulfotepp

2,4,5-T

2,3,7,8-TCDD

2,3,7,8-TCDF

1,2,4,5-Tetrachlorobenzene

Tetrachlorodibenzo-p-dioxins

Tetrachlorodibenzo-p-furans

1,1,1,2-Tetrachloroethane

1,1,2,2-Tetrachloroethane

Tetrachloroethylene

2,3,4,6-Tetrachlorophenol

Thallium, total recoverable

Thionazin

Tin, total recoverable

Toluene

o-Toluidine

Toxaphene

2,4,5-TP (Silvex)

1,1,1-Trichloroethane

1,1,2-Trichloroethane

Trichloroethylene

Trichlorofluoromethane

2,4,5-Trichloropheno

2,4,6-Trichlorophenol

1,2,3-Trichloropropane

$0,0,0$-Triethyl phosphorothioate

1,3,5-Trinitrobenzene

Vanadium, total recoverable

Vinyl acetate

Xylenes

Zinc, total recoverable

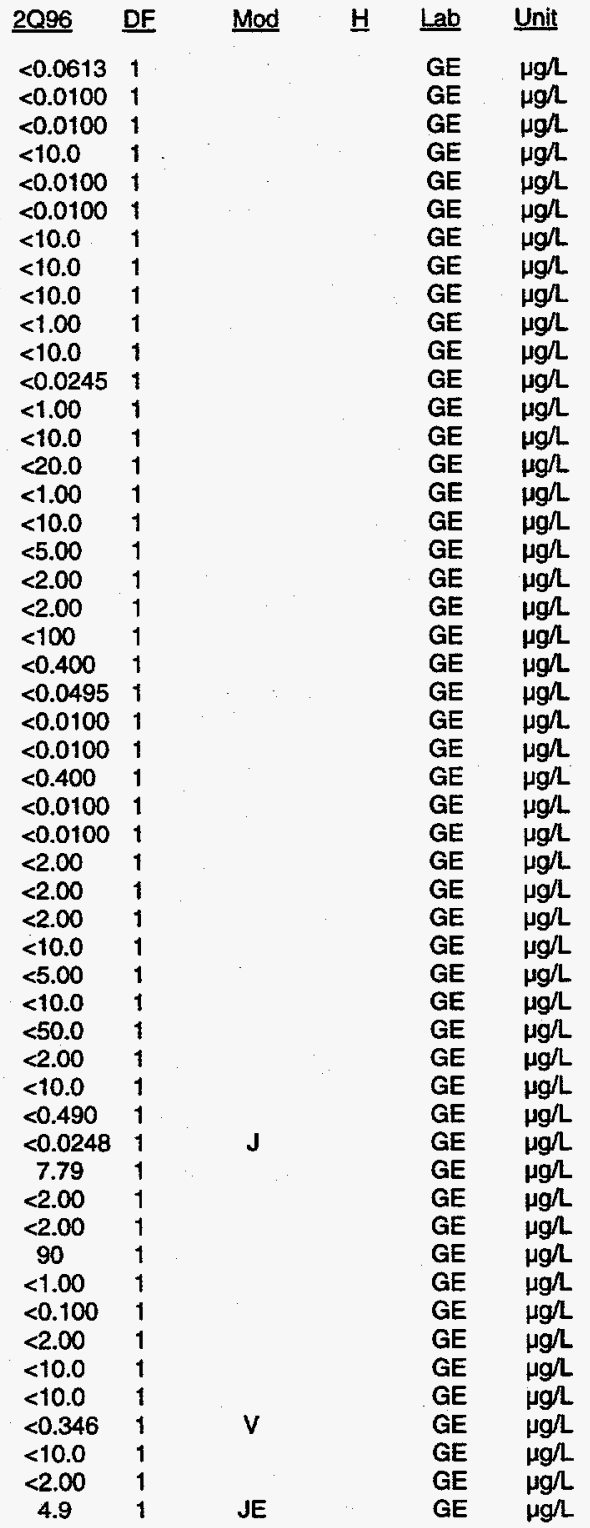

Note: Modifiers are listed and detailed in Appendix D, Key to Reading the Tables.

$\bullet=$ exceeded holding time. 
WELL LFW 33

\begin{tabular}{lc} 
SRS Coord. & Latlongitude \\
\cline { 2 - 2 } N85633.8 & $33.288530^{\circ} \mathrm{N}$ \\
E44973.0 & $81.714720^{\circ} \mathrm{W}$ \\
SAMPLE DATE & $04 / 22 / 96$ \\
FIELD DATA &
\end{tabular}

Screen Zone Elevation

$165.4-144.4 \mathrm{ft} \mathrm{ms}$

2096

161.7

4.6

24

22.0

0

3.1
Top of Casing

213.7 ft ms

Casing

4" PVC
Pump

$\mathrm{S}$
Screen Zone

Upper Steed Pond
Unit

it msl

$\mathrm{pH}$

pS/cm

${ }^{\circ} \mathrm{C}$

mg/t

well vol

Turbidity

Sampling code

ANALYTICAL DATA

Parameter

Benzene

Bromodichloromethane

Bromoform

Bromomethane

Carbon tetrachloride

Chlorobenzene

Chiloroethane

Chloroethene (Vinyl chloride)

2-Chioroethyl vinyl ether

Chlorotorm

Chloromethane

Dibromochloromethane

1,1-Dichloroethane

1,2-Dichloroethane

1,1-Dichloroethylene

trans-1,2-Dichloroethylene

Dichloromethane

1,2-Dichloropropane

cis-1,3-Dichloropropene

trans-1,3-Dichloropropene

Ethylbenzene

1,1,2,2-Tetrachioroethane

Tetrachloroethylene

Toluene

1,1,1-Trichloroethane

1,1,2-Trichloroethane

Trichloroethylene

Trichlorofluoromethane

$\begin{array}{ll}2 Q 96 & \text { D } \\ <2.00 & 1 \\ <2.00 & 1 \\ <2.00 & 1 \\ <2.00 & 1 \\ <2.00 & 1 \\ <2.00 & 1 \\ <2.00 & 1 \\ <2.00 & 1 \\ <10.0 & 1 \\ <2.00 & 1 \\ <2.00 & 1 \\ <2.00 & 1 \\ <2.00 & 1 \\ <2.00 & 1 \\ <2.00 & 1 \\ <2.00 & 1 \\ 3.04 & 1 \\ <2.00 & 1 \\ <2.00 & 1 \\ <2.00 & 1 \\ <2.00 & 1 \\ <2.00 & 1 \\ <2.00 & 1 \\ <2.00 & 1 \\ 9.99 & 1 \\ <2.00 & 1 \\ <2.00 & 1 \\ 121 & 1\end{array}$

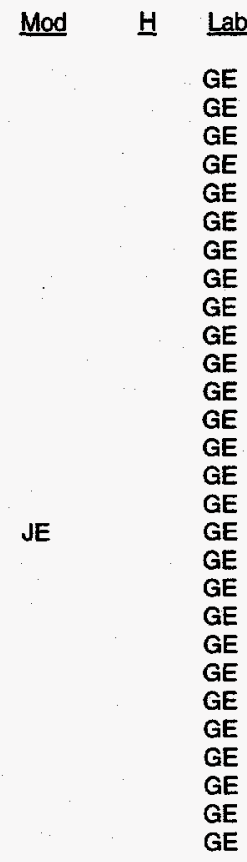

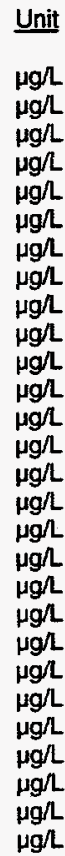

Note: Modifiers are listed and detailed in Appendix D, Key to Reading the Tables.

- exceeded holding time. 
WELLL LFW 34

SRS Coord.

N85409.5

E45016.9

SAMPLE DATE

FIELD DATA

Parameter

Water elevation

$\mathrm{pH}$

Sp. conductance

Water temperature

Alkalinity as $\mathrm{CaCO}$

Turbidity

Volume purged

Sampling code

ANALYTICAL DATA

Parameter

Benzene

Bromodichloromethane

Bromolorm

Bromomethane

Carbon tetrachloride

Chlorobenzene

Chloroethane

Chloroethene (Vinyl chloride)

2-Chloroethyl vinyl ether

Chloroform

Chloromethane

Dibromochloromethane

1,1-Dichloroethane

1,2-Dichloroethane

1,1-Dichloroethylene

trans-1,2-Dichloroethylene

Dichloromethane

1,2-Dichloropropane

cis-1,3-Dichloropropene

trans-1,3-Dichloropropene

Ethylbenzene

1,1,2,2-Tetrachloroethane

Tetrachioroethylene

Toluene

1,1,1-Trichloroethane

1,1,2-Trichloroethane

Trichloroethylene

Trichlorofluoromethane
Lathongitude

$33.288110^{\circ} \mathrm{N}$ $81.714170^{\circ} \mathrm{W}$

04/22/96

$\underline{2096}$

160.4

4.4

20

24.0

0

3.4

Screen Zone Elevation

$164.7-143.7 \mathrm{ft} \mathrm{msl}$

Top of Casing

Casing

Pump

$201 \mathrm{ft} \mathrm{rnst}$

4" PVC

$\mathrm{S}$

Unit

it ms

$\mathrm{pH}$

$\mu \mathrm{S} / \mathrm{cm}$

$\mathrm{mg}$

NTU

well vol
Screen Zone

Upper Steed Pond

\begin{tabular}{|c|c|c|}
\hline 2Q96 & DF & $\underline{\text { Mod }}$ \\
\hline$<2.00$ & 1 & \\
\hline$<2.00$ & 1 & \\
\hline$<2.00$ & 1 & \\
\hline$<2.00$ & 1 & \\
\hline$<2.00$ & 1 & \\
\hline$<2.00$ & 1 & \\
\hline$<2.00$ & 1 & \\
\hline$<2.00$ & 1 & \\
\hline$<10.0$ & 1 & \\
\hline$<2.00$ & 1 & \\
\hline$<2.00$ & 1 & \\
\hline$<2.00$ & 1 & \\
\hline$<2.00$ & 1 & \\
\hline$<2.00$ & 1 & \\
\hline$<2.00$ & 1 & \\
\hline$<2.00$ & 1 & \\
\hline 1.71 & 1 & JE \\
\hline$<2.00$ & 1 & \\
\hline$<2.00$ & 1 & \\
\hline$<2.00$ & 1 & \\
\hline$<2.00$ & 1 & \\
\hline$<2.00$ & 1 & \\
\hline$<2.00$ & 1 & \\
\hline$<2.00$ & 1 & \\
\hline 1.81 & 1 & JE \\
\hline$<2.00$ & 1 & \\
\hline$<2.00$ & 1 & \\
\hline 18.8 & 1 & \\
\hline
\end{tabular}

Note: Modifiers are listed and detailed in Appendix D, Key to Reading the Tables.

- = exceeded holding time. 
WELL LFW 43C

\begin{tabular}{|c|c|c|c|c|c|c|}
\hline SRS Coord. & LavLongitude & Screen Zone Elevation & Top of Casing & Casing & Pump & Screen Zone \\
\hline $\begin{array}{l}\text { N86480.6 } \\
\text { E45234.9 }\end{array}$ & $\begin{array}{l}33.290830^{\circ} \mathrm{N} \\
81.715670^{\circ} \mathrm{W}\end{array}$ & $138.5-128.5 \mathrm{ft} \mathrm{msl}$ & $202.6 \mathrm{ft} \mathrm{msl}$ & $4 * P V C$ & $s$ & Middle Steed Pond \\
\hline FIELD DATA & & & & & & \\
\hline Parameter & $\underline{2096}$ & & Unit & & & \\
\hline $\begin{array}{l}\text { Water elevation } \\
\text { pH } \\
\text { Sp. conductance } \\
\text { Water temperature } \\
\text { Alkalinity as } \mathrm{CaCO}_{3} \\
\text { Turbidity } \\
\text { Volume purged }\end{array}$ & $\begin{array}{l}165.9 \\
5 \\
14 \\
25.0 \\
1 \\
0 \\
5.4\end{array}$ & & $\begin{array}{l}\text { ft msl } \\
\mathrm{pH} \\
\mu \mathrm{S} / \mathrm{cm} \\
{ }^{\circ} \mathrm{C} \\
\mathrm{mg} / \mathrm{L} \\
\text { NTU } \\
\text { well vol }\end{array}$ & & & \\
\hline
\end{tabular}

ANALYTICAL DATA

\begin{tabular}{|c|c|c|c|c|c|}
\hline Parameter & $\underline{2 Q 96}$ & $\underline{\text { DF }}$ & Mod & $\underline{H}$ & Lab \\
\hline 1, f-Dichloropropene & $<5.00$ & 1 & & & WA \\
\hline Acenaphthene & $<1.00$ & 1 & & & GE \\
\hline Acenaphthene & $<10.0$ & 1 & & & WA \\
\hline Acenaphthylene & $<1.00$ & 1 & & & GE \\
\hline Acenaphthylene & $<1.00$ & 1 & & & GE \\
\hline Acenaphthylene & $<10.0$ & 1 & & & WA \\
\hline Acetone & $<10.0$ & 1 & & & GE \\
\hline Acetone & $<10.0$ & 1 & & & WA \\
\hline Acetonitrile (Methyl cyanide) & $<10.0$ & 1 & & & GE \\
\hline Acetonitrile (Methyl cyanide) & $<20.0$ & 1 & & & WA \\
\hline Acetophenone & $<1.00$ & 1 & & & GE \\
\hline Acetophenone & $<1.00$ & 1 & & & GE \\
\hline Acetophenone & $<10.0$ & 1 & & & WA \\
\hline 2-Acetylaminofluorene & $<10.0$ & 1 & & & GE \\
\hline 2-Acetylaminofluorene & $<10.0$ & 1 & & & GE \\
\hline 2-Acetylaminofluorene & $<10.0$ & 1 & & & WA \\
\hline Acrolein & $<20.0$ & 1 & & & GE \\
\hline Acrolein & $<10.0$ & 1 & & & WA \\
\hline Acrylonitrile & $<50.0$ & 1 & & & GE \\
\hline Acrylonitrile & $<10.0$ & 1 & & & WA \\
\hline Aldrin & $<0.0124$ & 1 & & & GE \\
\hline Aldrin & $<0.0500$ & 1 & & . & WA \\
\hline Allyl chloride & $<10.0$ & 1 & & & GE \\
\hline Aliyl chioride & $<20.0$ & 1 & & & WA \\
\hline 4-Áminobiphenyl & $<10.0$ & 1 & & & GE \\
\hline 4-Aminobiphenyl & $<10.0$ & 1 & & & GE \\
\hline 4-Aminobiphenyl & $<10.0$ & 1 & & & WA \\
\hline Aniline & $<1.00$ & 1 & & & GE \\
\hline Aniline & $<1.00$ & 1 & & & GE \\
\hline Aniline & $<10.0$ & 1 & & & WA \\
\hline Anthracene & $<1.00$ & 1 & & & GE \\
\hline Anthracene & $<1.00$ & 1 & & & GE \\
\hline Anthracene & $<10.0$ & 1 & & & WA \\
\hline Antimony, total recoverable & $<5.00$ & 1 & & & GE \\
\hline Antimony, total recoverable & $<27.0$ & 1 & & & WA \\
\hline Antimony, total recoverable & $<27.0$ & 1 & & & WA \\
\hline Aramite & $<10.0$ & 1 & & & GE \\
\hline Aramite & $<10.0$ & 1 & & & GE \\
\hline Aramite & $<20.0$ & 1 & & & WA \\
\hline Arsenic, total recoverable & $<5.00$ & 1 & & & GE \\
\hline Arsenic, total recoverable & $<40.0$ & 1 & & & WA \\
\hline Arsenic, total recoverable & $<40.0$ & 1 & & & WA \\
\hline Barium, total recoverable & 4.15 & 1 & $v$ & & GE \\
\hline Barium, total recoverable & 4.4 & $t$ & & & WA \\
\hline Barium, total recoverable & 4.1 & 1 & & & WA \\
\hline Benzene & $<2.00$ & 1 & & & GE \\
\hline Benzene & $<5.00$ & 1 & & & WA \\
\hline alpha-Benzene hexachloride & $<0.0124$ & 1 & & & GE \\
\hline alpha-Benzene hexachloride & $<0.0500$ & 1 & & & WA \\
\hline beta-Benzene hexachloride & $<0.0248$ & 1 & & & GE \\
\hline beta-Benzene hexachloride & $<0.0500$ & 1 & & & WA \\
\hline
\end{tabular}

Note: Modifiers are listed and detalied in Appendix D, Key to Reading the Tables. $\bullet=$ exceeded holding time. 
Well LFW 43C (cont.)

\begin{tabular}{|c|c|c|c|c|c|c|}
\hline Parameter & $\underline{2096}$ & DF & Mod & $\underline{H}$ & $\underline{L a b}$ & Unit \\
\hline delta-Benzene hexachloride & $<0.0248$ & 1 & & & GE & $\mu g / L$ \\
\hline delta-Benzene hexachloride & $<0.0500$ & 1 & & & WA & $\mu g h$ \\
\hline Benzo(a)anthracene & $<0.100$ & 1 & & & GE & $\mu g / L$ \\
\hline Benzo(a)anthracene & $<0.100$ & 1 & & & GE & $\mu g / L$ \\
\hline Benzo(a)anthracene & $<10.0$ & 1 & & & WA & $\mu g / L$ \\
\hline Benzo(b)fluoranthene & $<0.100$ & 1 & & & GE & $\mu g / 2$ \\
\hline Benzo(b)fluoranthene & $<0.100$ & $i$ & & & GE & $\mu g h$. \\
\hline Benzo(b)fluoranthene & $<10.0$ & 1 & & & WA & $\mu g h$ \\
\hline Benzo(k)fluoranthene & $<0.100$ & 1 & & & GE & $\mu g / L$ \\
\hline Benzo(k)fluoranthene & $<0.100$ & 1 & & & GE & $\mu g /$ L. \\
\hline Benzo(k)ftuoranthene & $<10.0$ & 1 & & & WA & $\mu g h$ \\
\hline Benzoic acid & $<50.0$ & 1 & & & WA & $\mu g / L$ \\
\hline Benzo(g,h,i)perylene & $<1.00$ & 1 & & & GE & $\mu g / L$ \\
\hline Benzo(g,h,i)perylene & $<1.00$ & 1 & & & GE & $\mu g / L$ \\
\hline Benzo(g,h,i)perylene & $<10.0$ & 1 & & & WA & \\
\hline Benzo(a)pyrene & $<0.100$ & 1 & & & GE & $\mu g / L$ \\
\hline Benzo(a)pyrene & $<0.100$ & 1 & & & GE & $\mu g / L$ \\
\hline Benzo(a)pyrene & $<10.0$ & 1 & & & WA & $\mu g / L$. \\
\hline Benzyl alcohol & $<1.00$ & $t$ & & & GE & $\mu g / L$ \\
\hline Benzyl alcohol & $<1.00$ & 1 & & & GE & $\mu g / L$ \\
\hline Benzyl alcohol & $<10.0$ & 1 & & & WA & $\mu g / L$ \\
\hline Beryllium, total recoverable & $<0.0951$ & 1 & $\mathrm{v}$ & & GE & $\mu g h$ \\
\hline Beryllium, total recoverable & $<0.680$ & 1 & $v$ & & WA & $\mu g / L$ \\
\hline Beryllium, total recoverable & $<0.840$ & 1 & $\mathrm{v}$ & & WA & $\mu g / L$ \\
\hline Bis (2-chloroethoxy) methane & $<1.00$ & 1 & & & GE & $\mu g / L$ \\
\hline Bis(2-chloroethoxy) methane & $<1.00$ & 1 & & & GE & $\mu g / L$ \\
\hline Bis(2-chloroethoxy) methane & $<10.0$ & 1 & & & WA & $\mu g / L$ \\
\hline Bis(2-chloroethyl) ether & $<1.00$ & 1 & & & GE & $\mu g / L$ \\
\hline Bis(2-chloroethyl) ether & $<1.00$ & 1 & & & GE & $\mu g / L$ \\
\hline Bis(2-chloroethyl) ether & $<10.0$ & 1 & & & WA & $\mu g / L$ \\
\hline Bis(2-chloroisopropyl) ether & $<20.0$ & 1 & & & GE & $\mu g /$. \\
\hline Bis(2-chloroisopropyl) ether & $<10.0$ & 1 & & & WA & $\mu g / L$ \\
\hline Bis(2-ethylhexyl) phthalate & $<1.00$ & 1 & & & GE & $\mu g h$ \\
\hline Bis(2-ethylhexyl) phthalate & $<1.00$ & 1 & & & GE & $\mu g / 2$ \\
\hline Bis(2-ethylhexyl) phthalate & $<3.14$ & 1 & v & & WA & $\mu g / 2$ \\
\hline Bromobenzene & $<5.00$ & 1 & & & WA & $\mu g h$ \\
\hline Bromochloromethane & $<5.00$ & 1 & & & WA & $\mu g / h$ \\
\hline Bromodichloromethane & $<2.00$ & 1 & & & GE & $\mu g / L$ \\
\hline Bromodichloromethane & $<5.00$ & 1 & & & WA & $\mu g / L$ \\
\hline Bromoform & $<2.00$ & 1 & & & GE & $\mu g / L$ \\
\hline Bromoform & $<5.00$ & 1 & & & WA & ugl \\
\hline Bromomethane & $<2.00$ & 1 & & & GE & $\mu g / L$ \\
\hline Bromomethane & $<10.0$ & 1 & & & WA & $\mu g / L$ \\
\hline 4-Bromophenyl phenyl ether & $<1.00$ & 1 & & & GE & $\mu g / L$ \\
\hline 4-Bromophenyl phenyl ether & $<1.00$ & 1 & & & $\mathrm{GE}$ & $\mu g / L$ \\
\hline 4-Bromophenyl phenyl ether & $<10.0$ & 1 & & & WA & $\mu g / L$ \\
\hline n-Butylbenzene & $<5.00$ & 1 & & & WA & $\mu g / L$ \\
\hline sec-Butylbenzene & $<5.00$ & 1 & & & WA & $\mu g / L$ \\
\hline tert-Butybenzene & $<5.00$ & 1 & & & WA & $\mu g / L$ \\
\hline Butylbenzyl phthalate & $<1.00$ & 1 & & & GE & $\mu g / L$ \\
\hline Butylbenzyl phthalate & $<1.00$ & 1 & & & GE & $\mu g /$ \\
\hline Butylbenzyl phthalate & $<10.0$ & 1 & & & WA & $\mu g / L$ \\
\hline 2-sec-Butyl-4,6-dinitrophenol & $<10.0$ & 1 & & & $\mathrm{GE}$ & $\mu g / L$ \\
\hline 2-sec-Butyl-4,6-dinitrophenol & $<10.0$ & 1 & & & $\mathrm{GE}$ & $\mu g h$ \\
\hline 2-sec-Butyl-4,6-dinitrophenol & $<50.0$ & 1 & & & WA & $\mu g h$ \\
\hline Cadmium, total recoverable & $<2.00$ & 1 & & & GE & $\mu g / L$ \\
\hline Cadmium, total recoverable & 0.57 & 1 & JE & & WA & $\mu g / L$ \\
\hline Cadmium, total recoverable & $<4.70$ & 1 & $J$ & & WA & $\mu g / L$ \\
\hline Carbon disulfide & $<5.00$ & 1 & & & GE & $\mu g h$. \\
\hline Carbon disulfide & $<5.00$ & 1 & & & WA & $\mu g / L$ \\
\hline Carbon tetrachloride & $<2.00$ & 1 & & & GE & $\mu g / L$. \\
\hline Carbon tetrachloride & $<5.00$ & 1 & & & WA & igh \\
\hline Chlordane & $<0.124$ & 1 & & & GE & $\mu g / L$ \\
\hline alpha-Chiordane & $<0.0500$ & 1 & & & WA & $\mu g h$ \\
\hline gamma-Chlordane & $<0.0500$ & 1 & & & WA & \\
\hline 4-Chloroaniline & $<1.00$ & 1 & & & $\mathrm{GE}$ & $\mu g h$ \\
\hline 4-Chloroaniline & $<1.00$ & 1 & & & GE & \\
\hline 4-Chloroaniline & $<10.0$ & 1 & & & WA & $\mu g \Omega$ \\
\hline Chlorobenzene & $<2.00$ & 1 & & & $\mathrm{GE}$ & $\mu g h$ \\
\hline Chlorobenzene & $<5.00$ & 1 & & & WA & $\mu g \Omega$ \\
\hline Chlorobenzilate & $<10.0$ & 1 & & & GE & $\mu g h$ \\
\hline Chlorobenzilate & $<10.0$ & 1 & & & GE & \\
\hline
\end{tabular}

Note: Modifiers are listed and detailed in Appendix D, Key to Reading the Tabies. $\bullet$ = exceeded holding time. 
Well LFW 43C (cont.)

Parameter

\begin{tabular}{|c|c|c|c|c|}
\hline$\underline{2096}$ & DF & Mod & $\underline{H}$ & $\underline{\text { Lab }}$ \\
\hline$<10.0$ & 1 & & & WA \\
\hline$<1.00$ & 1 & & & $\mathrm{GE}$ \\
\hline$<10.0$ & 1 & & & WA \\
\hline$<2.00$ & 1. & & & GE \\
\hline$<10.0$ & 1 & & & WA \\
\hline$<2.00$ & 1 & & & GE \\
\hline$<10.0$ & 1 & & & WA \\
\hline$<10.0$ & 1 & & & WA \\
\hline$<2.00$ & 1 & & & GE \\
\hline$<5.00$ & 1 & & & WA \\
\hline$<2.00$ & 1 & & & GE \\
\hline$<10.0$ & 1 & & & WA \\
\hline$<1.00$ & 1 & & & $\mathrm{GE}$ \\
\hline$<1.00$ & 1 & & & $\mathrm{GE}$ \\
\hline$<10.0$ & 1 & & & WA \\
\hline$<1.00$ & 1 & $J$ & & $\mathrm{GE}$ \\
\hline$<10.0$ & 1 & & & WA \\
\hline$<1.00$ & 1 & & & $\mathrm{GE}$ \\
\hline$<1.00$ & 1 & & & GE \\
\hline$<10.0$ & 1 & & & WA \\
\hline$<2.00$ & 1 & & & GE \\
\hline$<5.00$ & 1 & & & WA \\
\hline$<5.00$ & 1 & & & WA \\
\hline$<5.00$ & 1 & & & WA \\
\hline$<4.00$ & 1 & & & $\mathrm{GE}$ \\
\hline$<7.00$ & 1 & & & WA \\
\hline$<7.00$ & 1 & & & WA \\
\hline$<0.100$ & 1 & & & $\mathrm{GE}$ \\
\hline$<0.100$ & 1 & & & $\overline{G E}$ \\
\hline$<10.0$ & 1 & & & WA \\
\hline$<0.362$ & 1 & v & & GE \\
\hline$<4.50$ & 1 & & & WA \\
\hline$<4.50$ & 1 & & & WA \\
\hline 1.65 & 1 & JE & & GE \\
\hline$<15.0$ & 1 & & & WA \\
\hline$<15.0$ & 1 & & & WA \\
\hline$<10.0$ & 1 & & & $\mathrm{GE}$ \\
\hline$<10.0$ & 1 & & & GE \\
\hline$<1.00$ & 1 & & & $\mathrm{GE}$ \\
\hline$<1.00$ & 1 & & & $\overline{G E}$ \\
\hline$<10.0$ & $i$ & & & WA \\
\hline$<10.0$ & 1 & & & WA \\
\hline$<10.0$ & 1 & & & $\mathrm{GE}$ \\
\hline$<1.95$ & 1 & V & & WA \\
\hline$<0.0495$ & $i$ & & & $\mathrm{GE}$ \\
\hline$<0.100$ & 1 & & & WA \\
\hline$<0.0248$ & 1 & & & GE \\
\hline$<0.100$ & 1 & & & WA \\
\hline$<0.0495$ & 1 & & & GE \\
\hline$<0.100$ & 1. & . & & WA \\
\hline$<10.0$ & 1 & & & $\mathrm{GE}$ \\
\hline$<10.0$ & 1 & & & GE \\
\hline$<10.0$ & $i$ & & & WA \\
\hline$<0.100$ & 1 & & & $\mathrm{GE}$ \\
\hline$<0.100$ & $i$ & & & $\mathrm{GE}$ \\
\hline$<10.0$ & 1 & & & WA \\
\hline$<1.00$ & 1 & & & $\mathrm{GE}$ \\
\hline$<1.00$ & 1 & & & GE \\
\hline$<10.0$ & $i$ & & & WA \\
\hline$<2.00$ & 1 & & & $\mathrm{GE}$ \\
\hline$<5.00$ & 1 & & & WA \\
\hline$<2.00$ & 1 & & & $\mathrm{GE}$ \\
\hline$<10.0$ & $i$ & & & WA \\
\hline$<2.00$ & 1 & & & GE \\
\hline$<10.0$ & 1 & & & WA \\
\hline$<2.00$ & 1 & & & $\mathrm{GE}$ \\
\hline$<10.0$ & 1 & & & WA \\
\hline$<1.00$ & 1 & & & $\mathrm{GE}$ \\
\hline$<1.00$ & 1 & & & $\mathrm{GE}$ \\
\hline$<10.0$ & 1 & & & WA \\
\hline$<1.00$ & $i$ & & & $\mathrm{GE}$ \\
\hline$<1.00$ & 1 & & & $\mathrm{GE}$ \\
\hline$<5.00$ & 1 & & & WA \\
\hline
\end{tabular}

Chlorobenzilate

4-Chloro-m-cresol

4-Chloro-m-cresol

Chloroethane

Chloroethane

Chloroethene (Vinyl chloride)

Chloroethene (Vinyl chloride)

2-Chloroethyl vinyl ether

Chloroform

Chloroform

Chloromethane

Chloromethane

2-Chloronaphthalene

2-Chloronaphthalene

2-Chioronaphthalene

2-Chlorophenol

2-Chlorophenol

4-Chlorophenyl phenyl ether

4-Chlorophenyl phenyl ether

4-Chlorophenyl phenyl ether

Chloroprene

Chloroprene

2-Chlorotoluene

4-Chlorotoluene

Chromium, total recoverable

Chromium, total recoverable

Chromium, total recoverable

Chrysene

Chrysene

Chrysene

Cobalt, total recoverable

Cobalt, total recoverable

Cobalt, total recoverable

Copper, total recoverable

Copper, total recoverable

Copper, total recoverable

mip-Cresol

mip-Cresol

o-Cresol (2-Methylphenol)

o-Cresol (2-Methylphenol)

o-Cresol (2-Methylphenol)

p-Cresol (4-Methylphenol)

Cyanide

Cyanide

$p, p^{\prime}-D D D$

$p, p^{\prime}-D D D$

P.P'-DDE

p,p'-DDE

$p, p^{\prime}-D D T$

p,p'-DDT

Diallate

Diallate

Diallate

Dibenz(a,h)anthracene

Dibenz(a,h)anthracene

Dibenz(a,h)anthracene

Dibenzofuran

Dibenzofuran

Dibenzofuran

Dibromochloromethane

Dibromochloromethane

1,2-Dibromo-3-chloropropane

1,2-Dibromo-3-chloropropane

1,2-Dibromoethane

1,2-Dibromoethane

Dibromomethane

Dibromomethane

Di-n-butyl phthalate

Di-n-butyl phthalate

Di-n-butyl phthalate

1,2-Dichlorobenzene

1,2-Dichlorobenzene

$<5.00$

Note: Modifiers are listed and detailed in Appendix D, Key to Reading the Tables.

- = exceeded holding time. 
Well LFW 43C (cont.) Parameter

1,2-Dichlorobenzene 1,3-Dichlorobenzene 1,3-Dichlorobenzene 1,3-Dichlorobenzene 1,3-Dichlorobenzene 1,4-Dichlorobenzene 1,4-Dichlorobenzene 1,4-Dichlorobenzene 3,3'-Dichlorobenzidine 3,3-Dichlorobenzidine 3,3-Dichlorobenzidine trans-1,4-Dichloro-2-butene trans-1,4-Dichloro-2-butene Dichlorodifluoromethane Dichlorodifluoromethane

1,1-Dichloroethane

1,1-Dichloroethane

1,2-Dichloroethane

1,2-Dichloroethane

1,1-Dichloroethylene

1,1-Dichloroethylene cis-1,2-Dichloroethylene

trans-1,2-Dichloroethylene trans-1,2-Dichloroethylene Dichloromethane

Dichloromethane

2,4-Dichlorophenol

2,4-Dichlorophenol

2,4-Dichloropheno

2.6-Dichlorophenol

2,6-Dichlorophenol

2,6-Dichlorophenol

2,4-Dichlorophenoxyacetic acid

2,4-Dichlorophenoxyacetic acid

2,4-Dichlorophenoxyacetic acid

2,4-Dichlorophenoxyacetic acid

1,2-Dichloropropane

1,2-Dichloropropane

1,3-Dichloropropane

2,2-Dichloropropane

cis-1,3-Dichioropropene

cis-1,3-Dichloropropene

trans-1,3-Dichloropropene

trans-1,3-Dichloropropene

Dieldrin

Dieldrin

Diethyl ether

Diethyl phthalate

Diethyl phthalate

Diethyl phthalate

Dimethoate

Dimethoate

Dimethoate

2,4-Dimethyl phenol

2,4-Dimethyl phenol

2,4-Dimethyl phenol

Dimethyl phthalate

Dimethyl phthalate

Dimethyl phthaiate

p-Dimethylaminoazobenzene

p-Dimethylaminoazobenzene

p-Dimethylaminoazobenzene

7,12-Dimethylbenz(a)anthracene

7,12-Dimethylbenz(a)anthracene

7,12-Dimethylbenz(a)anthracene

3,3'-Dimethylbenzidine

3,3'-Dimethylbenzidine

3,3'-Dimethylbenzidine

a,a-Dimethylphenethylamine

a,a-Dimethylphenethylamine

a,a-Dimethyiphenethylamine

1,3-Dinitrobenzene

1,3-Dinitrobenzene
2096

$<10.0$

$<1.00$

$<1.00$

$<5.00$

$<10.0$

$<1.00$

$<5.00$

$<10.0$

$<1.00$

$<1.00$

$<20.0$

$<2.00$

$<20.0$

$<2.00$

$<10.0$

$<200$

$<5.00$

$<2.00$

$<5.00$

$<2.00$

$<5.00$

$<5.00$

$<2.00$

$<5.00$

2.37

$<4.86$

$<1.00$

$<1.00$

$<10.0$

$<10.0$

$<10.0$

$<10.0$

$<0.0490$

$<0.0495$

$<1.02$

$<1.01$

$<5.00$

$<5.00$

$<5.00$

$<2.00$

$<5.00$

$<2.00$

$<5.00$

$<0.0248$

$<0.100$

$<10.0$

$<1.00$

$<1.00$

$<10.0$

$<1.00$

$<1.00$

$<0.206$

$<1.00$

$<1.00$

$<10.0$

$<1.00$

$<1.00$

$<10.0$

$<10.0$

$<10.0$

$<10.0$

$<10.0$

$<10.0$

$<10.0$

$<10.0$

$<10.0$

$<10.0$

$<10.0$

$<10.0$

$<10.0$

$<10.0$

$<10.0$

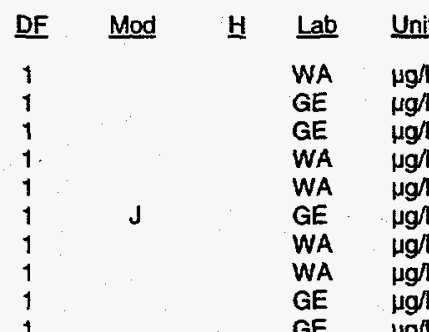

GE $\mu g /$

GE HgL

WA $\mu g / L$

GE $\mu g /$

WA $\mu g /$

GE $\quad \mu g / L$

WA $\mu g h$.

WA

GE $\mu g / L$

WA $\mu g /$ L

GE $\mu g / L$

$\mu g / L$
$\mu g / L$

$\mu g /$.

$\mu g /$

$\mu g /$.

$\mu g /$.

$\mu g / L$

$\mu g / h$

$\mu g / L$

$\mu g h$

$\mu g / h$.

$\mu g / L$

$\mu g /$

$\mathrm{g}$ gh

$\mu g / 2$

Hgh

$\mu g / L$

$\mu g / L$

$\mu g /$

$\mu g / L$

$\mu g /$

$\mu g h$

$\mu g /$

$\mu g / L$

$\mu g / L$

$\mu g /$

$\mu g / L$
$\mu g /$

$\mu g /$.

$\mu g h$

$\mu g /$

$\mu g h$

$\mu g /$

$\mu \mathrm{g}$ L

$\mu g h$

$\mu \mathrm{g} / \mathrm{L}$

$\mu g / L$

$\mu g / 2$

$\mu g^{\prime \prime}$

$\mu g /$

$\mu g h$

$\mu g /$

$\mu \mathrm{gh}$.

$\mu g / 2$

$\mu g h$

$\mu \mathrm{g} / \mathrm{L}$

$\mu \mathrm{g} / \mathrm{L}$

ugh

Note: Modifiers are listed and detailed in Appendix D, Key to Reading the Tables.

$-=$ exceeded holding time. 
Well LFW 43C (cont.)

Parameter

1,3-Dinitrobenzene

2,4-Dinitrophenol

2,4-Dinitrophenol

2,4-Dinitrophenol

2,4-Dinitrotoluene

2,4-Dinitrotoluene

2,6-Dinitrotoluene

2,6-Dinitrotoluene

2,6-Dinitrotoluene

Di-n-octyl phthalate

Di-n-octyl phthalate

Di-n-octyl phthalate

1,4-Dioxane

1,4-Dioxane

1,4-Dioxane

Diphenylamine

Diphenylamine

Diphenylamine

Disulfoton

Disulfoton

Disultoton

Endosulfan sulfate

Endosulfan sulfate

Endosutfan I

Endosulfan I

Endosulfan II

Endosulfan II

Endrin

Endrin

Endrin aldehyde

Endrin aldehyde

Ethyl methacrylate

Ethyl methacrylate

Ethyl methacrylate

Ethyl methanesulfonate

Ethyl methanesulfonate

Ethyl methanesulfonate

Ethylbenzene

Ethylbenzene

Famphur

Famphur

Famphur

Fluoranthene

Fluoranthene

Fluoranthene

Fluorene

Fluorene

Fluorene

Heptachlor

Heptachlor

Heptachlor epoxide

Heptachlor epoxide

Heptachlorodibenzo-p-dioxins

Heptachlorodibenzo-p-dioxins

Heptachlorodibenzo-p-furans

Heptachlorodibenzo-p-furans

Hexachlorobenzene

Hexachiorobenzene

Hexachiorobenzene

Hexachlorobutadiene

Hexachlorobutadiene

Hexachlorobutadiene

Hexachlorobutadiene

Hexachlorocyclopentadiene

Hexachlorocyclopentadiene

Hexachlorocyclopentadiene

Hexachlorodibenzo-p-dioxins

Hexachlorodibenzo-p-dioxins

Hexachlorodibenzo-p-furans

Hexachlorodibenzo-p-furans

Hexachloroethane

Hexachloroethane

Hexachloroethane

\begin{tabular}{|c|c|c|c|c|}
\hline 2Q96 & DF & Mod & H & Lab \\
\hline$<10.0$ & 1 & & & WA \\
\hline$<10.0$ & 1 & & & GE \\
\hline$<10.0$ & 1 & & & GE \\
\hline$<50.0$ & 1. & & & WA \\
\hline$<0.100$ & 1 & & & GE \\
\hline$<10.0$ & 1 & & & WA \\
\hline$<0.100$ & 1 & & & $\mathrm{GE}$ \\
\hline$<0.100$ & 1 & & & GE \\
\hline$<10.0$ & $i$ & & & WA \\
\hline$<1.00$ & 1 & & & GE \\
\hline$<1.00$ & 1 & & & GE \\
\hline$<10.0$ & 1 & & & WA \\
\hline$<10.0$ & 1 & & & GE \\
\hline$<10.0$ & 1 & & & $\overline{G E}$ \\
\hline$<10.0$ & 1 & & & WA \\
\hline$<10.0$ & 1 & & & GE \\
\hline$<10.0$ & 1 & & & GE \\
\hline$<10.0$ & 1 & & & WA \\
\hline$<1.00$ & 1 & & & GE \\
\hline$<1.00$ & 1 & & & GE \\
\hline$<0.206$ & 1 & & & WA \\
\hline$<0.0495$ & 1 & & & $\mathrm{GE}$ \\
\hline$<0.100$ & 1 & & & WA \\
\hline$<0.0248$ & 1 & & & GE \\
\hline$<0.0500$ & 1 & & & WA \\
\hline$<0.0495$ & 1 & & & GE \\
\hline$<0.100$ & 1 & & & WA \\
\hline$<0.0248$ & 1 & & & GE \\
\hline$<0.100$ & 1 & & & WA \\
\hline$<0.0619$ & 1 & & & $\mathrm{GE}$ \\
\hline$<0.100$ & 1 & & & WA \\
\hline$<10.0$ & 1 & & & $\mathrm{GE}$ \\
\hline$<10.0$ & 1 & & & GE \\
\hline$<10.0$ & 1 & & & WA \\
\hline$<10.0$ & 1 & & & GE \\
\hline$<10.0$ & 1 & & & GE \\
\hline$<10.0$ & 1 & & & WA \\
\hline$<2.00$ & 1 & & & GE \\
\hline$<5.00$ & 1 & & & WA \\
\hline$<10.0$ & 1 & & & GE \\
\hline$<10.0$ & 1 & & & GE \\
\hline$<1.03$ & 1 & & & WA \\
\hline$<1.00$ & 1 & & & GE \\
\hline$<1.00$ & $i$ & & & GE \\
\hline$<10.0$ & 1 & & & WA \\
\hline$<1.00$ & 1 & & & GE \\
\hline$<1.00$ & 1 & & & $\overline{G E}$ \\
\hline$<10.0$ & 1 & & & WA \\
\hline$<0.0248$ & 1 & & & GE \\
\hline$<0.0500$ & 1 & & & WA \\
\hline$<0.0248$ & 1 & & & GE \\
\hline$<0.0500$ & 1 & & & WA \\
\hline$<0.0100$ & 1 & & & $\mathrm{GE}$ \\
\hline$<0.00160$ & 1 & & & WA \\
\hline$<0.0100$ & 1 & & & GE \\
\hline$<0.00230$ & 1 & & & WA \\
\hline$<0.100$ & 1 & & & GE \\
\hline$<0.100$ & 1 & & & $\overline{G E}$ \\
\hline$<10.0$ & $i$ & & & WA \\
\hline$<1.00$ & 1 & & & $\mathrm{GE}$ \\
\hline$<1.00$ & 1 & & & GE \\
\hline$<5.00$ & 1 & & & WA \\
\hline$<10.0$ & 1 & & & WA \\
\hline$<1.00$ & 1 & & & GE \\
\hline$<1.00$ & 1 & & & GE \\
\hline$<10.0$ & 1 & & & WA \\
\hline$<0.0100$ & 1 & & & $\mathrm{GE}$ \\
\hline$<0.00270$ & 1 & & & WA \\
\hline$<0.0100$ & $i$ & & & $\mathrm{GE}$ \\
\hline$<0.00230$ & 1 & & & WA \\
\hline$<0.100$ & 1 & & & GE \\
\hline$<0.100$ & i & & & $\mathrm{GE}$ \\
\hline$<10.0$ & 1 & & & WA \\
\hline
\end{tabular}

Note: Modifiers are listed and detailed in Appendix D, Key to Reading the Tables.

- exceeded holding time. 
Well LFW 43C (cont.)

\section{Parameter}

Hexachlorophene

Hexachlorophene

Hexachlorophene

Hexachloropropene

Hexachloropropene

Hexachloropropene

2-Hexanone

2-Hexanone

1,2,3,4,6,7,8-HPCDD

$1,2,3,4,6,7,8-H P C D D$

$1,2,3,4,6,7,8$-HPCDF

$1,2,3,4,6,7,8-H P C D F$

$1,2,3,4,7,8-\mathrm{HXCDD}$

$1,2,3,4,7,8-H X C D D$

$1,2,3,4,7,8-H X C D D$

$1,2,3,4,7,8-\mathrm{HXCDF}$

$1,2,3,4,7,8-\mathrm{HXCDF}$

$1,2,3,4,7,8-H X C D F$

Indeno(1,2,3-c,d)pyrene

Indeno(1,2,3-c,d)pyrene

Indeno $(1,2,3-c, d)$ pyrene

lodomethane (Methyl iodide)

lodomethane (Methyl iodide)

Isobutyl alcohol

Isobutyl alcohol

Isodrin

Isodrin

Isodrin

Isophorone

Isophorone

Isophorone

Isopropylbenzene

p-Isopropyltoluene

Isosafrole

Isosafrole

Isosafrole

Kepone

Kepone

Kepone

Lead, total recoverable

Lead total recoverable

Lead, total recoverable

Lindane

Lindane

Mercury, total recoverable

Mercury, total recoverable

Methacrylonitrile

Methacrylonitrile

Methapyrilene

Methapyriene

Methapyrilene

Methoxychlor

Methoxychlor

2-Methyl-4,6-dinitropheno

2-Methyl-4,6-dinitropheno

2-Methyl-4,6-dinitropheno

Methyl ethyl ketone

Methyl ethyl ketone

Methyi isobutyl ketone

Methyl isobutyl ketone

Methyl methacrylate

Methyl methacrylate

Methyl methanesulfonate

Methyl methanesulfonate

Methyl methanesulfonate

3-Methylcholanthrene

3-Methylcholanthren

3-Methylcholanthren

2-Methylnaphthalene

2-Methylnaphthalene

2-Methylnaphthalene

Naphthalene

\begin{tabular}{|c|c|c|c|c|}
\hline 2096 & DF & Mod & $\underline{H}$ & $\underline{\text { Lab }}$ \\
\hline$<80.0$ & 1 & & & GE \\
\hline$<80.0$ & 1 & & & GE \\
\hline$<90.0$ & 1 & & & WA \\
\hline$<10.0$ & 1. & & & GE \\
\hline$<10.0$ & 1 & & & GE \\
\hline$<10.0$ & 1 & & & WA \\
\hline$<10.0$ & 1 & & & GE \\
\hline$<10.0$ & 1 & & & WA \\
\hline$<0.0100$ & 1 & & & GE \\
\hline$<0.00160$ & 1 & & & WA \\
\hline$<0.0100$ & 1 & & & GE \\
\hline$<0.00210$ & 1 & & & WA \\
\hline$<0.0100$ & 1 & & & GE \\
\hline$<0.00210$ & 1 & & & WA \\
\hline$<0.00270$ & 1 & & & WA \\
\hline$<0.0100$ & 1 & & & GE \\
\hline$<0.00170$ & 1 & & & WA \\
\hline$<0.00220$ & 1 & & & WA \\
\hline$<0.100$ & 1 & & & GE \\
\hline$<0.100$ & 1 & & & GE \\
\hline$<10.0$ & 1 & & & WA \\
\hline$<10.0$ & 1 & & & GE \\
\hline$<5.00$ & 1 & & & WA \\
\hline$<20.0$ & 1 & & & GE \\
\hline$<50.0$ & 1 & & & WA \\
\hline$<10.0$ & 1 & & & GE \\
\hline$<10.0$ & 1 & & & GE \\
\hline$<0.100$ & 1 & & & WA \\
\hline$<1.00$ & 1 & & & GE \\
\hline$<1.00$ & 1 & & & GE \\
\hline$<10.0$ & 1 & & & WA \\
\hline$<5.00$ & 1 & & & WA \\
\hline$<5.00$ & 1 & & & WA \\
\hline$<10.0$ & 1 & & & $\mathrm{GE}$ \\
\hline$<10.0$ & 1 & & & $\overline{\mathbf{G E}}$ \\
\hline$<10.0$ & 1 & & & WA \\
\hline$<10.0$ & 1 & & & $\mathrm{GE}$ \\
\hline$<10.0$ & 1 & & & GE \\
\hline$<0.500$ & 1 & & & WA \\
\hline$<5.00$ & 1 & & & GE \\
\hline$<47.0$ & 1 & & & WA \\
\hline$<47.0$ & 1 & & & WA \\
\hline$<0.0124$ & 1 & & & GE \\
\hline$<0.0500$ & 1 & & & WA \\
\hline$<0.200$ & 1 & & & GE \\
\hline$<0.700$ & 1 & & & WA \\
\hline$<10.0$ & 1 & & & GE \\
\hline$<20.0$ & 1 & & & WA \\
\hline$<10.0$ & 1 & & & GE \\
\hline$<10.0$ & 1 & & & GE \\
\hline$<10.0$ & 1 & & & WA \\
\hline$<0.248$ & 1 & & & GE \\
\hline$<0.500$ & 1 & & & WA \\
\hline$<10.0$ & 1 & & & GE \\
\hline$<10.0$ & 1 & & & $\mathrm{GE}$ \\
\hline$<50.0$ & 1 & & & WA \\
\hline$<5.00$ & 1 & & & GE \\
\hline$<10.0$ & 1 & & & WA \\
\hline$<10.0$ & 1 & & & $\mathrm{GE}$ \\
\hline$<10.0$ & 1 & & & WA \\
\hline$<10.0$ & 1 & & & $\mathrm{GE}$ \\
\hline$<10.0$ & $i$ & & & WA \\
\hline$<10.0$ & 1 & & & $\mathrm{GE}$ \\
\hline$<10.0$ & 1 & & & GE \\
\hline$<10.0$ & 1 & & & WA \\
\hline$<10.0$ & 1 & & & GE \\
\hline$<10.0$ & 1 & & & GE \\
\hline$<10.0$ & 1 & & & WA \\
\hline$<1.00$ & 1 & & & GE \\
\hline$<1.00$ & 1 & & & GE \\
\hline$<10.0$ & 1 & & & WA \\
\hline$<1.00$ & 1 & & & GE \\
\hline
\end{tabular}

Note: Modifiers are listed and detailed in Appendix D, Key to Reading the Tables.

$\bullet=$ exceeded holding time. 
Well LFW 43C (cont.)

\section{Parameter}

Naphthalene

Naphthalene

Naphthalene

1,4-Naphthoquinone

1,4-Naphthoquinone

1,4-Naphthoquinone

1-Naphthylamine

1-Naphthylamine

1-Naphthylamine

2-Naphthylamine

2-Naphthylamine

2-Naphthylamine

Nickel, total recoverable

Nickel, total recoverable

Nickel, total recoverable

m-Nitroaniline

m-Nitroaniline

m-Nitroaniline

o-Nitroaniline

o-Nitroaniline

o-Nitroaniline

p-Nitroaniline

p-Nitroaniline

p-Nitroaniline

Nitrobenzene

Nitrobenzene

Nitrobenzene

2-Nitrophenol

2-Nitrophenol

2-Nitrophenol

4-Nitropheno

4-Nitropheno

4-Nitropheno

4-Nitroquinoline-1-oxide

4-Nitroquinoline-1-oxide

$\mathrm{N}$-Nitrosodi-n-butylamine

$\mathrm{N}$-Nitrosodi-n-butylamine

$\mathrm{N}$-Nitrosodi-n-butylamine

$\mathrm{N}$-Nitrosodiethylamine

$\mathrm{N}$-Nitrosodiethylamine

$\mathrm{N}$-Nitrosodiethylamine

$\mathrm{N}$-Nitrosodimethylamine

N-Nitrosodimethylamine

$\mathrm{N}$-Nitrosodimethylamine

N-Nitrosodiphenylamine

N-Nitrosodiphenylamine

N-Nitrosodiphenylamine

N-Nitrosodipropylamine

N-Nitrosodipropylamine

$\mathrm{N}$-Nitrosomethylethylamine

$\mathrm{N}$-Nitrosomethylethylamine

N-Nitrosomethylethylamine

N-Nitrosomorpholine

$\mathrm{N}$-Nitrosomorpholine

N-Nitrosomorpholine

$\mathrm{N}$-Nitrosopiperidine

$\mathrm{N}$-Nitrosopiperidine

$\mathrm{N}$-Nitrosopiperidine

N-Nitrosopyrrolidine

$\mathrm{N}$-Nitrosopyrrolidine

$\mathrm{N}$-Nitrosopyrrolidine

5-Nitro-o-toluidine

5-Nitro-o-toluidine

5-Nitro-o-toluidine

Octachlorodibenzo-p-dioxin

Octachlorodibenzo-p-dioxin

Octachlorodibenzo-p-furan

Octachlorodibenzo-p-furan

Parathion

Parathion ethyl

Parathion methyl

Parathion methyl

PCB 1016

\begin{tabular}{|c|c|c|c|c|c|}
\hline$\underline{2096}$ & $\underline{D F}$ & Mod & H & Lab & $\underline{\text { Unit }}$ \\
\hline$<1.00$ & 1 & & & GE & $\mu g / L$ \\
\hline$<5.00$ & 1 & & & WA & $\mu g / L$ \\
\hline$<10.0$ & 1 & & & WA & $\mu g / L$ \\
\hline$<10.0$ & 1. & & & GE & $\mu g /$ \\
\hline$<10.0$ & 1 & & & GE & $\mu g /$ \\
\hline$<10.0$ & 1 & & & WA & $\mu g / L$ \\
\hline$<10.0$ & 1 & & & GE & $\mu g / L$ \\
\hline$<10.0$ & 1 & & & GE & $\mu g /$. \\
\hline$<10.0$ & 1 & & & WA & $\mu \mathrm{g} / \mathrm{L}$ \\
\hline$<10.0$ & 1 & & & GE & $\mu g / L$ \\
\hline$<10.0$ & 1 & & & GE & $\mu g /$ \\
\hline$<10.0$ & 1 & & & WA & $\mu g / L$ \\
\hline$<10.0$ & 1 & & & GE & $\mu g h$ \\
\hline$<26.0$ & 1 & & & WA & $\mu \mathrm{g} / \mathrm{L}$ \\
\hline$<26.0$ & 1 & & & WA & $\mu g / L$ \\
\hline$<1.00$ & 1 & & & GE & $\mu g /$ \\
\hline$<1.00$ & 1 & & & $\mathrm{GE}$ & $\mu g /$ \\
\hline$<50.0$ & 1 & & & WA & $\mu g /$. \\
\hline$<1.00$ & 1 & & & GE & $\mu g /$ \\
\hline$<1.00$ & 1 & & & $\mathrm{GE}$ & $\mu g /$ \\
\hline$<50.0$ & 1 & & & WA & $\mu g / L$ \\
\hline$<1.00$ & 1 & & & GE & $\mu g /$ \\
\hline$<1.00$ & 1 & & & GE & $\mu g /$ \\
\hline$<50.0$ & 1 & & & WA & $\mu g / L$ \\
\hline$<1.00$ & 1 & & & GE & $\mu g / L$ \\
\hline$<1.00$ & 1 & & & $\mathrm{GE}$ & $\mu g /$ \\
\hline$<10.0$ & 1 & & & WA & $\mu g / L$ \\
\hline$<1.00$ & 1 & & & GE & $\mu g / L$ \\
\hline$<1.00$ & 1 & & & $\mathrm{GE}$ & $\mu g / L$ \\
\hline$<10.0$ & 1 & & & WA & $\mu g / L$ \\
\hline$<1.00$ & 1 & J & & GE & $\mu g / L$ \\
\hline$<1.00$ & 1 & $\mathbf{J}$ & & GE & jgh \\
\hline$<50.0$ & 1 & & & WA & $\mu g /$ \\
\hline$<10.0$ & 1 & & & GE & $\mu g / L$ \\
\hline$<20.0$ & 1 & & & WA & $\mu g / L$ \\
\hline$<10.0$ & 1 & & & GE & $\mu g / L$ \\
\hline$<10.0$ & 1 & & & GE & $\mu g / L$ \\
\hline$<10.0$ & 1 & & & WA & $\mu g / L$ \\
\hline$<10.0$ & 1 & & & GE & $\mu g^{\prime} L$ \\
\hline$<10.0$ & $t$ & & & GE & $\mu g /$ \\
\hline$<10.0$ & 1 & & & WA & $\mu g /$ \\
\hline$<1.00$ & 1 & & & GE & $\mu g /$ \\
\hline$<1.00$ & 1 & & & GE & $\mu g / L$ \\
\hline$<10.0$ & 1 & & & WA & $\mu g / L$ \\
\hline$<1.00$ & 1 & & & $\mathrm{GE}$ & $\mu \mathrm{g} / \mathrm{L}$ \\
\hline$<1.00$ & 1 & & & GE & $\mu g / L$ \\
\hline$<10.0$ & 1 & & & WA & $\mu g / L$ \\
\hline$<1.00$ & 1 & & & GE & $\mu g / L$ \\
\hline$<10.0$ & 1 & & & WA & $\mu g / L$ \\
\hline$<10.0$ & 1 & & & GE & $\mu g / L$ \\
\hline$<10.0$ & 1 & & & GE & $\mu \mathrm{g} / \mathrm{L}$ \\
\hline$<10.0$ & 1 & & & WA & $\mu g / L$ \\
\hline$<10.0$ & 1 & & & GE & $\mu g /$ \\
\hline$<10.0$ & 1 & & & GE & $\mu g / L$ \\
\hline$<10.0$ & 1 & & & WA & $\mu g / L$ \\
\hline$<10.0$ & 1 & & & $\mathrm{GE}$ & $\mu g / L$ \\
\hline$<10.0$ & 1 & & & GE & $\mu g / L$ \\
\hline$<50.0$ & 1 & & & WA & $\mu g /$ \\
\hline$<10.0$ & 1 & & & $\mathrm{GE}$ & $\mu g /$ \\
\hline$<10.0$ & 1 & & & GE & $\mu g /$ \\
\hline$<10.0$ & 1 & & & WA & $\mu g /$ \\
\hline$<10.0$ & 1 & & & GE & $\mu g / L$ \\
\hline$<10.0$ & 1 & & & $\mathrm{GE}$ & $\mu g /$ \\
\hline$<10.0$ & 1 & & & WA & $\mu g /$ \\
\hline$<0.0100$ & 1 & & & GE & $\mu \mathrm{g} / \mathrm{L}$ \\
\hline$<0.00250$ & 1 & & & WA & $\mu g / h$ \\
\hline$<0.0100$ & 1 & & & GE & $\mu g / L$ \\
\hline$<0.00150$ & 1 & & & WA & $\mu g h$ \\
\hline$<0.0248$ & 1 & & & GE & $\mu g /$ \\
\hline$<0.206$ & 1 & & & WA & $\mu g / t$ \\
\hline$<0.0248$ & 1 & & & GE & $\mu g /$ \\
\hline$<0.206$ & 1 & & & WA & $\mu g /$ \\
\hline$<0.125$ & 1 & & & GE & $\mu g /$ \\
\hline
\end{tabular}

Note: Modifiers are listed and detailed in Appendix D, Key to Reading the Tables.

- = exceeded holding time. 
Well LFW 43C (cont.)

\begin{tabular}{|c|c|c|c|c|c|c|}
\hline Parameter & 2096 & DF & Mod & $\underline{H}$ & $\underline{\text { Lab }}$ & Unit \\
\hline PCB 1016 & $<0.0619$ & 1 & & & GE & $\mu \mathrm{g} / \mathrm{L}$ \\
\hline PCB 1016 & $<1.00$ & 1 & & & WA & \\
\hline PCB 1221 & $<0.125$ & 1 & & & $\mathrm{GE}$ & $\mu \mathrm{g}$ \\
\hline PCB 1221 & $<0.0619$ & 1. & & & GE & \\
\hline PCB 1221 & $<2.00$ & 1 & & & WA & $\mu g$ \\
\hline PCB 1232 & $<0.125$ & 1 & & & GE & $\mu \mathrm{g}$ \\
\hline РСB 1232 & $<0.0619$ & 1 & & & GE & \\
\hline PCB 1232 & $<1.00$ & 1 & & & WA & $\mu g$ \\
\hline РCB 1242 & $<0.125$ & 1 & & & GE & \\
\hline PCB 1242 & $<0.0619$ & 1 & & & GE & \\
\hline PCB 1242 & $<1.00$ & 1 & & & WA & \\
\hline PCB 1248 & $<0.125$ & 1 & & & GE & \\
\hline PCB 1248 & $<0.0619$ & 1 & & & GE & \\
\hline PCB 1248 & $<1.00$ & 1 & & & WA & \\
\hline PCB 1254 & $<0,125$ & 1 & & & GE & \\
\hline PCB 1254 & $<0.0619$ & 1 & & & GE & \\
\hline РCB 1254 & $<1.00$ & 1 & & & WA & \\
\hline PCB 1260 & $<0.0619$ & 1 & & & $\mathrm{GE}$ & \\
\hline PCB 1260 & $<0.125$ & 1 & $\mathrm{~J}$ & & GE & \\
\hline PCB 1260 & $<1.00$ & 1 & & & WA & \\
\hline $1,2,3,7,8-P C D D$ & $<0.0100$ & 1 & & & GE & \\
\hline $1,2,3,7,8-P C D D$ & $<0.00300$ & 1 & & & WA & \\
\hline $1,2,3,7,8-P C D F$ & $<0.0100$ & 1 & & & GE & \\
\hline $1,2,3,7,8-P C D F$ & $<0.00210$ & 1 & & & WA & \\
\hline Pentachlorobenzene & $<10.0$ & 1 & & & GE & \\
\hline Pentachlorobenzene & $<10.0$ & 1 & & & $\overline{G E}$ & \\
\hline Pentachlorobenzene & $<10.0$ & 1 & & & WA & \\
\hline Pentachlorodibenzo-p-dioxins & $<0.0100$ & 1 & & & GE & \\
\hline Pentachlorodibenzo-p-dioxins & $<0.00300$ & 1 & & & WA & \\
\hline Pentachlorodibenzo-p-furans & $<0.0100$ & 1 & & & GE & \\
\hline Pentachlorodibenzo-p-furans & $<0.00210$ & 1 & & & WA & \\
\hline Pentachioroethane & $<10.0$ & 1 & & & GE & \\
\hline Pentachloroethane & $<10.0$ & 1 & & & GE & \\
\hline Pentachloroethane & $<10.0$ & 1 & & & WA & \\
\hline Pentachloronitrobenzene & $<10.0$ & 1 & & & GE & \\
\hline Pentachloronitrobenzene & $<10.0$ & 1 & & & GE & \\
\hline Pentachloronitrobenzene & $<50.0$ & 1 & & & WA & \\
\hline Pentachlorophenol & $<1.00$ & 1 & & & $\mathrm{GE}$ & \\
\hline Pentachlorophenol & $<50.0$ & 1 & & & WA & \\
\hline Phenacetin & $<10.0$ & 1 & & & $\mathrm{GE}$ & \\
\hline Phenacetin & $<10.0$ & 1 & & & GE & \\
\hline Phenacetin & $<10.0$ & 1 & & & WA & \\
\hline Phenanthrene & $<1.00$ & 1 & & & GE & \\
\hline Phenanthrene & $<1.00$ & 1 & & & GE & \\
\hline Phenanthrene & $<10.0$ & 1 & & & WA & \\
\hline Phenol & $<1.00$ & 1 & $J$ & & $\mathrm{GE}$ & \\
\hline Phenol & $<10.0$ & 1 & & & WA & \\
\hline p-Phenylenediamine & $<10.0$ & 1 & & & GE & \\
\hline p-Phenylenediamine & $<10.0$ & 1 & & & GE & \\
\hline p-Phenylenediamine & $<10.0$ & 1 & & & WA & \\
\hline Phorate & $<0.0248$ & 1 & & & $\mathrm{GE}$ & \\
\hline Phorate & $<0.206$ & 1 & & & WA & \\
\hline 2-Picoline & $<1.00$ & 1 & & & GE & \\
\hline 2-Picoline & $<1.00$ & 1 & & & $\mathrm{GE}$ & \\
\hline 2-Picoline & $<10.0$ & 1 & & & WA & \\
\hline Pronamid & $<10.0$ & $t$ & & & GE & \\
\hline Pronamid & $<10.0$ & 1 & & & $\mathrm{GE}$ & \\
\hline Pronamid & $<10.0$ & 1 & & & WA & \\
\hline Propionitrile & $<20.0$ & 1 & & & GE & \\
\hline Propionitrile & $<20.0$ & 1 & & & WA & \\
\hline n-Propylbenzene & $<5.00$ & 1 & & & WA & \\
\hline Pyrene & $<1.00$ & 1 & & & GE & \\
\hline Pyrene & $<10.0$ & 1 & & & WA & \\
\hline Pyridine & $<1.00$ & 1 & & & $\mathrm{GE}$ & \\
\hline Pyridine & $<1.00$ & 1 & & & GE & \\
\hline Pyridine & $<10.0$ & 1 & & & WA & \\
\hline Safrole & $<10.0$ & 1 & & & GE & \\
\hline Safrole & $<10.0$ & 1 & & & GE & \\
\hline Safrole & $<10.0$ & 1 & & & WA & \\
\hline Selenium, total recove & $<5.00$ & 1 & & & GE & \\
\hline Selenium, total recoverable & $<66.0$ & 1 & & & WA & \\
\hline Selenium, total recoverable & $<66.0$ & 1 & & & WA & \\
\hline Silver total recoverable & $<2.00$ & 1 & & & & \\
\hline
\end{tabular}

Note: Modifiers are listed and detailed in Appendix D, Key to Reading the Tables.

- = exceeded holding time. 
Well LFW $43 C$ (cont.)

Parameter

Silver, total recoverable
Silver, total recoverable

Styrene

Styrene

Sulfide

Sulfide

Sulfotepp

Sulfotepp

Sulfotepp

2,4,5-T

$2,4,5-\mathrm{T}$

2,4,5-T

2,4,5-T

2,3,7,8-TCDD

2,3,7,8-TCDD

2,3,7,8-TCDF

2,3,7,8-TCDF

1,2,4,5-Tetrachlorobenzene

1,2,4,5-Tetrachlorobenzene

1,2,4,5-Tetrachlorobenzene

Tetrachlorodibenzo- $p$-dioxins

Tetrachlorodibenzo-p-dioxins

Tetrachlorodibenzo-p-furans

Tetrachlorodibenzo-p-furans

$1, t, 1,2$-Tetrachloroethane

1,1,1,2-Tetrachloroethane

1,1,2,2-Tetrachloroethane

$1,1,2,2-$ Tetrachloroethane

Tetrachloroethylene

Tetrachloroethylene

2,3,4,6-Tetrachlorophenol

2,3,4,6-Tetrachloropheno

2,3,4,6-Tetrachlorophenol

Thallium, total recoverable

Thallium, total recoverable

Thallium, total recoverable

Thionazin

Thionazin

Thionazin

Tin, total recoverable

Tin, total recoverable

Tin, total recoverable

Toluene

Toluene

o-Toluidine

o-Toluidine

o-Toluidine

Toxaphene

Toxaphene

2,4,5-TP (Silvex)

2,4,5-TP (Silvex)

2,4,5-TP (Silvex)

2,4,5-TP (Silvex)

1,2,3-Trichlorobenzene

1,2,4-Trichlorobenzene

1,2,4-Trichlorobenzene

1,2,4-Trichlorobenzene

1,1,1-Trichloroethane

1,1,1-Trichloroethane

1,1,2-Trichloroethane

Trichloroethylene

Trichloroethylene

Trichiorofluoromethane

Trichlorofluoromethane

2,4,5-Trichlorophenol

2,4,5-Trichloropheno]

2,4,5-Trichlorophenol

2,4,6-Trichlorophenol

2,4,6-Trichlorophenol

2,4,6-Trichlorophenol

1,2,3-Trichloropropane

1,2,3-Trichloropropane

Trichlorotrifluoroethane

\begin{tabular}{|c|c|c|c|c|c|}
\hline 2096 & DF & Mod & 브 & $\underline{\text { Lab }}$ & Unit \\
\hline$<5.00$ & 1 & & & WA & $\mu g / L$. \\
\hline$<5.00$ & 1 & & & WA & $\mu g / L$ \\
\hline$<2.00$ & 1 & & & GE & $\mu g / L$ \\
\hline$<5.00$ & 1 & & & WA & $\mu g /$ \\
\hline$<100$ & 1 & & & GE & $\mu g / L$ \\
\hline$<210$ & 1 & & & WA & $\mu g / L$ \\
\hline$<0.400$ & 1 & & & GE & $\mu g /$ \\
\hline$<0.400$ & 1 & & & $\mathrm{GE}$ & $\mu g / L$ \\
\hline$<0.206$ & 1 & & & WA & $\mu g / L$ \\
\hline$<0.0490$ & 1 & & & GE & $\mu g / L$ \\
\hline$<0.0495$ & 1 & & & GE & $\mu g /$ \\
\hline$<0.510$ & 1 & $J$ & & WA & $\mu g / L$ \\
\hline$<0.505$ & 1 & $J$ & $\bullet$ & WA & $\mu g /$ \\
\hline$<0.0100$ & 1 & & & $\mathrm{GE}$ & $\mu g / L$ \\
\hline$<0.00190$ & 1 & & & WA & $\mu g /$ \\
\hline$<0.0100$ & 1 & & & $\mathrm{GE}$ & $\mu g / L$ \\
\hline$<0.00190$ & 1 & & & WA & $\mu g /$ \\
\hline$<0.400$ & 1 & & & $\mathrm{GE}$ & $\mu g / L$ \\
\hline$<0.400$ & 1 & & & GE & $\mu g /$ \\
\hline$<10.0$ & 1 & & & WA & $\mu g \Omega$. \\
\hline$<0.0100$ & 1 & & & GE & $\mu g / 2$ \\
\hline$<0.00190$ & 1 & & & WA & $\mu g / L$ \\
\hline$<0.0100$ & 1 & & & GE & $\mu g / L$ \\
\hline$<0.00190$ & 1 & & & WA & $\mu g /$ \\
\hline$<2.00$ & 1 & & & GE & $\mu g /$. \\
\hline$<5.00$ & 1 & & & WA & $\mu g / L$ \\
\hline$<2.00$ & 1 & & & GE & $\mu g / L$ \\
\hline$<5.00$ & 1 & & & WA & $\mu g / L$ \\
\hline$<2.00$ & 1 & & & $\mathrm{GE}$ & $\mu g / L$ \\
\hline$<5.00$ & 1 & & & WA & $\mu g h$ \\
\hline$<10.0$ & 1 & & & GE & $\mu g /$ \\
\hline$<10.0$ & 1 & & & GE & $\mu g / L$ \\
\hline$<10.0$ & 1 & & & WA & $\mu g / L$ \\
\hline$<5.00$ & 1 & & & $\mathrm{GE}$ & $\mathrm{g} / \mathrm{h}$ \\
\hline$<55.0$ & 1 & & & WA & $\mu g / L$ \\
\hline$<55.0$ & 1 & & & WA & $\mu g / L$ \\
\hline$<10.0$ & 1 & & & GE & $\mu g / L$ \\
\hline$<10.0$ & $t$ & & & GE & $\mu g /$ \\
\hline$<0.206$ & 1 & & & WA & $\mu g / L$ \\
\hline$<50.0$ & 1 & & & $\mathrm{GE}$ & $\mu g / L$ \\
\hline$<70.0$ & 1 & & & WA & $\mu g /$ \\
\hline$<70.0$ & 1 & & & WA & $\mu g / L$ \\
\hline$<2.00$ & 1 & & & GE & $\mu g h$ \\
\hline$<5.00$ & 1 & & & WA & $\mu g / L$ \\
\hline$<10.0$ & 1 & & & GE & $\mu g / L$ \\
\hline$<10.0$ & 1 & & & GE & $\mu g / L$ \\
\hline$<10.0$ & 1 & & & WA & $\mu g / L$ \\
\hline$<0.495$ & 1 & & & GE & $\mu g / L$ \\
\hline$<5.00$ & 1 & & & WA & jugl \\
\hline$<0.0245$ & 1 & $J$ & & GE & $\mu g / L$ \\
\hline$<0.0248$ & 1 & $\mathbf{J}$ & & GE & $\mu g / L$ \\
\hline$<0.510$ & 1 & & & WA & $\mu g / L$ \\
\hline$<0.505$ & 1 & $\mathbf{J}$ & - & WA & $\mu g / L$ \\
\hline$<5.00$ & 1 & & & WA & $\mu g / L$ \\
\hline$<0.100$ & 1 & $J$ & & GE & $\mu g / L$ \\
\hline$<5.00$ & 1 & & & WA & $\mu g / L$ \\
\hline$<10.0$ & 1 & & & WA & $\mu g h$ \\
\hline$<2.00$ & 1 & & & GE & $\mu g / L$ \\
\hline$<5.00$ & 1 & & & WA & \\
\hline$<2.00$ & 1 & & & GE & $\mu g /$ \\
\hline$<2.00$ & 1 & & & GE & $\mu g / L$ \\
\hline$<5.00$ & 1 & & & WA & $\mu g / L$ \\
\hline$<2.00$ & 1 & & & GE & $\mu g /$ \\
\hline$<5.00$ & 1 & & & WA & $\mu g /$ \\
\hline$<1.00$ & 1 & & & GE & $\mu g / L$ \\
\hline$<1.00$ & 1 & & & GE & $\mu g h$ \\
\hline$<50.0$ & 1 & & & WA & $\mu g / L$ \\
\hline$<0.100$ & 1 & & & GE & $\mu g / L$ \\
\hline$<0.100$ & 1 & & & GE & $\mu g / L$ \\
\hline$<10.0$ & 1 & & & WA & $\mu g / L$ \\
\hline$<2.00$ & 1 & & & GE & $\mu g / L$ \\
\hline$<5.00$ & 1 & & & WA & $\mu g / L$ \\
\hline$<5.00$ & 1 & & & WA & \\
\hline
\end{tabular}

Note: Modifiers are listed and detailed in Appendix D, Key to Reading the Tables.

$\bullet=$ exceeded holding time. 
Well LFW 43C (cont.)

Parameter

$0,0,0$-Triethyl phosphorothioate $0,0,0$-Triethyl phosphorothioate 0 -Triethyl phosphorothioate 1,2,4-Trimethylbenzene

1,3,5-Trimethylbenzene

1,3,5-Trinitrobenzene

1,3,5-Trinitrobenzene

1,3,5-Trinitrobenzene

Vanadium, total recoverable

Vanadium, total recoverable

Vanadium, total recoverable

Vinyl acetate

Vinyl acetate

mip-Xylene

o-Xylene

Xylenes

Zinc, total recoverable

Zinc, total recoverable

Zinc, total recoverable

D
1
1
1
1
1
1
1
1
1
1
1
1
1
1
1
1
1
1
1

Mod

브 L

GE $\mu g /$

GE $\mu g /$

WA $\mu g \Omega$

WA $\mu g h$

WA $\mu g /$ L

GE $\mu g /$

WA $\mu g /$

v

$\mu g / L$

$\mu \mathrm{g} / \mathrm{L}$

$\mu g$ L

$\mu g /$

$\mu g /$ L

Hgh

$\mu g h$

$\mu g / L$

GE $\quad \mu g / L$

WA $\mu g / L$

WA $\mu g / \mathrm{h}$

Note: Modifiers are listed and detailed in Appendix D, Key to Reading the Tables.

$\bullet=$ exceeded holding time. 


\section{WELLL LFW 43C Replicate}

\section{SRS Coord.}

N86480.6

E45234.9

SAMPLE DATE

Lat/Longitude
$33.290830^{\circ} \mathrm{N}$
$81.715670^{\circ} \mathrm{W}$

Screen Zone Elevation

138.5-128.5 ft ms

$04 / 23 / 96$

FIELD DATA

Parameter

Water elevation

$\mathrm{pH}$

Sp. conductance

Water temperature

Alkalinity as $\mathrm{CaCO}_{3}$

Turbidity

Volume purged

Sampling code

\section{ANALYTICAL DATA}

\section{Parameter}

1,1-Dichloropropene

Acenaphthene

Acenaphthylene

Acenaphthylene

Acetone

Acetone

Acetonitrile (Methyl cyanide)

Acetonitrile (Methyl cyanide)

Acetophenone

Acetophenone

2-Acetylaminofluorene

2-Acetylaminofluorene

Acrolein

Acrolein

Acrylonitrile

Acrylonitrile

Aldrin

Aldnin

Aldrin

Allyl chiloride

Allyl chloride

4-Aminobiphenyl

4-Aminobiphenyl

Aniline

Aniline

Anthracene

Anthracene

Antimony, total recoverable

Antimony, total recoverable

Aramite

Aramite

Arsenic, total recoverable

Arsenic, total recoverable

Barium, total recoverable

Barium, total recoverable

Benzene

Benzene

alpha-Benzene hexachloride

alpha-Benzene hexachloride

alpha-Benzene hexachloride

beta-Benzene hexachloride

beta-Benzene hexachloride

beta-Benzene hexachloride

delta-Benzene hexachloride

delta-Benzene hexachloride

delta-Benzene hexachloride

Benzo(a)anthracene

Benzo(a)anthracene

Benzo(b)fiuoranthene

Benzo(b)fluoranthene

Benzo(k)fluoranthene $\underline{2096}$

165.9

25.0

5.4

$\frac{\text { Top of Casing }}{202.6 \mathrm{ft} \mathrm{msl}} \quad \frac{\text { Casing }}{4^{n} \text { PVC }} \quad \frac{\text { Pump }}{\mathrm{s}}$

$202.6 \mathrm{ft} \mathrm{msl}$
Screen Zone

Middle Steed Pond
Unit

ft msl

$\mathrm{pH}$

$\mu \mathrm{S} / \mathrm{cm}$

C

mgl

well vol

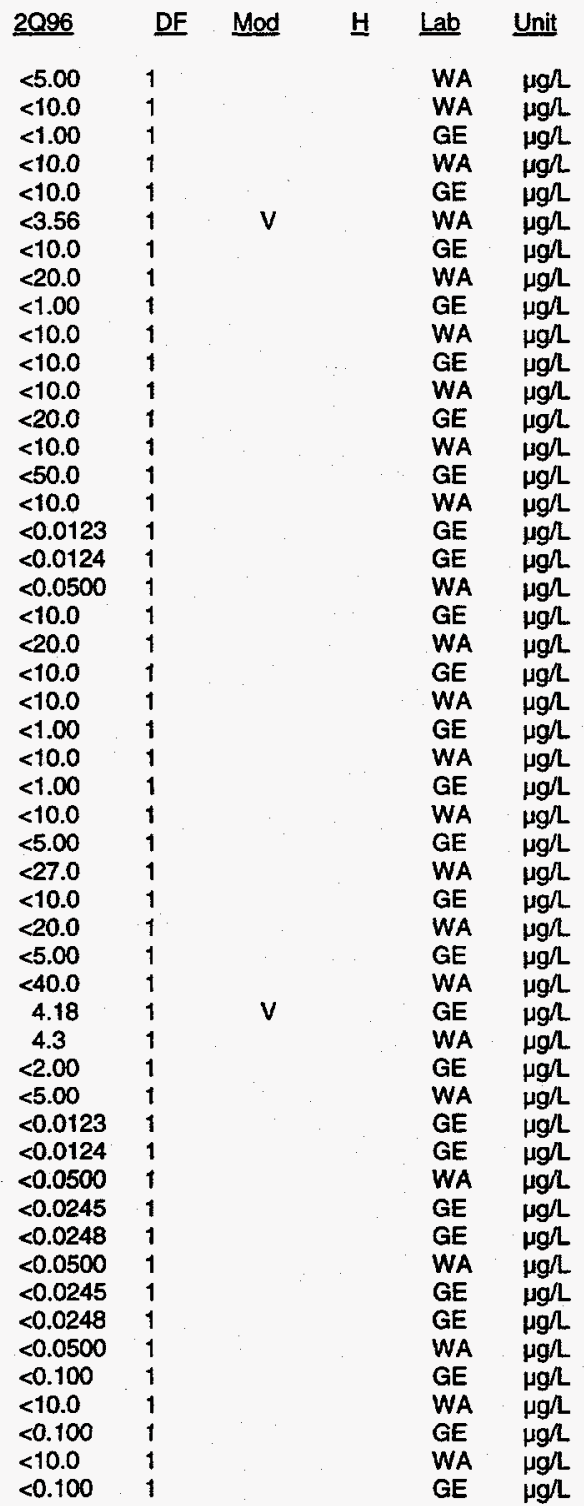

Note: Modifiers are listed and detailed in Appendix D, Key to Reading the Tables.

$\bullet=$ exceeded holding time. 


\begin{tabular}{|c|c|c|c|c|c|c|}
\hline Parameter & $\underline{2096}$ & $\underline{D F}$ & Mod & $\underline{\mathrm{H}}$ & $\underline{\text { Lab }}$ & Unit \\
\hline Benzo(k)fluoranthene & $<10.0$ & 1 & & & WA & $\mu g / L$ \\
\hline Benzoic acid & $<50.0$ & 1 & & & WA & $\mu g / L$ \\
\hline Benzo(g,h,i)perylene & $<1.00$ & 1 & & & GE & $\mu \mathrm{g} / \mathrm{L}$ \\
\hline Benzo $(\mathbf{g}, \mathrm{h}, \mathrm{i})$ perylene & $<10.0$ & 1. & & & WA & $\mu g / L$ \\
\hline Benzo(a)pyrene & $<0.100$ & 1 & & & GE & $\mu g / L$ \\
\hline Benzo(a)pyrene & $<10.0$ & 1 & & & WA & $\mu g / L$ \\
\hline Benzyl alcohol & $<1.00$ & 1 & & & GE & $\mu g / L$ \\
\hline Benzyl alcohol & $<10.0$ & $t$ & & & WA & $\mu g h$ \\
\hline Beryllium, total recoverable & $<0.0953$ & 1 & v & & GE & $\mu g h$ \\
\hline Beryllium, total recoverable & $<0.980$ & 1 & $\mathrm{v}$ & & WA & $\mu g / L$ \\
\hline Bis(2-chloroethoxy) methane & $<1.00$ & 1 & & & GE & $\mu g / L$ \\
\hline Bis(2-chloroethoxy) methane & $<10.0$ & 1 & & & WA & $\mu g / L$ \\
\hline Bis (2-chloroethyl) ether & $<1.00$ & 1 & & & GE & $\mu g / L$ \\
\hline Bis(2-chloroethyl) ether & $<10.0$ & 1 & & & WA & $\mu g / L$ \\
\hline Bis(2-chloroisopropyl) ether & $<20.0$ & 1 & & & GE & $\mu g / L$ \\
\hline Bis(2-chloroisopropyl) ether & $<10.0$ & 1 & & & WA & $\mu g / L$ \\
\hline Bis(2-ethylhexyl) phthalate & $<1.00$ & 1 & & & GE & $\mu g / L$ \\
\hline Bis (2-ethylhexyl) phthalate & $<3.30$ & 1 & v & & WA & $\mu g / L$ \\
\hline Bromobenzene & $<5.00$ & 1 & & & WA & $\mu \mathrm{g} / \mathrm{L}$ \\
\hline Bromochloromethane & $<5.00$ & 1 & & & WA & $\mu g / h$ \\
\hline Bromodichloromethane & $<2.00$ & 1 & & & GE & $\mu g / L$ \\
\hline Bromodichloromethane & $<5.00$ & 1 & & & WA & $\mu g h$ \\
\hline Bromoform & $<2.00$ & 1 & & & GE & $\mu g /$ \\
\hline Bromoform & $<5.00$ & 1 & & & WA & $\mu g h$ \\
\hline Bromomethane & $<2.00$ & 1 & & & GE & $\mu g / L$ \\
\hline Bromomethane & $<10.0$ & 1 & & & WA & $\mu g h$ \\
\hline 4-Bromophenyl phenyl ether & $<1.00$ & 1 & & & GE & $\mu g / L$ \\
\hline 4-Bromophenyl phenyl ether & $<10.0$ & 1 & & & WA & $\mu g / L$ \\
\hline n-Butylbenzene & $<5.00$ & 1 & & & WA & $\mu g / L$ \\
\hline sec-Butylbenzene & $<5.00$ & 1 & & & WA & $\mu \mathrm{g} / \mathrm{L}$ \\
\hline tert-Butylbenzene & $<5.00$ & 1 & & & WA & $\mu g / L$ \\
\hline Butylbenzyl phthalate & $<1.00$ & 1 & & & GE & $\mu g / L$ \\
\hline Butylbenzyl phthalate & $<10.0$ & 1 & & & WA & $\mu g /$ \\
\hline 2-sec-Butyl-4,6-dinitrophenol & $<10.0$ & 1 & & & GE & $\mu g /$ \\
\hline 2-sec-Butyl-4,6-dinitrophenol & $<50.0$ & 1 & & & WA & $\mu g / L$ \\
\hline Cadmium, total recoverable & $<2.00$ & 1 & & & GE & $\mu g / L$ \\
\hline Cadmium, total recoverable & $<4.70$ & 1 & $J X$ & & WA & $\mathrm{jgh}$ \\
\hline Carbon disulfide & $<5.00$ & 1 & & & GE & $\mu g / L$ \\
\hline Carbon disulfide & $<5.00$ & 1. & & & WA & $\mu g /$. \\
\hline Carbon tetrachloride & $<2.00$ & 1 & & & GE & $\mu g / L$ \\
\hline Carbon tetrachloride & $<5.00$ & 1 & & & WA & $\mu g / L$ \\
\hline Chlordane & $<0.123$ & 1 & & & GE & $\mu g /$ \\
\hline Chlordane & $<0.124$ & 1 & & & GE & $\mu g h$ \\
\hline alpha-Chlordane & $<0.0500$ & 1 & & & WA & $\mu g / L$ \\
\hline gamma-Chlordane & $<0.0500$ & 1 & & & WA & $\mu g h$ \\
\hline 4-Chloroaniline & $<1.00$ & 1 & & & GE & $\mu g h$ \\
\hline 4-Chloroaniline & $<10.0$ & 1 & & & WA & $\mu g / L$ \\
\hline Chlorobenzene & $<2.00$ & 1 & & & GE & $\mu g h$. \\
\hline Chlorobenzene & $<5.00$ & 1 & & & WA & $\mu \mathrm{g} / \mathrm{L}$ \\
\hline Chlorobenzilate & $<10.0$ & 1 & & & GE & $\mu g h$ \\
\hline Chlorobenzilate & $<10.0$ & 1 & & & WA & $\mu g / L$ \\
\hline 4-Chloro-m-cresol & $<10.0$ & 1 & & & WA & $\mu g / 2$ \\
\hline Chloroethane & $<2.00$ & 1 & & & GE & $\mu g / L$ \\
\hline Chloroethane & $<10.0$ & 1 & & & WA & $\mu g / L$ \\
\hline Chloroethene (Vinyl chloride) & $<2.00$ & 1 & & & GE & $\mu g / L$ \\
\hline Chloroethene (Vinyl chloride) & $<10.0$ & 1 & & & WA & $\mu g / L$ \\
\hline 2-Chloroethyl vinyl ether & $<10.0$ & 1 & & & WA & $\mu g / 2$ \\
\hline Chloroform & $<2.00$ & 1 & & & GE & $\mu g / 2$ \\
\hline Chloroform & $<5.00$ & 1 & & & WA & $\mu g / L$ \\
\hline Chloromethane & $<2.00$ & 1 & & & GE & $\mu g / L$ \\
\hline Chloromethane & $<10.0$ & 1 & & & WA & $\mu g / L$ \\
\hline 2-Chloronaphthalene & $<1.00$ & 1 & & & GE & $\mu g / L$ \\
\hline 2-Chloronaphthalene & $<10.0$ & 1 & & & WA & $\mu g / L$ \\
\hline 2-Chlorophenol & $<10.0$ & 1 & & & WA & $\mu g / L$ \\
\hline 4-Chlorophenyl phenyl ether & $<1.00$ & 1 & & & GE & $\mu g / L$ \\
\hline 4-Chlorophenyl phenyl ether & $<10.0$ & 1 & & & WA & $\mu g / L$ \\
\hline Chloroprene & $<2.00$ & 1 & & & GE & \\
\hline Chloroprene & $<5.00$ & 1 & & & WA & $\mu g / L$ \\
\hline 2-Chlorotoluene & $<5.00$ & 1 & & & WA & $\mu g / L$ \\
\hline 4-Chlorotoluene & $<5.00$ & 1 & & & WA & $\mu g / L$ \\
\hline Chromium, total recoverable & $<4.00$ & 1 & & & GE & $\mu g / L$ \\
\hline Chromium, total recoverable & $<7.00$ & 1 & & & WA & 7 \\
\hline
\end{tabular}

Note: Modifiers are listed and detailed in Appendix D, Key to Reading the Tables. $\bullet$ = exceeded holding time. 


\begin{tabular}{|c|c|c|c|c|c|c|}
\hline Parameter & $\underline{2 Q 96}$ & $\underline{\mathrm{DF}}$ & Mod & $\underline{H}$ & $\underline{\text { Lab }}$ & Unit \\
\hline Chrysene & $<0.100$ & 1 & & & GE & $\mu g / L$ \\
\hline Chrysene & $<10.0$ & 1 & & & WA & $\mu g /$ \\
\hline Cobalt, total recoverable & $<0.233$ & 1 & V & & GE & $\mu g /$ \\
\hline Cobalt, total recoverable & $<4.50$ & 1. & & & WA & $\mu g h$ \\
\hline Copper, total recoverable & 1.29 & 1 & JE & & GE & $\mu g h$ \\
\hline Copper, total recoverable & $<15.0$ & 1 & & & WA & ugh \\
\hline m/p-Cresol & $<10.0$ & 1 & & & GE & $\mu g h$ \\
\hline o-Cresol (2-Methylphenol) & $<1.00$ & 1 & & & GE & $\mu g /$ \\
\hline o-Cresol (2-Methylphenol) & $<10.0$ & 1 & & & WA & $\mu g h$ \\
\hline p-Cresol (4-Methyiphenol) & $<10.0$ & 1 & & & WA & $\mu g / 2$ \\
\hline Cyanide & $<10.0$ & 1 & & & GE & $\mu g /$ \\
\hline Cyanide & $<2.58$ & 1 & V & & WA & $\mu g / L$ \\
\hline$p, p^{\prime}-D D D$ & $<0.0490$ & 1 & & & GE & Hgh \\
\hline$p, p^{\prime}-D D D$ & $<0.0495$ & 1 & & & GE & $\mu g /$ \\
\hline$p, p^{\prime}-D D D$ & $<0.100$ & 1 & & & WA & $\mu g /$ \\
\hline$p, p^{\prime}-D D E$ & $<0.0245$ & 1 & & & GE & $\mu g / 2$ \\
\hline p,p'-DDE & $<0.0248$ & 1 & & & GE & $\mu g h$ \\
\hline$p, p^{\prime}-D D E$ & $<0.100$ & 1 & & & WA & $\mu g / L$ \\
\hline$p, p^{\prime}-D D T$ & $<0.0490$ & 1 & & & GE & $\mu g / L$ \\
\hline$p, p^{\prime}-D D T$ & $<0.0495$ & 1 & & & GE & $\mu g / L$ \\
\hline p,p'-DDT & $<0.100$ & 1 & & & WA & $\mu g /$ \\
\hline Diallate & $<10.0$ & 1 & & & GE & $\mu g / L$ \\
\hline Diallate & $<10.0$ & 1 & & & WA & $\mu g / L$ \\
\hline Dibenz(a,h)anthracene & $<0.100$ & 1 & & & GE & $\mu \mathrm{g} / \mathrm{L}$ \\
\hline Dibenz(a,h)anthracene & $<10.0$ & 1 & & & WA & $\mu g / L$ \\
\hline Dibenzofuran & $<1.00$ & 1 & & & GE & $\mu \mathrm{g} / \mathrm{L}$ \\
\hline Dibenzofuran & $<10.0$ & 1 & & & WA & $\mu g / L$ \\
\hline Dibromochloromethane & $<2.00$ & 1 & & & GE & $\mu g / L$ \\
\hline Dibromochloromethane & $<5.00$ & 1 & & & WA & $\mu g /$ \\
\hline 1,2-Dibromo-3-chloropropane & $<2.00$ & 1 & & & GE & $\mu \mathrm{g} / \mathrm{L}$ \\
\hline 1,2-Dibromo-3-chloropropane & $<10.0$ & 1 & & & WA & $\mu g / L$ \\
\hline 1,2-Dibromoethane & $<2.00$ & 1 & & & $\mathrm{GE}$ & $\mu g /$ \\
\hline 1,2-Dibromoethane & $<10.0$ & 1 & & & WA & $\mu g h$ \\
\hline Dibromomethane & $<2.00$ & 1 & & & GE & Hgh \\
\hline Dibromomethane & $<10.0$ & $i$ & & & WA & $\mu g /$ \\
\hline Di-n-butyl phthalate & $<1.00$ & 1 & & & GE & $\mu g / \mathrm{L}$ \\
\hline Di-n-butyl phthalate & $<10.0$ & 1 & & & WA & $\mu g / L$ \\
\hline 1,2-Dichlorobenzene & $<1.00$ & 1 & & & GE & $\mu g / L$ \\
\hline 1,2-Dichiorobenzene & $<5.00$ & 1 & & & WA & $\mu g / 2$ \\
\hline 1,2-Dichlorobenzene & $<10.0$ & 1 & & & WA & $\mu \mathrm{g} / \mathrm{L}$ \\
\hline 1,3-Dichlorobenzene & $<1.00$ & 1 & & & GE & $\mu g / L$ \\
\hline 1,3-Dichlorobenzene & $<5.00$ & 1 & & & WA & $\mu \mathrm{g} / \mathrm{L}$ \\
\hline 1,3-Dichiorobenzene & $<10.0$ & 1 & & & WA & $\mu g /$ \\
\hline 1,4-Dichlorobenzene & $<5.00$ & 1 & & & WA & $\mu g / 2$ \\
\hline 1,4-Dichlorobenzene & $<10.0$ & $t$ & & & WA & $\mu \mathrm{g}$ \\
\hline 3,3'-Dichlorobenzidine & $<1.00$ & 1 & & & GE & $\mu g / 2$ \\
\hline 3,3'-Dichlorobenzidine & $<20.0$ & 1 & & & WA & $\mu g / 2$ \\
\hline trans-1,4-Dichloro-2-butene & $<2.00$ & 1 & & & GE & $\mu \mathrm{g} / \mathrm{L}$ \\
\hline trans-1,4-Dichloro-2-butene & $<20.0$ & 1 & & & WA & $\mu g / L$ \\
\hline Dichlorodifluoromethane & $<2.00$ & 1 & & & GE & $\mu g / L$ \\
\hline Dichlorodifluoromethane & $<10.0$ & 1 & & & WA & rol \\
\hline 1,1-Dichloroethane & $<2.00$ & 1 & & & GE & $\mu g /$ \\
\hline 1,1-Dichloroethane & $<5.00$ & 1 & & & WA & $\mu g^{\prime}$ \\
\hline 1,2-Dichloroethane & $<2.00$ & 1 & & & GE & $\mu g /$ \\
\hline 1,2-Dichloroethane & $<5.00$ & 1 & & & WA & $\mu g / L$ \\
\hline 1,1-Dichloroethylene & $<2.00$ & 1 & & & GE & $\mu g /$ \\
\hline 1,1-Dichloroethylene & $<5.00$ & 1 & & & WA & $\mu g / 2$ \\
\hline cis-1,2-Dichloroethylene & $<5.00$ & 1 & & & WA & $\mu g h$. \\
\hline trans-1,2-Dichloroethylene & $<2.00$ & 1 & & & GE & $\mu g / \mathrm{L}$ \\
\hline trans-1,2-Dichloroethylene & $<5.00$ & 1 & & & WA & ugh \\
\hline Dichloromethane & 2.58 & 1 & JE & & GE & $\mu g / \mathrm{L}$ \\
\hline Dichloromethane & $<5.01$ & 1 & V & & WA & $\mathrm{\mu g} / \mathrm{L}$ \\
\hline 2,4-Dichlorophenol & $<1.00$ & 1 & & & GE & $\mu g /$. \\
\hline 2,4-Dichlorophenol & $<10.0$ & 1 & & & WA & $\mu g /$ \\
\hline 2,6-Dichlorophenol & $<10.0$ & 1 & & & GE & $\mu g / L$ \\
\hline 2,6-Dichlorophenol & $<10.0$ & 1 & & & WA & $\mu g / 2$ \\
\hline 2,4-Dichlorophenoxyacetic acid & $<0.0490$ & 1 & & & GE & $\mu g / L$ \\
\hline 2,4-Dichlorophenoxyacetic acid & $<1.02$ & 1 & & & WA & $\mu g / L$ \\
\hline 2,4-Dichlorophenoxyacetic acid & $<1.01$ & 1 & $J$ & & WA & jo/ \\
\hline 12-Dichloropronane & $<2.00$ & 1 & & & GE & Ha/L \\
\hline 1,2-Dichloropropane & $<5.00$ & $i$ & & & WA & Mg/L \\
\hline 1,3-Dichloropropane & $<5.00$ & 1 & & & WA & \\
\hline
\end{tabular}

Note: Modifiers are listed and detalied in Appendix D, Key to Reading the Tables.

- = exceeded holding time. 


\begin{tabular}{|c|c|c|c|c|c|c|}
\hline Parameter & $\underline{2096}$ & $\underline{\text { DF }}$ & Mod & $\underline{\mathrm{H}}$ & Lab & Unit \\
\hline 2,2-Dichloropropane & $<5.00$ & 1 & & & WA & $\mu g / 2$ \\
\hline cis-1,3-Dichloropropene & $<2.00$ & 1 & & & GE & $\mu g / L$ \\
\hline cis-1,3-Dichloropropene & $<5.00$ & 1 & & & WA & $\mu g \Omega$ \\
\hline trans-1,3-Dichloropropene & $<2.00$ & 1. & & & GE & $\mu g / 2$ \\
\hline trans-1,3-Dichloropropene & $<5.00$ & 1 & & & WA & $\mu g / \mathrm{h}$ \\
\hline Dieldrin & $<0.0245$ & 1 & & & GE & $\mu g / L$ \\
\hline Dieldrin & $<0.0248$ & 1 & & & GE & $\mu g / L$ \\
\hline Dieldrin & $<0.100$ & 1 & & & WA & $\mu g / 2$ \\
\hline Diethyl ether & $<10.0$ & 1 & & & WA & $\mu g / L$ \\
\hline Diethyl phthalate & $<1.00$ & 1 & & & GE & $\mu g / 2$ \\
\hline Diethyl phthalate & $<10.0$ & 1 & & & WA & $\mu g h$ \\
\hline Dimethoate & $<1.00$ & 1 & & & GE & $\mu g / L$ \\
\hline Dimethoate & $<0.206$ & 1 & & & WA & $\mu g / L$ \\
\hline 2,4-Dimethyl phenol & $<1.00$ & 1 & & & GE & $\mu g / \mathrm{h}$ \\
\hline 2,4-Dimethyl phenal & $<10.0$ & 1 & & & WA & $\mu g / L$ \\
\hline Dimethyl phthalate & $<1.00$ & 1 & & & GE & $\mu g / L$ \\
\hline Dimethyl phthalate & $<10.0$ & 1 & & & WA & $\mu g / L$ \\
\hline p-Dimethylaminoazobenzene & $<10.0$ & 1 & & & GE & pg/L \\
\hline p-Dimethylaminoazobenzene & $<10.0$ & 1 & & & WA & $\mu g / L$ \\
\hline 7,12-Dimethylbenz(a)anthracene & $<10.0$ & 1 & & & GE & $\mu g h$ \\
\hline 7,12-Dimethylbenz(a)anthracene & $<10.0$ & 1 & & & WA & $\mu g / L$ \\
\hline 3,3'-Dimethylbenzidine & $<10.0$ & 1 & & & GE & $\mu g / L$ \\
\hline 3,3'-Dimethylbenzidine & $<10.0$ & 1 & & & WA & $\mu g / 2$ \\
\hline a,a-Dimethylphenethylamine & $<10.0$ & 1 & & & $\mathrm{GE}$ & $\mu g / \mathrm{h}$ \\
\hline a,a-Dimethylphenethylamine & $<10.0$ & 1 & & & WA & $\mu g / L$ \\
\hline 1,3-Dinitrobenzene & $<10.0$ & 1 & & & GE & $\mu g / L$ \\
\hline 1,3-Dinitrobenzene & $<10.0$ & 1 & & & WA & $\mu g / L$ \\
\hline 2,4-Dinitrophenol & $<10.0$ & 1 & & & GE & $\mu g h$ \\
\hline 2,4-Dinitrophenol & $<50.0$ & 1 & & & WA & $\mu g /$. \\
\hline 2,4-Dinitrotaluene & $<10.0$ & 1 & & & WA & $\mu g / L$ \\
\hline 2,6-Dinitrotoluene & $<0.100$ & 1 & & & $\mathrm{GE}$ & $\mu g h$ \\
\hline 2,6-Dinitrotoluene & $<10.0$ & 1 & & & WA & $\mu g / L$ \\
\hline Di-n-octyl phthalate & $<1.00$ & 1 & & & $\mathrm{GE}$ & $\mu g / L$ \\
\hline Di-n-octyl phthalate & $<10.0$ & 1 & & & WA & $\mu g / L$ \\
\hline 1,4-Dioxane & $<10.0$ & 1 & & & GE & $\mu g / L$ \\
\hline 1,4-Dioxane & $<10.0$ & 1 & & & WA & $\mu g / L$ \\
\hline Diphenylamine & $<10.0$ & 1 & & & GE & $\mu g / L$ \\
\hline Diphenylamine & $<10.0$ & 1 & & & WA & $\mu g / L$ \\
\hline Disulfoton & $<1.00$ & 1 & & & $\mathrm{GE}$ & $\mu g / L$ \\
\hline Disulfoton & $<0.206$ & 1 & & & WA & $\mu g / L$ \\
\hline Endosulfan sulfate & $<0.0490$ & 1 & & & GE & $\mu g / L$ \\
\hline Endosulfan sulfate & $<0.0495$ & 1 & & & $\mathrm{GE}$ & $\mu g / L$ \\
\hline Endosulfan sulfate & $<0.100$ & 1 & & & WA & $\mu g / \mathrm{L}$ \\
\hline Endosulfan I & $<0.0245$ & $i$ & & & $\mathrm{GE}$ & $\mu g / L$ \\
\hline Endosulfan I & $<0.0248$ & 1 & & & $\mathrm{GE}$ & $\mu g / L$ \\
\hline Endosulfan I & $<0.0500$ & 1 & & & WA & $\mu g / L$ \\
\hline Endosulfan II & $<0.0490$ & 1 & & & GE & $\mu g / L$ \\
\hline Endosulfan II & $<0.0495$ & 1 & & & GE & $\mu g / L$ \\
\hline Endosulfan II & $<0.100$ & 1 & & & WA & $\mu g / L$ \\
\hline Endrin & $<0.0245$ & $i$ & & & $\mathrm{GE}$ & $\mu g / L$ \\
\hline Endrin & $<0.0248$ & 1 & & & GE & $\mu g / 2$ \\
\hline Endrin & $<0.100$ & $i$ & & & WA & $\mu g / L$ \\
\hline Endrin aldehyde & $<0.0613$ & 1 & & & GE & $\mu g / L$ \\
\hline Endrin aldehyde & $<0.0619$ & 1 & & & $\mathrm{GE}$ & $\mu g \Omega$ \\
\hline Endrin aldehyde & $<0.100$ & 1 & & & WA & $\mu g h$ \\
\hline Ethyl methacrylate & $<10.0$ & $i$ & & & $\mathrm{GE}$ & $\mu g h$ \\
\hline Ethyl methacrylate & $<10.0$ & 1 & & & WA & $\mu g / L$ \\
\hline Ethyl methanesulfonate & $<10.0$ & 1 & & & GE & $\mu g h$ \\
\hline Ethyl methanesulfonate & $<10.0$ & 1 & & & WA & $\mu g \Omega$ \\
\hline Ethylbenzene & $<2.00$ & 1 & & & $\mathrm{GE}$ & $\mu g /$ \\
\hline Ethylbenzene & $<5.00$ & 1 & & & WA & pgl. \\
\hline Famphur & $<10.0$ & $i$ & & & $\mathrm{GE}$ & $\mu g / 2$ \\
\hline Famphur & $<1.03$ & 1 & & & WA & $\mu g /$ \\
\hline Fluoranthene & $<1.00$ & 1 & & & GE & $\mu g \Omega$ \\
\hline Fluoranthene & $<10.0$ & 1 & & & WA & $\mu g / \mathrm{h}$ \\
\hline Fluorene & $<1.00$ & 1 & & & GE & $\mu g h$ \\
\hline Fluorene & $<10.0$ & 1 & & & WA & $\mu g h$ \\
\hline Heptachlor & $<0.0245$ & 1 & & & GE & $\mu g h$ \\
\hline Heptachlor & $<0.0248$ & 1 & & & $\mathrm{GE}$ & $\mu g h$ \\
\hline Heptachlor & $<0.0500$ & 1 & & & WA & $\mu g h$ \\
\hline Heptachlor epoxide & $<0.0245$ & 1 & & & GE & $\mu g / L$ \\
\hline Heptachlor epoxide & $<0.0248$ & 1 & & & GE & $\mu g / \mathrm{h}$ \\
\hline
\end{tabular}

Note: Modifiers are listed and detailed in Appendix D, Key to Reading the Tables.

$\bullet=$ exceeded holding time. 


\begin{tabular}{|c|c|c|c|c|c|c|}
\hline \multicolumn{7}{|c|}{ Well LFW 43C Replicate (cont.) } \\
\hline Parameter & $\underline{2096}$ & DF & $\underline{\text { Mad }}$ & $\underline{H}$ & $\underline{\text { Lab }}$ & Unit \\
\hline Heptachlor epoxide & $<0.0500$ & 1 & & & WA & $\mu g / L$ \\
\hline Heptachlorodibenzo-p-dioxins & $<0.0100$ & 1 & & & GE & $\mu g / L$ \\
\hline Heptachlorodibenzo-p-dioxins & $<0.0100$ & 1 & & & GE & $\mu g /$ \\
\hline Heptachlorodibenzo-p-dioxins & $<0.00130$ & 1. & & & WA & $\mu g \Omega$ \\
\hline Heptachiorodibenzo-p-dioxins & $<0.00200$ & 1 & & & WA & $\mu g h$ \\
\hline Heptachlorodibenzo-p-furans & $<0.0100$ & 1 & & & $\mathrm{GE}$ & $\mu g / 2$ \\
\hline Heptachlorodibenzo-p-furans & $<0.0100$ & 1 & & & $\overrightarrow{G E}$ & $\mu g / L$ \\
\hline Heptachlorodibenzo-p-furans & $<0.00250$ & 1 & & & WA & $\mu g / 2$ \\
\hline Heptachlorodibenzo-p-furans & $<0.00230$ & 1 & & & WA & $\mu g / L$ \\
\hline Hexachlorobenzene & $<0.100$ & $i$ & & & $\mathrm{GE}$ & $\mu g / L$ \\
\hline Hexachlorobenzene & $<10.0$ & 1 & & & WA & $\mu g / L$ \\
\hline Hexachlorobutadiene & $<1.00$ & $i$ & & & $\mathrm{GE}$ & $\mu g / L$ \\
\hline Hexachlorobutadiene & $<5.00$ & $i$ & & & WA & $\mu g h$ \\
\hline Hexachlorobutadiene & $<10.0$ & 1 & & & WA & $\mu g / 2$ \\
\hline Hexachlorocyclopentadiene & $<1.00$ & $i$ & & & $\mathrm{GE}$ & $\mu g / L$ \\
\hline Hexachlorocyclopentadiene & $<10.0$ & 1 & & & WA & $\mu g / \mathrm{h}$ \\
\hline Hexachlorodibenzo-p-dioxins & $<0.0100$ & $i$ & & & $\mathrm{GE}$ & $\mu g / L$ \\
\hline Hexachlorodibenzo-p-dioxins & $<0.0100$ & 1 & & & $\mathrm{GE}$ & $\mu g / L$ \\
\hline Hexachlorodibenzo-p-dioxins & $<0.00190$ & $i$ & & & WA & $\mu g / L$ \\
\hline Hexachlorodibenzo-p-dioxins & $<0.00250$ & 1 & & & WA & $\mu g / L$ \\
\hline Hexachlorodibenzo-p-furans & $<0.0100$ & 1 & & & $G E$ & $\mu g / L$ \\
\hline Hexachlorodibenzo-p-furans & $<0.0100$ & 1 & & & $\mathrm{GE}$ & $\mu g / L$. \\
\hline Hexachlorodibenzo-p-furans & $<0.00180$ & 1 & & & WA & $\mu g /$ \\
\hline Hexachlorodibenzo-p-furans & $<0.00240$ & $i$ & & & WA & $\mu g / L$ \\
\hline Hexachloroethane & $<0.100$ & 1 & & & $\mathrm{GE}$ & $\mu \mathrm{g} / \mathrm{L}$ \\
\hline Hexachloroethane & $<10.0$ & 1 & & & WA & $\mu g / L$ \\
\hline Hexachlorophene & $<80.0$ & 1 & & & $\mathrm{GE}$ & $\mu g / L$ \\
\hline Hexachlorophene & $<90.0$ & 1 & & & WA & $\mu g / L$ \\
\hline Hexachloropropene & $<10.0$ & 1 & & & GE & $\mu g h$ \\
\hline Hexachloropropene & $<10.0$ & 1 & & & WA & $\mu g h$ \\
\hline 2-Hexanone & $<10.0$ & 1 & & & GE & $\mu g / L$ \\
\hline 2-Hexanone & $<10.0$ & $i$ & & & WA & $\mu g /$ \\
\hline $1,2,3,4,6,7,8-H P C D D$ & $<0.0100$ & 1 & & & $\mathrm{GE}$ & $\mu g / L$ \\
\hline $1,2,3,4,6,7,8+\mathrm{HPCDD}$ & $<0.0100$ & $i$ & & & $G E$ & $\mu g h$ \\
\hline $1,2,3,4,6,7,8-H P C D D$ & $<0.00130$ & 1 & & & WA & $\mu g / \mathrm{L}$ \\
\hline $1,2,3,4,6,7,8-H P C D D$ & $<0.00200$ & $i$ & & & WA & $\mu g / L$ \\
\hline $1,2,3,4,6,7,8-H P C D F$ & $<0.0100$ & 1 & & & $\mathrm{GE}$ & $\mu g / 2$ \\
\hline $1,2,3,4,6,7,8-H P C D F$ & $<0.0100$ & 1 & & & $\mathrm{GE}$ & $\mu g / L$ \\
\hline $1,2,3,4,6,7,8-H P C D F$ & $<0.00230$ & 1 & & & WA & $\mu g h$ \\
\hline $1,2,3,4,6,7,8-H P C D F$ & $<0.00210$ & $i$ & & & WA & $\mu g / \mathrm{L}$ \\
\hline $1,2,3,4,7,8-H \times C D D$ & $<0.0100$ & 1 & & & $\mathrm{GE}$ & $\mu g / L$ \\
\hline $1,2,3,4,7,8-H \times C D D$ & $<0.0100$ & $i$ & & & GE & $\mu g / \mathrm{L}$ \\
\hline $1,2,3,4,7,8-H \times C D D$ & $<0.00190$ & 1 & & & WA & $\mu g / L$ \\
\hline $1,2,3,4,7,8-H \times C D D$ & $<0.00250$ & $i$ & & & WA & $\mu g / L$ \\
\hline $1,2,3,4,7,8-\mathrm{HXCDF}$ & $<0.0100$ & $i$ & & & $\mathrm{GE}$ & $\mu g / L$ \\
\hline $1,2,3,4,7,8-H \times C D F$ & $<0.0100$ & 1 & & & GE & $\mu g / L$ \\
\hline $1,2,3,4,7,8-H X C D F$ & $<0.00170$ & 1 & & & WA & $\mu g h$ \\
\hline $1,2,3,4,7,8-H \times C D F$ & $<0.00230$ & 1 & & & WA & $\mu g / L$ \\
\hline Indeno $(1,2,3-c, d)$ pyrene & $<0.100$ & 1 & & & GE & $\mu g h$ \\
\hline Indeno(1,2,3-c,d)pyrene & $<10.0$ & $i$ & & & WA & $\mu g / L$ \\
\hline lodomethane (Methyl iodide) & $<10.0$ & $i$ & & & $\mathrm{GE}$ & $\mu g / L$ \\
\hline lodomethane (Methyl iodide) & $<5.00$ & 1 & & & WA & $\mu g / L$ \\
\hline Isobutyl alcohol & $<20.0$ & $i$ & & & GE & $\mu g / L$ \\
\hline Isobutyl alcohol & $<50.0$ & 1 & & & WA & $\mu g / L$ \\
\hline Isodrin & $<10.0$ & $i$ & & & GE & $\mu g /$ \\
\hline Isodrin & $<0.100$ & $i$ & & & WA & $\mu g /$ \\
\hline Isophorone & $<1.00$ & $i$ & & & $\mathrm{GE}$ & $\mu g / \mathrm{L}$ \\
\hline Isophorone & $<10.0$ & 1 & & & WA & $\mu g h$ \\
\hline Isopropylbenzene & $<5.00$ & $i$ & & & WA & $\mu g \mathrm{~L}$ \\
\hline p-lsopropyltoluene & $<5.00$ & 1 & & & WA & $\mu g / L$ \\
\hline $\begin{array}{l}\text { Isosairole } \\
\text { Intel }\end{array}$ & $<10.0$ & 1 & & & $\mathrm{GE}$ & $\mu g h$ \\
\hline Isosafrole & $<10.0$ & 1 & & & WA & $\mu g / L$ \\
\hline Kepone & $<10.0$ & $i$ & & & $G E$ & $\mu g / L$ \\
\hline Kepone & $<0.500$ & $i$ & & & WA & $\mu g h$ \\
\hline Lead, total recoverable & $<5.00$ & $i$ & & & $\mathrm{GE}$ & $\mu g / \mathrm{L}$ \\
\hline Lead, total recoverable & $<47.0$ & 1 & & & WA & $\mu g h$ \\
\hline Lindane & $<0.0123$ & $i$ & & & $\mathrm{GE}$ & $\mu g / L$. \\
\hline Lindane & $<0.0124$ & 1 & & & GE & $\mu g / L$ \\
\hline Lindane & $<0.0500$ & $i$ & & & WA & $\mu g / L$ \\
\hline Mercury, total recoverable & $<0.200$ & 1 & & & $\mathrm{GE}$ & $\mu g / L$ \\
\hline Mercury, total recoverable & $<0.200$ & 1 & & & GE & $\mu g / 2$ \\
\hline Mercury, total recoverable & $<0.700$ & $i$ & & & WA & $\mu g / L$ \\
\hline
\end{tabular}

Note: Modifiers are listed and detailed in Appendix D, Key to Reading the Tables. - = exceeded holding time. 


\begin{tabular}{|c|c|c|c|c|c|c|}
\hline Parameter & $\underline{2096}$ & DF & Mod & $\underline{H}$ & Lab & Unit \\
\hline Methacrylonitrile & $<10.0$ & 1 & & & GE & $\mu g / L$ \\
\hline Methacrylonitrile & $<20.0$ & 1 & & & WA & $\mu \mathrm{g} / \mathrm{L}$ \\
\hline Methapyrilene & $<10.0$ & 1 & & & GE & $\mu g \Omega$ \\
\hline Methapyrilene & $<10.0$ & 1. & & & WA & $\mu g / L$ \\
\hline Methoxychlor & $<0.245$ & 1 & & & GE & $\mu g / L$ \\
\hline Methoxychlor & $<0.248$ & 1 & & & GE & $\mu \mathrm{g} / \mathrm{L}$ \\
\hline Methoxychlor & $<0.500$ & 1 & & & WA & $\mu g / L$ \\
\hline 2-Methyl-4,6-dinitrophenol & $<10.0$ & 1 & & & GE & $\mu g / L$ \\
\hline 2-Methyl-4,6-dinitrophenol & $<50.0$ & 1 & & & WA & $\mu g / L$ \\
\hline Methyl ethyl ketone & $<5.00$ & 1 & & & GE & $\mu \mathrm{g} / \mathrm{L}$ \\
\hline Methyl ethyl ketone & $<10.0$ & 1 & & & WA & $\mu g / L$ \\
\hline Methyl isobutyl ketone & $<10.0$ & 1 & & & $\mathrm{GE}$ & $\mu g h$ \\
\hline Methyl isobutyl ketone & $<10.0$ & 1 & & & WA & $\mu g / L$ \\
\hline Methyl methacrylate & $<10.0$ & 1 & & & GE & $\mu g / L$ \\
\hline Methyl methacrylate & $<10.0$ & 1 & & & WA & $\mu g /$ \\
\hline Methyl methanesulfonate & $<10.0$ & 1 & & & GE & pghl \\
\hline Methyl methanesulfonate & $<10.0$ & 1 & & & WA & $\mu g / L$ \\
\hline 3-Methylcholanthrene & $<10.0$ & 1 & & & GE & $\mu g / L$ \\
\hline 3-Methylcholanthrene & $<10.0$ & 1 & & & WA & $\mu g / L$ \\
\hline 2-Methylnaphthalene & $<1.00$ & 1 & & & GE & $\mu g / L$ \\
\hline 2-Methylnaphthalene & $<10.0$ & 1 & & & WA & $\mu g / L$ \\
\hline Naphthalene & $<1.00$ & 1 & & & GE & $\mu g / L$ \\
\hline Naphthalene & $<5.00$ & 1 & & & WA & $\mu g / L$ \\
\hline Naphthalene & $<10.0$ & 1 & & & WA & $\mu g / L$. \\
\hline 1,4-Naphthoquinone & $<10.0$ & 1 & & & GE & $\mu g / L$ \\
\hline 1,4-Naphthoquinone & $<10.0$ & 1 & & & WA & $\mu g / L$ \\
\hline 1-Naphthylamine & $<10.0$ & 1 & & & GE & $\mu g / L$ \\
\hline 1-Naphthylamine & $<10.0$ & 1 & & & WA & $\mu g / L$ \\
\hline 2-Naphthylamine & $<10.0$ & 1 & & & GE & $\mu g / L$ \\
\hline 2-Naphthylamine & $<10.0$ & 1 & & & WA & $\mu g / L$ \\
\hline Nickel, total recoverable & $<10.0$ & 1 & & & GE & $\mu g / L$ \\
\hline Nickel, total recoverable & $<26.0$ & 1 & & & WA & $\mu g / 2$ \\
\hline m-Nitroaniline & $<1.00$ & 1 & & & GE & $\mu g / L$ \\
\hline m-Nitroaniline & $<50.0$ & 1 & & & WA & $\mu g / 2$ \\
\hline o-Nitroaniline & $<1.00$ & 1 & & & GE & $\mu g / L$ \\
\hline o-Nitroaniline & $<50.0$ & 1 & & & WA & jg/L \\
\hline p-Nitroaniline & $<1.00$ & 1 & & & GE & $\mu g / L$ \\
\hline p-Nitroaniline & $<50.0$ & 1 & & & WA & $\mu g / L$ \\
\hline Nitrobenzene & $<1.00$ & 1 & & & GE & $\mu g / L$ \\
\hline Nitrobenzene & $<10.0$ & 1 & & & WA & $\mu g / L$ \\
\hline 2-Nitrophenol & $<1.00$ & 1 & & & GE & $\mu g /$ \\
\hline 2-Nitrophenol & $<10.0$ & 1 & & & WA & $\mu g / L$ \\
\hline 4-Nitrophenol & $<1.00$ & 1 & J & & GE & $\mu g h$ \\
\hline 4-Nitrophenol & $<50.0$ & 1 & & & WA & $\mu g / 2$ \\
\hline 4-Nitroquinoline-1-oxide & $<10.0$ & 1 & & & GE & $\mu g / L$ \\
\hline 4-Nitroquinoline-1-oxide & $<20.0$ & 1 & & & WA & $\mu g / L$ \\
\hline N-Nitrosodi-n-butylamine & $<10.0$ & 1 & & & GE & $\mu g h$ \\
\hline N-Nitrosodi-n-butylamine & $<10.0$ & 1 & & & WA & $\mu g / L$ \\
\hline N-Nitrosodiethylamine & $<10.0$ & 1 & & & GE & $\mu g / L$ \\
\hline N-Nitrosodiethylamine & $<10.0$ & 1 & & & WA & $\mu g / L$ \\
\hline N-Nitrosodimethylamine & $<1.00$ & 1 & & & $\mathrm{GE}$ & $\mu g / L$ \\
\hline N-Nitrosodimethylamine & $<10.0$ & 1 & & & WA & $\mu g / L$ \\
\hline N-Nitrosodiphenylamine & $<1.00$ & 1 & & & $\mathrm{GE}$ & $\mu g / L$ \\
\hline N-Nitrosodiphenylamine & $<10.0$ & 1 & & & WA & $\mu g / L$ \\
\hline N-Nitrosodipropylamine & $<10.0$ & 1 & & & WA & $\mu g / L$ \\
\hline N-Nitrosomethylethylamine & $<10.0$ & 1 & & & $\mathrm{GE}$ & $\mu g / L$ \\
\hline N-Nitrosomethylethylamine & $<10.0$ & 1 & & & WA & $\mu g / L$ \\
\hline N-Nitrosomorpholine & $<10.0$ & 1 & & & GE & $\mu g / L$ \\
\hline N-Nitrosomorpholine & $<10.0$ & 1 & & & WA & $\mu g / L$ \\
\hline N-Nitrosopiperidine & $<10.0$ & 1 & & & GE & $\mu g / L$ \\
\hline N-Nitrosopiperidine & $<50.0$ & 1 & & & WA & $\mu g / L$ \\
\hline N-Nitrosopyrrolidine & $<10.0$ & 1 & & & GE & $\mu g / L$ \\
\hline N-Nitrosopyrrolidine & $<10.0$ & 1 & & & WA & $\mu g / L$ \\
\hline 5-Nitro-o-toluidine & $<10.0$ & 1 & & & GE & $\mu g / L$ \\
\hline 5-Nitro-0-toluidine & $<10.0$ & 1 & & & WA & $\mu g / L$ \\
\hline Octachlorodibenzo-p-dioxin & $<0.0100$ & 1 & & & GE & $\mu \mathrm{g} / \mathrm{L}$ \\
\hline Octachlorodibenzo-p-dioxin & $<0.0100$ & 1 & & & $\mathrm{GE}$ & $\mu g / L$ \\
\hline Octachlorodibenzo-p-dioxin & $<0.00240$ & 1 & & & WA & $\mu g / L$ \\
\hline Octachlorodibenzo-p-dioxin & $<0.00300$ & 1 & & & WA & $\mu g / L$ \\
\hline Octachlorodibenzo-p-furan & $<0.0100$ & 1 & & & GE & $\mu g / \mathrm{L}$ \\
\hline Octachlorodibenzo-p-furan & $<0.0100$ & 1 & & & GE & $\mu g / L$ \\
\hline Octachlorodibenzo-p-furan & $<0.00220$ & 1 & & & WA & $\mu g / L$ \\
\hline Octachlorodibenzo-o-furan & $<0.00210$ & 1 & & & WA & \\
\hline
\end{tabular}

Note: Modifiers are listed and detailed in Appendix D, Key to Reading the Tables.

$\bullet=$ exceeded holding time. 


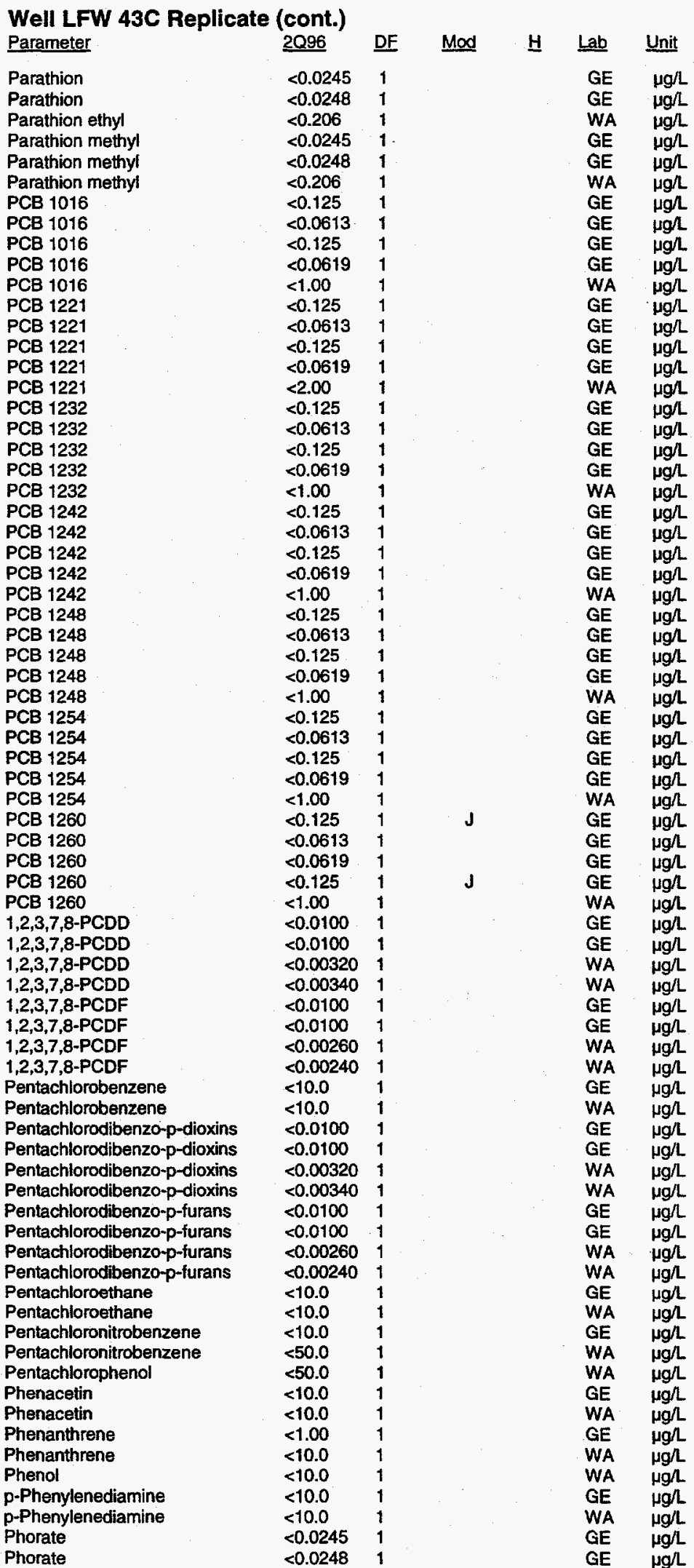

Note: Modifiers are listed and detailed in Appendix D, Key to Reading the Tables. - $=$ exceeded holding time. 


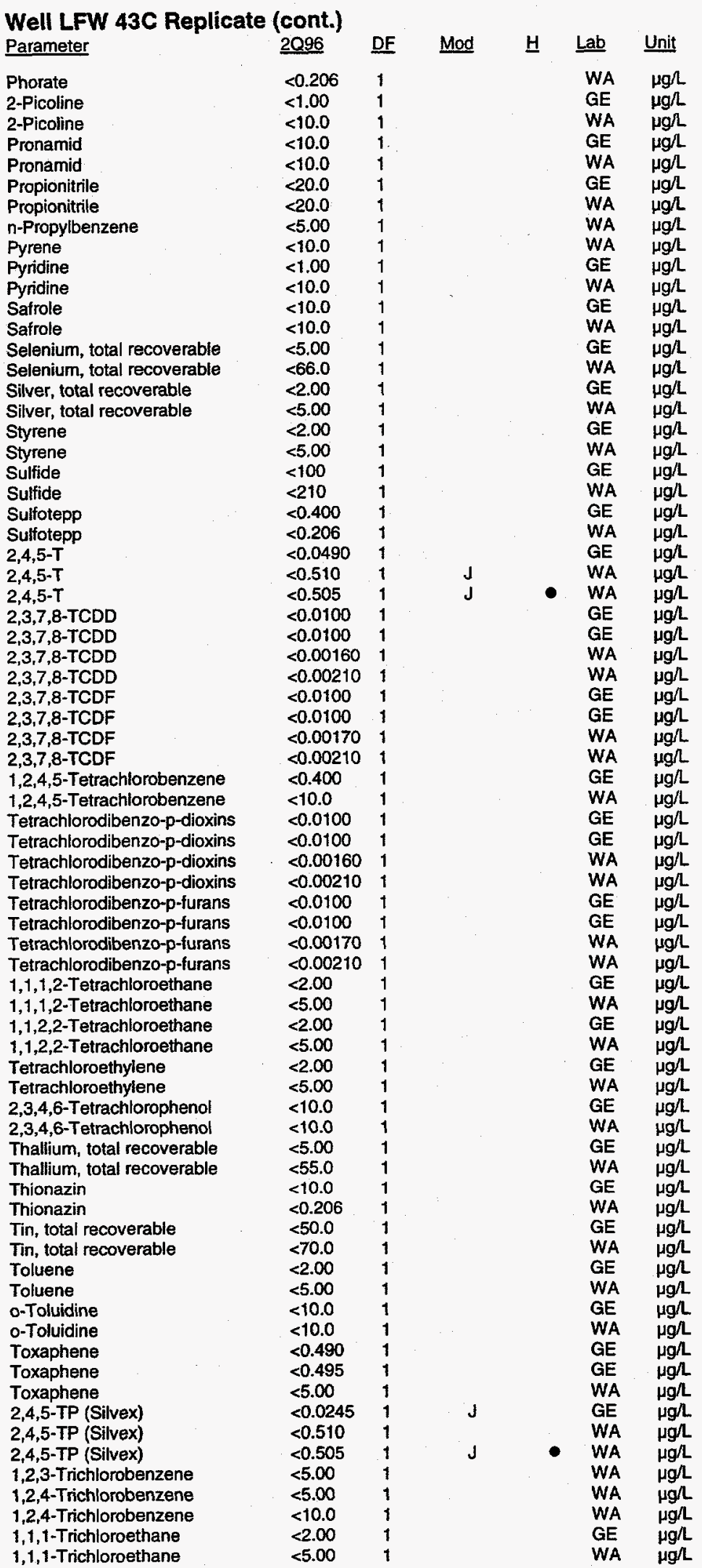

Note: Modifiers are listed and detailed in Appendix D, Key to Reading the Tables. - = exceeded holding time. 
Well LFW 43C Replicate (cont.)

Parameter

1,1,2-Trichloroethane

Trichloroethylene

Trichloroethylene

Trichlorofluoromethane

Trichlorofluoromethane

2,4,5-Trichlorophenol

2,4,5-Trichloropheno

2,4,6-Trichloropheno

2,4,6-Trichlorophenol

1,2,3-Trichloropropane

1,2.3-Trichloropropane

Trichlorotrifluoroethane

$0,0,0$-Triethyl phosphorothioate

$0,0,0-T r i e t h y l$ phosphorothioate

1,2,4-Trimethylbenzene

1,3,5-Trimethylbenzene

1,3,5-Trinitrobenzene

1,3,5-Trinitrobenzene

Vanadium, total recoverable

Vanadium, total recoverable

Vinyl acetate

Vinyl acetate

$\mathrm{m} / \mathrm{p}$-Xylene

o-Xylene

Xylenes

Zinc, total recoverable

Zinc, total recoverable $\underline{2096}$

$<2.00$

$<2.00$

$<5.00$

$<2.00$

$<5.00$

$<1.00$

$<50.0$

$<0.100$

$<10.0$

$<2.00$

$<5.00$

$<5.00$

$<10.0$

$<0.206$

$<5.00$

$<5.00$

$<10.0$

$<10.0$

$<10.0$

$<6.90$

$<10.0$

$<10.0$

$<5.00$

$<5.00$

$<2.00$

4.63

$<12.3$
DF $\quad$ Mod

H Lab Unit

GE $\quad \mu g / L$

GE $\quad \mu g / L$

GE $\quad \mu g / L$

WA $\mu g / L$

GE $\mu g / L$

WA $\mu g / L$

GE $\quad \mu g /$

WA $\mu g h$

WA $\mu g / L$

WA $\mu g \Omega$

GE $\mu g / L$

WA $\mu g / L$

WA $\mu g / L$

WA $\mu g /$ L

$\mu g$ L

WA $\quad \mu g / L$

GE $\quad \mu g / L$

GE

WA $\mu g / L$

WA $\mu g / L$

WA $\mu g / L$

GE $\quad \mu g / L$

JE GE $\quad \mu g / L$

Note: Modifiers are listed and detailed in Appendix D, Key to Reading the Tables.

$\bullet=$ exceeded holding time. 
WELL LFW 43D

SAS Coord.

N86443.2

E45244.5

SAMPLE DATE

Latlongitude

$33.290770^{\circ} \mathrm{N}$ $81.715580^{\circ} \mathrm{W}$

$04 / 22 / 96$

FIELD DATA

Parameter

Water elevation

pH

Sp. conductance

Water temperature

Alkalinity as $\mathrm{CaCO}_{3}$

Turbidity

Volume purged

Sampling code

ANALYTICAL DATA

Parameter

Acenaphthylene

Acetone

Acetonitrile (Methyl cyanide)

Acetophenone

2-Acetylaminofluorene

Acrolein

Acrylonitrile

Aldrin

Allyl chloride

4-Aminobiphenyl

Aniline

Anthracene

Antimony, total recoverable

Aramite

Arsenic, total recoverable

Barium, total recoverable

Benzene

alpha-Benzene hexachloride

beta-Benzene hexachloride

delta-Benzene hexachloride

Benzo(a)anthracene

Benzo(b)fluoranthene

Benzo(k)fluoranthene

Benzo(g,h,i)perylene

Benzo(a)pyrene

Benzyl alcohol

Beryllium, total recoverable

Bis(2-chloroethoxy) methane

Bis(2-chloroethyl) ether

Bis(2-chloroisopropyl) ether

Bis(2-ethylhexyl) phthalate

Bromodichloromethane

Bromoform

Bromomethane

4-Bromophenyl phenyl ether

Butylbenzyl phthaiate

2-sec-Butyl-4,6-dinitrophenol

Cadmium, total recoverable

Carbon disulfide

Carbon tetrachloride

Chlordane

4-Chloroaniline

Chlorobenzene

Chlorobenzilate

Chloroethane

Chloroethene (Vinyl chloride)

Chloroform

Chloromethane

2-Chloronaphthalene

4-Chlorophenyl phenyl ether

Chloroprene $\underline{2096}$

166.1

5

12
25.0

5.9
Screen Zone Elevation

$170.9-150.9 \mathrm{ft} \mathrm{ms}$

Top of Casing

Casing

Pump

$202.9 \mathrm{ft} \mathrm{msl}$

4" PVC
Screen Zone

Upper Steed Pond
Unit

ft msl

$\mathrm{pH}$

$\mu \mathrm{S} / \mathrm{cm}$

${ }^{\circ} \mathrm{C}$

mgl

well vol

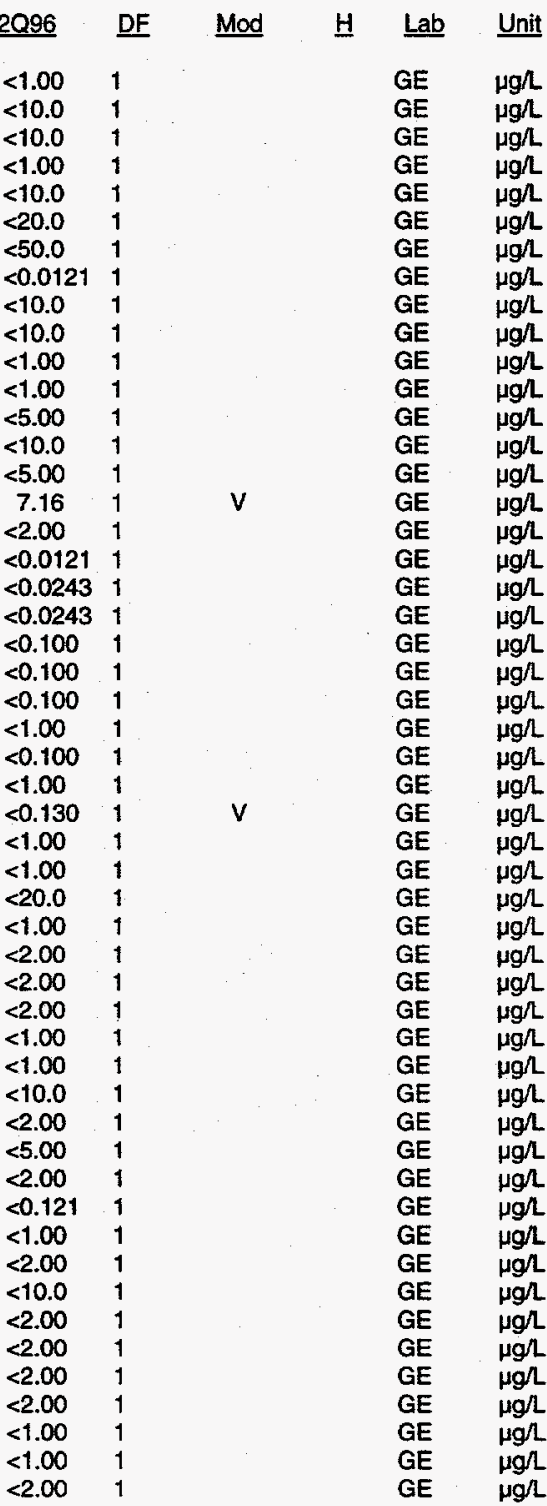

Note: Modifiers are listed and detailed in Appendix D, Key to Reading the Tables.

$\bullet=$ exceeded holding time. 
Well LFW 43D (cont.)

\section{Parameter}

Chromium, total recoverable

Chrysene

Cobalt, total recoverable

Copper, total recoverable $\mathrm{m} / \mathrm{p}$-Cresol

o-Cresol (2-Methylphenol)

Cyanide

$p, p^{\prime}-D D D$

$p, p^{\prime}-D D E$

p,p'-DDT

Diallate

Dibenz(a,h)anthracene

Dibenzofuran

Dibromochloromethane

1,2-Dibromo-3-chloropropane

1,2-Dibromoethane

Dibromomethane

Di-n-butyl phthaiate

1,2-Dichlorobenzene

1,3-Dichlorobenzene

3,3'-Dichlorobenzidine

trans-1,4-Dichloro-2-butene

Dichlorodifluoromethane

1,1-Dichloroethane

1,2-Dichloroethane

1,1-Dichloroethylene

trans-1,2-Dichloroethylene

Dichloromethane

2,4-Dichlorophenol

2,6-Dichlorophenol

2,4-Dichlorophenoxyacetic acid

1,2-Dichloropropane

cis-1,3-Dichloropropene

trans-1,3-Dichloropropene

Dieldrin

Diethyl phthalate

Dimethoate

2,4-Dimethyl phenol

Dimethyl phthalate

p-Dimethylaminoazobenzene

7,12-Dimethylbenz(a)anthracene

3,3'-Dimethylbenzidine

a.a-Dimethylphenethylamine

1,3-Dinitrobenzene

2,4-Dinitrophenol

2,6-Dinitrotoluene

Di-n-octyl phthalate

1,4-Dioxane

Diphenylamine

Disulfoton

Endosulfan sulfate

Endosulfan I

Endosulfan II

Endrin

Endrin aldehyde

Ethyl methacrylate

Ethyl methanesulfonate

Ethylbenzene

Famphur

Fluoranthene

Fluorene

Heptachlor

Heptachlor epoxide

Heptachlorodibenzo-p-dioxins

Heptachlorodibenzo-p-furans

Hexachlorobenzene

Hexachlorobutadiene

Hexachlorocyclopentadien

Hexachlorodibenzo-p-dioxins

Hexachlorodibenzo-p-furans

Hexachloroethane

Hexachlorophene

\begin{tabular}{|c|c|c|c|c|c|}
\hline 2096 & DF & Mod & H & Lab & Unit \\
\hline$<4.00$ & 1 & & & GE & $\mu g /$ \\
\hline$<0.100$ & 1 & & & GE & $\mu g / L$ \\
\hline$<0.297$ & 1 & $V$ & & GE & $\mu g / 2$ \\
\hline 2.99 & 1 & JE & & $\mathrm{GE}$ & $\mu g / L$ \\
\hline$<10.0$ & 1 & & & GE & $\mu g / L$ \\
\hline$<1.00$ & 1 & & & GE & $\mu g / L$ \\
\hline$<10.0$ & 1 & & & GE & $\mu g / L$ \\
\hline$<0.0485$ & 1 & & & GE & $\mu g / L$ \\
\hline$<0.0243$ & 1 & & & GE & $\mu g / L$ \\
\hline$<0.0485$ & 1 & & & GE & $\mu g / L$ \\
\hline$<10.0$ & 1 & & & GE & $\mu g / L$ \\
\hline$<0.100$ & 1 & & & GE & $\mu g / L$ \\
\hline$<1.00$ & 1 & & & GE & $\mu g /$ \\
\hline$<2.00$ & 1 & & & GE & $\mu g / L$ \\
\hline$<2.00$ & 1 & & & GE & $\mu g /$ \\
\hline$<2.00$ & 1 & & & GE & $\mu g / L$ \\
\hline$<2.00$ & 1 & & & GE & $\mu g / L$ \\
\hline$<1.00$ & 1 & & & GE & $\mu g \Omega$ \\
\hline$<1.00$ & 1 & & & GE & $\mu g /$. \\
\hline$<1.00$ & 1 & & & GE & $\mu g /$ \\
\hline$<1.00$ & 1 & & & GE & $\mu g /$ \\
\hline$<2.00$ & 1 & & & $\mathrm{GE}$ & $\mu g / L$ \\
\hline$<2.00$ & 1 & & & GE & $\mu g / L$ \\
\hline$<2.00$ & 1 & & & GE & $\mu g / L$ \\
\hline$<2.00$ & 1 & & & GE & $\mu g / L$ \\
\hline$<2.00$ & 1 & & & GE & $\mu g / L$ \\
\hline$<2.00$ & 1 & & & GE & \\
\hline 3.19 & 1 & JE & & GE & $\mu g / L$ \\
\hline$<1.00$ & 1 & & & GE & $\mu g / L$ \\
\hline$<10.0$ & 1 & & & GE & $\mu g /$ \\
\hline$<0.0490$ & 1 & & & GE & $\mu g / L$ \\
\hline$<2.00$ & 1 & & & GE & $\mu g / L$ \\
\hline$<2.00$ & 1 & & & GE & $\mu g / L$ \\
\hline$<2.00$ & 1 & & & GE & $\mu g / L$ \\
\hline$<0.0243$ & 1 & & & GE & $\mu g /$ \\
\hline$<1.00$ & 1 & & & GE & $\mu g / L$ \\
\hline$<1.00$ & 1 & & & GE & \\
\hline$<1.00$ & 1 & & & GE & $\mu g /$ \\
\hline$<1.00$ & 1 & & & GE & $\mu g /$ \\
\hline$<10.0$ & 1 & & & GE & $\mu g / L$ \\
\hline$<10.0$ & 1 & & & GE & $\mu g /$ \\
\hline$<10.0$ & 1 & & & GE & $\mu g /$ \\
\hline$<10.0$ & 1 & & & GE & jg/ \\
\hline$<10.0$ & 1 & & & GE & \\
\hline$<10.0$ & 1 & & & GE & $\mu g$ \\
\hline$<0.100$ & 1 & & & GE & \\
\hline$<1.00$ & 1 & & & GE & $\mu g$ \\
\hline$<10.0$ & 1 & & & GE & $\mu \mathrm{g}$ \\
\hline$<10.0$ & 1 & & & GE & $\mu g / L$ \\
\hline$<1.00$ & 1 & & & GE & \\
\hline$<0.0485$ & 1 & & & GE & $\mu g / L$ \\
\hline$<0.0243$ & 1 & & & GE & \\
\hline$<0.0485$ & 1 & & & GE & \\
\hline$<0.0243$ & 1 & & & GE & \\
\hline$<0.0607$ & 1 & & & GE & \\
\hline$<10.0$ & 1 & & & GE & $\mu g$ \\
\hline$<10.0$ & 1 & & & GE & $\mu g$ \\
\hline$<2.00$ & 1 & & & GE & \\
\hline$<10.0$ & 1 & & & GE & \\
\hline$<1.00$ & 1 & & & GE & \\
\hline$<1.00$ & 1 & & & GE & \\
\hline$<0.0243$ & 1 & & & GE & \\
\hline$<0.0243$ & 1 & & & GE & \\
\hline$<0.0100$ & 1 & & & GE & \\
\hline$<0.0100$ & 1 & & & GE & \\
\hline$<0.100$ & 1 & & & GE & \\
\hline$<1.00$ & 1 & & & GE & \\
\hline$<1.00$ & 1 & & & GE & \\
\hline$<0.0100$ & 1 & & & GE & \\
\hline$<0.0100$ & 1 & & & GE & \\
\hline$<0.100$ & 1 & & & GE & \\
\hline$<80.0$ & 1 & & & GE & \\
\hline
\end{tabular}

Note: Modifiers are listed and detailed in Appendix D, Key to Reading the Tables.

$-=$ exceeded holding time. 
Well LFW 43D (cont.)

\section{Parameter}

Hexachloropropene

2-Hexanone

1,2,3,4,6,7,8-HPCDD

$1,2,3,4,6,7,8-H P C D F$

$1,2,3,4,7,8-H X C D D$

$1,2,3,4,7,8-\mathrm{HXCDF}$

indeno(1,2,3-c,d)pyrene

lodomethane (Methyl iodide)

Isobutyl alcohol

Isodrin

Isophorone

Isosafrole

Kepone

Lead, total recoverable

Lindane

Mercury, total recoverable

Methacrylonitrile

Methapyrilene

Methoxychlor

2-Methyl-4,6-dinitrophenol

Methyl ethyl ketone

Methyl isobutyl ketone

Methyl methacrylate

Methyl methanesulfonate

3-Methyicholanthrene

2-Methyinaphthalene

Naphthalene

1,4-Naphthoquinone

1-Naphthylamine

2-Naphthylamine

Nickel, total recoverable

m-Nitroaniline

o-Nitroaniline

p-Nitroaniline

Nitrobenzene

2-Nitrophenol

4-Nitrophenol

4-Nitroquinoline-1-oxide

N-Nitrosodi-n-butylamine

$\mathrm{N}$-Nitrosodiethylamine

N-Nitrosodimethylamine

$\mathrm{N}$-Nitrosodiphenylamine

$\mathrm{N}$-Nitrosomethylethylamine

$\mathrm{N}$-Nitrosomorpholine

$N$-Nitrosopiperidine

N-Nitrosopyrrolidine

5-Nitro-o-toluidine

Octachlorodibenzo-p-dioxin

Octachlorodibenzo-p-furan

Parathion

Parathion methyl

PCB 1016

PCB 1016

PCB 1221

PCB 1221

PCB 1232

PCB 1232

PCB 1242

PCB 1242

PCB 1248

PCB 1248

PCB 1254

PCB 1254

PCB 1260

PCB 1260

1,2,3,7,8-PCDD

1,2,3,7,8-PCDF

Pentachlorobenzene

Pentachlorodibenzo-p-dioxins

Pentachlorodibenzo-p-furans

Pentachloroethane

Pentachloronitrobenzene

Phenacetin

\begin{tabular}{|c|c|c|c|c|c|}
\hline$\underline{2096}$ & DF & Mod & $\underline{\mathrm{H}}$ & Lab & $\underline{\text { Unit }}$ \\
\hline$<10.0$ & 1 & & & $G E$ & $\mu g / L$ \\
\hline$<10.0$ & 1 & & & $\overline{G E}$ & $\mu g h$ \\
\hline$<0.0100$ & 1 & & & GE & $\mu g /$ \\
\hline$<0.0100$ & 1 & & & GE & $\mu g h$ \\
\hline$<0.0100$ & 1 & & & GE & $\mu g /$ \\
\hline$<0.0100$ & $i$ & & & GE & $\mu g /$ \\
\hline$<0.100$ & 1 & & & GE & $\mu g h$ \\
\hline$<10.0$ & $i$ & & & GE & $\mu g /$ \\
\hline$<20.0$ & 1 & & & GE & $\mu g h$ \\
\hline$<10.0$ & 1 & & & GE & $\mu g / L$ \\
\hline$<1.00$ & 1 & & & $\overline{G E}$ & $\mu g /$. \\
\hline$<10.0$ & 1 & & & GE & $\mu g / \mathrm{L}$ \\
\hline$<10.0$ & 1 & & & GE & $\mu g / \mathrm{L}$ \\
\hline$<5.00$ & 1 & & & $\overrightarrow{\mathrm{GE}}$ & $\mu g /$ \\
\hline$<0.0121$ & 1 & & & GE & $\mu g / L$ \\
\hline$<0.200$ & 1 & & & $\mathrm{GE}$ & $\mu g /$ \\
\hline$<10.0$ & 1 & & & $\mathrm{GE}$ & $\mu g / L$ \\
\hline$<10.0$ & 1 & & & $\overline{G E}$ & $\mu g / L$ \\
\hline$<0.243$ & 1 & & & GE & $\mu g /$ \\
\hline$<10.0$ & 1 & & & GE & $\mathrm{pg} / \mathrm{L}$ \\
\hline$<5.00$ & 1 & & & GE & $\mu g / 2$ \\
\hline$<10.0$ & 1 & & & $\mathrm{GE}$ & $\mu g /$ \\
\hline$<10.0$ & 1 & & & $\mathrm{GE}$ & $\mu g h$ \\
\hline$<10.0$ & 1 & & & GE & $\mathrm{Hg} / \mathrm{L}$ \\
\hline$<10.0$ & 1 & & & $\overline{\mathrm{GE}}$ & $\mu g^{\prime}$ \\
\hline$<1.00$ & 1 & & & GE & $\mu g / \mathrm{L}$ \\
\hline$<1.00$ & 1 & & & $\overline{G E}$ & $\mu g / L$ \\
\hline$<10.0$ & 1 & & & $\overrightarrow{G E}$ & $\mu g /$ \\
\hline$<10.0$ & 1 & & & $\mathrm{GE}$ & $\mu g / L$ \\
\hline$<10.0$ & 1 & & & GE & $\mu g /$ \\
\hline$<10.0$ & 1 & & & $\overline{G E}$ & $\mu \mathrm{g} / \mathrm{L}$ \\
\hline$<1.00$ & 1 & & & $\mathrm{GE}$ & $\mu g / L$ \\
\hline$<1.00$ & 1 & & & $\overline{G E}$ & ugl \\
\hline$<1.00$ & 1 & & & $\mathrm{GE}$ & $\mu \mathrm{g} / \mathrm{L}$ \\
\hline$<1.00$ & 1 & & & GE & $\mu g /$ \\
\hline$<1.00$ & 1 & & & $\mathrm{GE}$ & $\mu g /$ \\
\hline$<1.00$ & 1 & $J$ & & GE & $\mu g /$ \\
\hline$<10.0$ & 1 & & & GE & pl \\
\hline$<10.0$ & 1 & & & $\mathrm{GE}$ & pgl \\
\hline$<10.0$ & 1 & & & GE & $\mu g /$ \\
\hline$<1.00$ & 1 & & & $\overline{G E}$ & $\mu \mathrm{g}$ \\
\hline$<1.00$ & 1 & & & $\mathrm{GE}$ & ug/l \\
\hline$<10.0$ & 1 & & & GE & $\mu g /$ \\
\hline$<10.0$ & 1 & & & GE & $\mu / \operatorname{lo}$ \\
\hline$<10.0$ & 1 & & & $\mathrm{GE}$ & pol \\
\hline$<10.0$ & 1 & & & $\mathrm{GE}$ & $\mu g^{\prime}$ \\
\hline$<10.0$ & 1 & & & $\mathrm{GE}$ & $\mu g / L$ \\
\hline$<0.0100$ & 1 & & & GE & $\mu g^{\prime} L$ \\
\hline$<0.0100$ & 1 & & & $\overline{G E}$ & $\mu g / \mathrm{L}$ \\
\hline$<0.0243$ & $i$ & & & GE & $\mu g$ \\
\hline$<0.0243$ & 1 & & & GE & ya/L \\
\hline$<0.125$ & 1 & & & $\mathrm{GE}$ & $\mu g / \mathrm{L}$ \\
\hline$<0.0607$ & $i$ & & & $\mathrm{GE}$ & Ho/l \\
\hline$<0.125$ & $i$ & & & GE & $\mu g /$ \\
\hline$<0.0607$ & 1 & & & GE & $\mu g /$ \\
\hline$<0.125$ & 1 & & & GE & $\mu g /$ \\
\hline$<0.0607$ & 1 & & & $\mathrm{GE}$ & $\mu$ \\
\hline$<0.125$ & 1 & & & $\mathrm{GE}$ & ug \\
\hline$<0.0607$ & 1 & & & GE & \\
\hline$<0.125$ & $i$ & & & $\mathrm{GE}$ & $\mu g^{\prime}$ \\
\hline$<0.0607$ & 1 & & & GE & ual \\
\hline$<0.125$ & 1 & & & GE & $\mu \mathrm{g} / \mathrm{l}$ \\
\hline$<0.0607$ & 1 & & & $\mathrm{GE}$ & $\mu g$ \\
\hline$<0.0607$ & 1 & & & GE & $\mu g /$ \\
\hline$<0.125$ & 1 & $J$ & & GE & $\mu \mathrm{g} / \mathrm{h}$ \\
\hline$<0.0100$ & 1 & & & GE & $\mu g /$ \\
\hline$<0.0100$ & 1 & & & GE & $\mu g /$ \\
\hline$<10.0$ & 1 & & & $\mathrm{GE}$ & igh \\
\hline$<0.0100$ & 1 & & & GE & yal \\
\hline$<0.0100$ & 1 & & & $\mathrm{GE}$ & $\mu g / L$ \\
\hline$<10.0$ & 1 & & & GE & \\
\hline$<10.0$ & 1 & & & GE & \\
\hline$<10.0$ & 1 & & & GE & \\
\hline
\end{tabular}

Note: Modifiers are listed and detailed in Appendix D, Key to Reading the Tables.

- = exceeded holding time. 
Well LFW 43D (cont.)

Parameter

Phenanthrene

p-Phenylenediamine

Phorate

2-Picoline

Pronamid

Propionitrile

Pyridine

Safrole

Selenium, total recoverable

Silver, total recoverable

Styrene

Sulfide

Sulfide

Sulfotepp

2,4,5-T

2,3,7,8-TCDD

2,3,7,8-TCDF

1,2,4,5-Tetrachlorobenzene

Tetrachlorodibenzo-p-dioxins

Tetrachlorodibenzo-p-furans

1,1,1,2-Tetrachloroethane

$1,1,2,2-$ Tetrachioroethane

Tetrachloroethyiene

2,3,4,6-Tetrachlorophenol

Thallium, total recoverable

Thionazin

Tin, total recoverable

Toluene

o-Toluidine

Toxaphene

2,4,5-TP (Silvex)

1,1,1-Trichloroethane

1,1,2-Trichloroethane

Trichloroethylene

Trichlorofluoromethane

2,4,5-Trichloropheno

2,4,6-Trichlorophenol

1,2,3-Trichioropropane

$0,0,0$-Triethyl phosphorothioate

1,3,5-Trinitrobenzene

Vanadium, total recoverable

Vínyl acetate

Xylenes

Zinc, total recoverable

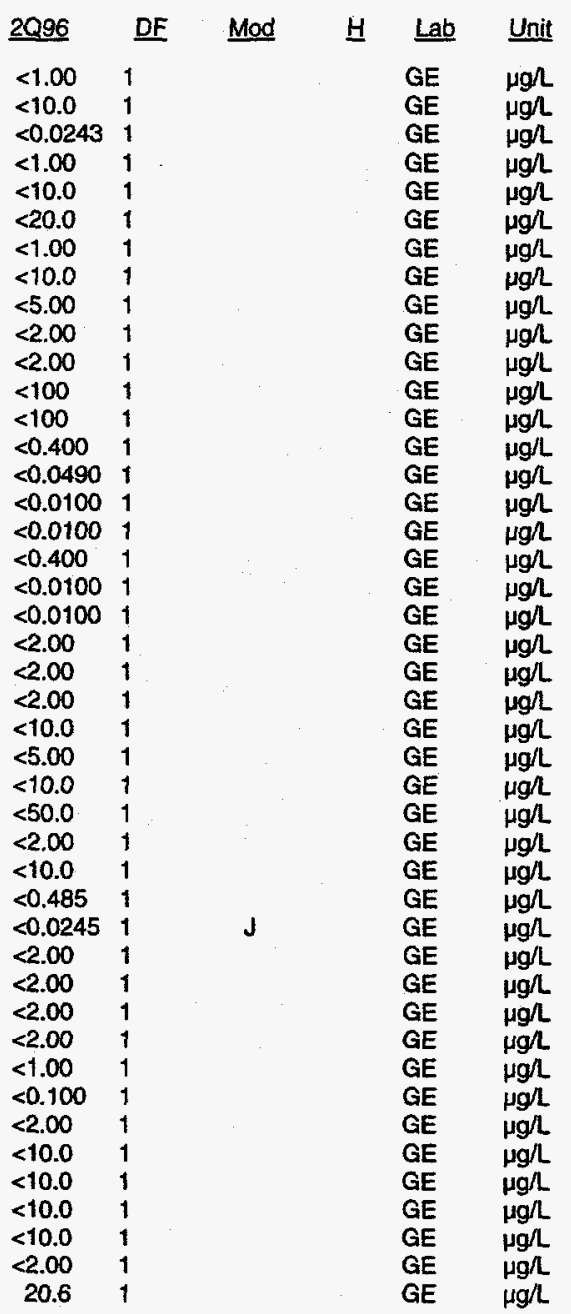

Note: Modifiers are listed and detailed in Appendix D, Key to Reading the Tables.

- = exceeded holding time. 
WELL LFW 74D

$\begin{array}{lc}\text { SRS Coord. } & \text { Lat/Longitude } \\ \text { N85828.1 } & 33.289172^{\circ} \mathrm{N} \\ \text { E45098.0 } & 81.714771^{\circ} \mathrm{W} \\ \text { SAMPLE DATE } & \text { O4/22/96 }\end{array}$

Screen Zone Elevation

$167.7-152.7 \mathrm{ft} \mathrm{msl}$
Top of Casing

$213.9 \mathrm{ft} \mathrm{mst}$
Casing

4" PVC
Pump

S
Screen Zone

Upper Steed Pond

\section{FIELD DATA}

Parameter

Water elevation

$\mathrm{pH}$

Sp. conductance

Water temperature

Alkalinity as $\mathrm{CaCO}_{3}$

Turbidity

Volume purged

Sampling code

ANALYTICAL DATA
162.8

4.8

18

25.0

1

3.0
Unit

ft msl

$\mathrm{pH}$

$\mu \mathrm{S} / \mathrm{cm}$

${ }^{\circ} \mathrm{C}$

mg/L

well vol
Parameter

Acenaphthylene

Acetone

Acetonitrile (Methyl cyanide)

Acetophenone

2-Acetylaminofluorene

Acrolein

Acrylonitrile

Aldrin

Allyl chloride

4-Aminobiphenyl

Aniline

Anthracene

Antimony, total recoverable

Antimony, total recoverable

Aramite

Arsenic, total recoverable

Arsenic, total recoverable

Barium, total recoverable

Barium, total recoverable

Benzene

alpha-Benzene hexachloride

beta-Benzene hexachloride

delta-Benzene hexachloride

Benzo(a)anthracene

Benzo(b)fluoranthene

Benzo(k)fluoranthene

Benzo(g,h,i)perylene

Benzo(a)pyrene

Benzyl alcohol

Beryllium, total recoverable

Beryllium, total recoverable

Bis(2-chloroethoxy) methane

Bis(2-chloroethyl) ether

Bis(2-chloroisopropyl) ether

Bis(2-ethylhexyl) phthalate

Bromodichloromethane

Bromoform

Bromomethane

4-Bromophenyl phenyl ether

Butylbenzyl phthalate

2-sec-Butyl-4,6-dinitrophenol

Cadmium, total recoverable

Cadmium, total recoverable

Carbon disulfide

Carbon tetrachloride

Chlordane

4-Chloroaniline

Chlorobenzene

Chlorobenzilate

Chloroethane

Chloroethene (Vinyl chloride)

Chloroform

\begin{tabular}{|c|c|c|c|c|}
\hline 2096 & $\underline{\mathrm{DF}}$ & Mod & 브 & Lat \\
\hline$<1.00$ & 1 & & & GE \\
\hline$<10.0$ & 1 & & & $\overrightarrow{\mathrm{GE}}$ \\
\hline$<10.0$ & 1 & & & GE \\
\hline$<1.00$ & 1 & & & GE \\
\hline$<10.0$ & 1 & & & $\mathrm{GE}$ \\
\hline$<20.0$ & 1 & & & GE \\
\hline$<50.0$ & 1 & & & GE \\
\hline$<0.0124$ & 1 & & & GE \\
\hline$<10.0$ & 1 & & & GE \\
\hline$<10.0$ & 1 & & & GE \\
\hline$<1.00$ & 1 & & & GE \\
\hline$<1.00$ & 1 & & & $\overline{G E}$ \\
\hline 1.83 & 1 & JE & & $\mathrm{GE}$ \\
\hline 1.87 & 1 & JE & & GE \\
\hline$<10.0$ & 1 & & & GE \\
\hline$<5.00$ & 1 & & & $\mathrm{GE}$ \\
\hline$<5.00$ & 1 & & & GE \\
\hline 3.05 & 1 & $V$ & & GE \\
\hline 2.92 & 1 & JEV & & $\mathrm{GE}$ \\
\hline$<2.00$ & 1 & & & GE \\
\hline$<0.0124$ & 1 & & & GE \\
\hline 0.0331 & 1 & & & $\overline{\mathrm{GE}}$ \\
\hline 0.015 & 1 & JE & & $\overline{\mathrm{GE}}$ \\
\hline$<0.100$ & 1 & & & $\overline{\mathrm{GE}}$ \\
\hline$<0.100$ & 1 & & & $\overrightarrow{\mathrm{GE}}$ \\
\hline$<0.100$ & 1 & & & $\overline{G E}$ \\
\hline$<1.00$ & 1 & & & $\overline{\mathrm{GE}}$ \\
\hline$<0.100$ & $i$ & & & $\mathrm{GE}$ \\
\hline$<1.00$ & $i$ & & & GE \\
\hline$<0.355$ & 1 & v & & GE \\
\hline$<0.381$ & 1 & V & & GE \\
\hline$<1.00$ & 1 & & & $\mathrm{GE}$ \\
\hline$<1.00$ & 1 & & & $\mathrm{GE}$ \\
\hline$<20.0$ & 1 & & & GE \\
\hline$<1.00$ & 1 & & & $\overline{\mathrm{GE}}$ \\
\hline$<2.00$ & 1 & & & $\overline{\mathrm{GE}}$ \\
\hline$<2.00$ & 1 & & & $\overline{\mathrm{GE}}$ \\
\hline$<2.00$ & 1 & & & $\mathrm{GE}$ \\
\hline$<1.00$ & 1 & & & GE \\
\hline$<1.00$ & 1 & & & $\overline{G E}$ \\
\hline$<10.0$ & 1 & & & $\overline{\mathrm{GE}}$ \\
\hline$<2.00$ & 1 & & & $\overline{G E}$ \\
\hline$<2.00$ & 1 & & & GE \\
\hline$<5.00$ & $i$ & & & $\mathrm{GE}$ \\
\hline$<2.00$ & 1 & & & GE \\
\hline$<0.124$ & 1 & & & $\overline{G E}$ \\
\hline$<1.00$ & 1 & & & $\mathrm{GE}$ \\
\hline$<2.00$ & 1 & & & GE \\
\hline$<10.0$ & 1 & & & GE \\
\hline$<2.00$ & 1 & & & $\mathrm{GE}$ \\
\hline$<2.00$ & 1 & & & $\mathrm{GE}$ \\
\hline$<2.00$ & 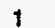 & & & $\mathrm{GE}$ \\
\hline
\end{tabular}

Note: Modifiers are listed and detailed in Appendix D, Key to Reading the Tables.

- exceeded hoiding time. 
Well LFW 74D (cont.)

Parameter

\begin{tabular}{|c|c|c|c|c|}
\hline 2096 & $\underline{D F}$ & Mod & $\underline{H}$ & Lab \\
\hline$<2.00$ & 1 & & & GE \\
\hline$<1.00$ & 1 & & & \\
\hline$<1.00$ & 1 & & & GE \\
\hline$<2.00$ & 1 & & & GE \\
\hline$<4.00$ & 1 & & & $\mathrm{GE}$ \\
\hline$<4.00$ & 1 & & & GE \\
\hline$<0.100$ & 1 & & & GE \\
\hline$<0.399$ & 1 & V & & GE \\
\hline$<0.321$ & 1 & v & & GE \\
\hline$<4.00$ & 1 & & & GE \\
\hline 0.874 & 1 & JE & & GE \\
\hline$<10.0$ & 1 & & & GE \\
\hline$<1.00$ & 1 & & & GE \\
\hline$<10.0$ & 1 & & & GE \\
\hline$<0.0495$ & 1 & & & GE \\
\hline$<0.0248$ & 1 & & & GE \\
\hline$<0.0495$ & 1 & & & GE \\
\hline$<10.0$ & 1 & & & GE \\
\hline$<0.100$ & 1 & & & GE \\
\hline$<1.00$ & 1 & & & GE \\
\hline$<2.00$ & 1 & & & GE \\
\hline$<2.00$ & 1 & & & GE \\
\hline$<2.00$ & 1 & & & GE \\
\hline$<2.00$ & 1 & & & GE \\
\hline 1.1 & 1 & & & GE \\
\hline$<1.00$ & 1 & & & GE \\
\hline$<1.00$ & 1 & & & GE \\
\hline$<1.00$ & 1 & & & GE \\
\hline$<2.00$ & 1 & & & GE \\
\hline$<2.00$ & 1 & & & GE \\
\hline$<2.00$ & 1 & & & GE \\
\hline$<2.00$ & 1 & & & GE \\
\hline$<2.00$ & 1 & & & GE \\
\hline$<2.00$ & 1 & & & GE \\
\hline 2.56 & 1 & JE & & GE \\
\hline$<1.00$ & 1 & & & GE \\
\hline$<10.0$ & 1 & & & GE \\
\hline$<0.0495$ & $i$ & & & GE \\
\hline$<2.00$ & 1 & & & GE \\
\hline$<2.00$ & 1 & & & GE \\
\hline$<2.00$ & 1 & & & GE \\
\hline$<0.0248$ & 1 & & & GE \\
\hline$<1.00$ & 1 & & & GE \\
\hline$<1.00$ & 1 & & & GE \\
\hline$<1.00$ & 1 & & & GE \\
\hline$<1.00$ & 1 & & & GE \\
\hline$<10.0$ & 1 & & & GE \\
\hline$<10.0$ & 1 & & & GE \\
\hline$<10.0$ & 1 & & & GE \\
\hline$<10.0$ & 1 & & & GE \\
\hline$<10.0$ & 1 & & & GE \\
\hline$<10.0$ & 1 & & & GE \\
\hline$<0.100$ & 1 & & & GE \\
\hline$<1.00$ & 1 & & & GE \\
\hline$<10.0$ & 1 & & & GE \\
\hline$<10.0$ & 1 & & & GE \\
\hline$<1.00$ & 1 & & & GE \\
\hline$<0.0495$ & 1 & & & GE \\
\hline$<0.0248$ & 1 & & & GE \\
\hline$<0.0495$ & 1 & & & GE \\
\hline$<0.0248$ & 1 & & & GE \\
\hline$<0.0619$ & 1 & & & GE \\
\hline$<10.0$ & 1 & & & GE \\
\hline$<10.0$ & 1 & & & GE \\
\hline$<2.00$ & 1 & & & GE \\
\hline$<10.0$ & 1 & & & GE \\
\hline$<1.00$ & 1 & & & GE \\
\hline$<1.00$ & 1 & & & GE \\
\hline$<0.0248$ & 1 & & & GE \\
\hline$<0.0248$ & 1 & & & $G E$ \\
\hline$<0.0100$ & 1 & & & GE \\
\hline$<0.0100$ & 1 & & & GE \\
\hline
\end{tabular}

Note: Modifiers are listed and detailed in Appendix D, Key to Reading the Tables.

- = exceeded holding time. 
Well LFW 74D (cont.)

\section{Parameter}

Hexachlorobenzene

Hexachlorobutadiene

Hexachlorocyclopentadiene

Hexachlorodibenzo-p-dioxins

Hexachlorodibenzo-p-turans

Hexachloroethane

Hexachlorophene

Hexachloropropene

2-Hexanone

1,2,3,4,6,7,8-HPCDD

$1,2,3,4,6,7,8$-HPCDF

$1,2,3,4,7,8-\mathrm{HXCDD}$

$1,2,3,4,7,8-\mathrm{HXCDF}$

Indeno(1,2,3-c,d)pyrene

lodomethane (Methyl iodide)

Isobutyl alcohol

Isodrin

Isophorone

Isosafrole

Kepone

Lead, total recoverable

Lead, total recoverable

Lindane

Manganese, total recoverable

Mercury, total recoverable

Methapyriene

Methoxychlor

2-Methyl-4,6-dinitropheno

Methyl ethyl ketone

Methyl isobutyl ketone

Methyl methacrylate

Methyi methanesulfonate

3-Methylcholanthrene

2-Methylnaphthalene

Naphthalene

1,4-Naphthoquinone

1-Naphthylamine

2-Naphthylamine

Nickel, total recoverable

Nickel, total recoverable

$m$-Nitroaniline

o-Nitroaniline

p-Nitroaniline

Nitrobenzene

2-Nitropheno

4-Nitrophenol

4-Nitroquinoline-1-oxide

N-Nitrosodi-n-butylamine

$\mathrm{N}$-Nitrosodiethylamine

$\mathrm{N}$-Nitrosodimethylamine

$\mathrm{N}$-Nitrosodiphenylamine

$\mathrm{N}$-Nitrosomethylethylamine

$\mathrm{N}$-Nitrosomorpholine

N-Nitrosopiperidine

$\mathrm{N}$-Nitrosopyrrolidine

5-Nitro-0-toluidine

Octachlorodibenzo-p-dioxin

Octachlorodibenzo-p-furan

Parathion

Parathion methyl

PCB 1016

PCB 1016

PCB 1221

PCB 1221

PCB 1232

PCB 1232

PCB 1242

PCB 1242

PCB 1248

PCB 1248

PCB 1254

PCB 1254
Methacrylonitrile

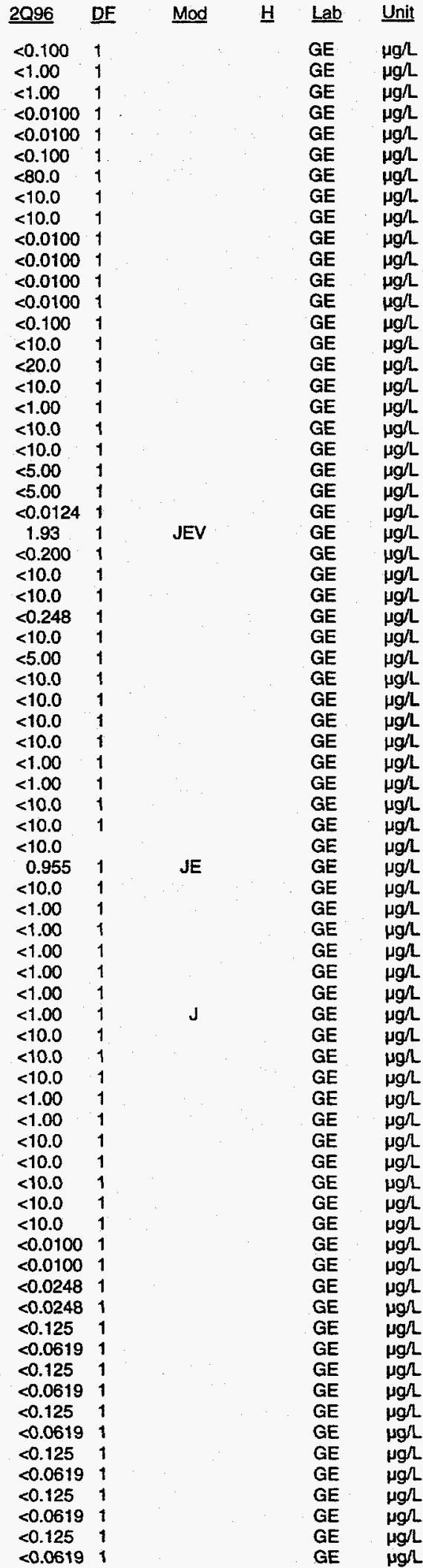

Note: Modifiers are listed and detailed in Appendix D, Key to Reading the Tables. - = exceeded holding time. $\mu g / 2$

$\mu g$ /

wall

$\mu g / L$

ugh

igh

gh

$\mu \mathrm{g} / \mathrm{L}$

$\mu g h$

$\mu g / L$

gl

igh

$\mu g / L$

$\mu g / L$

$\mu g / L$

g'L

gh/

$\mu g / L$

gh

$\mu g / L$

$\mu g / L$

$\mu g h$

$\mu g$ h

$\mu g / L$

$\mu g / 2$

$\mu g / 2$

gh.

$\mu g / 2$

$\mu g$ L

gh

$\mu \mathrm{g} / \mathrm{L}$

g/

gh

$\mu g$ L

ugh

$\mu g / 2$

gr

$\mu g /$

$\mu g / 2$

g $\Omega$

I

gl

$\mu g / 2$

ugl

$\mu g / L$

on 
Well LFW 74D (cont.)

Parameter

PCB 1260

PCB 1260

$1,2,3,7,8-P C D D$

1,2,3,7,8-PCDF

Pentachlorobenzene

Pentachlorodibenzo-p-dioxins

Pentachlorodibenzo-p-furans

Pentachloroethane

Pentachloronitrobenzene

Phenacetin

Phenanthrene

p-Phenylenediamine

Phorate

2-Picoline

Pronamid

Propionitrile

Pyridine

Safrole

Selenium, total recoverable

Selenium, total recoverable

Silver, total recoverable

Silver, total recoverable

Styrene

Sulfide

Sulfotepp

2,4,5-T

2,3,7,8-TCDD

$2,3,7,8$-TCDF

1,2,4,5-Tetrachlorobenzene

Tetrachlorodibenzo-p-dioxins

Tetrachlorodibenzo-p-furans

1,1,1,2-Tetrachloroethane

$1,1,2,2$-Tetrachloroethane

Tetrachloroethylene

2,3,4,6-Tetrachlorophenol

Thallium, total recoverable

Thallium, total recoverable

Thionazin

Tin, total recoverable

Tin, total recoverable

Toluene

o-Toluidine

Toxaphene

2,4,5-TP (Silvex)

1,1,1-Trichloroethane

1,1,2-Trichloroethane

Trichloroethylene

Trichlorofluoromethane

2,4,5-Trichlorophenol

2,4,6-Trichlorophenol

1,2,3-Trichloropropane

\begin{tabular}{|c|c|c|c|c|}
\hline$\underline{2096}$ & DF & Mod & $\underline{H}$ & Lab \\
\hline$<0.0619$ & 1 & & & GE \\
\hline$<0.125$ & 1 & $J$ & & $\mathrm{GE}$ \\
\hline$<0.0100$ & 1 & & & GE \\
\hline$<0.0100$ & 1 & & & $\mathrm{GE}$ \\
\hline$<10.0$ & 1 & & & GE \\
\hline$<0.0100$ & 1 & & & GE \\
\hline$<0.0100$ & 1 & & & GE \\
\hline$<10.0$ & 1 & & & GE \\
\hline$<10.0$ & 1 & & & GE \\
\hline$<10.0$ & 1 & & & GE \\
\hline$<1.00$ & 1 & & & GE \\
\hline$<10.0$ & 1 & & & GE \\
\hline$<0.0248$ & 1 & & & GE \\
\hline$<1.00$ & 1 & & & GE \\
\hline$<10.0$ & 1 & & & GE \\
\hline$<20.0$ & 1 & & & GE \\
\hline$<1.00$ & 1 & & & $\mathrm{GE}$ \\
\hline$<10.0$ & 1 & & & $G E$ \\
\hline$<5.00$ & $i$ & & & GE \\
\hline$<5.00$ & 1 & & & $\overline{G E}$ \\
\hline$<2.00$ & 1 & & & GE \\
\hline$<2.00$ & 1 & & & GE \\
\hline$<2.00$ & 1 & & & $\mathrm{GE}$ \\
\hline$<100$ & 1 & & & GE \\
\hline$<0.400$ & 1 & & & GE \\
\hline$<0.0495$ & $i$ & & & $\overline{G E}$ \\
\hline$<0.0100$ & 1 & & & $\mathrm{GE}$ \\
\hline$<0.0100$ & 1 & & & GE \\
\hline$<0.400$ & 1 & & & $\mathrm{GE}$ \\
\hline$<0.0100$ & 1 & & & GE \\
\hline$<0.0100$ & 1 & & & $\overrightarrow{\mathrm{GE}}$ \\
\hline$<2.00$ & 1 & & & $\mathrm{GE}$ \\
\hline$<2.00$ & 1 & & & GE \\
\hline$<2.00$ & 1 & & & GE \\
\hline$<10.0$ & 1 & & & $\mathrm{GE}$ \\
\hline$<5.00$ & 1 & & & GE \\
\hline$<5.00$ & 1 & & & GE \\
\hline$<10.0$ & 1 & & & GE \\
\hline$<50.0$ & 1 & & & $\mathrm{GE}$ \\
\hline$<50.0$ & 1 & & & GE \\
\hline$<2.00$ & 1 & & & GE \\
\hline$<10.0$ & 1 & & & $\mathrm{GE}$ \\
\hline$<0.495$ & 1 & & & GE \\
\hline$<0.0248$ & 1 & J & & GE \\
\hline$<2.00$ & $i$ & & & $\overline{G E}$ \\
\hline$<2.00$ & 1 & & & GE \\
\hline$<2.00$ & $i$ & & & GE \\
\hline$<2.00$ & 1 & & & $\mathrm{GE}$ \\
\hline$<1.00$ & 1 & & & GE \\
\hline$<0.100$ & 1 & & & $\mathrm{GE}$ \\
\hline$<2.00$ & 1 & & & GE \\
\hline$<10.0$ & 1 & & & GE \\
\hline$<10.0$ & 1 & & & GE \\
\hline$<0.393$ & 1 & v & & $\mathrm{GE}$ \\
\hline$<10.0$ & 1 & & & $G E$ \\
\hline$<10.0$ & 1 & & & $\mathrm{GE}$ \\
\hline$<2.00$ & 1 & & & $\mathrm{GE}$ \\
\hline 6.53 & 1 & & & GE \\
\hline 6.2 & 1 & & & $\mathrm{GE}$ \\
\hline
\end{tabular}

-Triethyl phosphorothioat

1,3,5-Trinitrobenzene

Vanadium, total recoverable

Vanadium, total recoverable

Vinyl acetate

Xylenes

Zinc, total recoverable

Zinc, total recoverable

Note: Modifiers are listed and detailed in Appendix D, Key to Reading the Tables.

- = exceeded holding time. 
THIS PAGE LEFT BLANK INTENTIONALLY. 